\title{
In search of balance
}

Citation for published version (APA):

Merkx, A. C. M. J. (2017). In search of balance: promoting healthy gestational weight gain. [Doctoral Thesis, Maastricht University]. Maastricht University. https://doi.org/10.26481/dis.20170519am

Document status and date:

Published: 01/01/2017

DOI:

10.26481/dis.20170519am

Document Version:

Publisher's PDF, also known as Version of record

\section{Please check the document version of this publication:}

- A submitted manuscript is the version of the article upon submission and before peer-review. There can be important differences between the submitted version and the official published version of record.

People interested in the research are advised to contact the author for the final version of the publication, or visit the DOI to the publisher's website.

- The final author version and the galley proof are versions of the publication after peer review.

- The final published version features the final layout of the paper including the volume, issue and page numbers.

Link to publication

\footnotetext{
General rights rights.

- You may freely distribute the URL identifying the publication in the public portal. please follow below link for the End User Agreement:

www.umlib.nl/taverne-license

Take down policy

If you believe that this document breaches copyright please contact us at:

repository@maastrichtuniversity.nl

providing details and we will investigate your claim.
}

Copyright and moral rights for the publications made accessible in the public portal are retained by the authors and/or other copyright owners and it is a condition of accessing publications that users recognise and abide by the legal requirements associated with these

- Users may download and print one copy of any publication from the public portal for the purpose of private study or research.

- You may not further distribute the material or use it for any profit-making activity or commercial gain

If the publication is distributed under the terms of Article $25 \mathrm{fa}$ of the Dutch Copyright Act, indicated by the "Taverne" license above, 


\section{IN SEARCH OF BALANCE}

\section{Promoting healthy gestational weight gain}




\title{
opgedragen aan
}

\section{de Grote Chaotische Orde}

\author{
die het Leven is
}

Coverdesign and print: Creatieve communicatie, Leon Hoenen

Foto cover (kunstwerk EA) gemaakt door Irma Gelsing

Hulp bij redactie: Jacqueline Schoonheim, Frans Merkx, Tim Walpot

ISBN: 978-90-804229-0-2

\section{Copyright (C) 2017 Astrid Merkx}

No part of this thesis may be reproduced, stored in a retrieval system or transmitted in any form or by any means without permission from the author or, when appropriate, from the publisher of the publications. 


\title{
IN SEARCH OF BALANCE
}

\section{Promoting healthy gestational weight gain}

\author{
PROEFSCHRIFT
}

ter verkrijging van de graad van doctor

aan de Universiteit van Maastricht

op gezag van de Rector Magnificus Prof. dr. Rianne M. Letschert

volgens het besluit van het College der Decanen,

in het openbaar te verdedigen

op vrijdag 19 mei 2017 om 10.00 uur

door

Astrid Cornelia Maria Joseph Merkx 


\section{Promotor}

Prof. dr. Raymond G. de Vries (University of Michigan)

\section{Copromotores}

Dr. Marlein Ausems

Dr. Marianne J. Nieuwenhuijze

\section{Beoordelingscommissie}

Prof. Dr. Onno van Schayck (voorzitter)

Prof. Dr. Katrien Beeckman (Universitair Ziekenhuis Brussel)

Dr. Jascha M. de Nooijer

Prof. Dr. Eric A.P. Steegers (Erasmus MC)

Subsidieverstrekker: Regional Attention and Action for Knowledge (RAAK), Stichting Innovatie Alliantie (SIA) (RAAK, ref. PRO 2-014)

Het verschijnen van dit proefschrift werd mede mogelijk gemaakt door de steun van de Koninklijke Nederlandse Organisatie van Verloskundigen (KNOV). 


\section{Contents}

$\begin{array}{lll}\text { Chapter } 1 \text { General introduction } & 7\end{array}$

Chapter 2 Interventions aiming to achieve a healthy gestational weight gain: A systematic review / meta-analysis

Chapter 3 Weight gain in healthy pregnant women in relation to prepregnancy body mass index, diet and physical activity

Chapter 4 How and why do healthy pregnant women change their physical activity in pregnancy?

Chapter 5 Dutch Midwives' Behaviour and Determinants in Promoting Healthy Gestational Weight Gain, Phase 1: A Qualitative Approach

Chapter 6 Dutch Midwives' Behaviour and Determinants in Promoting Healthy Gestational Weight Gain, Phase 2: A Quantitative Approach

Chapter 7 “Come On!” Using Intervention Mapping to help healthy pregnant women achieve healthy weight gain.

Chapter 8 "Come on!" Pilot-study of an intervention, targeting on healthy gestational weight gain.

Chapter 9 Summary and general discussion

Large tables and figure $\mathbf{2 . 2}$ 

Chapter 1

\section{General Introduction}




\subsection{General introduction}

Healthy gestational weight gain (GWG) is associated with better pregnancy outcomes and with improved health in the later lives of women and babies. In this thesis we describe the process of developing an intervention to help pregnant women reach a healthy GWG. The need for this intervention was derived from discussions with midwives, working in primary care in the Netherlands. In this introduction, I describe the background of the larger project "Promoting Health Pregnancy", of which this study is a part (1.2), the problem of unhealthy GWG (1.3-1.6) and offer a brief introduction to the theoretical framework of the study and to the subsequent chapters (1.7-1.9).

\subsection{Background of the project}

The project "Promoting Healthy Pregnancy" was developed in response to the concerns expressed by midwives in primary care practices regarding the health of mothers and babies. Researchers at the Research Centre for Midwifery Science, Maastricht-Zuyd University (Academie Verloskunde Maastricht - AVM) discovered that midwives were having particular difficulties in responding to the needs of two kinds of women: pregnant women struggling with maternal distress ${ }^{1}$ and pregnant women who were overweight or obese. The focus in this thesis is therefor on the latter problem. In studying the problems associated with GWG, we learned that GWG was an important predictor for overweight and obesity in the long term, ${ }^{2-5}$ underscoring the need for every woman to reach a healthy GWG. ${ }^{6,7}$

The goal of my work in the "Promoting Healthy Pregnancy" project was to provide midwives with evidence based tools to help women to develop a healthy GWG. To achieve this goal, we used a bottom-up approach, involving experts in the field of weight management, practicing midwives, and pregnant women to design an evidence-informed intervention. As part of this approach we established a Consortium, including community midwives, a dietician, a physiotherapist, a psychologist working with pregnant women and young mothers, a member of the Royal Dutch Organisation of Midwives (Koninklijke Nederlandse Organisatie van Verloskundigen - KNOV) and experts on health promotion. The Consortium guided and supported the research team in developing the intervention. During the design process, pregnant women and young mothers provided information about relevant GWG aspects and reviewed and commented on drafts of the intervention components.

The project "Promoting Healthy Pregnancy" was funded by Regional Attention and Action for Knowledge (RAAK), registered as RAAK PRO 2-014. RAAK is managed by the National Taskforce Practice-oriented Research (Nationaal Regie orgaan praktijkgericht onderzoek -SIA), which is funded by the Ministry of Education, Culture and Science (Ministerie van Onderwijs en Wetenschappen - OCW). 


\subsection{Healthy gestational weight gain: definition and recommendations}

When a woman gets pregnant, it is normal that she gains weight. Thirty years ago, when obesity was found mostly in people who were wealthy or had a thyroid dysfunction, the Dutch textbook for physiology of childbirth taught that $12 \mathrm{~kg}$ was an average GWG. ${ }^{8}$ This book also stated that at term, the mean bodyweight of a baby is $3.4 \mathrm{~kg}$, amniotic fluid weighs around $0.8 \mathrm{~kg}$ and the weight of the placenta and membranes is approximately $0.6 \mathrm{~kg} .{ }^{8} \mathrm{The}$ text went on to note that the physical system of the woman will adapt to the growing foetus by increasing the weight of organs that need to adapt to demands of the pregnant body: for example, the growing uterus and breasts. This leads also to an increased vascularisation and elevated blood volume. Weight gain involved in this process was estimated to be $5.0 \mathrm{~kg} .{ }^{8}$ Finally, the authors of the text pointed out that a woman's body will prepare for the time after birth when she is expected to breastfeed her baby. It is therefore normal that she stores $2.2 \mathrm{~kg}$ fat during pregnancy that can be used in the time after birth ${ }^{8}$ when she is less able to fulfil her evolutionary task of "seeking for food". In total then, healthy GWG was estimated to be around $12 \mathrm{~kg}$. In recent research, the mean of $12 \mathrm{~kg} \mathrm{GWG}$ for healthy women has been confirmed to be a healthy GWG. ${ }^{9}$ The old midwifery textbook did not recommend adjusting GWG to pre-pregnancy weight - e.g., women who were "thin" at the start of pregnancy were not instructed to gain more weight than normal weight women. However, the author, who attended midwifery school 25 years ago, was told that in practice she should use such recommendation. She was also instructed that "larger" women, who already had some fat storage, should gain less weight during their pregnancy.

It may seem obvious that efforts to limit GWG require a means to measure GWG. However, for several reasons, it is hard to provide a clear definition of GWG. Not every woman weighs herself before she gets pregnant, women tend to fluctuate in their bodyweight between weekend and weekdays, ${ }^{10}$ and dietary constraints or weight gain goals influence the measurement of weight. ${ }^{11,12}$ Interestingly, there is no agreement about when the baseline measurement for GWG should occur. Some women lose weight in early pregnancy, as a result of nausea or vomiting, and some gain weight in that time. ${ }^{6,13}$ Therefore, counting from the first antenatal visit (gestational age of 6 to 10 weeks) is not a valid beginning point of GWG. Additionally, it is also difficult to state a clear end point for measuring GWG. Different studies use different ending points; at 36 weeks gestation, at the last prenatal consultation or at the onset of labour. ${ }^{6}$ One solution to this problem of measurement is to estimate GWG using weight gain per week, starting at a time point in pregnancy where most women are not sick anymore. This approach allows comparison between studies, but does not solve the issue of weight changes (gain or loss) in the first trimester. Because weight changes in this period are important, some researchers choose to work with self-reported pre-pregnancy weight. ${ }^{6}$ To date, an official Dutch guideline on GWG does not exist. In 1990, guidelines for GWG were published by the Institute of Medicine (IOM) in the United States of America (USA) $;^{14}$ these guidelines were updated in 2009. ${ }^{6}$ The IOM recommends a total GWG based on the Body 
Mass Index (BMI) before pregnancy (hereafter BMI will refer to pre-pregnancy BMI). The recommended healthy GWG is the same in the first thirteen weeks for all BMI groups (underweight, normal weight, overweight and obese); from 0.5 to 2.0 kilogram. In the second and third trimesters of pregnancy the guidelines recommend a maximum and minimum rate of GWG per week, depending on the BMI. ${ }^{6}$ The two important differences in the 2009 update were: (1) the classification of the BMI groups in a manner similar to the definitions used by the World Health Organisation (WHO) and (2) a maximum cut-off point for GWG in obese women. Evidence to underpin the cut-off points was based on limited research, and the authors relied mostly on clinical experiences and expert consensus. ${ }^{6}$

Table 1.1 Classification of the Body Mass Index (BMI) in groups (as used by World Health Organization (WHO), the 1990 Institute of Medicine (IOM) guidelines and the 2009 IOMguidelines) and recommendations for gestational weight gain (GWG) for different BMI groups according to the 2009 IOM-guidelines.

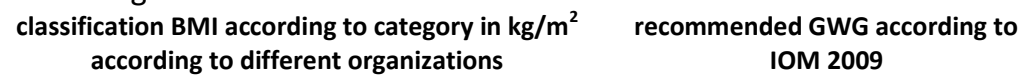

\begin{tabular}{|c|c|c|c|c|c|}
\hline & WHO & $\begin{array}{l}1990 \text { IOM- } \\
\text { guidelines }\end{array}$ & $\begin{array}{l}2009 \text { IOM- } \\
\text { guidelines }\end{array}$ & $\begin{array}{l}\text { Total GWG } \\
(\mathrm{kg})\end{array}$ & $\begin{array}{l}\text { GWG rate per } \\
\text { week }^{\mathrm{a}}(\mathrm{kg} / \text { week })\end{array}$ \\
\hline underweight & $<18.5$ & $<19.8$ & $<18.5$ & $12.5-18$ & 0.51 \\
\hline normal weight & $18.5-24.9$ & $19.8-26.0$ & $18.5-24.9$ & $11.5-16$ & 0.42 \\
\hline overweight & $25.0-29.9$ & $26.0-29.0$ & $25.0-29.9$ & $7-11.5$ & 0.28 \\
\hline obese class I & $30.0-34.9$ & $\geq 29.0$ & $\geq 30.0$ & $5-9$ & 0.22 \\
\hline obese class II & $35.0-39.9$ & & & & \\
\hline obese class III & $\geq 40.0$ & & & & \\
\hline
\end{tabular}

Abbreviations: BMI, Body Mass Index; IOM, Institute of Medicine; GWG, gestational weight gain; kg, kilograms; WHO, World Health Organisation

${ }^{\mathrm{a}}$ in the second and third trimester of pregnancy. In the first trimester recommended weight gain is 0.5 to $2.0 \mathrm{~kg}$ for all BMI groups

We chose to use the IOM-guidelines as the reference in our project, because: 1 ) these guidelines were the best available guidelines at the start of the project and 2) since nearly all GWG related research used the IOM-guidelines. ${ }^{6}$ Table 1.1 presents the classification of BMI groups according to the WHO, the 1990 IOM-guidelines and the 2009 IOM-guidelines along with the recommendations of GWG for different BMI groups. In this thesis, when we refer to "IOM-guidelines", we mean the IOM-guidelines dated 2009. We use the words "excessive" or "too high" GWG for GWG above the IOM-guidelines and we use "inadequate" or "too low" GWG for GWG beneath the IOM-guidelines. Furthermore we use "healthy GWG" and "adequate GWG" for GWG within the IOM-guidelines and "unhealthy GWG" for GWG above or below the IOM-guidelines. Finally, the word "overweight" is used for overweight as categorized according to the WHO. In the context of "too high bodyweight" we use the term "over-weight". 


\subsection{The importance of gaining weight within the guidelines}

When we began our research, data from the Euro-Peristat Network - an information system intended to help policymakers make decisions about the health care of pregnant women and newborns - created concern about perinatal death in the Netherlands. At the start of our study, Dutch perinatal death numbers were reported to be one of the highest in Europe. ${ }^{15,16}$ Although the cross country comparisons proved to be problematic, the report focussed attention on strategies to improve the health of mothers and babies. One of the main contributors for perinatal death is preterm birth and low birthweight/small for gestational age (sga) babies. ${ }^{17}$ Too high GWG and too low GWG contribute directly and indirectly to complications in pregnancy and birth, including preterm birth and sga, which in turn contribute to higher perinatal death rates. ${ }^{18,19}$ Table 1.2 provides an overview of reviews reporting on negative outcomes associated with GWG. GWG above the guidelines is associated with over-weight in the long term for mothers and babies. Compared with women who gain weight within the guidelines, women who exceed the IOM-guidelines have a mean increase in post-partum weight retention that varies between $2.45 \mathrm{~kg}$ (95\% Cl 1.95-2.95) in the first postpartum year, to $4.72 \mathrm{~kg}(95 \% \mathrm{Cl}: 2.94,6.50 \mathrm{~kg}) 15$ years postpartum. ${ }^{20}$ The offspring of women with high GWG have higher birthweight, higher weight in childhood and adolescence, and are more often over-weight as an adult than the offspring of women who have an adequate GWG. ${ }^{21-26}$ Excessive GWG is associated with a higher likelihood of gestational diabetes mellitus (GDM) and foetal hyperglycaemia, ${ }^{22}$ Caesarean section, ${ }^{19}$ preterm birth, ${ }^{19,27}$ and negative psychological outcomes. ${ }^{28,29}$ Inadequate GWG is associated with a higher likelihood of low birthweight ${ }^{18,19,30}$ and preterm birth, ${ }^{18,19,30}$ and a lower likelihood to initiate breastfeeding. ${ }^{19}$ A study of interventions to increase the likelihood of healthy GWG revealed that interventions that lowered GWG had no effect on pre-eclampsia, GDM and inductions, ${ }^{31}$ while another review of intervention studies showed a significant impact on hypertension. ${ }^{32}$

Single cohort studies, not included in the previously mentioned reviews found additional associations between excessive GWG and higher likelihood of post-partum haemorrhage, ${ }^{33}$ instrumental birth, ${ }^{34}$ hypertension, ${ }^{35}$ augmentation rate, ${ }^{35}$ Caesarean section, ${ }^{35}$ low Free Thyroxine 4 and high Thyroid Stimulating Hormone levels, ${ }^{36}$ neonatal asphyxia, ${ }^{37}$ respiratory distress syndrome, respiratory disease and longer postnatal length of hospital stay ${ }^{38}$ and cardio-metabolic risk factors in 20 year old offspring. ${ }^{39}$ Excessive GWG also complicates the subsequent pregnancy: when the BMI category of women increased with 1 to 2 units between pregnancies, women had an increased risk of hypertension in the next pregnancy. ${ }^{40}$

Table 1.2 Reviews on effects of Gestational Weight Gain (GWG) on pregnancy outcomes

\begin{tabular}{llll}
\hline Study & Design & Sample & Findings \\
\hline $\mathrm{O}^{\prime}$ Brien, & Review of & 5 studies, $\mathrm{n}=714 \mathrm{NW}$, & Interventions lead to less GWG: $-1.25 \mathrm{~kg}(95 \%$ \\
$2016^{32}$ & interventions & primary and secondary & $\mathrm{Cl} 2.39$ to -0.11$)$ and less $>10 \mathrm{M} \mathrm{RR} \mathrm{0.66} \mathrm{(95 \%} \mathrm{Cl}$ \\
\hline
\end{tabular}




\begin{tabular}{|c|c|c|c|}
\hline & $\begin{array}{l}\text { lifestyle and } \\
\text { dietary } \\
\text { interventions }\end{array}$ & care & $\begin{array}{l}0.53-0.83 \text { ) } \\
=1 \mathrm{OM} \text { less hypertension RR } 0.34 \text { (95\% Cl 0.13- } \\
0.91) \\
\text { No differences in GDM, C-section or high } \\
\text { birthweight } \\
\text { Small sample sizes }\end{array}$ \\
\hline $\begin{array}{l}\text { Kapadia, } \\
2015^{28}\end{array}$ & $\begin{array}{l}\text { Systematic } \\
\text { review of relation } \\
\text { between } \\
\text { psychological } \\
\text { factors and } \\
\text { trimester specific } \\
\text { GWG }\end{array}$ & $\begin{array}{l}2 \text { cohort studies, } n=1001 \\
\text { primary and secondary } \\
\text { care }\end{array}$ & $\begin{array}{l}\text { Distress was negatively associated with first } \\
\text { trimester GWG. There were inconclusive } \\
\text { findings regarding body image dissatisfaction } \\
\text { and trimester-specific GWG, with one of the } \\
\text { two studies showing an association with } \\
\text { second trimester GWG only among non- } \\
\text { adolescents. No association emerged between } \\
\text { perceived stress and state and trait anxiety and } \\
\text { trimester-specific GWG in a single study. The } \\
\text { relation between a number of weight-related } \\
\text { and dietary related cognitions, affective states } \\
\text { and personality traits and trimester-specific } \\
\text { GWG remains unexplored. }\end{array}$ \\
\hline $\begin{array}{l}\text { Kapadia, } \\
2015^{29}\end{array}$ & $\begin{array}{l}\text { Systematic } \\
\text { review of relation } \\
\text { between } \\
\text { psychological } \\
\text { factors and GWG }\end{array}$ & $\begin{array}{l}25 \text { cohort, } 8 \text { cross- } \\
\text { sectional and } 2 \text { case- } \\
\text { control studies, } 4 \text { studies } \\
n<100 ; 14 \text { studies } n=101- \\
400 ; 6 \text { studies } n=401- \\
1000 ; 11 \text { studies } n=1001- \\
10,000 \\
\text { primary and secondary } \\
\text { care }\end{array}$ & $\begin{array}{l}\text { Depression, anxiety, stress not related to GWG } \\
\text { >IOM related to concern about GWG, negative } \\
\text { body image, attitude towards GWG, inaccurate } \\
\text { perception regarding weight, higher target } \\
\text { GWG, less knowledge, higher levels of cognitive } \\
\text { dietary restraint, perceived barriers to healthy } \\
\text { eating } \\
\text { Protective factors for >IOM: internal locus of } \\
\text { control for weight gain, target lower than } \\
\text { recommended, higher se for healthy eating } \\
\text { Authors developed a pinwheel to give an } \\
\text { overview of the psychological antecedents of } \\
\text { GWG. }\end{array}$ \\
\hline $\begin{array}{l}\text { Lau, } \\
2014^{21}\end{array}$ & $\begin{array}{l}\text { Systematic } \\
\text { review of } \\
\text { association } \\
\text { between GWG } \\
\text { and bodyweight } \\
\text { of offspring (age } \\
2-18 \mathrm{yr} \text { ) }\end{array}$ & $\begin{array}{l}23 \text { cohort studies, } 1 \text { study } \\
n=100-400,2 \text { studies } \\
n=401-1000 ; 16 \text { studies } \\
n=1001-10,000,4 \text { studies } \\
n>10,000 \\
\text { primary and secondary } \\
\text { care }\end{array}$ & $\begin{array}{l}\text { >IOM more child overweight and obesity, } \\
\text { higher BMI z-scores }\end{array}$ \\
\hline $\begin{array}{l}\text { Mamun, } \\
2014^{25}\end{array}$ & $\begin{array}{l}\text { Systematic } \\
\text { review and meta- } \\
\text { analysis of risk of } \\
\text { obesity in } \\
\text { offspring (three } \\
\text { stages) when } \\
\text { GWG was too } \\
\text { high or too low }\end{array}$ & $\begin{array}{l}15 \text { studies for review } \\
\text { included, } 12 \text { for meta- } \\
\text { analysis }(n=93,711), 1 \\
\text { study } n=100-400,1 \text { study } \\
n=401-1000,7 \text { studies } \\
n=1001-10,000,6 \text { studies } \\
n>10,000 \\
\text { primary and secondary } \\
\text { care }\end{array}$ & $\begin{array}{l}<\text { IOM RR } 0.86 \text { ( } 95 \% \mathrm{Cl} 0.78-0.94) \text { for obesity in } \\
\text { offspring in all stages } \\
>\text { IOM RR } 1.40 \text { ( } 95 \% \mathrm{Cl} 1.23-1.59) \text { for obesity in } \\
\text { offspring in all stages }\end{array}$ \\
\hline $\begin{array}{l}\text { Ruifrok, } \\
2014^{31}\end{array}$ & $\begin{array}{l}\text { Meta-analysis on } \\
\text { association } \\
\text { between GWG }\end{array}$ & $\begin{array}{l}23 \mathrm{RCT}^{\prime} \text { s (lifestyle } \\
\text { interventions), } \mathrm{n}=4,990 \\
\text { primary and secondary }\end{array}$ & $\begin{array}{l}\text { Non-significant effects of GWG on PE, GDM } \\
\text { and induction of labour }\end{array}$ \\
\hline
\end{tabular}




\begin{tabular}{|c|c|c|c|}
\hline & $\begin{array}{l}\text { and rate of } \\
\text { pregnancy } \\
\text { complications }\end{array}$ & care & \\
\hline $\begin{array}{l}\text { Nehring, } \\
2013^{26}\end{array}$ & $\begin{array}{l}\text { Meta-analysis on } \\
\text { relation between } \\
\text { GWG and } \\
\text { childhood } \\
\text { overweight }\end{array}$ & $\begin{array}{l}7 \text { observational studies, } 1 \\
\text { study } n=400-1000,3 \\
\text { studies } n=1000-10,000,3 \\
\text { studies } n>10,000 \\
\text { primary and secondary } \\
\text { care }\end{array}$ & $\begin{array}{l}\text { <IOM: OR } 0.91(95 \% \mathrm{Cl} 0.85-0.98) \text { childhood } \\
\text { over-weight } \\
\text { >IOM: OR } 1.38(95 \% \mathrm{Cl} 1.21-1.57) \text { childhood } \\
\text { over-weight }\end{array}$ \\
\hline $\begin{array}{l}\text { Adamo, } \\
2012^{22}\end{array}$ & $\begin{array}{l}\text { General review } \\
\text { of literature on } \\
\text { intergenerational } \\
\text { cycle of obesity }\end{array}$ & 269 references & $\begin{array}{l}\text { Association between healthy maternal pre- } \\
\text { pregnancy BMI and offspring obesity. Direct } \\
\text { association between GWG and birth weight or } \\
\text { infant adiposity, maybe genetic and } \\
\text { environmental contributions as confounding } \\
\text { factors. Higher GWG is associated with } \\
\text { increased risk of GDM and foetal } \\
\text { hyperglycaemia. }\end{array}$ \\
\hline $\begin{array}{l}\text { Poston, } \\
2012^{23}\end{array}$ & $\begin{array}{l}\text { General review } \\
\text { of literature on } \\
\text { effect of diet rich } \\
\text { in calories on risk } \\
\text { of obesity in } \\
\text { offspring }\end{array}$ & 118 references & $\begin{array}{l}\text { Maternal obesity and higher GWG are } \\
\text { associated with increased risk of obesity in } \\
\text { offspring. Studies in rodents and their } \\
\text { genetically identic twins suggest pathways } \\
\text { through intrauterine environment. }\end{array}$ \\
\hline $\begin{array}{l}\text { Poston, } \\
2012^{24}\end{array}$ & $\begin{array}{l}\text { General review } \\
\text { to relate too high } \\
\text { GWG with } \\
\text { offspring health }\end{array}$ & 37 references & $\begin{array}{l}\text { Recent studies report relationships between } \\
\text { excessive GWG and neonatal adiposity, and } \\
\text { with childhood and adult obesity. These appear } \\
\text { to be independent of confounders such as } \\
\text { socioeconomic status and a shared family } \\
\text { environment, or hereditary traits for obesity, } \\
\text { supporting the 'developmental origins of } \\
\text { disease' hypothesis. Author present theoretical } \\
\text { pathways leading to childhood obesity arising } \\
\text { from excessive GWG }\end{array}$ \\
\hline $\begin{array}{l}\text { Han, } \\
2011^{30}\end{array}$ & $\begin{array}{l}\text { Systematic } \\
\text { review and meta- } \\
\text { analysis on } \\
\text { relation between } \\
\text { low GWG and } \\
\text { preterm birth }\end{array}$ & $\begin{array}{l}37 \text { cohort studies and } 18 \\
\text { case-control studies } \\
n=3,467,638 \\
\text { primary and secondary } \\
\text { care }\end{array}$ & $\begin{array}{l}\text { Low weekly GWG RR for low birthweight } 1.64 \\
\text { (95\% Cl 0.89-3.02) } \\
\text { Low weekly GWG RR for preterm birth } 1.56 \\
\text { (95\% Cl 1.26-1.94) } \\
\text { Low total GWG RR for low birthweight } 1.85 \\
\text { (95\% Cl 1.72-2.00) } \\
\text { Low total GWG RR for preterm birth } 1.64 \text { (95\% } \\
\mathrm{Cl} 1.62-1.65 \text { ) } \\
\text { The lower the GWG, the higher the risks. }\end{array}$ \\
\hline $\begin{array}{l}\text { McDonald, } \\
2011^{27}\end{array}$ & $\begin{array}{l}\text { Review and } \\
\text { meta-analysis on } \\
\text { relationship } \\
\text { between high } \\
\text { GWG and } \\
\text { preterm birth } \\
\text { and low birth } \\
\text { weight }\end{array}$ & $\begin{array}{l}38 \text { studies } n=2,124,907 \\
\text { primary and secondary } \\
\text { care }\end{array}$ & $\begin{array}{l}\text { >IOM: RR for preterm birth } 0.75(95 \% \mathrm{Cl} 0.60- \\
0.96) \text { and for low birth weight RR } 0.64(95 \% \mathrm{Cl} \\
0.53-0.78) \\
\text { High weekly GWG: RR for preterm birth } 1.51 \\
(95 \% \mathrm{Cl} 1.47-1.55)\end{array}$ \\
\hline
\end{tabular}




\begin{tabular}{|c|c|c|c|}
\hline $\begin{array}{l}\text { Nehring, } \\
2011^{20}\end{array}$ & $\begin{array}{l}\text { Meta-analysis on } \\
\text { relation between } \\
\text { GWG and long- } \\
\text { term risk of over- } \\
\text { weight in women }\end{array}$ & $\begin{array}{l}9 \text { observational studies, } 2 \\
\text { studies } n=101-400 ; 1 \\
\text { study } n=401-1000 ; 4 \\
\text { studies } n=1001-10,000 ; 2 \\
\text { studies } n>10,000 \\
\text { primary and secondary } \\
\text { care }\end{array}$ & $\begin{array}{l}>I O M: 4.29 \mathrm{~kg}(95 \% \mathrm{Cl} 3.15-5.43) \text { after } 0.5 \mathrm{yr} \text {; } \\
2.45 \mathrm{~kg}(1.95-2.95) \text { after } 0.5-1 \mathrm{yr} ; 3.06 \mathrm{~kg}(95 \% \\
\mathrm{Cl} 1.50-4.63) \text { after } 3 \mathrm{yr} \text { and } 4.72 \text { (95\% } \mathrm{Cl} 2.94- \\
6.50) \text { after } 15 \mathrm{yr} \\
<10 \mathrm{O}:-2.99 \mathrm{~kg}(95 \% \mathrm{Cl} 3.72 \text { to }-2.27) \text { after } 0.5 \\
\mathrm{yr} ;-2.06(95 \% \mathrm{Cl}-2.45 \text { to }-1.67) \text { after } 0.5-1 \mathrm{yr} ;- \\
2.08 \mathrm{~kg}(95 \% \mathrm{Cl}-2.85 \text { to }-1.31) \text { after } 3 \mathrm{yrs} ;-1.41 \\
\mathrm{~kg}(95 \% \mathrm{Cl}-3.03 \text { to } 0.21) \text { after } 15 \mathrm{yr}\end{array}$ \\
\hline $\begin{array}{l}\text { Siega-Riz, } \\
2009^{18}\end{array}$ & $\begin{array}{l}\text { Systematic } \\
\text { review on } \\
\text { obstetric } \\
\text { outcomes } \\
\text { (birthweight, } \\
\text { fetal growth and } \\
\text { PPWR) related to } \\
\text { GWG }\end{array}$ & $\begin{array}{l}61 \text { comparisons in } 35 \\
\text { studies, } 1 \text { study } n<100,9 \\
\text { studies } n=101-400 ; 16 \\
\text { studies } n=401-1000 ; 16 \\
\text { studies } n=1001-10,000 ; \\
18 \text { studies } n>10,000,1 \\
\text { study not reported } \\
\text { primary and secondary } \\
\text { care }\end{array}$ & $\begin{array}{l}\text { <IOM1990 more sga, low birth weight, preterm } \\
\text { >IOM1990 more Iga, macrosomia, PPWR }\end{array}$ \\
\hline $\begin{array}{l}\text { Viswanathan, } \\
2008^{19}\end{array}$ & $\begin{array}{l}\text { General } \\
\text { systematic } \\
\text { review on } \\
\text { outcomes of } \\
\text { GWG and } \\
\text { confounders and } \\
\text { effect modifiers }\end{array}$ & $\begin{array}{l}150 \text { studies } \\
\text { primary and secondary } \\
\text { care }\end{array}$ & $\begin{array}{l}\text { <IOM1990 more preterm (strong evidence), } \\
\text { more sga (strong evidence), low birthweight } \\
\text { (strong evidence), lower likelihood to initiated } \\
\text { breastfeeding (moderate evidence) } \\
\text { >IOM1990 more preterm (strong evidence), } \\
\text { higher PPWR (moderate evidence), more C- } \\
\text { section (moderate evidence), more Iga (strong } \\
\text { evidence), more macrosomia (strong } \\
\text { evidence), short-term PPWR (moderate } \\
\text { evidence), intermediate PPWR (moderate } \\
\text { evidence), long term PPWR (moderate } \\
\text { evidence) }\end{array}$ \\
\hline
\end{tabular}

Abbreviations: GDM gestational diabetes mellitus; GWG gestational weight gain; IOM, Institute of Medicine guidelines date 2009; IOM1990 Institute Of Medicine weight gain guidelines date 1990; IPV, Intimate Partner Violence; Iga large for gestational age; PE pre-eclampsia; PPWR post-partum weight retention; RCT randomised controlled trial; RR relative risk; se, self-efficacy; sga small for gestational age; USA, United States of America

As this compilation of reviews makes clear, the negative outcomes associated with excessive and inadequate GWG makes investment in promoting healthy GWG worthwhile.

\subsection{Prevalence of women with healthy gestational weight gain}

The percentage of women with a healthy GWG (GWG within the IOM-guidelines) varies worldwide from $18.9 \%$ to $51.9 \%{ }^{2,41-43}$ depending largely on characteristics of the studied population, such as their BMI category. In a Dutch study of 144 women, done by Althuizen et al., the percentage of women with healthy GWG varied between $25 \%$ for overweight women and $49 \%$ for normal weight women. ${ }^{44}$ The majority of women with an unhealthy GWG gained too much weight. For normal weight women this was $31 \%$ and for overweight women this was $62 \%{ }^{44}$ Another Dutch study, of a cohort of 1343 healthy women, reported that 
percentages of women having a healthy GWG varied from $29 \%$ in obese women to $42 \%$ in normal weight women. ${ }^{45}$ In this group, too high GWG varied from $13 \%$ in normal weight women to $56 \%$ in obese women. ${ }^{45}$ The differences in the numbers can be explained by the way of the calculations used (self-reported pre-pregnancy weight and self-reported weight at the end of pregnancy versus measured weight at 12 and 36 weeks gestation) and differences in the population (women sampled from the general Dutch population versus specific target groups, e.g., healthy Caucasian pregnant women). Furthermore, the Althuizen et al. study used the IOM-guidelines from 1990, which are different from the BMI categories in the IOMguidelines of 2009. ${ }^{6,44}$ A study comparing the application of the 1990 guidelines and the 2009 guideline to the same data, reported considerable shifts in classifying BMI category and therefore in the healthy GWG classification. ${ }^{42}$ Findings from a third Dutch study among 6012 pregnant women revealed that one third gained too much weight. ${ }^{46}$ In summary, somewhere between a quarter and a half of all Dutch women gain weight within the IOM-guidelines, with 13 to $62 \%$ gaining too much weight. This makes it critical to invest in the development and testing of interventions that will increase the number of women with a healthy GWG.

\subsection{Risk factors for unhealthy gestational weight gain}

Factors contributing to both excessive and inadequate GWG, can be subdivided into three categories: personal characteristics, modifiable factors and other. The first category is important because it allows the identification of women who have a higher risk for unhealthy GWG; the second category is equally important because these are the factors that can be targeted by an intervention. The third category is important, but as these are difficult, if not impossible to modify, they are not the proper focus of an intervention program. In choosing this way of dividing risk factors we are focusing on those aspects we believe are possible for caregivers to change by means of an intervention. For example, although a low level of education is a modifiable factor in general, for a clinician it is a feature that is impossible to change.

Personal characteristics that are associated with a higher prevalence of excessive GWG are ethnicity, - with black women more likely to have excessive GWG, ${ }^{47,48}$-, low level of education, ${ }^{49,50}$ having a low income, ${ }^{51}$ being nulliparous, ${ }^{52}$ "stopped smoking" status, being older, high birthweight of the mother ${ }^{53}$ and "hardship" in childhood (defined as lacking basic necessities like food, rent or medical care). ${ }^{54}$ Women who had fertility treatment are less likely to have excessive GWG. ${ }^{53}$ Factors associated with inadequate GWG are intimate partner violence (IPV), ${ }^{55}$ being older, having a low income, being black or Hispanic, being unmarried and a low level of education. ${ }^{6,56,57}$

Factors associated with excessive GWG that are modifiable by caregivers are diet and physical activity, ${ }^{6,51,53,58}$ having a too high goal for $\mathrm{GWG}^{59,60}$ a higher $\mathrm{BMI}^{44,50,53,61}$ and increased hours of sleep. ${ }^{53}$ For inadequate GWG these factors are having a too low goal for $\mathrm{GWG}^{59}$ having a low BMI, and smoking., ${ }^{6,56}$ 57 


\subsection{Aim of this thesis}

Reviewing the literature on GWG, it became clear that both excessive and inadequate GWG need to be prevented, for women in all BMI categories. Accordingly, the main research question of this thesis is:

Can we develop an evidence based intervention to support Dutch primary care midwives in the effective promotion of a healthy GWG for women in their care?

To answer this question, we formulated the following sub questions:

1. What are the existing evidence-based prenatal interventions for promoting a healthy GWG?

2. What personal characteristics and behavioural factors of pregnant women are related to the achievement of healthy GWG?

3. What are Dutch midwives currently doing to encourage a healthy GWG for their clients?

4. What systematically developed prenatal intervention will promote healthy GWG in midwife-led care?

5. Will an evidence based prenatal intervention promote healthy GWG?

\subsection{Theoretical framework}

We chose Intervention Mapping (IM) as the framework for the project. IM is a protocol for developing evidence-informed interventions to promote changes in health behaviours. ${ }^{62} \mathrm{IM}$ has six steps. The first three steps focus on preparation: Step 1 is a needs assessment, after which program objectives are formulated (step 2), followed by the selection of theory based intervention methods to reach the objectives (step 3). Subsequently, the program components and materials are developed (step 4) and an implementation plan is formulated (step 5). The final step is the design of an evaluation plan (step 6), which makes the intervention ready to implement in practice. Intervention Mapping is iterative: at each step and at the conclusion of the project, results are evaluated and fed back into the process (Figure 1.1).

\subsection{How to read this thesis}

As you read through this thesis, you will find the answers to the research questions posed above. Chapter 2 describes a review and meta-analyses of the intervention studies aimed at influencing GWG that were published up until 2011. Chapter 3 describes the GWG in a sample of 455 healthy pregnant women in the Netherlands in various stages of their pregnancy, related to the IOM-guidelines. The study includes an analysis of factors associated with GWG, including diet and changes in level and types of physical activity (PA). The focus of chapter 4 is on the change in PA, an important factor in promoting healthy GWG. Chapter 5 describes a qualitative study among a purposive sample of six primary care midwives. We examined their behaviour in promoting healthy GWG in pregnant women as well as the determinants of 
those behaviours. The results of this study served as a basis for a cross-sectional survey among 112 primary care midwives, described in chapter $\mathbf{6}$. Chapter 7 outlines the development of our intervention. We used what we learned in the studies described in chapters 2 through 6 , together with information from literature, to create an innovative intervention for both pregnant women and midwives. In chapter 8 we present the results of our pilot study of the intervention. We conclude the thesis with a general discussion of the main findings and consideration of the implications of these findings for practice and future research (chapter $\mathbf{9}$ ).

To enhance readability, some tables and one figure are printed at the end of the thesis.

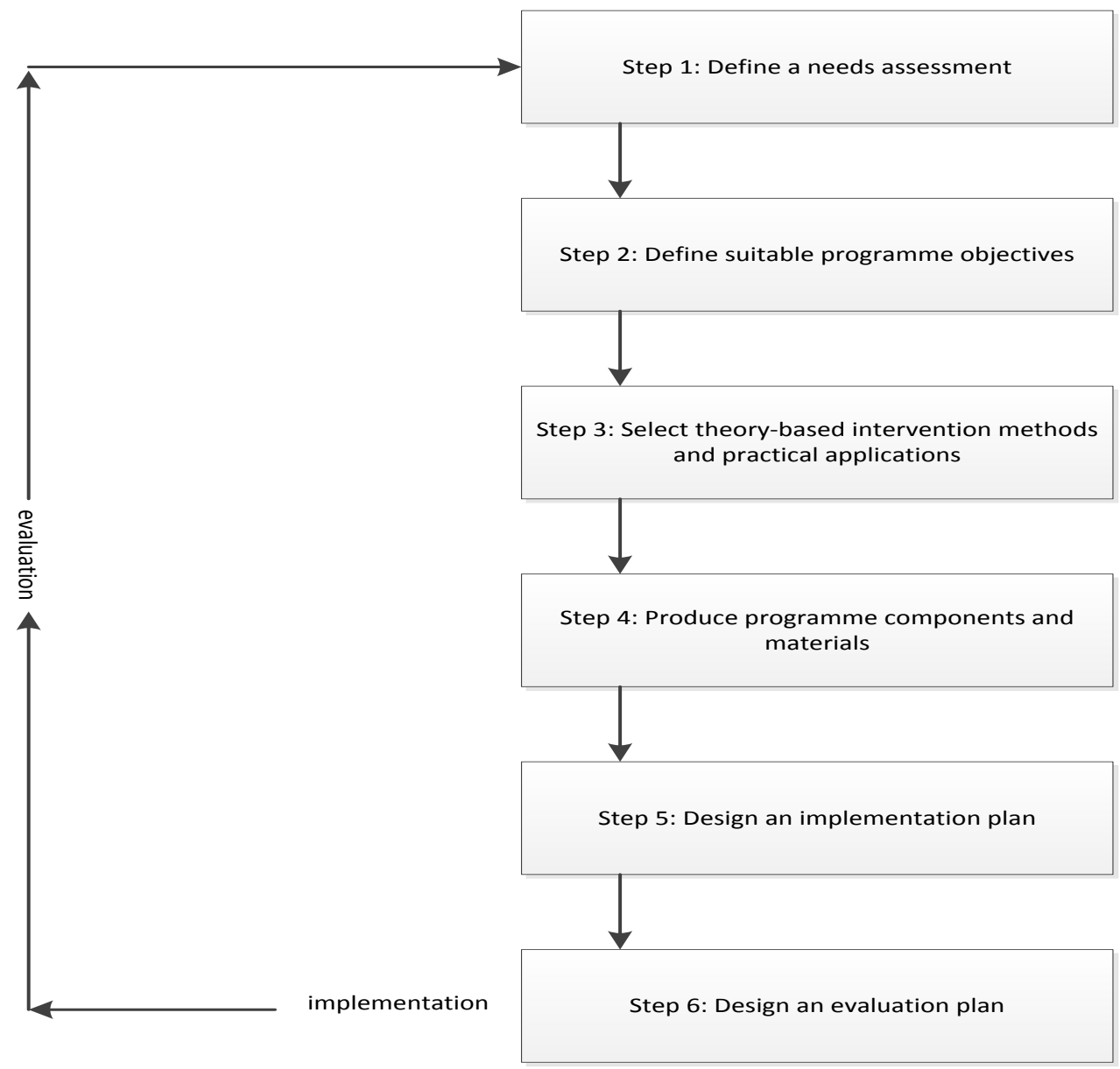

Figure 1.1 Intervention Mapping (IM) process (source Bartholomew et al. 2011) 


\section{Chapter 2}

\section{Interventions aiming to achieve a healthy gestational weight gain: A systematic review / meta-analysis}

Astrid Merkx, Caroline H.G. Bastiaenen, Cecile van Lümig, Marlein Ausems, Marianne J. Nieuwenhuijze, Raymond de Vries 


\section{Background}

A majority of pregnant women have a too high or too low gestational weight gain (GWG).

Objective

A systematic review and meta-analysis to assess relevant characteristics and outcomes of interventions for pregnant women with the aim of achieving a healthy GWG.

\section{Search strategy}

Relevant databases were searched for interventions to promote healthy GWG. Handsearching in relevant journals and reference lists of studies and reviews (Last search 30 September 2011).

\section{Selection criteria}

Randomised Controlled Trials (RCT) and non-RCT's with the primary outcome of healthy GWG or percentage of pregnant women gaining weight within guidelines, published in 2001 or later. No restriction in language.

\section{Data collection and analysis}

Twenty-eight studies were found, with 7410 participants. After quality assessment, 14 RCT's and one non-RCT remained; three groups of interventions were formed for a meta-analysis: dietary interventions for obese women, physical activity (PA) for all women and combined interventions for all women.

\section{Main results}

Most of the studies came from the United States of America and Europe. Half of the interventions focused on a single theme, the other half combined diet, PA and weightmonitoring. In the meta-analysis diet for obese women resulted in a mean difference of -8.41 $\mathrm{kg}(95 \% \mathrm{Cl}-10.49,-6.34 \mathrm{~kg})$, PA resulted in a mean difference of $-0.83 \mathrm{~kg}(95 \% \mathrm{Cl}-1.47,-0.19$ $\mathrm{kg}$ ) and three multiple content interventions had no significant effects. However, it is not known whether women in these studies gained weight within guidelines. Secondary outcomes were inconsistent.

\section{Conclusions}

GWG for obese women can be reduced with interventions focusing on diet and on PA. 


\subsection{Introduction}

Too high GWG and too low GWG contribute directly and indirectly to complications in pregnancy and birth, including preterm birth and SGA, which in turn contribute to higher perinatal death rates. ${ }^{18,19}$ GWG above the guidelines is associated with overweight in the long term for mothers and babies. Excessive GWG is associated with a higher likelihood of gestational diabetes mellitus (GDM) and foetal hyperglycaemia, ${ }^{22}$ Caesarean section, ${ }^{19}$ preterm birth, ${ }^{19,27}$ and negative psychological outcomes. ${ }^{28,}{ }^{29}$ Inadequate GWG is associated with a higher likelihood of low birthweight ${ }^{18,19,30}$ and preterm birth, ${ }^{18,19,30}$ and a lower likelihood to initiate breastfeeding. ${ }^{19}$

In 2009, the Institute of Medicine (IOM) published guidelines with recommendations for appropriate GWG according to a woman's pre-pregnancy Body Mass Index (BMI). ${ }^{6}$ Between 18.9 and $51.9 \%$ of pregnant women in high-resource countries gain weight within these guidelines $\mathrm{GWG}^{2,41-43}$. Professionals who work with pregnant women have a great need for an effective intervention that will help women achieve a healthy GWG. Because developing an effective intervention is time-consuming and expensive, it is imperative that it is based on sound research. A necessary first step in developing such an intervention is reviewing the successes and failures of existing interventions.

Reviews of interventions for helping women achieve appropriate GWG do exist, but they are not systematic, ${ }^{6,47,63,64}$ they focus on particular groups of participants, ${ }^{65-71}$ they focus on specific interventions such as diet ${ }^{63,72-76}$ or they do not use evidence from newly-developed evaluation studies of prevention of excessive GWG. ${ }^{77,78}$ Existing reviews do not clearly identify interventions that lead to a healthy GWG and new studies are continuously being published. Therefore it is time for a new systematic review and meta-analysis of the characteristics and treatment effects of interventions that promote a healthy GWG. Our goal is to describe current knowledge and measure the primary and secondary outcomes about all possible interventions for achieving a healthy GWG in pregnant women.

\subsection{Methods}

Our review followed the methods described in the Cochrane Handbook. ${ }^{79}$

\subsubsection{Search}

We conducted our search between 1 May 2011 and 30 September 2011. We searched the following sources for intervention studies:

1. Pubmed, EMBASE, Cochrane, Psychinfo, Web of Science and CINAHL databases. We used this search terms: (health promotion OR primary prevention OR secondary prevention OR intervention) AND (gestational weight gain OR pregnancy weight gain OR maternal weight gain) AND (pregnant OR pregnancy OR gestation).

2. Reference lists of reviews concerning interventions on GWG.

3. Reference lists of the studies included. 
4. Regularly recurring author names were used to search in Pubmed, EMBASE, Psychinfo and CINAHL.

5. Archives of relevant journals were hand-searched: BMC Pregnancy and Childbirth, BMC Public Health, American Journal of Obstetrics and Gynecology, BJOG, Nutrition and dietetics, Journal of Women's Health, European Journal of Obesity, Midwifery, Acta Obstetricia et Gynecologica Scandinavica; These were chosen because we regularly encountered these journals in the search process.

We searched for ongoing studies in Randomised Controlled Trial (RCT) databases: www.clinicaltrials.gov, www.controlled-trials.com, http://www.who.int/ictrp/en and http://www.anzctr.org.au.

\subsubsection{Inclusion and exclusion criteria}

All intervention studies with the following characteristics were included:

1. Published in $\mathbf{2 0 0 1}$ or later

2. Primary outcome was GWG and/or the percentage of women gaining weight within the IOM-guidelines.

Secondary outcomes included pregnancy-related outcomes (e.g., pregnancy induced hypertension (PIH) and gestational diabetes mellitus (GDM)), birth outcomes (e.g., preterm birth, need for induction of labour), neonatal outcomes (e.g., Apgar scores, birth weight) and mother related outcomes (e.g., post-partum depressions, weight retention). All secondary outcomes were taken into account.

We used no restrictions for language, population, design or countries. Two authors (AM and $\mathrm{CL}$ ) independently searched and screened the search results by reading titles and summaries. Disagreements about inclusions were resolved by discussions between those two authors.

\subsubsection{Methodological quality assessment}

Two authors ( $\mathrm{AM}$ and $\mathrm{CL}$ ) evaluated the RCT's independently using the following approach. First, we assessed all studies with the 25-item list from Consolidated Standards of Reporting Trials (CONSORT) ${ }^{80,81}$ Authors of the CONSORT list recommend it be used to gain objective insight into a study's methodological quality; they do not recommend simply counting the sum score of the list. ${ }^{80,81}$ Parts of the CONSORT list were used to fill in the Review Manager (REVman), ${ }^{79}$ a short list for creating an overview of the main characteristics of the study. We used the 11-item Physiotherapy Evidence Database quality (PEDro) list ${ }^{82}$ to set a cut-off point for the methodological quality of a study. PEDro gives credits for 11 important elements of methodological quality such as randomisation and blinding. A study can score between 11 points (highest quality) and zero points (lowest quality). The PEDro cut-off point is not prescribed because some items on the list are not relevant for every study. ${ }^{82,83}$ In our case for instance, it is not possible to double-blind a study when women are asked to do exercises. In order to set the PEDro cut-off point for good quality RCT's, we looked at the consistency of the REVman and the PEDro list together. Consistency was highest when we placed the cut-off point between the score of six and seven. ${ }^{82,83}$ 
The methodological quality of non-RCT studies was assessed by the same two authors using the approach developed by the Grades of Recommendation, Assessment, Development and Evaluation (GRADE) Working Group. ${ }^{79}$ In this approach the quality of a well-conducted observational study can be upgraded when there is absence of confounders, when the study effect is likely to be larger than reported or when the study provides a dose-response gradient. A study can be downgraded because of methodological issues such as design limitations, evidence of indirect effects or imprecise results. According to the Cochrane Handbook a double upgraded (and not downgraded) observational study meets the quality of a well-conducted $\mathrm{RCT}^{79}$ which means that RCT's and non-RCT's can be compared under strict conditions. Therefore we decided to present the primary and secondary outcome of observational studies that would be graded as "high quality" together with the results of the RCT's with a PEDro score of seven or higher.

Authorship was not blinded for the assessors. In the event of disagreements between the two assessors, a third author with experience in systematic reviews (CB) was consulted and consensus was reached.

\subsubsection{Data synthesis}

Our primary outcome was difference in mean GWG. We assessed all studies on their clinical homogeneity; that is, use of the same sort of intervention for the same target group. In order to assess the statistical heterogeneity of high quality studies that we considered for pooling we calculated $\mathrm{I}^{2}$, a measure of statistical heterogeneity. ${ }^{79}$ To do so, we first had to calculate the standard error (SE) of one study ${ }^{84}$ which we did using the tables of probability distributions. ${ }^{85}$ For another study, ${ }^{86}$ we needed to calculate the mean weight gain for two different groups because the author provided results on normal pre-pregnancy BMI and on pre-pregnancy BMI above $25 \mathrm{~kg} / \mathrm{m}^{2}$. For two studies ${ }^{84,87}$ we calculated the standard deviation (sd) out of the SE with the following equation: $s d=S E * V N$ (N=total of participants). ${ }^{79} \mathrm{We}$ followed the Cochrane Handbook's suggestion to set the cut-off point of $\mathrm{I}^{2} ; \mathrm{I}^{2}>40 \%$ indicates a level of heterogeneity that is too high to allow pooling. ${ }^{79}$ We chose to pool with the random effects model when $\mathrm{I}^{2}$ was $0-40 \%$ and the fixed effects model when $\mathrm{I}^{2}$ was zero to estimate the total effect of the interventions. ${ }^{79}$ For the remaining heterogenic studies, we described the outcomes narratively.

\subsection{Results}

Our search for eligible studies identified 742 titles of which 161 were duplicates and 552 were excluded, leaving 29 titles. Of these 29 titles, 19 were RCT's ${ }^{84,86-103}$ and nine were nonRCT's. ${ }^{104-112}$ One study was presented at a conference, but further publication is missing so we could not assess it. ${ }^{113}$ An overview of the search results is shown in Figure 2.1.

All published studies of RCT's and non-RCT's were included for systematic analysis $(n=28)$. 


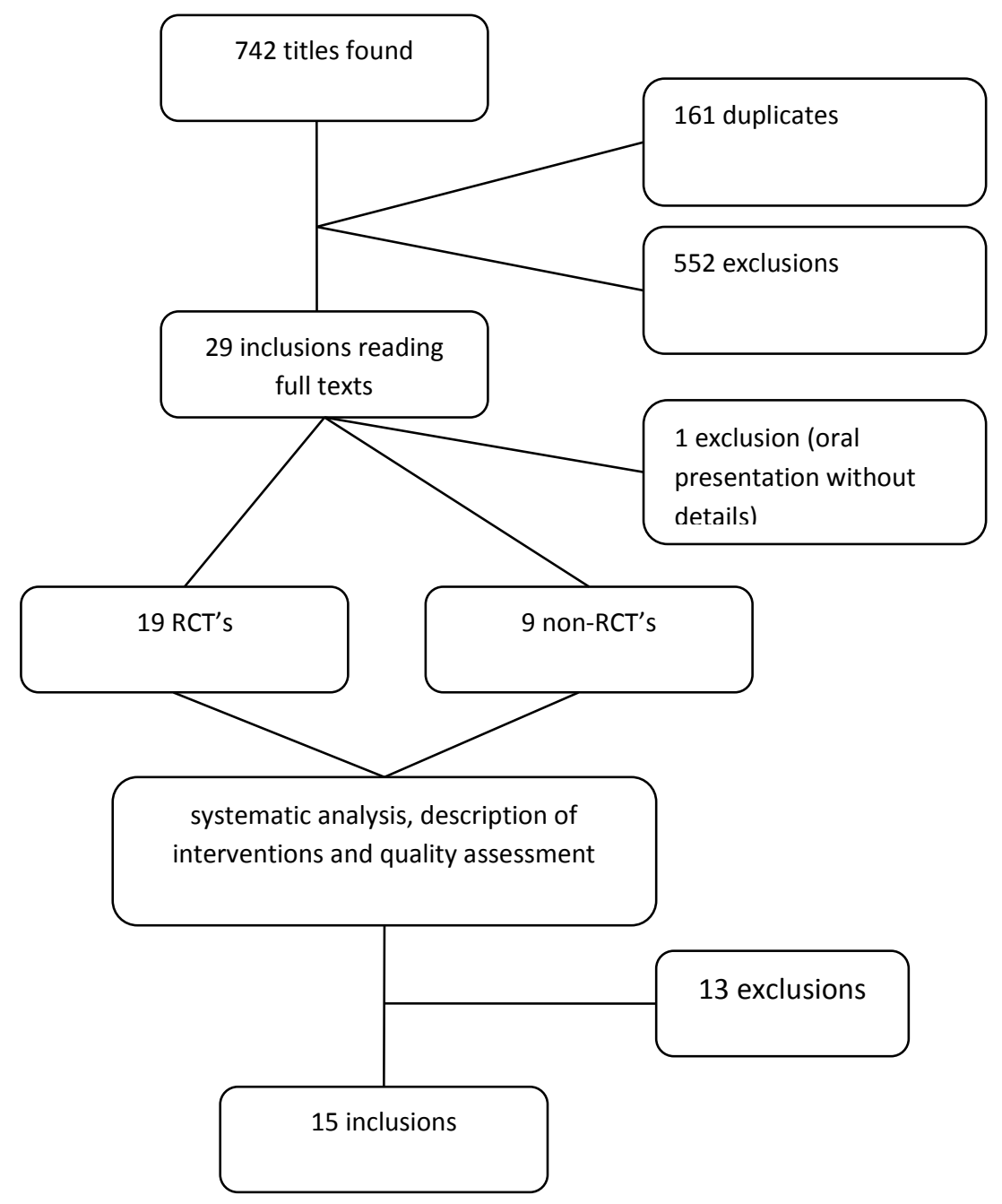

Figure 2.1 Flowchart of studies included

\subsubsection{Study participants}

In total, 7410 participants were involved in the 28 studies, with a study size range of 41 to 1928 participants. Twelve studies were carried out in the United States of America, ${ }^{84,88,96-98,}$ 100, 103, 105, 106, 109, 110, 112 three in Canada, ${ }^{86,93,111}$ eight in Europe (Finland, ${ }^{94,95,104}{ }^{\text {Norway, }}{ }^{102}$ Sweden, ${ }^{107}$ Denmark, ${ }^{99}$ Belgium $^{92}$ and Spain ${ }^{90}$ ), two in Brazil, ${ }^{89,91}$ two in Australia ${ }^{87,} 101$ and one in India. ${ }^{108}$

Two studies focused on women with GDM, ${ }^{89,95}$ and one on obese women with GDM. ${ }^{106}$ Five studies included only obese women, ${ }^{92,99,103,105,107}$ four studies included both overweight and obese women, ${ }^{87,96,97,111}$ one study included normal weight and overweight women, ${ }^{112}$ and 
one included primipara normal weight and over-weight women. ${ }^{104}$ One study focused on African-American teenagers, ${ }^{100}$ one included only women in the first trimester with nausea and vomiting, ${ }^{98}$ two focused on sedentary pregnant women, ${ }^{91,102}$ four focused on low income women, ${ }^{90,108-110}$ and the other six focused on pregnant women in general. ${ }^{84,86,88,93,94,101}$ Not including the study of teenagers ${ }^{100}$ and the one study that did not report participants' ages, ${ }^{101}$ the mean age varied between 21.8 and 33.7 years. Ethnic background varied: three of the studies' participants were all Caucasian ${ }^{90,92,100}$ one study's participants were all AfricanAmerican ${ }^{100}$ and twelve other studies reported different combinations of ethnic backgrounds. ${ }^{84,86-88,93,96-98,103,105,106,110}$ Ten studies did not mention ethnicity.

Social-economic status was reported in eleven studies in different ways (e.g., percentage of employment, percentage of insurance with Medicaid and income). ${ }^{84,86,90,93,96,105,107-110,112}$ Thirteen studies reported education level, ${ }^{84,86,90,91,93-95,97,102,104,108,110,112}$ which was determined by reporting years of education, or the highest level of education completed. Education cut-off points varied between studies, but in one study it was unclear. ${ }^{88}$ Physical activity (PA) was reported as a baseline characteristic in four studies. ${ }^{89-91,102}$

\subsubsection{Intervention characteristics}

None of the studies reported that their intervention was based on a systematic approach. One study mentioned that social learning theory underpinned their intervention, ${ }^{86}$ while seven studies indirectly mentioned that behavioural theories were used to develop their intervention. $^{88,92,93,96,105,107,109}$

Six interventions focused on diet (all in individual sessions), ${ }^{94,97,99,100,103,108}$ four interventions consisted of activities to increase PA ${ }^{89-91,102}$ and one intervention studied the influence of weighing and providing information on appropriate and current GWG. ${ }^{101}$ Fourteen

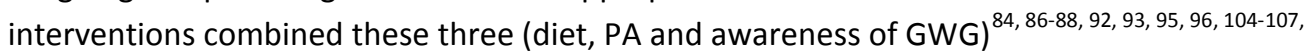
111,112 of which two included group sessions ${ }^{92,95}$ and the remaining included individual counselling sessions. The multiple-content interventions were all based on a client-centred holistic approach. Six of them used a phased approach (care depending on the needs) and eleven of the fourteen multiple-content interventions used a multi-channel approach (combining two or more information channels such as brochures, mail, telephone, video, email, home visits and personal contacts).

Three interventions were unique: one used a nerve stimulation band to decrease first trimester nausea; ${ }^{98}$ another compared women with and without a case manager ${ }^{110}$ and a third discussed health issues during group consults as compared to standard individual consults. $^{109}$

Support in the intervention varied from no support to supportive sessions in a small group three times a week. We must advise caution here as words like support are easily misinterpreted: support can be used for different content, such as "leading," or stimulating one's own leadership potential, or reinforcement strategies. The motivations of the professionals working with the intervention were not reported. Adherence to the program was reported in six studies and varied from $58 \%{ }^{100}$ to $100 \% .{ }^{86,95,101,108,112}$ 
Twelve studies were put together in three groups each with comparable interventions used in comparable target populations: two studies on diets for obese women, ${ }^{99,103}$ which we refer to as the "diet for obese" group; three studies on PA for all BMI groups, ${ }^{90,91,102}$ which we refer to as the "PA for all" group; and seven studies on multiple-content intervention for all BMI groups $^{84,86,88,93,96,104,112}$ which we refer to as the "multiple-content for all" group.

\subsubsection{Methodological quality and heterogeneity}

The methodological quality of the nineteen RCT's varied on the PEDro list from three to ten points out of eleven. Five studies were excluded because of a PEDro score of six or less. ${ }^{88,92,93 \text {, }}$ ${ }^{96,100}$ The results of the RCT assessments are shown in Table 2.1 (at the end of this thesis). The methodological quality of the nine non-RCT's varied from very low to high according to the GRADE classification Table 2.2 (at the end of this thesis). Eight studies were excluded because they could not be double upgraded. ${ }^{105-112}$

We considered pooling the high quality studies of the three groups of clinically homogenic intervention studies: 1) "Diet for obese" group, 2) "PA for all" group, and 3) "multiple-content for all" group. $\mathrm{I}^{2}$ of the pooled groups was $25 \%, 0 \%$ and $0 \%$ respectively, all of which are acceptable. We therefore used the random effects method for pooling the "diet for obese" group and the fixed effects method for pooling the other two groups.

\subsubsection{Primary outcome}

GWG in the "diet for obese" group resulted in a mean difference of $-8.41 \mathrm{~kg}$ ( $95 \% \mathrm{Cl}$; -10.49; 6.34 ) in the intervention group compared to the control group. The "PA for all" group had a mean difference of GWG of $-0.83 \mathrm{~kg}(95 \% \mathrm{Cl} ;-1.47 ;-0.19)$ in the intervention group, while the "multiple content for all" group had an insignificant mean difference of GWG of $-0.12 \mathrm{~kg}(95 \%$ $\mathrm{Cl}-0.54 ; 0.31$ in the intervention group. Pooling results are shown in Tables 2.3, 2.4 and 2.5.

Table 2.3 Result of the "diet for obese" studies

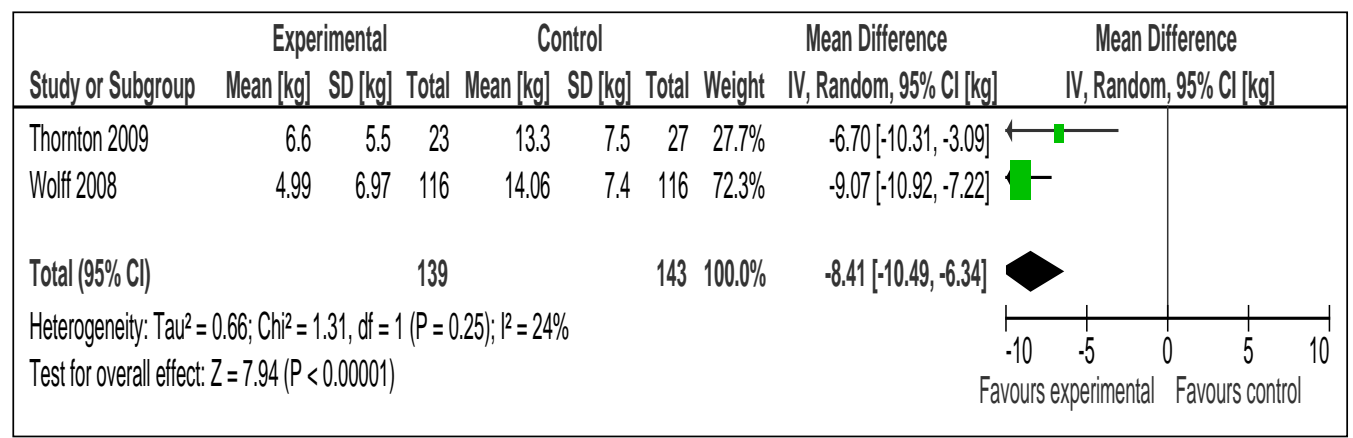


Table 2.4 Result of the "PA for all" studies

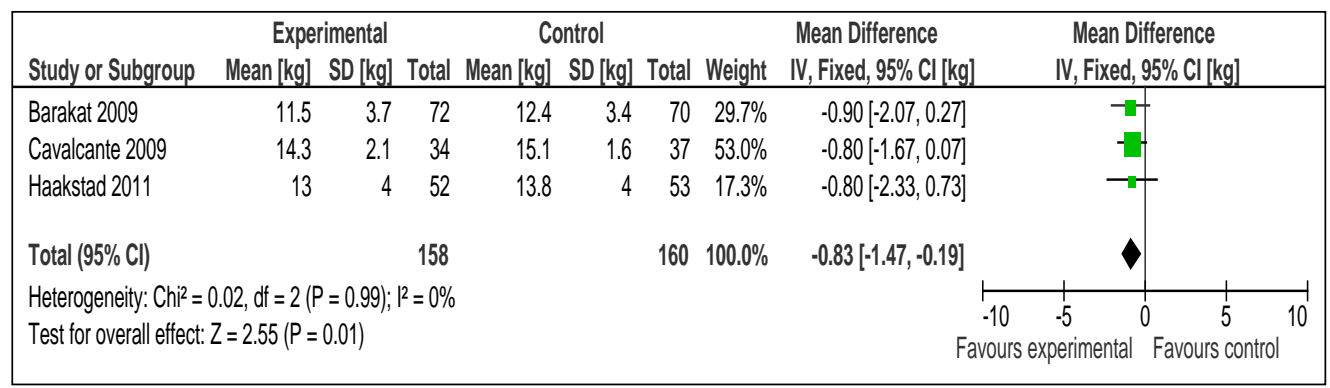

Table 2.5 Result of the "multiple content for all" studies

\begin{tabular}{|c|c|c|c|c|c|c|c|c|c|}
\hline Study or Subgroup & $\begin{array}{r}\text { Exper } \\
\text { Mean [kg] }\end{array}$ & $\begin{array}{l}\text { imental } \\
\text { SD [kg] }\end{array}$ & & \multicolumn{2}{|c|}{ Control } & Total & I Weight & $\begin{array}{l}\text { Mean Difference } \\
\text { IV, Fixed, } 95 \% \text { Cl [kg] }\end{array}$ & $\begin{array}{c}\text { Mean Difference } \\
\text { IV, Fixed, } 95 \% \text { Cl [kg] }\end{array}$ \\
\hline Jackson 2011 & 15.15 & 1.96 & 134 & 15.24 & 1.96 & 153 & $86.7 \%$ & $-0.09[-0.54,0.36]$ & \\
\hline Kinnunen, 2007 & 14.6 & 5.4 & 48 & 14.3 & 4.1 & 56 & $5.1 \%$ & $0.30[-1.57,2.17]$ & \\
\hline Phelan 2011 & 15 & 6.9 & 179 & 15.65 & 7.5 & 184 & $8.2 \%$ & $-0.65[-2.13,0.83]$ & 0 \\
\hline Total $(95 \% \mathrm{Cl})$ & & & 361 & & & 393 & $100.0 \%$ & $-0.12[-0.54,0.31]$ & 1 \\
\hline $\begin{array}{l}\text { Heterogeneity: Chi² = } \\
\text { Test for overall effect. }\end{array}$ & $\begin{array}{l}170, d f=2(P \\
=0.54(P=\end{array}$ & $\begin{array}{l}=0.70) ; \\
0.59)\end{array}$ & $P^{2}=0 \%$ & & & & & & $\begin{array}{cccc}-5 & 0 & 5 & 10 \\
\text { experimental } & \text { Favours control }\end{array}$ \\
\hline
\end{tabular}

Seven studies could not be included in a group, the so-called singleton studies. Among these, two studies had a significant difference in mean GWG in the intervention group. Quinlivan et al., ${ }^{87}$ reported a mean GWG difference of $-6.8 \mathrm{~kg}$ (7.0 kg (sd 5.2) in the intervention group versus $13.8 \mathrm{~kg}$ (sd 5.2) in the control group; $p<0.001$ ). They used a "four-step multidisciplinary approach" for overweight and obese women, in which they gave continuity of care, weighing and, if necessary, dietary and/or psychological support. Furthermore, in the study that used nerve stimulation to decrease nausea, GWG increased significantly in the first trimester of pregnancy. The other studies (resistance elastic band exercise, probiotics with diet versus placebo with and without diet, weighing and monitoring GWG, awareness and individual counselling on GWG, PA and diet, low fat versus low glycaemic load diet) reported no significant differences in GWG for the overall group. Table 2.6 gives an overview of GWG in the singleton studies.

Table 2.6 Primary outcomes of the high quality singleton studies

\begin{tabular}{ll}
\hline $\begin{array}{l}\text { Author, } \\
\text { year }\end{array}$ & Outcomes on GWG \\
\hline $\begin{array}{l}\text { Quinlivan et al, } \\
2011^{87}\end{array}$ & GWG: 7.0 se 0.65 vs 13.8 se $0.67 p<0.001$ \\
\hline $\begin{array}{l}\text { de Barros et al, } \\
2010^{89}\end{array}$ & GWG: 12.64 sd 5.29 vs 11.28 sd $5.63 p 0.324$ \\
\hline llmonen et al, & GWG: 14.8 sd 4.4 vs 14.7 sd 5.0 vs 14.8 sd $5.2 p=0.981$ \\
\hline
\end{tabular}




\begin{tabular}{|c|c|}
\hline $2010^{94}$ & exceeding IOM 1990: $50.7 \%$ vs $60.3 \%$ vs $68.6 \% p=0.1$ \\
\hline $\begin{array}{l}\text { Jeffries et al, } \\
2009^{101}\end{array}$ & $\begin{array}{l}\text { GWG: } 10.7 \text { sd } 4.21 \text { vs } 11.5 \text { sd } 4.03 \mathrm{~kg} \\
\text { exceeding IOM } 1990: 18 \% \text { vs } 23 \% \text { p } 0.42 \\
\text { GWG rate: } 0.44 \text { sd } 0.17 \text { vs } 0.46 \text { sd } 0.16 \mathrm{~kg} / \text { week } \mathrm{Cl}-0.02-0.07 \\
\text { In OW group: } 0.42 \text { sd } 0.15 \text { vs } 0.54 \text { sd } 0.12 \mathrm{~kg} / \text { wk difference } 0.12 \mathrm{Cl} 0.03-0.22 \mathrm{p} 0.01\end{array}$ \\
\hline $\begin{array}{l}\text { Luoto et al, } \\
2011^{95}\end{array}$ & GWG: 13.8 sd 5.8 vs 14.2 sd 5.1 \\
\hline $\begin{array}{l}\text { Rhodes et al, } \\
2010^{97}\end{array}$ & GWG: 6.9 sd 4.2 vs 6.4 sd 4.5 \\
\hline $\begin{array}{l}\text { Rosen et al, } \\
2003^{98}\end{array}$ & $\begin{array}{l}\text { GWG: } 1.3 \text { sd } 2.1 \text { vs } 0.5 \text { vs } 3.0 \mathrm{~kg} \mathrm{p}=0.003 \\
\text { difference }+0,8 \mathrm{~kg}\end{array}$ \\
\hline
\end{tabular}

\subsubsection{IOM-guidelines}

Four studies provided data on the number of women with GWG related to the 1990 IOMguidelines. ${ }^{86,101,102,104}$ None of these four studies reported differences in the intervention group compared to the control group. One of the studies only reported "overgain" and "not overgain"; therefore this study has no data on the percentage of women with GWG within the guidelines. ${ }^{101}$ Results are shown in Table 2.7.

Table 2. 7 Gestational weight gain in relation to Institute Of Medicine (IOM)-guidelines

\begin{tabular}{|c|c|c|c|c|}
\hline \multirow[t]{2}{*}{$\begin{array}{l}\text { Author, year, } \\
\text { intervention }\end{array}$} & \multirow[t]{2}{*}{$\begin{array}{l}\text { Sub } \\
\text { categories }\end{array}$} & \multicolumn{2}{|c|}{$\begin{array}{l}\text { Amount of women (\%) gaining weight below (<), } \\
\text { within (=), not within ( }(\neq) \text { or above }(>) 1990 \text { IOM- } \\
\text { guidelines }\end{array}$} & \multirow[t]{2}{*}{ p-value/OR } \\
\hline & & Intervention group & Control group & \\
\hline \multirow{2}{*}{$\begin{array}{l}\text { Haakstad \& B } \varnothing, \\
2011^{102}\end{array}$} & & $\neq 17 / 52$ (32.7) & $\neq 20 / 53$ (37.7) & 0.59 \\
\hline & & $=35 / 52(67.3)$ & $=33 / 53(62.3)$ & \\
\hline \multicolumn{5}{|l|}{ PA for all } \\
\hline \multirow{2}{*}{$\begin{array}{l}\text { Jeffries et al., } 2009 \\
101\end{array}$} & UW & $>0 / 5(0)$ & $>0 / 5(0)$ & not reported \\
\hline & NW & $>7 / 75(9.3)$ & $>11 / 65(16.4)$ & 0.22 \\
\hline \multirow{3}{*}{$\begin{array}{l}\text { weighing and } \\
\text { monitoring GWG } \\
\text { for all pre-BMI }\end{array}$} & OW & $>7 / 20(35)$ & $>10 / 18(55.6)$ & 0.33 \\
\hline & $\mathrm{OB}$ & $>9 / 25(36)$ & $>5 / 2123.8$ & 0.33 \\
\hline & all & $>23 / 125$ (18.4) & $>26 / 111(23.4)$ & 0.42 \\
\hline \multirow{2}{*}{$\begin{array}{l}\text { Kinnunen et al., } \\
2007^{104}\end{array}$} & & $<16 / 48(33.3)$ & $<15 / 56(26.8)$ & adjusted OR for \\
\hline & & $=10 / 48(20.8)$ & $=24 / 56(42.9)$ & exceeding IOM \\
\hline $\begin{array}{l}\text { multiple-content } \\
\text { for all }\end{array}$ & & $>22 / 48(45.8)$ & $>17 / 56(30.3)$ & $\begin{array}{l}1.82 \text { (95\% Cl 0.65- } \\
5.14)\end{array}$ \\
\hline \multirow{2}{*}{$\begin{array}{l}\text { Phelan et al., } 2011 \\
86\end{array}$} & NW & $<37 / 92(40.2)$ & $<49 / 94(52.1)$ & not reported \\
\hline & & $=42 / 92(45.7)$ & $=33 / 94(35.1)$ & \\
\hline \multirow{4}{*}{$\begin{array}{l}\text { multiple-content } \\
\text { for all }\end{array}$} & & > 13/92 (14.1) & $>12 / 94(12.8)$ & \\
\hline & $\mathrm{OW} / \mathrm{OB}$ & $<58 / 87(66.7)$ & $<55 / 90(61.1)$ & not reported \\
\hline & & $=18 / 87$ (20.7) & $=22 / 90(24.4)$ & \\
\hline & & $>11 / 87$ (12.6) & $>12 / 90(13.3)$ & \\
\hline
\end{tabular}

Abbreviations: $\mathrm{BMI}$, body mass index; $\mathrm{Cl}$, confidence interval; $\mathrm{GWG}$, gestational weight gain; IOM, Institute Of Medicine; NW=Normal weight; $\mathrm{OB}=$ Obese; $\mathrm{IOM}, \mathrm{OR}$, Odds ratio; $\mathrm{OW}=$ Overweight; $\mathrm{PA}$, physical activity; UW=Underweight; 


\subsubsection{Secondary outcomes}

Fifteen studies reported secondary outcomes, which are outcomes other than GWG or the percentage of women gaining weight within the guidelines. These are discussed in three categories: changed behaviour (PA and diet), pregnancy-related aspects (PIH and GDM) and outcomes of birth, for child and mother. Figure 2.2 (at the end of this thesis) gives an overview of the effects of the secondary outcomes. To prevent confusion it is noteworthy in this figure that diet and PA are outcomes here (not an intervention). More details of secondary outcomes are available on request with the author.

\section{Changed behaviour: physical activity and dietary behaviour}

PA change was reported in four studies (probiotics study, ${ }^{94}$ two multiple-content interventions for all pre-BMI ${ }^{84,104}$ and one multiple-content intervention for $\mathrm{GDM}^{95}$ ); none of the four interventions had significant effects on PA.

All studies that measured dietary behaviour (eight out of fifteen) reported significant changes in this behaviour. The probiotics study compared three groups: probiotic with diet, placebo with diet and placebo without diet. In this study, the two groups with diet showed a lower intake of saturated fat one year after birth. ${ }^{94}$ Two multiple-content interventions for all BMI groups $^{84,104}$ reported a healthier diet at the end of the intervention, while the study that compared low fat diet (LFD) with low glycaemic load diet (LGLD), reported that subjects in the LGLD group had more fibre in their diets and a lower glycaemic index at the end of the intervention. ${ }^{97}$ Luoto's multiple-content intervention for GDM improved the balance between saturated and unsaturated fat intake and fibre intake; the total amount of energy, carbohydrates, fat and protein did not change. ${ }^{95}$ Finally, in one of the "diet for obese" studies dietary behaviour was measured at 36 weeks of pregnancy and found a decrease in energy, protein, fat and carbohydrates intake. ${ }^{99}$ The other "diet for obese" study did not report a dietary behaviour change. ${ }^{103}$ Although it is not a dietary behaviour, nerve stimulation for first trimester pregnant women with nausea resulted in less dehydration in the intervention group. $^{98}$

\section{Pregnancy related diseases: hypertension and diabetes}

Seven out of fifteen studies reported on PIH. The two studies in the "diet for obese" group both reported a reduction of PIH. In one of these two studies $(n=232)$ hypertension was $2.6 \%$ in the intervention group and $8.6 \%$ in the control group $(p=0.05) .{ }^{103}$ The other study $(n=50)$ reported $4 \% \mathrm{PIH}$ in the intervention group versus $15 \%$ in the control group, while preeclampsia was $0 \%$ versus $4 \%$ ( $p$-value not reported)..$^{99}$ The remaining five studies found no differences in $\mathrm{PIH}^{86,87,91,95,101}$

Eight studies reported data on the treatment of GDM and glucose levels. The four-step multidisciplinary approach for overweight and obese women led to a reduction of GDM (6\% versus $29 \% ; p=0.04) .{ }^{87}$ Women with GDM who exercised with an elastic band showed a significant decrease in the amount of required insulin $(21.9 \%$ versus $56.3 \% ; p=0.01)$ and an increase in time spent within the target glucose range (63\% versus $41 \% p=0.01) .{ }^{89}$ In the 
probiotics study, the group with probiotic and diet showed a decrease in glucose levels at six and twelve months after birth compared with the groups that had placebo with diet and placebo without a diet. ${ }^{94}$ Five studies showed no significant differences in GDM. ${ }^{86,95,99,101,103}$

\section{Obstetric outcomes: birth, child and mother}

Nine studies reported gestational age at birth; none of them showed a significant difference between the intervention group and the control group. ${ }^{86,89-91,94,97,99,101,103}$ Six studies reported vaginal birth rates but none of them reported significant differences. ${ }^{86,91,97,99,101,103}$ Water aerobics, part of one of the studies in the "PA for all" group $(n=71)$, diminished the number of women requiring analgesia during birth $(27.3 \%$ versus $64.9 \% \mathrm{p}=0.00) .{ }^{91}$

The child's birth-weight was reported in nine out of fifteen studies $86,87,91,94,95,99,101,103,104$ and differed in two studies. ${ }^{95,104}$ A multiple-content intervention for all pre-pregnancy BMI groups reported a decrease in children weighing above 4000 grams, no difference was found in children below 2500 grams. ${ }^{104}$ A multiple-content intervention for GDM women resulted in a lower mean birth weight of 133 grams $(p=0.04)$ and a lower percentage of lga children $(12.1 \%$ versus $19.7 \%$; $p=0.04) .{ }^{95}$ Abdominal circumference of the baby - a secondary outcome of the intervention where low fat diet was compared with low glycaemic load diet - did not differ. ${ }^{97}$ This study reported a significant effect on baby's head circumference: the LGLD group had bigger head circumferences than the LFD group $(35.0 \mathrm{~cm}$ versus $34.2 \mathrm{~cm} ; \mathrm{p}=0.01) .{ }^{97}$ Three other studies also reported head circumference, but showed no significant differences. ${ }^{94,95,99}$ Length of the child was reported in three studies, ${ }^{94,95,99}$ low Apgar score in two studies, ${ }^{101,103}$ hypoglycaemia in one study, ${ }^{101}$ shoulder dystocia in one study ${ }^{101}$ and placental weight in one study, ${ }^{99}$ all without significant differences.

Mother-related outcomes were reported in eight of the fifteen studies. ${ }^{84,86,87,91,94,95,98,101}$ The nerve stimulation for women with nausea had no effect on the rate of ketonuria or symptoms of nausea, vomiting and retching. ${ }^{98}$ One study reported a significant difference in post-partum waist circumference. ${ }^{94}$ However, the baseline weight of the intervention group was significantly lower than in the control group, which might explain the difference. Phelan et al. provided a multiple-content intervention for all pre-pregnancy BMI groups and measured women's weight six months after birth. ${ }^{86}$ In the intervention group they found a higher rate of women who had a weight below their pre-pregnancy weight compared to the control group (in women with normal weight $35.6 \%$ versus $20.7 \%$, and in overweight and obese women $25.6 \%$ versus $16.7 \%) .{ }^{86}$ Water aerobics resulted in a lower body fat and fat free mass at the end of pregnancy. ${ }^{91}$

The study that compared a LFD with an LGLD measured a variety of blood parameters and showed significant differences in C-reactive protein (CRP) (LGLD decreased more than LFD). ${ }^{97}$ However, the CRP at baseline was significantly higher in the LGLD group. Furthermore, this study reported a difference in the increase of total cholesterol and triglycerides (for both parameters LGLD showed a lower increase than LFD). ${ }^{97}$

Quinlivan et al. measured cholesterol levels after the four-step multidisciplinary approach but did not find any differences; ${ }^{87}$ they also found no differences when measuring other blood 
parameters, fat free mass and skin folds. The study of Haakstad and B $\emptyset$, one of the "PA for all" group, also measured skin folds, and found no differences between the intervention group and the control group. ${ }^{102}$ One of the "diet for obese" studies reported significant differences in the decrease of leptin concentration (-39 versus $-8 ; p=0.00) .{ }^{99}$ Jackson et al. found that their multiple-content intervention for all pre-pregnancy BMI groups increased knowledge levels about adequate GWG and more discussions with health care providers about weight, nutrition or exercise. ${ }^{84}$

\subsection{Discussion}

The 28 intervention studies showed a great range in types of interventions and target groups, suggesting that the problem of GWG is complex and widespread and can be approached through various pathways. Because the interventions we studied often included several elements it was not possible to identify the exact features of the intervention that were effective. While some studies did use behavioural theories to underpin their intervention, none was based on a systematic approach to the problem (e.g., Intervention Mapping). ${ }^{62}$ As a second step, we assessed the methodological quality of the completed studies. Fifteen of the 28 studies were of high quality; we described the primary and secondary outcomes of these studies.

Ronnberg and Nilsson earlier reviewed intervention studies using the GRADE approach for quality assessment. ${ }^{78}$ They did not perform a meta-analysis because of the poor quality of the studies included. They excluded two of the studies, which we included in our analysis. ${ }^{99,104}$ They downgraded the RCT of Wolff et al. because the researchers did not describe their concealment of allocation clearly and because they had a high loss to follow up. In our study however, Wolff et al. met our quality criterion of 7 points on the PEDro scale. Ronnberg and Nilsson did not upgrade the study of Kinnunen et al., ${ }^{78,104}$ because of a lack of clarity about how participants were informed and because of unexplained losses to follow up. We upgraded this study twice, because we found an absence of confounders and we expected the effect of the intervention to be larger than found. Furthermore, we found that participants were well informed. Therefore Kinnunen et al. was included in our meta-analysis. If we were to leave this study out of the meta-analysis however, it would not change our findings.

In their review Streuling et al. included RCT's that we did not find. ${ }^{75}$ They used other keywords (for instance "pregnancy outcome" was added with "weight gain") and more databases (BIOSIS Previews, Journal Citation Reports, and ISI Web of Knowledge), and found three studies with GWG as a secondary outcome, and four studies that did not mention GWG in the title. ${ }^{75}$ Because we only searched for interventions with GWG as primary outcome, we missed these studies. However, their conclusion - that physical activity leads to a reduction of GWG of almost one kilogram - was similar to ours. 
In spite of the differences between our review and the earlier reviews of Streuling et al. and Ronnberg and Nilsson, ${ }^{75,78}$ we are confident that our work provides a thorough and accurate picture of what we know about interventions for GWG.

In GWG research, it is seldom possible to measure weight at the beginning of a pregnancy, because most women start their pregnancy check-ups when they have been pregnant for six weeks or more. Some researchers choose to use the measured weight at the start of pregnancy check-ups, while others use the self-reported weight of women before pregnancy. It is therefore difficult to draw conclusions about the actual weight gained in pregnancy. However, differences in GWG in the intervention and control groups can be compared, as long as the weighing method is similar and consistently used in both groups.

In non-pregnant women, being over-weight is associated with psychological factors, ${ }^{114}$ so we expected to find some interventions aimed at this component. However, only Quinlivan's four step multidisciplinary intervention used a psychologist, and then only if necessary. ${ }^{87}$ This omission was also mentioned by Skouteris and Walker. ${ }^{47,71}$

It is also striking that we found no intervention for increasing awareness among care providers. This is surprising because we know that there is a relationship between GWG during pregnancy and the recommendations of care providers. ${ }^{115}$ We also know that not all women receive proper advice from their care providers. ${ }^{115}$ It could be that part of the problem of unhealthy GWG will be diminished when health care providers are made aware of the IOM-guidelines and the importance of meeting them.

\subsubsection{Primary outcome: GWG or percentage of women gaining within the guidelines?}

The percentage of women gaining within the guidelines did not differ in any of the four studies that examined GWG in relation to the IOM-guidelines.

The meta-analyses showed that GWG can be reduced in obese women by prescribing a diet and helping them to stick to the diet. Furthermore the analyses showed that PA has limited effects in reducing GWG. The intervention studies demonstrating differences in GWG did not measure percentages of women with GWG within the IOM-guidelines. We do not know whether the women who needed to gain less weight did in fact gain less weight, which is a missed opportunity. However, it is useful to know which interventions can be effective for obese women. The four-step multidisciplinary approach ${ }^{87}$ looks promising and needs to be studied again for larger groups, different populations and relationship to IOM-guidelines. For women who lose weight during the first trimester because of nausea and vomiting, a nerve wrist band might be helpful, this intervention needs further study to prove its effect.

\subsubsection{Secondary outcomes and the complexity of GWG}

Secondary outcomes in these studies were not entered in a meta-analysis. Several benefits were reported, varying from requiring less analgesia in labour and less need for insulin among women with GDM to better dietary behaviour. The interventions deployed in these studies 
had a further reach than simply establishing a healthy GWG. Finally, we can also conclude that there are no indications that any of the interventions were harmful.

Morisset et al. studied interventions for GDM, GWG and pre-pregnancy BMI and introduced an interesting theory about the relationship between these three issues: they believed it is important to monitor glucose levels beginning in early pregnancy. ${ }^{68}$ They suggested treating overweight and obese women early in pregnancy in order to limit excessive GWG, maintain euglycaemia and reduce the risk of macrosomia. Guelinckx et al. found that women whose BMI increased from 23 to $25 \mathrm{~kg} / \mathrm{m}^{2}$ after their first pregnancy doubled their risk of GDM in their next pregnancy. ${ }^{116}$

\subsubsection{Comments on the review methodology}

This is the first systematic review of all interventions targeting GWG in all BMI-groups. We were able to identify three clinically homogenic groups and perform a meta-analysis, which provided a number of new and practical insights about interventions for achieving a healthy GWG. However, new RCT's on GWG have been published, after we started this review and ended our search. ${ }^{117-119}$ We look forward to learn what these new studies will add to our body of knowledge.

Adherence is an important predictor of healthy GWG. ${ }^{103,120}$ Women who are adherent differ in psychological or physiological characteristics that are as yet unexplored. Knowing these characteristics may provide useful ideas about how to use interventions in subgroups. ${ }^{103}$ Details of the content of the interventions included in this review, the rationale behind it, and the different parts of the interventions were often lacking. Many words are needed to describe an intervention, which might result in an author prioritising the methods and results sections of their study. We did not contact authors for further information.

Although we were interested in the interventions' programme elements, we did not study behavioural aspects. Gardner et al. examined behavioural goals in the intervention they studied: their review can be seen as complementary to our review. ${ }^{63}$ Our review considered only prenatal interventions: we did not include interventions after birth. This topic is worthy of additional investigation because weight retention of the mother in the first year after giving birth is related to subsequent over-weight. ${ }^{20}$ Because we found published studies where interventions had no measureable effects, we chose not to measure publication bias. Streuling et al. did measure publication bias and found no reason to believe that this kind of bias was present in studies of this type. ${ }^{75}$

\subsection{Conclusion}

We found no studies that described interventions that increased the number of women with GWG within the IOM-guidelines. We did find, however, that GWG was successfully reduced for obese women when they followed a diet and had support to stick to the diet.

Furthermore, we found that physical activity helped to decrease GWG. Interventions aimed at achieving a healthy GWG provide ancillary and positive effects. The challenge remains to find an intervention that offers tools for health professionals to help women achieving a GWG 
within the IOM-guidelines. More research is needed, on the determinants that drive both healthy and unhealthy GWG, and on interventions that can help women achieve a healthy GWG. Within this research, GWG needs to be related to the IOM-guidelines.

\subsection{Acknowledgement}

Sanne Kohl provided help with analysing the ongoing studies. The language support Centre of Maastricht University provided editorial assistance. 


\title{
Chapter 3
}

\section{Weight gain in healthy pregnant women in relation to pre-pregnancy BMI, diet and physical activity}

\author{
Astrid Merkx, Marlein Ausems, Luc Budé, Raymond de Vries, \\ Marianne J. Nieuwenhuijze
}

Midwifery 2015 Jul; 31(7):693-701 


\section{Objective}

To explore gestational weight gain in healthy women in relation to pre-pregnancy Body Mass Index, diet and physical activity.

\section{Design}

A cross-sectional survey was conducted among 455 healthy pregnant women of all gestational ages receiving prenatal care from an independent midwife in the Netherlands. Weight gain was assessed using the Institute of Medicine (IOM) guidelines and classified as below, within, or above the guidelines. A multinomial regression analysis was performed with weight gain classifications as the dependent variable (within IOM-guidelines as reference). Independent variables were pre-pregnancy Body Mass Index (BMI), diet (broken down into consumption of vegetables, fruit and fish) and physical activity (motivation to engage in physical activity, pre-pregnancy physical activity and decline in physical activity during pregnancy). Covariates were age, gestational age, parity, ethnicity, family income, education, perceived sleep deprivation, satisfaction with pre-pregnancy weight, estimated prepregnancy body mass index, smoking, having a weight gain goal and having received weight gain advice from the midwife.

\section{Findings}

Forty-two percent of the women surveyed gained weight within the guidelines, $14 \%$ of the women gained weight below the guidelines and $44 \%$ gained weight above the guidelines. Weight gain within the guidelines, compared to both above and below the guidelines, was not associated with pre-pregnancy BMI nor with diet. A decline in physical activity was associated with weight gain above the guidelines (OR $0.54,95 \% \mathrm{Cl} 0.33-0.89$ ). Weight gain below the guidelines was seen more often in women who perceived a greater sleep deprivation (OR $1.20,95 \% \mathrm{Cl} 1.02-1.41)$. Weight gain above the guidelines was seen less often in Caucasian women in comparison to non-Caucasian women (OR 0.22, 95\% $\mathrm{Cl} 0.08-0.56$ ) and with women who did not stop smoking during pregnancy (OR 0.49, 95\% $\mathrm{Cl} 0.25-0.95$ ).

\section{Key conclusions and implications for practice}

A decline in physical activity was the only modifiable factor in our population associated with weight gain above the gain recommended by the guidelines. Prevention of reduced physical activity during pregnancy seems a promising approach to promoting healthy weight gain. Interventions to promote healthy weight gain should focus on all pregnant women, regardless of pre-pregnancy BMI. 


\subsection{Introduction}

Gestational weight gain (GWG) is associated with the health of mothers and babies. ${ }^{22}$ Too much GWG is associated with being overweight and obese in the long term in both mothers and their offspring. ${ }^{3,19,22,121}$ Too much GWG is also associated with pregnancy-related pathology, including gestational diabetes and pregnancy-induced hypertension. ${ }^{22,122}$ Too little GWG is associated with prematurity and babies who are small for their gestational age. ${ }^{122,123}$ Given the need to prevent pathologies caused by unhealthy GWG, it is critical that professionals in prenatal care stimulate healthy GWG.

Recommendations for healthy GWG are described in the Institute of Medicine (IOM)guidelines for GWG. ${ }^{6}$ These American guidelines are used often in research around the globe, but have been adopted by only a small (albeit growing) number of national professional boards. ${ }^{124}$ The IOM recommends a total GWG that is dependent on the Body Mass Index (BMI) before pregnancy (Table 3.1). ${ }^{6}$

Table 3.1 Institute of Medicine (IOM)-guidelines for gestational weight gain (GWG)

\begin{tabular}{lcccc}
\hline classification & $\begin{array}{l}\text { Body Mass Index } \\
\text { before pregnancy in } \\
\mathbf{k g} / \mathbf{m}^{\mathbf{2}}\end{array}$ & $\begin{array}{l}\text { GWG range in kg } \\
\text { for course of } \\
\text { pregnancy }\end{array}$ & $\begin{array}{l}\text { GWG range in } \\
\text { kg for first 13 } \\
\text { weeks }\end{array}$ & $\begin{array}{l}\text { GWG range in kg per week } \\
\text { in second and third } \\
\text { trimester }\end{array}$ \\
\hline underweight & $<18.5$ & $12.5-18$ & $0.5-2$ & $0.44-0.58$ \\
\hline normal weight & $18.5-24.9$ & $11.5-16$ & $0.5-2$ & $0.35-0.50$ \\
\hline overweight & $25.0-29.9$ & $7-11.5$ & $0.5-2$ & $0.23-0.33$ \\
\hline obese & $\geq 30.0$ & $5-9$ & $0.5-2$ & $0.17-0.27$ \\
\hline
\end{tabular}

Abbreviations: GWG, Gestational Weight Gain; IOM, Institute of Medicine

For all pre-pregnancy BMI groups (underweight, normal weight, overweight and obese), the recommendation for healthy GWG is the same for the first 13 weeks, from 0.5 to 2.0 kilograms. In the second and third trimesters of pregnancy the guidelines recommend a maximum and minimum rate of GWG per week, depending on the pre-pregnancy BMI. ${ }^{6}$ IOMguidelines enable and encourage monitoring GWG from the beginning to the end of pregnancy. Given the earlier mentioned negative consequences of unhealthy GWG, and because women with too high GWG in the first part of their pregnancy have a higher risk of exceeding the guidelines in the end, ${ }^{45,123,125}$ monitoring GWG during pregnancy is important. Supportive health professionals should strive to help women reach a healthy GWG during pregnancy, i.e. a GWG within the IOM-guidelines.

Various studies of different populations of women from western countries report that the number of women achieving a healthy GWG varies between 21.6 to $48.7 \%$. ${ }^{41,126}$ We know from the literature that two behaviours are closely related to healthy GWG: healthy diet and engaging in physical activity (PA). ${ }^{6,43}$ It is often recommended that pregnant women avoid food that can harm their baby through toxoplasmosis and listeriosis. These recommendations are not related to the promotion of healthy GWG. Non-pregnant, healthy individuals are 
advised to eat 200 grams of vegetables per day, two to three daily servings of fruit, fish twice per week (including fatty fish once a week) and to adapt their caloric intake to their individual PA. ${ }^{127}$ Norms for healthy PA are 30 minutes of moderate-intensive PA at least five times per week. ${ }^{128}$ The norms for diet and PA for healthy non-pregnant individuals are applicable to pregnant women (except for women with a medical reason for adapting their lifestyle). A large number of non-pregnant individuals do not meet the norms for diet and PA. ${ }^{129,130}$ Little is known about pregnant women's eating and PA behaviour.

GWG is also associated with several factors other than diet and PA. Women with healthy GWG are more often Caucasian, ${ }^{41,48}$ are better educated about GWG, ${ }^{86}$ do not smoke, ${ }^{123,131,}$ 132 are multiparous, ${ }^{133}$ are more likely to have a healthy GWG goal ${ }^{59,60}$ and exhibit more positive self-esteem. ${ }^{43,44,134,135}$ Women with too much GWG, in contrast, more often report sleep deprivation, ${ }^{44,136,137}$ have a history of restrained eating, food insecurity, low income, ${ }^{138-}$ ${ }^{140}$ a high pre-pregnancy BMI ${ }^{44,141}$ and too much GWG in the first part of pregnancy. ${ }^{45,125,142}$ They are more likely to receive inaccurate (too high) GWG advice from their health professional $^{86}$ and more often have disturbed blood parameters, such as thyroid hormones. ${ }^{36}$ The above-mentioned studies mostly included specific subgroups such as obese women or women with a higher risk for gestational diabetes. At present we know very little about how these factors influence GWG in interaction with each other and with pre-pregnancy BMI, diet and PA in healthy pregnant women. We assume that at least $52 \%$ of pregnant women in the Netherlands are healthy, as these women receive full prenatal care from a midwife which in the Dutch midwifery system means that there are no signs of pathology. ${ }^{143}$

Preventing unhealthy GWG is challenging. Behavioural interventions, including motivational interviewing, goal setting and tailored counselling, appear to be promising strategies for influencing diet and the PA of pregnant women with specific conditions such as diabetes or over-weight. ${ }^{22,43,144-146}$ Research and interventions aiming at healthy pregnant women are scarce, however, despite the fact that two thirds of this group have unhealthy GWG. ${ }^{45}$ Efforts to increase our knowledge of the physiology of GWG will benefit from studies of the GWG distribution of healthy pregnant women and from analysis of the factors associated with (un)healthy GWG in healthy women. This knowledge is a prerequisite for developing an effective intervention for promoting healthy GWG in all pregnant women. ${ }^{62}$

\subsection{Methods}

We used an explorative cross-sectional survey design with a sample of healthy pregnant women of all gestational ages.

\subsubsection{Procedure}

Midwives working in midwifery-led community practices are the main care providers in the Netherlands for healthy pregnant women. ${ }^{143}$ Midwifery-led community practices ( $n=140$ ) affiliated with our institute (for clinical placements of our students) were invited by email to participate in the study. A total of 30 practices agreed. These practices were spread over the southern part of the Netherlands and were located in urban, semi-urban and rural areas. 
From September to November 2012 the midwives working in these practices offered information packages to pregnant women visiting their prenatal care clinics. The packages included study information, an informed consent form and a stamped reply envelope. Women who were interested in participating in the study were asked to authorize their midwife to provide their name, phone number and time preference for a phone call from the research team. The researcher (first author) subsequently telephoned the interested woman and explained the research aim and study procedures. Women who agreed to participate were asked to return a completed consent form. Depending on the preference of the participant, a study questionnaire was then sent by email with a link to the digital questionnaire, as a printed version by regular mail or as an interview by phone by a trained researcher. Reminders (for the email preference only) were sent after one week. As an incentive to improve the response rate, participants were invited to take part in a raffle of a skincare package. To preserve confidentiality all data was stored in a protected file. This study is part of Promoting Healthy Pregnancy, a research project aiming to develop an intervention to increase the number of women achieving healthy weight gain during their pregnancy. The Research Ethics Committee of Atrium-Orbis-Zuyd reviewed the study protocol and confirmed that ethical approval was not required.

\subsubsection{Measures}

Our questionnaire included the following items:

Demographics and characteristics: including the items date of birth, current care provider (midwife/obstetrician), parity $(0,1, \ldots)$, country of birth (of participant's mother, father and self), family income (above or below median household income), ${ }^{147}$ education level (highest diploma) and date of expected birth. We also asked about sleeping behaviour (hours per day), perceived sleep deprivation in the last week (number of days per week) and smoking behaviour (smoking behaviour before pregnancy, change during pregnancy and amount smoked).

Weight and weight gain: We asked for self-reported pre-pregnancy weight (kg), self-reported height $(\mathrm{m})$, date of last weighing and weight at that time $(\mathrm{kg})$. We asked estimated prepregnancy BMI category (underweight, normal weight, overweight, obese), the presence of a weight gain goal and if so, what the goal was, whether or not the midwife advised a GWG goal and the degree of satisfaction with past body weight (1=very dissatisfied, $7=$ very satisfied).

Diet: The Food Frequency Questionnaire (FFQ) Vegetables, validated for pregnant women, ${ }^{148}$ was used to measure vegetable consumption. Items included the number of days of the week vegetables were eaten in three different forms: cooked vegetables during meals, raw vegetables during meals and raw vegetables as snacks. We also asked about the average amount of vegetables eaten per day. The original FFQ measures vegetable consumption in terms of 'vegetable spoons'. Because participants in the pre-test experienced difficulties assessing the amount of a 'vegetable spoon', we substituted spoons with photos of plates representing varying amounts of vegetables (Figure 3.1). ${ }^{149}$ Included were six photos of plates with string beans, varying from 30 to 250 grams, six photos of plates with lettuce, varying 
from 15 to 75 grams and five photos of plates with cucumber, varying from 25 to 150 grams. Women were asked to select a photo representative of their vegetable consumption in the last week. We used two items for fruit consumption (how many days did you eat fruit last week and how many servings of fruit did you eat on such a day) and two for fish consumption (how many times did you eat fish last week, and did you eat fatty fish at least one of those times, with the names of the nine most consumed fatty fishes).

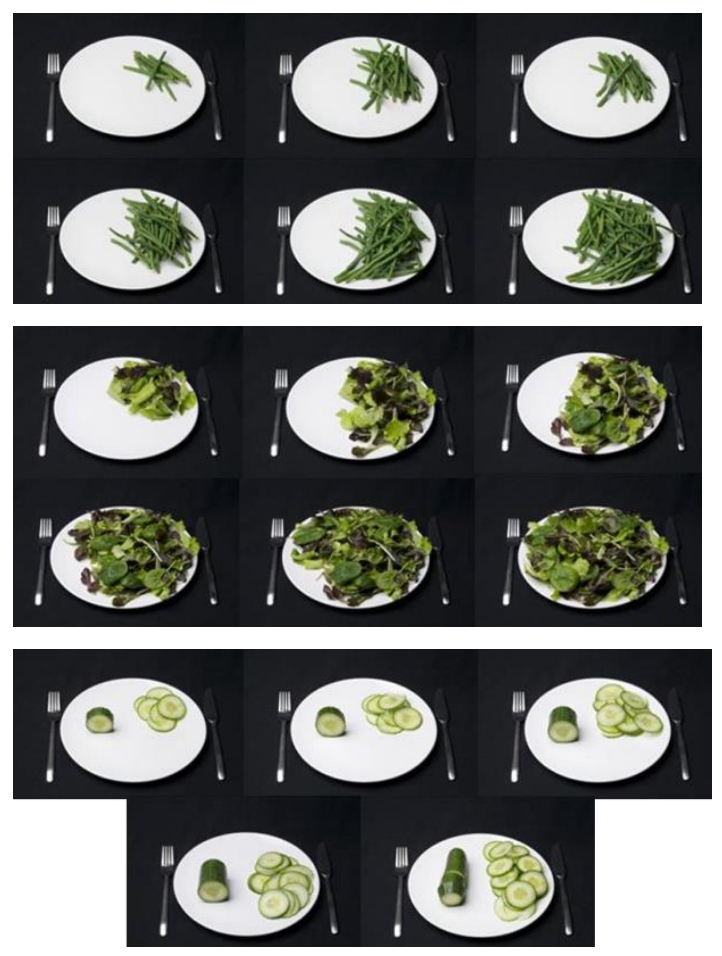

Figure 3.1 Plates of vegetables

Physical Activity (PA): Since existing questionnaires for PA were either too long ${ }^{150}$ or not validated for pregnancy, ${ }^{151}$ and the use of pedometers is not recommended for pregnant women, ${ }^{152}$ we asked the participants how many minutes in the last week they walked, cycled, engaged in sports, undertook short rigorous activities or worked in the home and garden. We asked women to respond from 1 (totally disagree) to 7 (totally agree) to the statement "I want to have healthy PA during pregnancy". Finally we asked women to rate their prepregnancy activity ( $1=$ not active at all, $7=$ very active) and whether or not they reduced their PA during pregnancy.

\subsubsection{Analyses}

Raw data was cleaned and checked for normality. The scales for time spent on various types of activity were not normally distributed and were therefore not used. "Hours of sleep per 
day" was removed because it had more than $10 \%$ missing values. For the item of GWG-goal in kilos, we observed answers indicating that women were not sure whether we were interested in total GWG-goal, bodyweight-goal at the end of pregnancy or bodyweight-goal after birth. Therefore we were not able to calculate whether a GWG-goal, if any, was adequate or not. Consequently GWG-goal became a dichotomous variable, expressing whether or not women had set a weight gain goal.

\section{Dependent variable}

In order to determine a healthy GWG, we used the self-reported weight gained at the last day of weighing in pregnancy, compared to the self-reported pre-pregnancy weight. GWG in the first 13 weeks of pregnancy was healthy if it was between 0.5 and $2.0 \mathrm{~kg}$. For women with a gestational age higher than 13 weeks, we compared their GWG with the lower and upper limits of GWG corresponding to the gestational age at the moment of weighing, using formulas described by others. ${ }^{153,154}$ We labelled this outcome GWG-IOM with three categories: below, within and above IOM-guidelines.

\section{Independent variables}

We used pre-pregnancy BMI (pre-pregnancy weight divided by squared height), total Vegetable consumption in grams per week, Fruit-norm (met/not met), Fish-norm (met/not met), Motivation healthy PA (1-7), Decline in PA (yes/no) and pre-pregnancy PA (1-7). Fruitnorm was met when at least two pieces of fruit were eaten daily during the past week and Fish-norm was met when fish was eaten at least twice during the last week, including at least one serving of fatty fish. Because we could not use the actual PA, we used the proxy Motivation healthy PA. According to the Self Determination Theory (SDT), ${ }^{155}$ motivation is an important determinant for behaviour. In a review concerning SDT and outcomes relating to PA, internal motivation was seen as an important determinant for actually engaging in PA. ${ }^{156}$ We also used the Decline of PA in pregnancy and Pre-pregnancy PA as a measure for PA, since Ajzen suggested that past behaviour is highly correlated with future behaviour. ${ }^{157}$

\section{Covariates}

Based on the literature we included a number of covariates in our analyses, including Age (years), Gestational age (weeks), Parity (dichotomised as nulliparous/multiparous), Ethnicity (Caucasian/non-Caucasian), Family income (low/high), Education (low for women without a high school diploma/entry-level qualification for women with a high school diploma/ high for women with higher professional education or university), Estimated pre-pregnancy BMI (underestimated/correct/overestimated) and Smoking (continued/stopped/non-smoking).

\section{Descriptives}

Means and standard deviations were calculated for all continuous variables. Frequencies were calculated for the outcome GWG-IOM and for the categorical variables. We crosstabulated pre-pregnancy $B M I$ categories with GWG-IOM. To check for selection bias, we 
compared the descriptives of the women with complete data (i.e. excluding women with missing data) with the total group of our respondents, and if possible with data from the Dutch Perinatal Registrations, ${ }^{143}$ the Dutch Central Bureau of Statistics ${ }^{158}$ and the National Institute for Public Health and the Environment. ${ }^{129}$

\section{Multinomial logistic regression}

To test whether gaining weight in accordance with the IOM-guidelines (GWG-IOM) was associated with pre-pregnancy BMI, diet and PA, we used a multinomial logistic regression with two comparisons: 1 ) women with GWG below IOM-guidelines compared to women with GWG within IOM-guidelines and 2) women with GWG above IOM-guidelines compared to women with GWG within IOM-guidelines.

Independent variables were entered: Pre-pregnancy BMI, dietary variables (Vegetable consumption, Fruit-norm, Fish-norm), PA-variables (Motivation healthy PA, Decline of PA, Prepregnancy $P A$. We corrected for the following confounders: Age, Gestational age (at time of last weighing), Parity, Ethnicity, Family-income, Education, Perceived sleep deprivation, Satisfaction with pre-pregnancy weight, Estimated pre-pregnancy BMI, Smoking, GWG-goal and GWG advice from midwife.

\subsection{Results}

In total 950 women received information about the study and 550 agreed to participate and returned a completed consent form. Ten consent forms arrived after our deadline for inclusion. 475 women filled out the questionnaire (a response rate of 50\%). Five of the 92 questionnaires we received by post arrived after the deadline. Twenty of the 475 questionnaires received in time were excluded (four received prenatal care from an obstetrician, twelve had given birth and four did not provide an expected date of birth) (Fig 3.2).

\subsubsection{Characteristics of the participants}

Pre-pregnancy BMI could not be calculated for 29 women (6.4\%) and GWG could not be calculated for an additional 27 women (5.9\%) due to missing data on last weight or date of weighing. Therefore, we had 56 subjects with missing data on GWG-IOM. Three more women had a missing value on income, BMI-perception and GWG-goal. In total 396 women had completed data for performing the analyses (87.0\%).

Table 3.2 shows the descriptives for all 455 women in the study and for the 396 women having no missing values. Differences between them are marginal. Our study sample seemed to consist of more Caucasian women and higher educated women than the reference group, and seemed to have higher intakes of vegetables and fruit, smoked less and ate less fish than the reference group. Of the analysed group, the mean pre-pregnancy BMI was $24.0 \mathrm{~kg} / \mathrm{m}^{2}$. Consumption of vegetables in the last week varied from 45 to 2600 grams, with a mean of 953 grams (sd 447). Vegetable-norm, Fruit-norm and Fish-norm were met in respectively 54 (13.6\%), 187 (47.2\%) and 27 women (6.8\%). Motivation for healthy diet and for healthy PA 
was on average respectively 6.2 (sd 1.0) and 5.6 (sd 1.2). More than half of the women $(n=216,54.5 \%)$ reported a decline in PA during pregnancy and the mean pre-pregnancy PA was moderately active (4.6 sd 1.2).

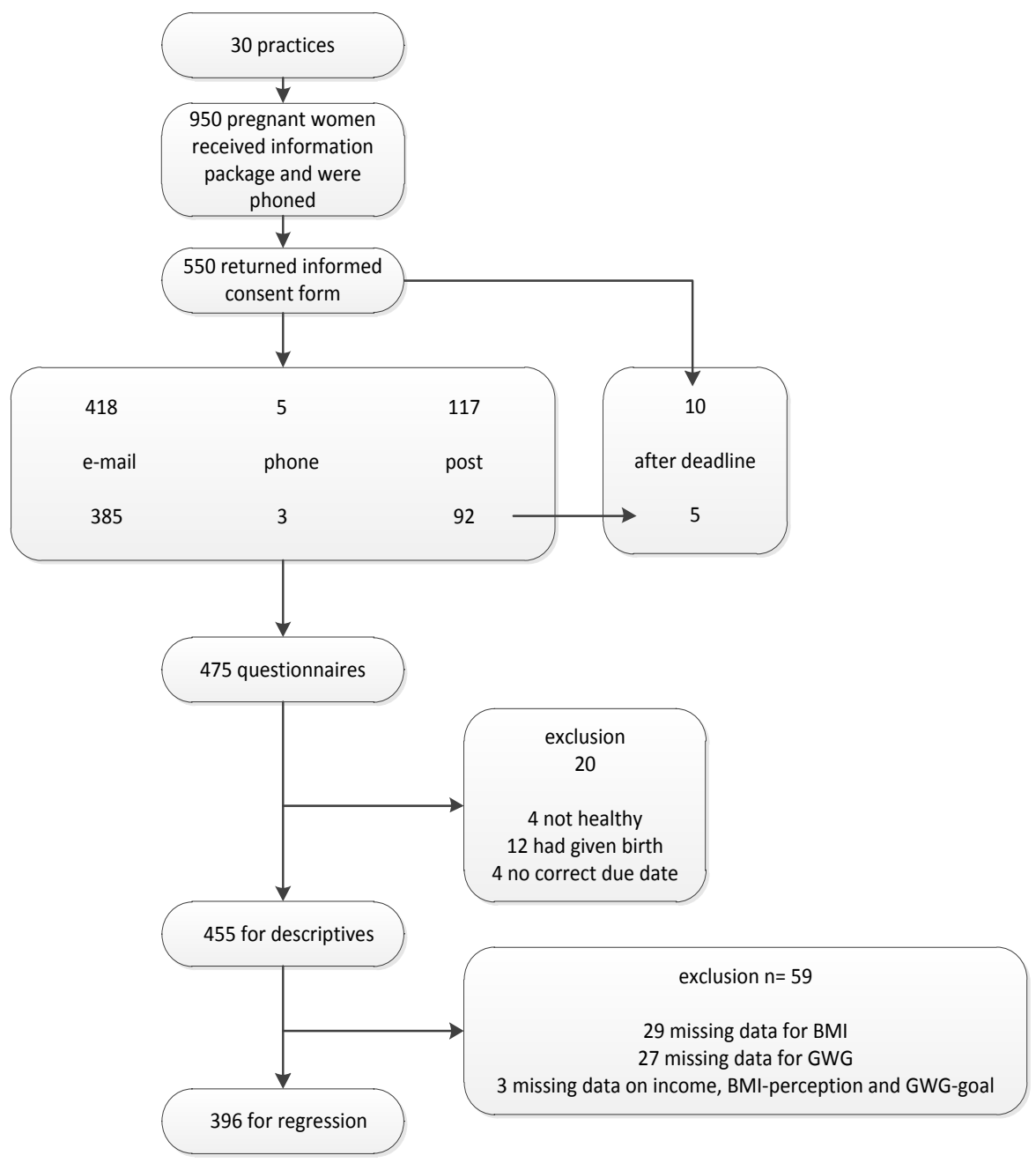

Figure 3.2 Flow diagram participants 
Table 3.2 Characteristics of participants

\begin{tabular}{|c|c|c|c|c|}
\hline & & \\
\hline & & $\begin{array}{l}\mathrm{n}=396 \text { (cases missing } \\
\text { values excluded) } \\
\text { Mean (sd) / } \mathrm{n}(\%)\end{array}$ & $\begin{array}{l}\mathrm{n}=455 \text { (cases missing } \\
\text { values included) } \\
\text { Mean (sd) / } \mathrm{n}(\%)\end{array}$ & $\begin{array}{l}\text { Reference } \\
\text { general } \\
\text { population }\end{array}$ \\
\hline age & years & $30.7(3.8)$ & $30.6(3.8)^{a}$ & $30.9(4.9)^{143}$ \\
\hline gestational age & weeks & $28.3(8.2)$ & $28.2(8.3)$ & \\
\hline $\begin{array}{l}\text { gestational age } \\
\text { at the time of } \\
\text { weighing }\end{array}$ & weeks & $26.6(8.9)$ & $26.5(9.2)^{b}$ & \\
\hline parity & nulliparous & $197(49.7)$ & $230(50.5)$ & $45 \%^{143}$ \\
\hline ethnicity & Caucasian & 363 (91.7) & $420(92.3)$ & $85.9 \%^{\mathrm{e}}$ \\
\hline family income & above modal & $294(74.2)$ & $341(75.3)^{c}$ & \\
\hline education & low & $24(6.1)$ & $30(6.6)$ & $30.9 \%^{f}$ \\
\hline & entry-level & $138(34.8)$ & $156(34.3)$ & $41.3 \%^{f}$ \\
\hline & higher education & $234(59.1)$ & $269(59.1)$ & $27.8 \%^{f}$ \\
\hline $\begin{array}{l}\text { pre-pregnancy } \\
\text { BMI }\end{array}$ & $\mathrm{kg} / \mathrm{m}^{2}$ & $24.0(4.2)$ & $24.0(4.2)^{d}$ & see Table 3.3 \\
\hline $\begin{array}{l}\text { vegetable } \\
\text { consumption }\end{array}$ & grams per week & $953(447)$ & $949(440)$ & $\begin{array}{l}\text { 95-119/day } \\
(=665- \\
833 / w k)^{129}\end{array}$ \\
\hline fruit-norm & norm is met & $187(47.2)$ & $211(46.4)$ & $7-13 \%{ }^{129}$ \\
\hline fish-norm & norm is met & $27(6.8)$ & $29(6.4)$ & $11-19 \%{ }^{129}$ \\
\hline $\begin{array}{l}\text { motivation } \\
\text { healthy eating }\end{array}$ & $\begin{array}{l}\text { 1=not motivated at } \\
\text { all, } 7=\text { very } \\
\text { motivated }\end{array}$ & $6.2(1.0)$ & $6.2(1.0)$ & \\
\hline $\begin{array}{l}\text { motivation } \\
\text { healthy PA }\end{array}$ & $\begin{array}{l}\text { 1=not motivated at } \\
\text { all, } 7=\text { very } \\
\text { motivated }\end{array}$ & $5.6(1.2)$ & $5.6(1.2)$ & \\
\hline pre-pregnancy PA & $\begin{array}{l}\text { 1=very inactive, } \\
\text { 7=very active }\end{array}$ & $4.6(1.2)$ & $4.7(1.2)$ & \\
\hline $\begin{array}{l}\text { decreased PA } \\
\text { during pregnancy }\end{array}$ & yes & $216(54.5)$ & $251(55.2)$ & \\
\hline $\begin{array}{l}\text { perceived sleep } \\
\text { deprivation }\end{array}$ & days/week & $2.9(2.0)$ & $2.9(2.0)$ & \\
\hline $\begin{array}{l}\text { satisfied with } \\
\text { pre-pregn weight }\end{array}$ & $\begin{array}{l}\text { 1=very dissatisfied; } \\
\text { 7=very satisfied }\end{array}$ & $5.1(1.4)$ & $5.1(1.4)$ & \\
\hline $\begin{array}{l}\text { estimated pre- } \\
\text { pregnancy BMI }\end{array}$ & $\begin{array}{l}\text { underestimated } \\
\text { correct } \\
\text { overestimated }\end{array}$ & $\begin{array}{l}64(16.2) \\
320(80.8) \\
12(3.0) \\
\end{array}$ & & \\
\hline smoking & $\begin{array}{l}\text { stopped } \\
\text { continued } \\
\text { non-smoking }\end{array}$ & $\begin{array}{l}57(14.4) \\
21(5.3) \\
318(80.3)\end{array}$ & $\begin{array}{l}60(13.2) \\
25(5.5) \\
370(81.3)\end{array}$ & $\begin{array}{l}26.6 \% \text { smokers } \\
\text { in women } 25-45 \\
\text { yrs }^{158}\end{array}$ \\
\hline GWG-goal & no GWG goal & $298(75.3)$ & $338(74.4)^{\mathrm{a}}$ & \\
\hline $\begin{array}{l}\text { GWG advice from } \\
\text { midwife }\end{array}$ & no advice & $343(86.6)$ & 401 (88.1) & \\
\hline
\end{tabular}




\subsubsection{Gestational weight gain (GWG) in relation to Body Mass Index (BMI) category}

Table 3.3 shows the Pre-pregnancy BMI and GWG-IOM categories for the 396 women having no missing values in a crosstab. Furthermore, BMI categories of non-pregnant women between the age of 20 to 30 and between the age of 30 to 40 are provided as reference. With $2.8 \%$ of women who were underweight and $11.0 \%$ who were obese, women in our sample were less often underweight and more often obese in comparison to the general female population. About $42 \%$ of the women gained weight within the IOM-guidelines. Two thirds of the women in our study sample had a normal pre-pregnancy BMI (65.9\%). Almost half of them gained weight within the guidelines. This means that in total less than one third of healthy pregnant women (128 out of 396) had a healthy BMI at the start of pregnancy and a healthy weight gain during pregnancy as well (Table 3.3).

Table 3.3 Gestational weight gain (GWG) according to Institute of Medicine (IOM)-guidelines by pre-pregnancy Body Mass Index (BMI)

\begin{tabular}{|c|c|c|c|c|c|}
\hline $\begin{array}{l}\text { GWG-IOM } \rightarrow \\
\text { pre-pregnancy BMI } \\
\downarrow\end{array}$ & $\begin{array}{c}\text { below IOM } \\
\text { n (\%) }\end{array}$ & $\begin{array}{c}\text { within IOM } \\
\text { n (\%) }\end{array}$ & $\begin{array}{c}\text { above IOM } \\
\text { n (\%) }\end{array}$ & $\begin{array}{l}\text { Total } \\
\text { n (\%) }\end{array}$ & $\begin{array}{l}\text { Dutch } 2013 \\
\text { population women } \\
20-30 \mathrm{yrs} / 30-40 \mathrm{yrs} \\
(\%)^{158}\end{array}$ \\
\hline underweight & $2(18.2)$ & $2(18.2)$ & $7(63.6)$ & $11(2.8)$ & $5.5 / 3.5$ \\
\hline normal weight & $32(12.3)$ & $128(49.0)$ & $101(38.7)$ & 261 (65.9) & $86.2 / 57.8$ \\
\hline overweight & $14(17.5)$ & $24(30.0)$ & $42(52.5)$ & $80(20.2)$ & $5.4 / 27.8$ \\
\hline obese & 7 (15.9) & $14(31.8)$ & $23(52.3)$ & 44 (11.0) & $2.8 / 11.0$ \\
\hline Total & 55 (13.9) & $168(42.4)$ & 173 (43.7) & $396(100)$ & $100 / 100$ \\
\hline
\end{tabular}

Abbreviations: BMI, Body Mass Index; GWG, Gestational Weight Gain; IOM, Institute of Medicine

\subsubsection{Associations with healthy gestational weight gain (GWG)}

Table 3.4 shows the outcome of the multinomial regression analysis. According to pseudo $r$ square of Nagelkerke our regression model explained $18 \%$ of variance of GWG-IOM. Decline in $P A$ was significantly associated with GWG above compared to within the IOM-guidelines. Prepregnancy $B M I$, diet, Motivation healthy $P A$ and pre-pregnancy $P A$ were not associated with weight gain within IOM-guidelines compared with weight gains below and above the IOMguidelines.

Ethnicity and stopped smoking were significant covariates related with weight gain within the IOM-guidelines. Non-Caucasian women were five times as likely, and women who stopped smoking were two times as likely, to have a high GWG. Gestational age was almost significantly associated with too high GWG: with every week further into pregnancy, women were 0 to $5 \%$ more likely to exceed the guidelines.

A significant association was found for healthy GWG compared with too low GWG in women who perceived higher sleep deprivation (an increase in Perceived sleep deprivation of one day 
per week gave a $20 \%$ greater chance of GWG within IOM-guidelines) and almost significant values were found for women who stopped smoking (Table 3.4).

Table 3.4 Multinomial regression model of gestational weight (within guidelines is reference) $\mathrm{n}=396$

\begin{tabular}{|c|c|c|c|c|c|}
\hline & & \multicolumn{2}{|c|}{ below versus within IOM } & \multicolumn{2}{|c|}{ above versus within IOM } \\
\hline & & p & OR $(95 \% \mathrm{Cl})$ & $\mathbf{p}$ & OR $(95 \% \mathrm{Cl})$ \\
\hline \multicolumn{2}{|c|}{ pre-pregnancy BMI $\left(\mathrm{kg} / \mathrm{m}^{2}\right)$} & 0.123 & $1.09(0.98-1.23)$ & 0.767 & $1.01(0.93-1.10)$ \\
\hline \multicolumn{2}{|c|}{$\begin{array}{l}\text { vegetable consumption } \\
\text { (grams/week) }\end{array}$} & 0.414 & $0.97(0.89-1.05)$ & 0.179 & $1.04(0.98-1.10)$ \\
\hline \multicolumn{2}{|c|}{ fish-norm (0=not met; $1=$ met) } & 0.870 & $1.11(0.31-3.97)$ & 0.801 & $1.13(0.44-2.86)$ \\
\hline \multicolumn{2}{|c|}{ fruit-norm ( $0=$ not met; $1=$ met) } & 0.533 & $0.81(0.42-1.57)$ & 0.316 & $0.78(0.49-1.26)$ \\
\hline \multicolumn{2}{|c|}{ motivation healthy $\mathrm{PA}^{\mathrm{a}}$} & 0.874 & $0.98(0.73-1.31)$ & 0.317 & $0.90(0.73-1.11)$ \\
\hline \multicolumn{2}{|c|}{$\begin{array}{l}\text { decline in PA ( } 0=\text { not declined; } \\
1=\text { declined })\end{array}$} & 0.526 & $0.80(0.41-1.59)$ & 0.015 & $0.54(0.33-0.89)$ \\
\hline \multicolumn{2}{|c|}{ pre-pregnancy $\mathrm{PA}^{\mathrm{a}}$} & 0.925 & $1.01(0.76-1.36)$ & 0.352 & $1.11(0.89-1.38)$ \\
\hline \multicolumn{2}{|l|}{ age (years) } & 0.407 & $0.96(0.87-1.06)$ & 0.712 & $0.99(0.93-1.05)$ \\
\hline \multicolumn{2}{|c|}{$\begin{array}{l}\text { gestational age at weighing } \\
\text { moment }\end{array}$} & 0.925 & $1.00(0.76-1.36)$ & 0.064 & $1.03(1.00-1.05)$ \\
\hline \multicolumn{2}{|c|}{ parity (0=nulliparous; $1=$ parous) } & 0.866 & $1.06(0.53-2.12)$ & 0.754 & $0.92(0.56-1.53)$ \\
\hline \multicolumn{2}{|c|}{ ethnicity (0=Dutch; 1=non-Dutch) } & 0.716 & $0.76(0.18-3.30)$ & 0.002 & $0.22(0.08-0.56)$ \\
\hline \multicolumn{2}{|c|}{$\begin{array}{l}\text { family income ( } 0=\text { below; } 1=\text { above } \\
\text { modal) }\end{array}$} & 0.779 & $1.12(0.52-2.39)$ & 0.796 & $0.93(0.52-1.66)$ \\
\hline \multirow[t]{3}{*}{ education } & low & 0.718 & $1.33(0.28-6.32)$ & 0.631 & $0.77(0.26-2.26)$ \\
\hline & high & 0.945 & $1.03(0.51-2.08)$ & 0.546 & $0.85(0.51-1.44)$ \\
\hline & middle & & reference & & reference \\
\hline \multirow[t]{3}{*}{ smoking } & stopped & 0.071 & $4.07(0.89-18.61)$ & 0.034 & $0.49(0.25-0.95)$ \\
\hline & continued & 0.448 & $0.60(0.14-2.56)$ & 0.306 & $0.56(0.18-1.71)$ \\
\hline & non-smoking & & reference & & reference \\
\hline \multicolumn{2}{|c|}{ perceived sleep deprivation $^{b}$} & 0.028 & $1.20(1.02-1.41)$ & 0.335 & $1.06(0.94-1.20)$ \\
\hline \multicolumn{2}{|c|}{ satisfied pre-pregnancy weight $^{\mathrm{a}}$} & 0.635 & $1.08(0.78-1.50)$ & 0.564 & $0.94(0.74-1.18)$ \\
\hline \multirow[t]{3}{*}{ perceived BMI } & too low & 0.764 & $1.17(0.41-3.32)$ & 0.102 & $0.55(0.27-1.13)$ \\
\hline & too high & 0.982 & $0.98(0.09-10.75)$ & 0.342 & $0.49(0.11-2.13)$ \\
\hline & adequate & & reference & & reference \\
\hline \multicolumn{2}{|c|}{ GWG-goal (0=not; 1=having) } & 0.881 & $0.94(0.44-2.03)$ & 0.368 & $0.78(0.45-1.34)$ \\
\hline \multicolumn{2}{|c|}{$\begin{array}{l}\text { GWG advice from midwife ( } 0=\text { not; } \\
1=\text { received) }\end{array}$} & 0.534 & $0.74(0.29-1.90)$ & 0.683 & $0.86(0.42-1.76)$ \\
\hline
\end{tabular}

\subsection{Discussion}

The aim of this study was to explore GWG in healthy women in relation to pre-pregnancy $\mathrm{BMI}$, diet, PA and relevant covariates.

We found that a minority of our sample of healthy women had a healthy GWG according to the IOM-guidelines. Too much GWG was more common than too little GWG. From the hypothesised determinants, only a decline in PA was significantly associated with unhealthy 
GWG. Other hypothesised determinants, including pre-pregnancy BMI, dietary determinants (vegetable consumption, fruit-norm and fish-norm), pre-pregnancy PA and the level of motivation to engage in healthy PA, were not significant. Significant covariates were perceived sleep deprivation (for too low GWG), ethnicity and stopped smoking (for too high GWG).

Women whose PA declined during pregnancy had a twofold risk of gaining weight above the guidelines. Given that the PA for more than half of the women declined, this can be seen as a major contribution to the problem of too high GWG. Interestingly, GWG was not related to motivation to engage in healthy PA, nor was it related to the level of pre-pregnancy PA. Reviews on interventions aimed at increasing PA during pregnancy show a significant but small effect on GWG. ${ }^{72,159,160}$ Our study adds evidence that if we are to reduce unhealthy GWG there is an urgent need to better understand declining PA during pregnancy, including the determinants of this decline.

In our sample of healthy women we found no significant associations between high prepregnancy BMI and too high GWG. While it may be more urgent for obese women to have a healthy GWG, our data showed that their likelihood of gaining too much weight was not higher than for women with a normal pre-pregnancy BMI. A recent publication ${ }^{161}$ points to the importance of paying attention to healthy GWG of overweight women in addition to that of obese women. Women with a normal pre-pregnancy BMI significantly less often exceeded the IOM-guidelines than the overweight and obese women in this study. Differences between their findings and ours can be explained by the population studied. Schlaff et al included all pregnant women while we included only healthy pregnant women and excluded overweight and obese women with obstetric problems from our study. ${ }^{161}$ It could be that obese and overweight women with obstetric problems more often gain weight above the guidelines than overweight and obese women not referred to an obstetrician. Moreover, it is also plausible that overweight and obese women exceed the guidelines more often at higher gestational ages. We included women of all gestational ages in our sample, while Schlaff et al measured women's GWG at the end of pregnancy. ${ }^{161}$ Our data suggest that antenatal caregivers must also concern themselves with GWG for women with a normal pre-pregnancy $\mathrm{BMI}$, although the recommended kilos will vary per pre-pregnancy BMI category. Encouraging all women to achieve healthy GWG could have the additional effect of reducing the stigmatization that overweight and obese women already suffer about their weight. ${ }^{162}$ A recent review concludes that the role of nutrition in GWG is largely unknown. ${ }^{163}$ Our study confirms the complicated relationship between diet and GWG. The vegetable, fruit and fish intake of pregnant women was far below recommendations. Nevertheless, the dietary behaviour of our sample was better than non-pregnant women of comparable ages in the Netherlands, ${ }^{129}$ indicating that pregnant women do improve their diet during pregnancy. However, none of the measured dietary behaviours were significantly associated with healthy GWG. Other researchers in the Netherlands have found that women who reported an increase in food intake were almost three times as likely to gain weight above the guidelines. ${ }^{44}$ We suggest that the change of food intake as compared to pre-pregnancy food 
intake could be related to healthy GWG. It could be the case that women who think it is necessary to adapt their lifestyle (including PA and diet) in order to prevent negative consequences of their pregnancy gain more weight than women who do not adapt their lifestyle.

Non-Caucasian women in our study had a fivefold increased risk of gaining weight above the IOM-guidelines as compared to Caucasian participants. Other authors have found similar differences among ethnic groups in relation to $\mathrm{GWG}^{41,164}$ suggesting the need to adapt the IOM-guidelines for different ethnic groups. Bodnar et al. researched this question and concluded that adaptation for ethnicity may not be necessary, as there was no association between ethnicity and the negative pregnancy outcomes generally associated with high weight gain, including babies who are too small or large for their gestational age, pre-term births and planned caesarean sections. ${ }^{165}$

Differences in GWG between Caucasian and non-Caucasian women are explained by different habits and attitudes among ethnic groups. But it is also important to note that ethnic minorities often have fewer resources and poorer health than the Caucasian population. GWG in non-Caucasian populations needs more attention, which is not only a medical task, but also an issue that must be addressed by political, social and structural policies. ${ }^{166}$ Our sample consisted of relatively few non-Caucasian participants, compared to national figures. The number of women gaining weight above the guidelines would likely have been higher if our sample contained more non-Caucasian women.

With regard to stopping smoking, our study confirms other research ${ }^{131}$ showing that stopping smoking increased the risk of too high GWG and probably decreased the risk of too low GWG. Stopping smoking is often correlated with increased consumption of high caloric food. ${ }^{167}$ Prenatal care providers should know that women who stop smoking require additional attention in order to achieve a healthy GWG. An intervention aimed at healthy GWG should contain an element focused on women who stop smoking.

We found that women with higher perceived sleep deprivation had an increased chance of gaining weight below the IOM-guidelines. On average, women reported sleep deprivation for nearly three nights per week. The experience of sleep deprivation by pregnant women has been associated with too high weight gain in other studies, especially during the first trimester. ${ }^{44,168}$ Less known is the association of increased sleep with too high $\mathrm{GWG}^{53}$ and the association between sleep deprivation and too low GWG. ${ }^{169}$ There is evidence with respect to non-pregnant women to show that an optimum of sleeping hours is important to weight management. ${ }^{170}$ We suggest that an optimum of sleep contributes to healthy GWG in pregnancy as well, and that healthy sleep could prevent both high GWG and low GWG. Our findings show that all types of women are subject to unhealthy GWG. With the exception of women who stopped smoking and non-Caucasian women, there is no reason to focus on special groups when promoting healthy GWG. An intervention aimed at increasing the proportion of women with a healthy GWG needs to be focused on all women, irrespective of their characteristics. 
Our sample included a relatively small number of women with low levels of education, women who smoked and women of non-Caucasian origin, a problem common in this kind of research. ${ }^{44}$ Except for these limitations, our sample is representative of the healthy pregnant Dutch population ${ }^{143}$ and with the necessary caution our results can be generalised to healthy populations in other Western countries as well.

The strength of our study is its focus on the large group of healthy pregnant women as opposed to the subgroups of obese or diabetic pregnant women. From a public health perspective this is an important group where the potential for health benefits is large. What is found to be important for healthy pregnant women forms the foundation of recommendations for all pregnant women. Furthermore, our sample was large enough to test multiple covariates together in the regression model. Although we did not measure actual PA, we included the proxy variable Motivation to engage in healthy PA, based on the Self Determination Theory. ${ }^{155}$ We realize that motivation to perform healthy PA is a substitute and not the same as the behaviour itself, especially for pregnant women who often report complaints during pregnancy that prevent them from being more physically active. In addition, we used the measures of pre-pregnancy PA and decline in PA.

We used self-reported data for pre-pregnancy weight and for weight during pregnancy. This is a limitation because some women will have underreported their weight. Our percentages of too high GWG would have been higher if pre-pregnancy weight had been reported more objectively; some participants would have been categorised in a higher BMI category and advised to achieve lower weight gain.

In the FFQ-vegetables, we substituted photos for spoons. We do not know what effect this has on the validity of the questionnaire. Although we expect that the amount of vegetables is easier to estimate with the use of photos of vegetables than imagining the number of spoons, further validation is recommended.

We were able to explain $16 \%$ of the variance of healthy GWG, suggesting that a number of determinants of GWG are still unknown. Hill offered a conceptual model to explain GWG. ${ }^{43}$ In this model body-perception and psychosocial determinants play a role. Other researchers found a relationship between anxiety and GWG among obese pregnant women. ${ }^{171}$ Social influences and barriers could also be important determinants, as shown in several behavioural models. ${ }^{62}$ A final determinant could be the prenatal classes followed by women in preparation of delivery. Women who were enrolled in the Women, Infants and Children (WIC) program in the United States less often exceeded GWG recommendations ${ }^{161}$ as well as women who received prenatal care in groups (Centering Pregnancy). ${ }^{172}$

\subsection{Conclusion and recommendations}

Healthy GWG (GWG within the IOM-guidelines) occurred in $42 \%$ of all healthy pregnant women and was associated with maintaining a pre-pregnancy PA-level, but not with prepregnancy BMI and diet. The message that women should strive toward a healthy GWG should be given to women from all pre-pregnancy BMI-groups, including women with a 
normal pre-pregnancy BMI. Furthermore, women who need extra attention are non-

Caucasian women and women who stopped smoking. To prevent too high GWG we need to find ways to support women to maintain their PA level during pregnancy. More research is necessary to determine how a decline in PA can be prevented and to increase the explained variance of GWG in the healthy pregnancy population. 


\title{
Chapter 4
}

\section{How and why do healthy pregnant women change their physical activity in pregnancy?}

\begin{abstract}
Astrid Merkx, Marlein Ausems, Luc Budé, Raymond de Vries, Marianne J. Nieuwenhuijze
\end{abstract}

Submitted 


\section{Background}

Reduction of physical activity (PA) during pregnancy is common but undesirable, as it is associated with negative outcomes, including high gestational weight gain. We explored change in five types of activity and the behavioural determinants related to change in PA.

\section{Methods}

Secondary analyses of an exploratory cross sectional survey among healthy pregnant women $(n=455)$ of all gestational ages. We used a hypothetical model, based on the ASE-Model (attitude, social influence, self-efficacy) to construct the questionnaire and analysed our data using descriptive and inferential statistics.

\section{Results}

More than half of the women reported reduction in their PA during pregnancy. Highest reduction was reported in sports and brief rigorous activities, but other types of PA were reduced as well. Reduction of PA was seen more in women who considered themselves as active before pregnancy, women who experienced pregnancy-related barriers, women who were advised to reduce their PA, and multiparous women. Fewer than $5 \%$ increased their PA. Motivation to engage in PA was positively associated with enjoying PA.

\section{Conclusion}

More than half of the pregnant women reduced their PA during pregnancy. Our findings concerning the predictors of PA reduction can be used to develop an evidence-based intervention aimed at encouraging healthy PA during pregnancy. All pregnant women should be informed about the positive effects of staying active and should be encouraged to engage in or to continue moderate intensive activities like walking, biking or swimming. 


\subsection{Introduction}

Physical activity (PA) in pregnancy improves pregnancy outcomes. ${ }^{22,159,173-176}$ Being physically active is associated with lower incidence of pre-eclampsia, gestational diabetes, varicose veins, lower back pain, Caesarean section, post-partum anxiety and depression, adverse birth weight and is associated with improved appetite control, fitness, pain coping during birth and healthy gestational weight gain (GWG). ${ }^{22,159,173-176}$ Conflicting evidence is found in relation to a decrease of nausea, heartburn, round ligament pain, leg cramps, duration of labour, improved sleep experience and quality of life in active women, with some researchers finding a positive and others finding no association. ${ }^{173}$

Canada, the United States of America and the United Kingdom have published national guidelines for PA by pregnant women, based on World Health Organisation (WHO) recommendations. ${ }^{173,177}$ In the Netherlands the WHO guidelines have been used to develop guidelines for PA for the general population, but there are no guidelines for PA by pregnant women. ${ }^{177}$ In this paper, we use the Dutch activity norm -at least five times a week, 30 minutes per day of moderately intense activity, described as "activity where the person needs to breath more and heart rate increases (for instance brisk walking or biking), but the activity is not exhausting" - as the definition of healthy PA for healthy pregnant women. ${ }^{178}$ Globally, around $34 \%$ of women over 15 were insufficiently active in $2008 .{ }^{179}$ When pregnant, many women show a reduction in PA. ${ }^{131,180-182}$ Depending on time, sample and method of measurement, this reduction in PA occurs in as few as $7 \%$ and as many as $69 \%$ of pregnant women. Only a few women increase their PA. ${ }^{182}$

A review of 25 studies of the patterns and determinants of PA in pregnancy, found that a low level of PA is associated with low income, low education, more children in the home, ethnicity other than white and lower pre-pregnancy activity, physical complaints, lack of resources in social environment and lack of social support. ${ }^{180}$ The literature is inconsistent about the influence of parity, employment, pre-pregnancy BMI, age and smoking. Higher self-efficacy expectations and positive beliefs are associated with increased PA in pregnancy. ${ }^{180}$ Level of PA is furthermore associated with the information provided ${ }^{183,184}$ and beliefs about the risks and benefits of PA in pregnancy. ${ }^{185-187}$ Although it is likely that women reduce their PA because of an increased tiredness, a growing belly, and the combination of pregnancy and unwanted types of PA (such as contact sports), reducing PA is undesirable especially for women with low levels of PA before pregnancy. ${ }^{179}$ If these women further reduce their PA, they are at increased risk for negative outcomes of pregnancy and birth. ${ }^{22,159,173-176}$

Most studies based their research on objective measurements of PA, while some explored a subjective perception of change in PA in pregnancy. ${ }^{44}$ While the first method provides a more consistent measure, the latter can be better applicable in prenatal practice. During the day, women have shorter and longer moments of PA, it takes a lot of time to find out the exact types and duration of PA a woman has. It can be easier to ask women whether they perceive a change in their PA in pregnancy, why they changed and how they changed. In a previous study, we discovered that women who perceived a decrease in their PA during pregnancy 
were more likely to have a higher GWG. ${ }^{188}$ This current paper describes the secondary analysis of the data of our previous study, aiming to understand changes in various type of PA in pregnancy and to uncover the factors associated with an overall perceived change in PA in healthy pregnant women. Our goal is to identify what is necessary to build interventions that will prevent the reduction of PA and perhaps stimulate an increase in PA.

\subsection{Methods}

We performed a secondary analyses of data collected in an exploratory cross-sectional survey of a sample of healthy pregnant women (defined as pregnant women not needing specialist obstetric care) of all gestational ages.

\subsubsection{Procedure}

The study was part of the bigger research project "Promoting Healthy Pregnancy" designed to develop an evidence-based intervention to increase the number of women achieving healthy GWG. We worked together with the "Promoting Healthy Pregnancy" Consortium, a group of midwives, other health professionals and researchers. Between September and November 2012, healthy pregnant women of all gestational ages were recruited via 30 midwife-led community practices in the Netherlands. Women who expressed an interest in participating in the study were telephoned by the researcher, who explained the study aim and procedures. Women agreeing to participate were asked to return a written completed consent form. A study questionnaire was then -on request- sent by email. More detailed description of recruitment of participants is described earlier. ${ }^{188}$ The Research Ethics Committee of AtriumOrbis-Zuyd reviewed the study protocol and confirmed that ethical approval was not required.

\subsubsection{Hypothetical model}

We used the Attitude-Social influence-self-Efficacy (ASE)-mode ${ }^{189}$ to explain the change in PA of the participants. The ASE-model is an extension of the frequently used Theory of Planned Behaviour. ${ }^{189-191}$ According to the ASE-model, the particular behaviour is explained by a person's intention to perform the behaviour, which is in turn determined by attitudes (beliefs about the particular behaviour), social influences (perceptions of social norms, social support or pressure and role models) and self-efficacy (a person's expectations regarding her capability to perform that behaviour). ${ }^{189-191}$ Barriers to perform the behaviour can inhibit the behaviour. ${ }^{189-191}$ We defined women's "intention to perform" as "motivation to engage in healthy PA" ${ }^{192}$ We hypothesized that women with higher scores on "motivation to engage in

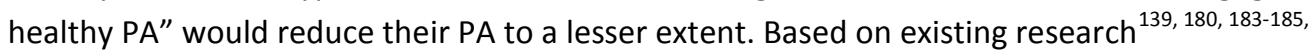
187,193 we hypothesized that (self-reported) pre-pregnancy PA and the degree of eagerness to seek information about pregnancy would influence the change in PA as well. Our hypothetical model is shown in Figure 1. 


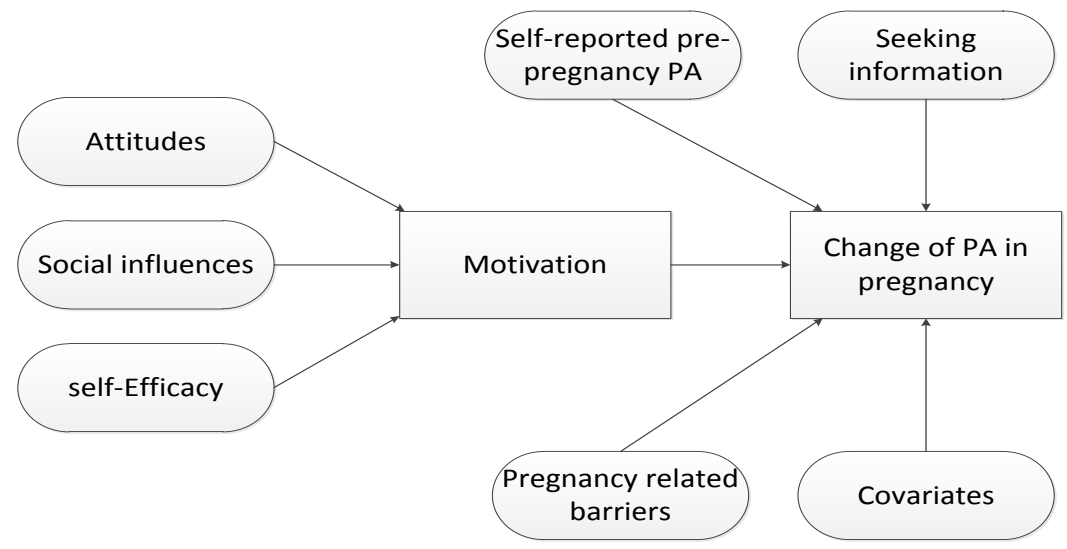

Figure 4.1 Hypothesized model

Our hypothesized model is based on Attitude-Social Influence-self-Efficacy-Model: Motivation to have healthy Physical Activity (PA) (central in figure) is predicted by attitudes, social influences and self-efficacy. Change in PA in pregnancy is predicted by motivation, self-reported pre-pregnancy PA, activity in seeking information about pregnancy, pregnancy related barriers and covariates.

\subsubsection{Questionnaire}

We developed our questionnaire in consultation with midwives, other health professionals, and student midwives to enhance the face validity of our questionnaire. ${ }^{188}$

\section{Demographics/characteristics}

We calculated age using date of birth and date of completing the questionnaire. Using a government definition, ethnicity was Dutch if both parents were born in the Netherlands and otherwise non-Dutch. ${ }^{158}$ Family income was "low" when it was below national modal income (33,000 euro/year) and high if it was above the mode. ${ }^{147}$ Education was "low" for women without a high school diploma, "medium" for women with high school diploma and "high" for women with a university degree. Work was "part-time", "full time" or "no paid job". Parity was "nulliparous" or "parous". Gestational Age (weeks) was calculated using the expected date of birth and the date of completing the questionnaire, Pre-pregnancy Body Mass Index (BMI) was calculated by dividing self-reported pre-pregnancy weight by the self-reported squared height. Having a weight gain goal was "yes" or "no". Smoking was "non-smoking", "stopped" or "continued". We asked participants report their activity in seeking information about pregnancy using a Likert scale (1=very inactive, $7=$ very active). Dummy variables were computed for categorical variables with more than two levels (education, work and smoking), with respectively "medium", "part-time work" and "non-smoking" as reference categories.

\section{Pre-pregnancy PA and Change in PA}

For this secondary analysis we used pre-pregnancy PA and change in PA. Pre-pregnancy PA was measured using the response to a single question measured on a Likert scale (1= very inactive, $7=$ very active). We further asked: did you change your PA in pregnancy? If a participant responded that she had changed her $\mathrm{PA}$, additional questions about the changes 
in her PA were asked. We asked questions about four likely reasons for why they changed their PA (tiredness, different needs, to prepare for another lifestyle, for the health of the baby), measured on a Likert scale (1=totally disagree, 7=totally agree). For the women who changed their PA we also offered questions about five types of activities that may have changed (walking, cycling, sports, brief rigorous activities -such as running for the bus or playing with children- and working in house and garden).

Our primary outcome Change in PA in pregnancy included three categories: "reduced PA", "maintained PA" and "increased PA". Participants were given one point for every type of activity (walking, biking, sports, brief rigorous activities, working in home and garden) they were more active in. They lost one point for every type of activity they were less active in. A total score was computed by summing up the scores of all activities, which could range from 5 to +5 . Women with a negative total score were categorized as "reduced PA"; women with a null-score and women who reported a priori that they did not change their PA were categorized as "maintained PA". Women with a positive score were categorized as "increased PA".

\section{Motivation, ASE-determinants and barriers}

In our questionnaire, we explained "healthy PA" to be moderately intense PA for 30 minutes per day at least 5 times a week before asking related questions. We then asked women to score statements related to: their motivation to engage in healthy PA ("I want to engage in healthy physical activity"), their attitude related to PA in pregnancy (seven items; e.g., "I enjoy to have healthy physical activity in pregnancy"), their social influences related to PA in pregnancy (three items; e.g., "Most people in my environment think it is important that I have healthy physical activity"), and their self-efficacy for PA in pregnancy (three items; e.g., "I manage to have healthy physical activity"). Finally, we asked about pregnancy related barriers to healthy PA (five items; e.g., "I feel too tired to have healthy physical activity"). All of these items were rated on a Likert scale (1=totally disagree, $7=$ totally agree) and are listed in Table 4.4 .

\subsubsection{Analyses}

Our data showed no violation of the assumption of normality. Descriptive statistics were calculated for each of the three subgroups of Change in PA: women with reduced PA, maintained PA, and increased PA. Differences in reasons for changing PA were tested with ANOVA-test. Relationships between pre-pregnancy $P A$ and Change in PA were explored using cross tabulation and Chi-square. Further visual graphs were created for the five subtypes of activities.

Following our model (Figure 1), we performed a multiple linear regression analysis with motivation for healthy $P A$ as dependent variable and the seven attitudes, three social influences, and three self-efficacy items as independent variables. We next performed a multinomial logistic regression analysis with Change in PA as dependent variable (reduced PA versus maintained PA (reference) versus increased PA), and motivation for healthy PA, self- 
reported pre-pregnancy $P A$, and the five pregnancy-related barriers, as independent variables. We included the following covariates: age, ethnicity, family income, education, work, parity, seeking information, gestational age, pre-pregnancy BMI, having a weight gain goal and smoking. Probabilities $<0.05$ were considered significant.

\subsection{Results}

A total of 950 women agreed to receive information about the study, 475 completed the questionnaire (response rate $=50 \%$ ). Twenty cases were excluded because they did not meet the inclusion criteria of being healthy pregnant with a defined gestational age. Of the remaining 455 women, 183 reported they did not change their PA. Of the 272 women who reported they changed their PA in pregnancy, twenty women increased the same number of activities as they reduced, so they were also categorised as "maintained PA". In total, 203 (44.7\%) women maintained their PA, seventeen (3.7\%) women increased their PA and the remaining 235 women (51.6\%) reduced their PA (Figure 4.2).

Descriptives for the total group of respondents and three subgroups of Change in PA (reduced, maintained, increased) are shown in Table 4.1. Mean self-reported pre-pregnancy PA was 4.7 (sd 1.2) on a scale of 1 (very inactive) to 7 (very active).

Table 4.2 shows participants' report of their pre-pregnancy activity in the total group and split up over the primary outcome variable Change in PA. In sum, 76 (16.7\%) women considered themselves pre-pregnancy as inactive, 130 (28.6\%) women as moderately active and 249 $(54.7 \%)$ as active. Active women reduced their PA more often than moderate and inactive women (Chi-square 48.0378; $p<0.00001$ ).

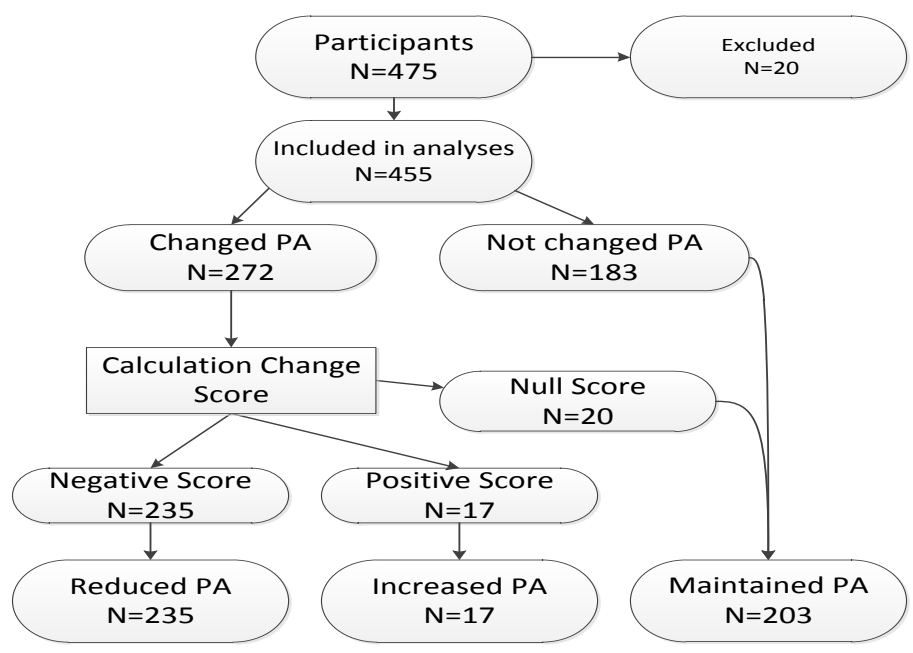

Figure 4.2 Participants according to their change in physical activity (PA)

Twenty women were excluded, because they did not meet the inclusion criteria of being healthy pregnant with a defined gestational age. 
Table 4.1 Demographics and characteristics of participants in mean (sd) or $n(\%)$

\begin{tabular}{|c|c|c|c|c|c|}
\hline & \\
\hline & & $\begin{array}{l}\text { total group } \\
(n=455)\end{array}$ & $\begin{array}{c}\text { reduced PA } \\
(n=235)\end{array}$ & $\begin{array}{l}\text { maintained PA } \\
\quad(n=203)\end{array}$ & $\begin{array}{c}\text { increased PA } \\
(n=17)\end{array}$ \\
\hline \multicolumn{2}{|c|}{$\begin{array}{l}\text { self-reported pre-pregnancy PA, 1= very } \\
\text { inactive, } 7=\text { =very active }\end{array}$} & $4.7(1.2)$ & $5.0(1.1)$ & $4.3(1.3)$ & $4.1(1.3)$ \\
\hline \multicolumn{2}{|l|}{ age, years } & $30.6(3.8)^{a}$ & $30.9(3.7)^{b}$ & $30.2(4.0)$ & $30.2(3.6)$ \\
\hline \multicolumn{2}{|c|}{$\begin{array}{l}\text { ethnicity Dutch (both parents born in } \\
\text { Netherlands) }\end{array}$} & $420(92.3)$ & $220(93.6)$ & $184(90.6)$ & $16(94.1)$ \\
\hline \multicolumn{2}{|c|}{ family income, high (above modal) } & $341(75.3)$ & $180(76.9)$ & $149(73.8)$ & $12(70.6)$ \\
\hline \multirow[t]{3}{*}{ education } & low & $30(6.6)$ & $12(5.1)$ & $15(7.4)$ & $3(17.6)$ \\
\hline & medium & $156(34.3)$ & $71(30.2)$ & $78(38.4)$ & $7(41.2)$ \\
\hline & high & 269 (59.1) & $152(64.7)$ & $110(54.2)$ & $7(41.2)$ \\
\hline \multirow[t]{3}{*}{ work } & fulltime & $201(44.2)$ & $110(46.8)$ & $82(40.4)$ & $9(52.9)$ \\
\hline & part-time & $228(50.1)$ & $113(48.1)$ & $107(52.7)$ & $8(47.1)$ \\
\hline & no paid job & $26(5.7)$ & $12(5.1)$ & $14(6.9)$ & $0(0)$ \\
\hline \multicolumn{2}{|c|}{ parity, nulliparous } & $230(50.5)$ & $126(53.6)$ & $91(44.8)$ & $8(47.1)$ \\
\hline \multicolumn{2}{|c|}{$\begin{array}{l}\text { seeking information, } 1=\text { very inactive, } \\
7=\text { very active }\end{array}$} & $5.1(1.3)$ & $5.0(1.3)$ & $5.1(1.4)$ & $5.6(1.4)$ \\
\hline \multicolumn{2}{|c|}{ gestational age, weeks } & $28.2(8.3)$ & $29.0(8.0)$ & $26.8(8.5)$ & $32.0(7.2)$ \\
\hline \multicolumn{2}{|c|}{ pre-pregnancy BMI, kg/m² } & $24.0(4.2)^{c}$ & $24.0(4.3)^{d}$ & $24.1(4.2)^{\mathrm{e}}$ & $23.7(3.0)$ \\
\hline \multicolumn{2}{|c|}{ having a weight gain goal, yes } & $116(25.6)^{\mathrm{a}}$ & $58(24.8)^{b}$ & $52(25.6)$ & $6(35.3)$ \\
\hline \multirow[t]{3}{*}{ smoking } & stopped smoking & $60(13.2)$ & $26(11.1)$ & $32(15.8)$ & $2(11.8\}$ \\
\hline & continued smoking & $25(5.5)$ & $9(3.8)$ & $14(6.9)$ & $2(11.8)$ \\
\hline & non-smoker & $370(81.3)$ & $200(85.1)$ & $157(77.3)$ & $13(76.5)$ \\
\hline
\end{tabular}

Abbreviations: BMI, Body Mass Index; PA, physical activity

${ }^{a} n=454{ }^{b} n=234{ }^{c} n=426{ }^{d} n=218 \quad{ }^{e} n=191$

Table 4.2 Self-reported pre-pregnancy Physical Activity (PA) versus Change in PA ( $n=455)$

\begin{tabular}{|c|c|c|c|c|c|}
\hline \multirow{4}{*}{ Pre-pregnancy PA } & \multirow{4}{*}{$\%(n)$} & \multirow{2}{*}{\multicolumn{3}{|c|}{ Change in PA }} & \multirow{4}{*}{ Total \% (n) } \\
\hline & & & & & \\
\hline & & reduced & maintained & increased & \\
\hline & & PA & PA & PA & \\
\hline Inactive & & $26.3(20)$ & $69.7(53)$ & $3.9(3)$ & $16.7(76)$ \\
\hline Very inactive & $0.4(2)$ & & & & \\
\hline Inactive & $4.4(18)$ & & & & \\
\hline A bit inactive & $12.3(56)$ & & & & \\
\hline Moderate & & $42.3(55)$ & $51.5(67)$ & $6.2(8)$ & $28.6(130)$ \\
\hline Active & & $64.3(160)$ & $33.3(83)$ & $2.4(6)$ & 54.7 (249) \\
\hline A bit active & $29.9(136)$ & & & & \\
\hline Active & $17.8(81)$ & & & & \\
\hline Very active & $7.0(32)$ & & & & \\
\hline Total \% (n) & & $51.6(235)$ & $44.6(203)$ & 3.7 (17) & $100.0(455)$ \\
\hline
\end{tabular}

Note: Chi-square 48.0378 ( $p<0.00001)$

Abbreviation: PA, physical activity

Reasons for changing PA are shown in Table 4.3. Women who did not change PA did not answer these items. The most noticeable differences between the groups were feeling tired and preparing for another lifestyle. 
Table 4.3 Reasons to change Physical Activity (PA) in pregnancy ( $n=272)$

\begin{tabular}{|c|c|c|c|c|}
\hline \multirow{4}{*}{$\begin{array}{l}\text { I changed my PA, because ... } \\
\text { (1=fully disagree, 7=fully agree) }\end{array}$} & \\
\hline & reduced PA & maintained PA & increased PA & \\
\hline & $n=235$ & $n=20$ & $\mathrm{n}=17$ & ANOVA \\
\hline & Mean (sd) & Mean (sd) & Mean (sd) & p-value \\
\hline ... I feel tired & $4.9(1.7)^{\mathrm{a}}$ & $4.7(1.7)$ & $3.8(1.9)^{b}$ & 0.040 \\
\hline ... I have different needs & $3.6(2.0)^{\mathrm{a}}$ & $4.9(2.0)$ & $3.9(2.2)^{b}$ & 0.024 \\
\hline \multicolumn{5}{|l|}{ concerning PA } \\
\hline ... I think it is good for the baby & $3.4(1.9)^{\mathrm{a}}$ & $4.2(1.6)$ & $3.9(1.9)^{b}$ & 0.123 \\
\hline ... I want to prepare for another & $2.0(1.4)^{\mathrm{C}}$ & $2.6(1.4)$ & $3.9(1.8)^{b}$ & 0.000 \\
\hline lifestyle & & & & \\
\hline
\end{tabular}

Figure 4.3 provides a detailed picture of the number and percentage of women that changed their PA in relation to their pre-pregnancy PA and the change in PA for each type of activity. Because the group who considered themselves as very inactive consisted of only two individuals, these two women were categorised in the inactive group. Increasing PA rarely happened and the greatest reduction was in active sports and brief rigorous activities, including in women who were inactive before pregnancy.

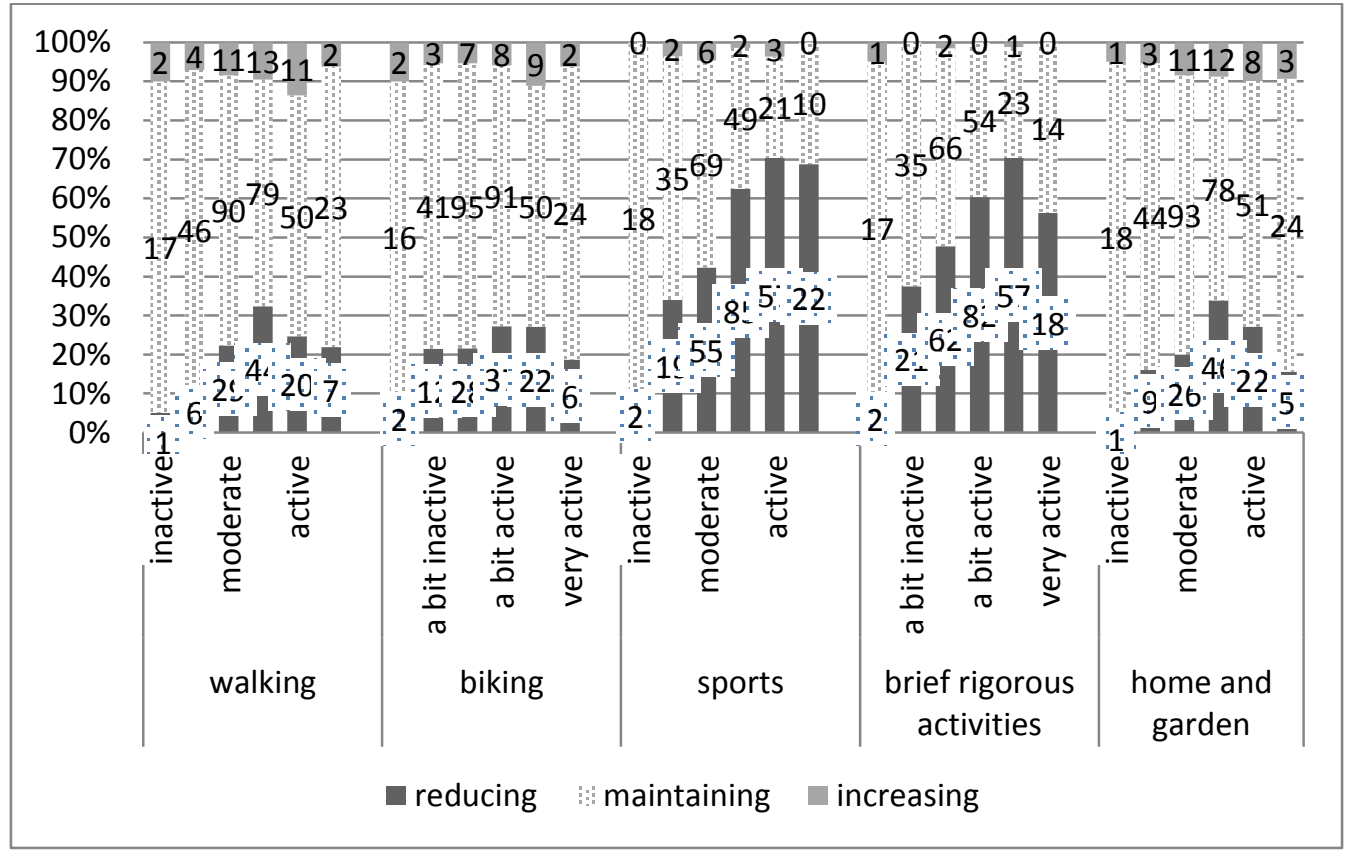

Figure 4.3 Change in Physical Activity (PA)

Percentages of women who reduced, maintained or increased physical activity (PA) in relation to pre-pregnancy PA are visual in bars for the different type of PA $(n=455)$. The numbers provided in the bars refer to the number of women in that category. 
Table 4.4 shows the mean scores for motivation to engage in healthy PA, the ASEdeterminants related to $P A$, and the pregnancy related barriers for the three subgroups. Women who increased their PA had a very high motivation. Women who reduced PA had a higher motivation than women who maintained their PA. Women who increased PA seemed to differ from their counterparts who reduced PA in some attitudes, self-efficacy expectations and pregnancy related barriers.

Table 4.4 Means of attitude, social influence, self-efficacy determinants and barriers related to healthy Physical Activity (PA) in mean of Likertscale (1=completely disagree, $7=$ completely agree) (sd) $(n=455)$

\begin{tabular}{|c|c|c|c|}
\hline PA & $\begin{array}{c}\text { reduced } \\
(n=235)\end{array}$ & $\begin{array}{c}\text { maintained } \\
(n=203)\end{array}$ & $\begin{array}{c}\text { increased } \\
(n=17)\end{array}$ \\
\hline Motivation healthy PA & $5.8(1.1)^{\mathrm{a}}$ & $5.3(1.3)$ & $6.3(0.8)$ \\
\hline \multicolumn{4}{|l|}{ Attitudes } \\
\hline I enjoy to have healthy PA in pregnancy & $5.2(1.6)^{a}$ & $4.5(1.5)$ & $6.1(1.1)$ \\
\hline Healthy PA contributes to be fit for giving birth & $5.8(1.1)^{\mathrm{a}}$ & $5.5(1.3)$ & 5.7 (0.9) \\
\hline Healthy PA in pregnancy is nonsense & $1.6(1.0)^{\mathrm{a}}$ & $1.8(1.0)$ & $1.5(1.2)$ \\
\hline It is difficult to achieve healthy PA in pregnancy & $4.6(1.9)^{\mathrm{a}}$ & $4.0(1.9)$ & 3.5 (1.9) \\
\hline Having no healthy PA makes me feel guilty & $3.4(1.7)^{\mathrm{a}}$ & $3.2(1.7)$ & $3.9(2.3)$ \\
\hline Having too much PA makes me feel guilty & $1.6(1.2)^{\mathrm{a}}$ & $1.6(0.9)$ & $3.3(2.1)$ \\
\hline PA harms the health of the baby & $3.2(1.8)^{\mathrm{a}}$ & $2.8(1.7)$ & $1.2(0.4)$ \\
\hline \multicolumn{4}{|l|}{ Social influence } \\
\hline Most people in my environment think I should have healthy PA & $4.1(1.5)^{\mathrm{a}}$ & $3.8(1.6)$ & $4.5(1.6)$ \\
\hline Most people in my environment have healthy PA & $4.7(1.3)^{\mathrm{a}}$ & $4.3(1.4)$ & $4.5(1.7)$ \\
\hline My midwife supports me to have healthy PA in pregnancy & $3.2(1.5)^{\mathrm{a}}$ & $3.4(1.4)$ & $3.0(1.7)$ \\
\hline \multicolumn{4}{|l|}{ Self-efficacy } \\
\hline I manage to have healthy PA & $3.6(1.7)^{\mathrm{a}}$ & $4.0(1.7)$ & $4.8(1.5)$ \\
\hline I think I have healthy PA & $3.8(1.7)^{\mathrm{a}}$ & $4.1(1.7)$ & $4.8(1.7)$ \\
\hline I think I have better PA than other pregnant women & $3.4(1.6)^{\mathrm{a}}$ & $3.3(1.6)^{b}$ & $3.8(1.8)$ \\
\hline \multicolumn{4}{|l|}{ Pregnancy related barriers } \\
\hline I got the advice to stop favourite sport / PA & $3.5(2.3)^{\mathrm{c}}$ & $2.2(1.8)^{b}$ & $1.8(1.6)$ \\
\hline I experience lack of instruction for healthy PA & $2.0(1.4)^{d}$ & $2.0(1.4)^{b}$ & $1.6(0.9)$ \\
\hline I experience lack of guidance for healthy PA & $2.1(1.4)^{d}$ & $2.0(1.5)^{b}$ & $1.6(0.9)$ \\
\hline Pain while moving prevents me to have PA & $4.5(2.1)^{c}$ & $3.4(2.1)^{b}$ & $4.0(2.1)$ \\
\hline I feel too tired to have healthy PA & $5.2(1.6)^{\mathrm{c}}$ & $4.5(1.9)^{b}$ & $4.6(2.0)$ \\
\hline
\end{tabular}

Abbreviation: PA, physical activity

${ }^{a} n=233{ }^{b} n=202{ }^{c} n=234{ }^{d} n=232$

Table 4.5 shows the outcomes of the linear regression model with Motivation for healthy PA as dependent variable. Four of the seven attitudes were significantly related to motivation, while social influence and self-efficacy were not significantly associated.

Table 4.5 Multiple linear regression model with Motivation for Healthy Physical Activity (PA) $(n=434)$

\begin{tabular}{lcc}
\cline { 2 - 3 } & P value & standardized beta \\
\hline Attitudes & & \\
I enjoy having healthy PA & 0.000 & 0.507
\end{tabular}




\begin{tabular}{lcc} 
Healthy PA makes me fit for birth & $\mathbf{0 . 0 0 8}$ & $\mathbf{0 . 0 8 9}$ \\
Healthy PA is nonsense & $\mathbf{0 . 0 0 0}$ & $\mathbf{- 0 . 2 7 6}$ \\
It is difficult to achieve healthy PA & $\mathbf{0 . 0 0 0}$ & $\mathbf{0 . 1 0 2}$ \\
Not having PA makes me feel guilty & 0.572 & 0.014 \\
Too much PA makes me feel guilty & 0.421 & 0.018 \\
PA harms baby & 0.112 & -0.054 \\
\hline Social influence & & 0.044 \\
Others stimulate me to have healthy PA & 0.102 & 0.035 \\
Others have healthy PA & 0.235 & 0.045 \\
Midwife supports me to have healthy PA & 0.093 & \\
\hline Self-efficacy & & -0.062 \\
I manage to have healthy PA & 0.163 & 0.043 \\
I think I have healthy PA & 0.300 & 0.025 \\
I do better than others & 0.404 &
\end{tabular}

Note: $R^{2}=0.656$

Abbreviation: PA, physical activity

The number of women with increased PA was too small ( $n=17 ; 4.7 \%)$ to perform a multinomial regression analysis. Instead, we performed a logistic analysis of "reduced PA" compared to "maintained PA". Pseudo $\mathrm{R}^{2}$ was .279 and significant determinants were motivation, pre-pregnancy PA, advice to stop PA, pain, tiredness, lack of instruction, age, seeking information, and parity (Table 4.6).

Table 4.6 Logistic regression model of Change in PA (reduced $(=1)$ versus maintained $(=0)$ ) $(\mathrm{n}=400)$, Odds Ratio's (OR) with 95\% Confidential Intervals (Cl)

$P$ value

OR $(95 \% \mathrm{Cl})$

\begin{tabular}{|c|c|c|c|}
\hline \multicolumn{2}{|c|}{ Motivation healthy PA } & 0.014 & $1.31(1.06-1.62)$ \\
\hline \multicolumn{2}{|c|}{ Pre-pregnancy PA } & 0.000 & $1.78(1.41-2.26)$ \\
\hline \multicolumn{2}{|c|}{ Advice to stop favorite sport / PA } & 0.000 & $1.30(1.12-1.50)$ \\
\hline \multicolumn{2}{|c|}{ Pain while moving } & 0.007 & $1.21(1.05-1.39)$ \\
\hline \multicolumn{2}{|l|}{ Tiredness } & 0.000 & $1.41(1.19-1.66)$ \\
\hline \multicolumn{2}{|c|}{ Lack of instruction } & 0.558 & $0.89(0.61-1.31)$ \\
\hline \multicolumn{2}{|c|}{ Lack of guidance } & 0.289 & $0.83(0.58-1.17)$ \\
\hline \multicolumn{2}{|l|}{ Age } & 0.025 & $1.08(1.01-1.05)$ \\
\hline \multicolumn{2}{|c|}{ Seeking information } & 0.000 & $0.69(0.56-0.84)$ \\
\hline \multicolumn{2}{|c|}{ Parity (1=multiparous) } & 0.033 & $1.99(1.06-3.76)$ \\
\hline \multicolumn{2}{|c|}{ Ethnicity (1=non-Dutch) } & 0.319 & $0.64(0.26-1.54)$ \\
\hline \multicolumn{2}{|c|}{ Income (1=high income) } & 0.291 & $1.38(0.76-2.51)$ \\
\hline \multicolumn{4}{|c|}{$\begin{array}{ll}\text { Education } & \text { medium (ref) }\end{array}$} \\
\hline \multicolumn{2}{|c|}{$\begin{array}{ll} & \text { low } \\
\end{array}$} & 0.997 & $1.00(0.33-3.00)$ \\
\hline & & 0.108 & $0.64(0.37-1.10)$ \\
\hline \multicolumn{4}{|c|}{$\begin{array}{ll}\text { Work } & \text { part time (ref) }\end{array}$} \\
\hline & full time & 0.961 & $0.99(0.54-1.81)$ \\
\hline & no paid job & 0.353 & $1.66(0.57-4.83)$ \\
\hline \multicolumn{2}{|c|}{ Gestational age } & 0.164 & $1.02(0.99-1.05)$ \\
\hline \multicolumn{2}{|c|}{ Pre-pregnancy Body Mass Index } & 0.596 & $1.02(0.96-1.08)$ \\
\hline \multicolumn{2}{|c|}{ Having a weight gain goal (1=yes) } & 0.993 & $1.00(0.57-1.74)$ \\
\hline Smoking & no smoking (ref) & & \\
\hline
\end{tabular}


continued smoking

0.167

$2.25(0.71-7.08)$

Note: $\mathrm{R}^{2}=.279$ (Cox and Snell), .372 (Nagelkerke) $\chi^{2}(\mathrm{df21})=130.597 \mathrm{p}=0.000$

Abbreviations: $\mathrm{Cl}$, confidence interval; OR, Odds ratio; PA, physical activity

\subsection{Discussion}

We wished to learn how healthy pregnant women change their PA and the reasons associated with a change in PA. More than half of women reduced their PA-level during pregnancy. Only a small minority of our sample increased their PA. In our model (Figure 1), we hypothesized several determinants of the change in PA. Our results partially confirmed our theoretical model for reduced PA.

Positive attitudes (e.g., enjoying PA) were, as expected, positively associated with motivation and negative attitudes (e.g. PA is nonsense) were negatively associated with motivation. The most important attitude was "enjoying healthy PA", meaning that PA can likely be promoted by stressing the joy of it. Social influences and self-efficacy were not associated with motivation. Among Latino women, other researchers found a positive association between social support and levels of PA in pregnancy and post-partum. ${ }^{194}$

Interestingly, having a higher motivation to engage in healthy PA was significantly associated with a higher likelihood of reducing PA. We cannot explain this relationship, but we speculate that this may be the result of setting unrealistic goals, or the influence of barriers overruled the influence of the motivation. Although we did not include women with increased PA in our regression analyses, we did notice that women with increased PA also reported higher scores on motivation to engage in healthy PA, and other positive attitudes related to staying active. Further research is needed to understand the factors that interact with motivation to influence level of PA.

High levels of pre-pregnancy PA were associated with reduced PA. Our assumption that women would replace their sports in pregnancy by another type of PA was not confirmed by our data. Women often reduced their PA and rarely increased another type of PA such as walking or biking. This is a troublesome finding, since a significant amount of women of childbearing age engage in the recommended amount of PA. ${ }^{179}$

As hypothesized, and as found in other studies, pregnancy related barriers such as tiredness, pain, and growing belly are associated with a reduction and/or low levels of PA. ${ }^{195-197}$ Women who reported tiredness or pain were significantly more likely to reduce their PA. Women with higher scores on "it is difficult to achieve healthy PA" were more often found in the group who reduced their PA. Many sports and rigorous activities are difficult to maintain when pregnant because of changing balance, growing belly and perceived risks of hurting the baby. ${ }^{198}$ Consistent with findings from others, we also found that multiparous women and older women were more likely to reduce their PA. ${ }^{199-201}$

A serious point of concern in our results is that we found that women who were advised to stop their favourite sport/PA were more likely to reduce their PA. Indeed, it is necessary to stop dangerous sports during pregnancy but it is not necessary to reduce PA in general. 
Pregnant women report they are advised to slow down and are not told by their care provider that PA is important. ${ }^{183}$ Antenatal care providers, including midwives, confirm they give low priority to motivating pregnant women to have healthy levels of PA and they often give women advice to slow down. ${ }^{185,202-205}$ Since prenatal PA is associated with better outcomes, ${ }^{22,}$ 159, 173-176 future interventions should aim to increase knowledge in both midwives and pregnant women and should emphasize the importance of healthy PA (e.g., moderate active activities including walking, biking, swimming) during pregnancy. Midwives may also advise women to slow down because of their concern about the growing psychological complaints women experience. ${ }^{205,206}$ Midwives likely mean slowing down as "de-stressing", but it gets interpreted as "sitting still". Where the first has the intention to empower women to make their own choices, and to listen to their pregnant body, the latter has the intention to sit down and relax physically. Maintaining or increasing PA in pregnancy can in fact help to reduce stress. ${ }^{171,207,208}$ In this respect slowing down should mean: avoid stress and enjoy PA. ${ }^{171}$ Further research should be done to verify the extent and consequences of this confusion.

Women with higher scores on seeking information regarding pregnancy had a lower likelihood of reducing PA. Consumer information available on various internet sites tells women to maintain PA. Furthermore, practical ways to maintain or increase PA in pregnancy are given as well as links to professionals providing specific courses for pregnant women in which PA is involved. ${ }^{178}$ In earlier studies, women expressed an unfulfilled need for pro-active information and support from their care provider. ${ }^{209}$ Our study shows that pregnant women who seek this information on their own have less reduction in PA.

PA is modifiable ${ }^{207}$ and pregnancy is seen as window of opportunity to improve women's health. ${ }^{210}$ We recommend that health professionals pro-actively ask women what their PA level was before pregnancy and encourage women to be physically active in pregnancy. When the kinds of PA women engage in before pregnancy is not appropriate (e.g., contact sports), women should be encouraged to seek an alternative type of PA. Changing PA requires change of daily life routine, which is not always easy to accomplish. ${ }^{211}$ Since the midwife is an important source of information and support, she can discuss this issue with pregnant women and suggest options for staying or becoming physical active. ${ }^{209}$ Despite pain and tiredness, women should be encouraged to sustain or improve their level of PA. Walking and biking can be done very easily during pregnancy. These low cost and easy to follow activities can be introduced as medical prescription for women who have physical complaints of tiredness and pain. Indeed, women tend to follow midwives' advice as this study confirms. Swimming and special meetings for pregnant women (e.g., Mom in Balance, Centering Pregnancy) are also good options but may incur unaffordable costs. To prevent women reducing their PA, care providers should emphasize the fun of PA and stress its importance, while at the same time providing women with information on how to cope with pregnancy related barriers. Down et al. call for a paradigm shift in professionals, encouraging them to focus "more broadly on promoting PA before, during and after pregnancy". ${ }^{174}$ Since inequity is an important barrier to 
public health, ${ }^{166}$ municipalities and health insurance companies should consider covering the cost of PA sessions for pregnant women.

\subsubsection{Strengths and limitations}

The strength of our study is that we focussed on healthy pregnant women instead of subgroups like obese or diabetic pregnant women. Healthy pregnant women make up the vast majority of expectant mothers and thus from a public health perspective, this is an important group for preventing illness in the long term.

We are not sure that we captured all types of PA in our outcome measures and we did not have access to a validated questionnaire. Instead, we used the ASE-model, which provided a solid theoretical starting point for our study. Furthermore we validated our items by using the expertise of our Consortium group of midwives, other health care providers, and researchers. We used self-reported PA. A self-reported change in PA does not imply that a woman did not meet the activity norm during pregnancy. However, as one third of adult women do not meet the activity norm, ${ }^{179}$ we assume that PA reduction during pregnancy also implies insufficient PA for a considerable portion of these women. Objective measures of PA would be more accurate, but given the limitations of time and funding we had no valid way to objectively measure PA in pregnant women. ${ }^{151,152}$ Longitudinal research before and during pregnancy with more objective measurements is necessary to understand PA during pregnancy more extensively.

Our sample included a relatively small number of women with low levels of education, women who smoked, and non-Dutch women, a problem that frequently occurs in this kind of research. ${ }^{44}$ With those exceptions, our sample is representative of the healthy pregnant Dutch population ${ }^{143}$ allowing our findings to be generalised - albeit cautiously - to healthy pregnant women in other Western countries.

\subsubsection{Conclusion and clinical implications}

More than half of the women in our study reported reducing their PA in pregnancy, mostly in sports and brief rigorous activities. Reduction of PA was seen more in women who considered themselves as active before pregnancy, women who experienced pregnancy-related barriers, women who were advised to reduce their PA and multiparous women. Instead of advising women to slow down when women feel stressed or tired, we recommend that antenatal care providers invest in efforts to stimulate healthy PA, and prevent a reduction of PA in pregnant women. Because women experiencing pregnancy related barriers have more difficulties to have healthy PA, care providers must be competent to address these barriers, and to help women coping with the barriers. An available option is to refer to locally accessible and nice PA programs, with low thresholds (e.g., pregnancy courses that contain a considerable amount of PA for low costs). 


\section{Chapter 5}

\section{Dutch midwives' behaviour and determinants in promoting healthy gestational weight gain, phase 1: a qualitative approach}

Astrid Merkx, Marlein Ausems, Luc Budé, Raymond de Vries, Marianne J. Nieuwenhuijze

International Journal of Childbirth 2015; 5(3):126-38 


\section{Background}

A significant contributor to the global threat of obesity is excessive Gestational Weight Gain (GWG). The aim of this article is to explore Dutch primary care midwives' behaviours in promoting healthy GWG.

\section{Methods}

We used the Attitude-Social Influence-Self-Efficacy (ASE)-model to guide interviews with a purposive sample of six midwives working in primary care.

\section{Results}

Midwives reported activities in three areas related to GWG: GWG-Monitoring (weighing and discussing GWG), Diet-Education and to a lesser degree Physical Activity-Education. The determinants from the ASE-model were confirmed and other relevant determinants, including midwives' perception of their role in health promotion, were added.

\section{Practice Implications}

The identified determinants can be used for quantitative research. Quantitative research is necessary to identify the magnitude of the determinants associated with midwives' behaviour in promoting healthy GWG. 


\subsection{Introduction}

High Gestational Weight Gain (GWG) is associated with later overweight and obesity amongst women and their offspring. ${ }^{2,3,5}$ Obesity is seen as a global threat to public health as it is related to major health problems, including diabetes and coronary heart disease. ${ }^{7}$ Several studies have found that both high and low GWG are positively associated with problems during pregnancy and birth. 6, 19,122 The Institute of Medicine (IOM)-guidelines recommend minimum and maximum GWG for women based on pre-pregnancy Body Mass Index (BMI). ${ }^{6}$ The percentage of women in high-income countries who gain weight within these recommendations varies from 21.6 to $48.7 \% .{ }^{41,45,126}$ The incidence of pregnant woman who gain weight below (19\%), within (44\%) and above the guidelines $(38 \%)$ in the Netherlands also demonstrates a clear need to focus on healthy GWG in order to improve the health prospects of mothers and their offspring. ${ }^{44}$ It is as yet unclear how midwives anticipate and adapt to the growing incidence of unhealthy GWG. ${ }^{212-214} \mathrm{~A}$ review of the literature on GWG reveals that we do not yet know what the best intervention is for promoting healthy GWG. ${ }^{6,22,145}$ Three professional behaviours are frequently mentioned as relevant, however: GWG-Monitoring, Diet-Education and Physical Activity (PA)-Education. 6, 60, 215, 216 Prenatal care providers are the preferred professionals to perform these monitoring and educational behaviours. However, to date there is no research on interventions aimed at prenatal care providers and on how best to support them in performing these monitoring and educational behaviours. ${ }^{217}$ When designing an evidence-based intervention to effectively influence the monitoring and educational behaviours of prenatal care providers, it would seem crucial that the intervention be tailored to their current practices. ${ }^{62}$ It is therefore necessary to gain insight into the factors that determine prenatal care providers' behaviour in promoting healthy GWG.

Maternity care in the Netherlands is organized at three levels. ${ }^{218}$ The first level (primary care) is provided in the community and is available to all women with a healthy pregnancy, birth and postpartum term. The second level (secondary care) is provided in all hospitals and is available to women and babies who encounter problems during pregnancy, birth or during the postpartum term. The third level (tertiary care) is provided in specialized hospitals which have expertise and facilities for specialized obstetric and neonatal care. Midwives can work at all three levels. When they work in secondary or tertiary care, they are employed by the hospital and work in close cooperation and share responsibilities with obstetricians, nurses and neonatologists. In total, $83 \%$ of Dutch working midwives work in primary care. ${ }^{219}$ Most of these primary care midwives work in an autonomous practice in cooperation with other midwives (82.3\%), some midwives have an autonomous solo practice (5.2\%) and others work as an employee in a practice of one or more autonomous midwives (12.5\%). ${ }^{219}$

To earn a full-time income, midwives are required by the compensation rules for insured care to carry a workload of 105 full cases per year. ${ }^{220}$ Forty percent of all midwives with an autonomous practice carry fewer than 105 cases per year and are considered to work parttime, but many work full-time nonetheless. ${ }^{219}$ Unlike midwives in some countries who assist physicians in delivering maternal care, Dutch midwives provide full maternity care (including 
education and risk selection), consisting of prenatal care at the practice (10-12 meetings), natal care (at home or if the woman prefers in hospital) and postpartum care at the woman's home ( 3 to 6 visits). ${ }^{221,222}$ Administration and practice management take up about one third of the total working time. ${ }^{223}$ Some midwifery practices employ a practice assistant who takes care of e.g. scheduling appointments, measuring weight, blood pressure, administration, providing information. ${ }^{219}$ Approximately $85 \%$ of all pregnant women in the Netherlands start their pregnancy in midwifery practices. ${ }^{143}$ From this high percentage we conclude that midwives play a central role in women's pregnancy and could play an important role in weight management.

Almost all Dutch primary care midwives (98.1\%) are members of the Royal Dutch Organization of Midwives (KNOV) ${ }^{219}$ and follow KNOV-guidelines. ${ }^{218}$ Attention to weight management was recently included in the KNOV's prenatal care guideline $(\mathrm{p} 172) ;{ }^{222}$ midwives are advised to weigh a woman during her first visit, to advise her about a healthy diet, to inform her about normal weight gain (10-15 kilograms), to explain their weighing practice and to invite questions and concerns about weight gain. The KNOV has yet to adopt the IOM-guidelines for GWG and the Dutch guidelines do not include any reference to PA-Education. Most Dutch midwives were not trained to measure bodyweight during pregnancy in their educational years, as bodyweight was not considered a predictor for hypertension disorders. The most recent edition of a key Dutch study book follows neither the KNOV-guidelines nor the IOMguidelines in this respect (pp 295-6). ${ }^{8}$ The Dutch Organization of Obstetrics and Gynaecology (NVOG) developed a guideline about management of obese pregnant women. ${ }^{224}$ In this guideline obstetricians are advised to calculate BMI before or at the start of pregnancy, to provide secondary care for women with a pre-pregnancy BMI higher than $40 \mathrm{~kg} / \mathrm{m}^{2}$, to use a large cuff for measuring blood pressure in obese women, to refer women with a prepregnancy BMI higher than $40 \mathrm{~kg} / \mathrm{m}^{2}$ to an anaesthesiologist for a pre-partum visit and to develop a local guideline on obese women for all care providers involved in maternity care. ${ }^{224}$ The NVOG did not adopt the IOM-guidelines for GWG. In conclusion, the guidelines concerning GWG in the Netherlands are contradictory, as well as complementary and overlapping. It is unclear if prenatal primary care providers in the Netherlands use the midwifery guidelines, the obstetric guidelines, the IOM-guidelines or a combination of these guidelines. It is also unclear what exactly Dutch midwives do to promote healthy GWG, as well as what the determinants are that are associated with their behaviours in promoting healthy GWG.

We focused in our study on how healthy GWG is promoted by primary care midwives and on the determinants related to this behaviour. We focused on midwives working in primary care because they deliver most of the prenatal care in the Netherlands. The information derived from this study can be used to develop an evidence-based intervention for midwives to adapt their behaviours in promoting healthy GWG.

\subsection{Methods}


We performed a qualitative face-to-face study using semi-structured interview questionnaires.

\subsubsection{Participants}

This study is part of the project Promoting Healthy Pregnancy, which aims to provide health benefits for healthy pregnant women. The project is advised by a multi-disciplinary consortium. We used the network of our consortium members to select a purposive sample of six Dutch midwives working in primary care. The aim was to gather information from different types of midwives working in different types of practices. All invited midwives agreed to participate in our study. They were informed about the aim and procedures of the study and they were told that they were free to withdraw at any moment without restrictions. The practice setting of the included midwives varied: one worked alone, two worked with another midwife, two worked in a group of midwives and one midwife worked in a group of midwives in close cooperation with other health professionals. One midwife was pregnant herself; all midwives were mothers. Their working experience ranged from five to twenty-seven years.

\subsubsection{Procedure}

The interviews were scheduled in September 2011 at a time and place convenient for the participants and lasted between 45 and 90 minutes. All interviews were conducted by the same researcher (first author), who was trained in qualitative interviewing. Participants were informed that there were no wrong answers and they were encouraged to reveal anything they wanted to say about the subjects addressed in the interview. The researcher made notes during and after the interviews. At the end of an interview, the interview was summarized for a member check. All interviews were audiotaped and transcribed verbatim.

The study was entered into the Dutch Trial Register under number TC 3543. The Research Ethics Committee of Atrium-Orbis Zuyd confirmed that due to the non-invasive character of the study ethical approval was not required.

\subsubsection{Measurement}

We used a semi-structured questionnaire based on a behavioural model known as the Attitude-Social Influence-Self-Efficacy (ASE)-model (Figure 5.1). ${ }^{189}$ The ASE-model is an extension of the Theory of Planned Behaviour (TPB) ${ }^{189,190}$ 


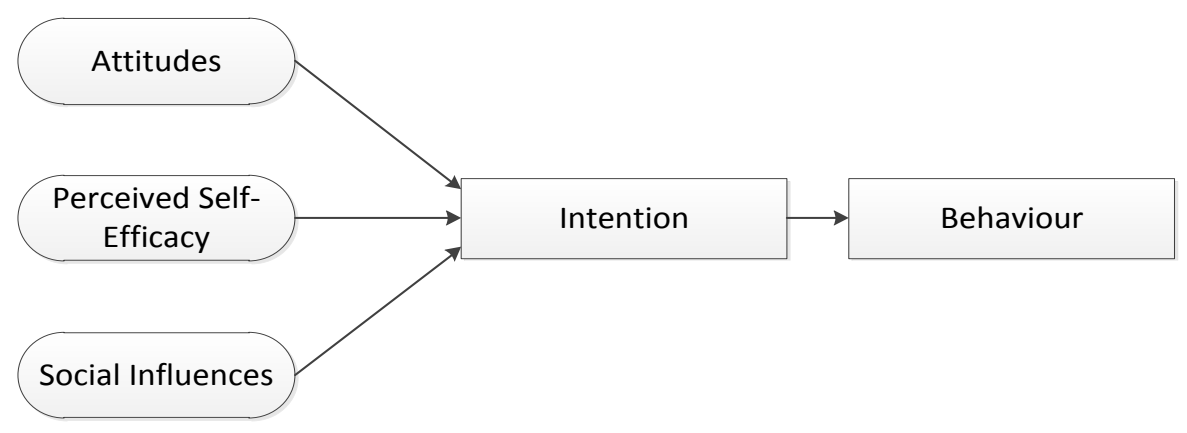

Figure 5.1 Attitude-Social influence-self-Efficacy model

According to the ASE-model, behaviour can be explained by behavioural intention, which in turn is determined by Attitudes (salient beliefs about the particular behaviour), Social influences (perceived norms of important others, perceived social support or pressure and perceived role models) and perceived self-Efficacy (a person's expectations regarding his/her capability to perform the particular behaviour). This model is widely used to explain professional behaviour, including midwives' behaviour. ${ }^{62,225}$

We first asked which midwife behaviours were related to the GWG of their clients. Next, we pro-actively explored the calculation of BMI, weighing women, discussions about GWG (content and methods), diet-education and PA-education. The reasons for midwives' behaviours were also explored. Additionally, the researcher asked pro-actively about the intention to perform the behaviour, about attitudes (e.g., "what is your belief about the importance of promoting a healthy GWG"), social influence (e.g., "what do you believe the client expects from you?") and perceived self-efficacy (e.g., "to what extent do you feel capable of discussing a healthy diet?").

\subsubsection{Analysis}

We used content analysis of incorporated transcripts and interviewers notes (data triangulation). ${ }^{226}$ The transcripts were read, reread and marked independently by the first two authors to achieve a sense of the whole and to identify possible content areas. Quotes were then labelled using constructs of the ASE-model and ASE-determinants were filled with content. During the analysis process a number of determinants not covered by the ASE-model came up. We interpreted the text, discussed it and framed new additional determinants to get a comprehensive understanding of the meaning of the text.

\subsection{Results}

In all interviews a conversation took place in which the midwife openheartedly talked about her underlying opinions, beliefs and barriers. All midwives indicated that they enjoyed the conversation, and during or at the end of the interview they expressed an interest in learning more about GWG and appropriate care for women to achieve a healthy GWG. 
Five of the six midwives were recently involved in the development of local guidelines, as a response to the obstetric guideline about pregnancy management of obese pregnant women mentioned in the introduction of this paper224 The midwives expressed ambivalence about these local obstetric guidelines. They recognized the problems of obesity, such as difficulties in measuring babies' growth and a higher incidence of complications. But at the same time, they did not fully agree with the added value of using secondary care as compared to primary care. They felt that midwives in primary care are also qualified to measure glucose levels, prescribe diets, use large cuffs and make referrals for growth measurements. Furthermore, some midwives mentioned that they only reluctantly agreed with referring women with a BMI higher than $30 \mathrm{~kg} / \mathrm{m}^{2}$ (instead of $40 \mathrm{~kg} / \mathrm{m}^{2}$ ). One midwife recalculated BMI during the second or third trimester and referred women to an obstetrician if the BMI at that time point exceeded $40 \mathrm{~kg} / \mathrm{m}^{2}$. Following local guidelines means that more women were actually referred then required by the NVOG-guidelines. Midwives expressed concern about growing medicalization with the new NVOG-guidelines, but were unable to clearly formulate their objections. Although the aim of the interviews was discussed with the participants prior to the interviews, it was hard to focus on GWG with respect to all pregnant women at the beginning of the interviews because the midwives still had reservation about accepting the guidelines for obese women and they wished to talk about this.

One midwife was involved in a local project that she had initiated which aimed to enhance prenatal care from a public health perspective. The project was at a stage in which other parties (such as dieticians, physiotherapists, psychologists, health insurers and municipalities) were becoming interested in collaboration. Diet- and PA-Education were also part of this project, which was intended to address problems of obesity, maternal distress and teen pregnancy. This midwife was more active in promoting healthy GWG and had a more positive attitude towards promoting healthy GWG than the other participants in our study.

The midwives provided information related to "the constructs of the ASE-model" as well as to "additional determinants". The determinants are presented below, including quotations (italicized in boxes) to illustrate them. In some quotes, additional contextual information is provided between square brackets.

\subsubsection{The constructs of the ASE-model}

The following ASE-themes turned up in the interviews; behaviours (weight-monitoring, dieteducation and PA-education), intention, attitudes, social influences and self-efficacy.

\section{Behaviours}

\section{Monitoring GWG}

All midwives reported that BMI calculation was integrated in the computer-based monitoring system they used for their pregnancy files. To calculate BMI, midwives weighed women during their first visit or asked women what their pre-pregnancy weight was. All midwives 
asked for height and measured only when the woman did not know her height, except for one midwife who always measured during the first visit.

Data on weight were always collected during the first visit. Some of the midwives never measured weight again during the course of pregnancy; others weighed all women during every visit; others weighed every time for specific groups (such as obese women, women with large weight loss in history or women with anorexia in history), or upon request. All midwives were open to women's questions about weight. None of the midwives pro-actively discussed setting a weight gain goal or asked what the woman herself had in mind about weight gain. Midwives advised differently about appropriate weight gain; one midwife mentioned the IOM-guidelines, but did not know the right cut-off points, four mentioned ten to fifteen kilograms for all pregnant women (according to the Dutch midwifery guideline) and one mentioned "not above twenty kilograms". None of the midwives mentioned using a systematic approach to behaviour change, such as motivational interviewing. However, the midwives did describe efforts to help women with their weight gain struggles.

We discuss weight because I always ask about it. Women then say, "well, I just lost weight" or "weight is an issue for me." Then we discuss this. And I follow the women and offer to weigh them if they want. But I tell them that for me it's not necessary. As long as your belly is growing and you and your baby are ok .... and when I don't see the woman about to explode, I don't weigh her. But I think 40 percent want to weigh themselves a couple of times during pregnancy. Just out of curiosity. And with a BMI higher than 30 or 35, women easily agree to being weighed. We motivate them, we tell them we want to keep an eye on their weight gain, just in case ...

\section{Diet-Education}

One midwife with a personal interest in nutrition was very active in Diet-Education. She advised women to eat ten different vegetables per day, to engage in physical activity before a meal, to avoid factory-made foods and to avoid milk products. She shared food recipes with pregnant women and emphasized the importance of eating healthy food. One midwife referred all women with a BMI higher than $27 \mathrm{~kg} / \mathrm{m}^{2}$ to a dietician. The other midwives discussed diet in general, mostly to inform pregnant women about safe eating during pregnancy (i.e. diets that avoid harm to the foetus) and they generally advised "to eat enough fruits and vegetables". If questions arose, or weight gain seemed to be getting out of hand, further recommendations were made to reduce sugar and fat intake and sometimes a visit to a dietician was suggested.

But, when women ask for advice, I say ..." if you want to take care of your weight ... just quit the most obvious things and use common sense. You have common sense! Don't drink soft drinks and ... well ... you can have chocolate, but eat just one piece and not a whole bar. Not 
the whole package of cookies, but just one." ... You know ... that sort of thing ... that everyone knows.

[about women who lost 30 kilograms before pregnancy] I don't want them to focus on their weight, but on healthy diet instead. They're happy when I say that. They know they can do that.

Interviewer: And when they gain too much weight?

No ... well, then I ask, "did you change your diet? Do you eat more, what do you eat more of? Do you eat more sweets or do you sit down with a bag of potato-chips?" That sort of thing, then ... then l ask ... yes ... but in other cases ... no.

\section{PA-Education}

Midwives paid little attention to PA-Education. In most cases they advised women to slow down if they had physical complaints while moving. One midwife changed her mind while discussing this topic.

[About discussing PA] Well, I think it is a good suggestion ... eh ... no ... I don't mention it ,... "do you have your 30 minutes of PA per day?" But I think it's a good suggestion, I can do that!

One midwife encouraged women to be more active. She tailored her advice to the women's daily activities.

You cannot change women's lifestyle completely. But you can say: "well... you can go by bike to do groceries. Or walk with the other children, just go for a walk and do it regularly." Well ... they are open to it.

\section{Intention}

Midwives were very brief about their intentions to promote healthy GWG. They expressed that their intentions and behaviours correspond to each other. They felt that they are autonomous in this respect and that they do what they intend to do.

\section{Attitudes}

In general midwives were moderately positive about Monitoring GWG and Diet-Education. The main reasons to be positive were the prevention of postpartum weight retention and the belief that healthy food contributes to mothers' and babies' health in general. One midwife mentioned that talking about healthy diet was so obvious; she could not explain why she did it. Later in the interview, however, she recognized her perceived role with respect to the health of mother and baby. 
Because ... yes, why do you want to talk about healthy diet? Yes, in general you live on what you eat. I mean ... you cannot live without food. And unhealthy food has damaging effects on health, yes, why do you want to ... It is such a basic question ... I cannot answer that.

Well ... you are there at the beginning of life. And you want to give it a good start. ... It's about giving the pregnant woman good chances for health and a healthy start for her baby.

... We've been midwives for 25 years. We see women from consecutive generations. And we see them doing the same things their parents did. We don't want them to copy the same bad habits when we know they could do better. We seriously want to stop this vicious circle.

One midwife was somewhat reluctant about Diet-Education. She mentioned that pregnancy was the only time that it was normal for a woman to gain weight. She did not want to focus on weight gain for women with a normal, healthy weight.

Then I say ... it's normal to gain weight. It's not necessary to gain twenty kilograms and you don't need to eat for two. You do need to eat healthy. And when you have a moment of craving and you want something sweet ... well ... give in to it once in a while. Because this may be the only time you don't need to watch out that much. For nine months.

Some midwives were less positive about the effectiveness of promoting healthy GWG in women with unhealthy lifestyles, but still wanted to contribute something to the health of the woman and child.

Some women say: "We don't eat healthy because we're always busy and we don't like cooking." Then I advise them to skip the really bad things and take vitamin pills. Well ... they can do that. It is an easy way to handle this. You can't change the entire lifestyle, but you can add something.

In general midwives expressed a positive attitude toward promoting healthy PA. At the same time, they spent no time on it because they seemed to be unaware of the relationship between PA and GWG and the need to discuss PA in this respect. During the interview they became more aware of the benefits of PA.

It is good to pay attention to PA, because in the end, if the woman remains physically active, she'll have a better birth as well. And yes ... probably also a better weight gain.

\section{Social Influences}

Although their behaviours differed, all midwives considered that they were aware of women's needs in Monitoring GWG, Diet-Education and PA-Education. They felt that they were open to questions and provided sufficient information. Most midwives had very little idea about what other midwives offer to women with regard to education on diet and PA. 
Interviewer: Some midwives ask women about their diet and how much PA they engage in. What do you think of that?

Oh, really?? Are there midwives who do that?

One midwife emphasized that her colleagues adhere to the guidelines without having any intrinsic motivation to monitor weight gain.

Other professionals want to work with us. My colleague and I wish that our other colleague midwives would work together like we do with the dietician, the psychologist, the physiotherapist, the municipality, no matter what! They all see the importance. But the people most difficult to convince are our own colleagues. And the obstetricians. They don't think about prevention. Oh no, no. You may think I am being too black and white about this, but that's the way I see it. I just don't see why we have to be told to do this. And our professional board still doesn't recommend weighing women. ... There will come a time that national guidelines and rules require us to pay attention to weight gain ... but it is ... just because there are third parties who say: you have to deliver good quality care ... And then ... [my colleagues will start weighing women] ... I think they [third parties] are right. I can't help it ... yes, it's too bad we have to be told to do this.

\section{Self-Efficacy}

In general, the responding midwives felt confident about their ability to talk about weight gain issues and to discuss diet and PA with women to a certain extent. For detailed dietary advice, however, they preferred to refer to a dietician.

Well, I don't have time for it, but I also want to do it in a professional way. And when it goes in depth ... I'm not the right person. You need to consult a dietician who has studied for this.

One midwife revealed that talking about weight was a touchy topic in the past but that she had learned to discuss it.

No, it is absolutely not difficult to tell women to engage in more PA. No ... we're going to change that in our practice policies.

[About talking with obese women about weight] Yes, I think it's easy now, but in the beginning, [just after implementation of local obstetric guidelines] there were a couple of times that I didn't talk to the woman about her weight during the first visit. I postponed it to the second visit. We all had difficulties with it. Oh, man, we need to say it ... "you have to go to secondary care because you are too fat". But we found a way to do it and I can deal with it now. Well, you are heavy if your $B M I$ is over 40, but ... yes ... at that time, it was really hard for me. 


\subsubsection{Additional determinants}

In addition to the ASE-model determinants, we identified additional determinants related to professional behaviours in promoting healthy GWG. We identified these determinants as knowledge, barriers, work-related stress, involving other professionals, health promotion and personal experience.

\section{Knowledge}

Five midwives were not aware of the IOM-guidelines or the KNOV-guidelines, nor about the general relevance of gaining less weight with a higher pre-pregnancy BMI. However, they were all aware of the association between high GWG and weight retention postpartum. Knowledge about healthy eating and PA was superficial, except for the one midwife with a special interest in these issues.

\section{Work-related stress}

Midwives experienced their work as demanding and this seemed to influence their flexibility in implementing new guidelines or looking for new ways to enhance the quality of care they provide.

The whole midwifery world is like ... well, there is so much to do ... we need to do so many things ... we don't want to add something else.

[About other midwives experiencing everything as burdensome] Everything is seen as a heavy load. And that ... if everything ... if everything that comes to you is seen as heavy ... yes, your life becomes heavy too. You cannot motivate them [other midwives] to do something with it.

\section{Barriers}

Barriers were mentioned that impede midwives from Monitoring GWG, providing DietEducation or PA-Education. Indicated were a lack of time, money and energy on the part of both midwives and pregnant women. Furthermore, midwives expressed that they lacked a Dutch guideline or practice card with easy to use information to help guide pregnant women.

You can do it in a nice way or you can make nice things. And this is a good part of midwifery. But, you know, it's always a question of time and money and ... and ... always the question of what everyone is willing to invest.

Interviewer: it needs to be feasible?

Yes. And the pregnant woman needs to go along with you. So ... sometimes it takes energy to convince her. To teach her the need.

\section{Involving other professionals}

Some midwives were active in using the expertise of other health professionals. Midwives who involved other health professionals, including dieticians, psychologists and professionals providing PA-courses for pregnant women, seemed to be more active in promoting healthy 
GWG. It seemed to stimulate them to think in a broader perspective and to take account of other professionals' point of view. They also seemed to be relieved that someone else could take care of this part of promoting healthy GWG. Being acquainted with other professionals helped midwives in working together with them. All midwives seemed to be aware of the influence they had on pregnant women, and assumed pregnant women would go to another health professional if they advised them to do so. But some midwives were reluctant to involve other professionals because they felt it had no added value.

And when you say they [pregnant women] need to go to a dietician, well, then women do that. ... But I often think, well, they [pregnant women] know what to do and what not to do, so I think the dietician can't really add anything to that.

\section{Health promotion}

In the course of the interviews the researchers gained the impression that some midwives viewed themselves as professionals who adhere to guidelines simply because they were taught to do so. This was expressed by midwives who referred to guidelines using language such as "we have to" and "they want us to", while others expressed an intrinsic motivation to help women take an important new step in their lives. The latter expressed their wish to contribute to public health in general out of a desire to help create a healthier world. They aimed to find the best way to reach their ideals and they expressed their interest in and concern about a wide variety of topics. One of the participants used a metaphor to express this, seeing herself as an ambassador of health.

Look, women go their various way in life and we hope to see them get on the right track. When they are on the right track, we don't need to do anything. They step into the right train and then they go off on their own ... the train is moving ... But some colleagues ... they even don't bring them to the station, you know ... they leave them to sort it out for themselves. And to be honest, our profession doesn't compensate us for telling women why you want them to have a healthy lifestyle. You need to go into detail, to be able to adjust your advice to their lives. The same is true for maternal distress.

While this midwife pro-actively helped women onto "the right train", another midwife tries to empower women so they can find "their own train".

Well, I think it's necessary to raise women's awareness of their own body. Most women don't know their own body. They don't know the meaning of certain physical complaints and their reactions. You know, I very often say to women: "nowadays, we seem to be pregnant on top of everything else. We work 100 percent, we are a 100 percent partner, 100 percent social contacts and we want to look good 100 percent of the time. Yes, and on top of all of that, we happen to be pregnant." I say, "that's not the way it works. In the first place you're pregnant, 
and the time that's left can be used for the rest". And that's another way of looking at things: "oh yes, I am just plain pregnant. I can't do it in the sixth gear. I'm going back to zero and then I can see what's left for the other things". I think it's a turning point for women when they realize: "well, I'm pregnant, I am simply pregnant. And that is what my life is about. It's not about finishing my work or about my partner having enough sex or that I am there for my friends. No, it's about me, being pregnant. Taking good care of myself and of my baby". I encourage them to trust themselves. I ask them: "What does your intuition tell you, your own answer?" And they say, "Yes, I think I need to do this or that". Well, that is the right answer. Just do it.

I think, it is just ... so good when you, being a woman, think: "I am good the way I am. And I can do it".

A third midwife expressed her attitude toward health promotion from the perspective of the woman.

By not saying anything, I think you then stimulate unhealthy behaviours. "Yeah, the midwife didn't say anything, so it must be ok". That. No, you are an advisor; you are there to help women, to promote their health.

Although these three midwives expressed their attitude toward health promotion in different ways, all three combined promoting healthy GWG with their general attitude toward their profession. Midwives who did not pro-actively look for solutions to problems they encountered were nonetheless willing to promote healthy GWG if they were provided clear guidelines on GWG.

The practice card you are going to develop ... yes, I am impatiently waiting for you to do that.

\section{Personal experience}

Midwives expressed the similarity between their clients' experiences and their own experiences with weight gain during their pregnancies. If they had an issue with diet during pregnancy themselves, it seemed that they expected it to be an issue for women in their practice, and vice versa.

I say to them, "don't forget to eat". Because that's my own experience. When you have two kids ... you forget to eat. You need to eat sitting at the table, you need to eat properly, because as a young mother, I think you tend not to do that. 
If you are constantly concerned with your weight and always thinking about what you eat ... I think you see pregnant women differently ... yes, maybe that is ... maybe that's why it's not such a big deal for me.

\subsection{Discussion}

We were able to identify behaviours to promote healthy GWG (Monitoring GWG, DietEducation and PA-Education) and the ASE-determinants related to these behaviours. Midwives were willing to perform monitoring and educational behaviours. Some already performed these behaviours and others expressed their willingness to do so if advised by trustworthy sources. We found that what midwives described as their intention corresponded with their behaviour. Midwives had moderately positive attitudes towards Monitoring GWG and Diet-Education. Their attitude to PA-Education was less positive, but their perception of the importance and effectiveness of PA-Education seemed to change during the interview, suggesting that awareness of the need to engage in PA was an important determinant for their behaviour. Concerning social influence, midwives were not always aware of what other midwives do to promote healthy GWG, nor of what pregnant women expect them to do. Midwives experienced sufficient self-efficacy. In the study of Heslehurst et al., midwives were not confident in their ability to discuss weight-related issues ${ }^{227}$. Our sample experienced problems with discussing weight-related issues as well, but in the past. After implementation of the NVOG guidelines on obesity and pregnancy, they quickly discovered that they had to discuss weight as a reason to refer obese women to an obstetrician. While this was difficult in the past, they no longer had difficulties with discussing how to regulate GWG.

In addition to the ASE-determinants, we distinguished a number of determinants that likely play an important role in influencing midwives' behaviours in promoting healthy GWG. Midwives in our study were not aware of the risks of excessive or too little GWG. They did not know that weight gain goals vary per pre-pregnancy BMI group, and they filled the gap in their knowledge with personal beliefs and experiences. Knowledge has been recognized as an important determinant for behaviour in behavioural models. ${ }^{191}$ The same is true for barriers, which are more often mentioned in behavioural models ${ }^{228}$ and which were present in our findings. In our case the most important barriers seemed to be a lack of available guidelines and a lack of time. Work-related stress seemed to hinder the behaviours in promoting healthy GWG as well. Some of the participants expressed that they found it difficult to balance the demands of the job with the rewards of the job and were on the verge of a burn-out. Wiegers et al. studied work diaries kept by Dutch primary care midwives and estimated that a full-time workload of a primary care midwife translates to 87 full cases per year. ${ }^{223}$ This means that the required 105 cases for a full-time salary amounts to $120 \%$ of a full-time work week. The part of the job experienced as most fulfilling, the client-related work, decreased in 2008 (67\%) as compared to 2001-2004 (73\%). ${ }^{223}$ Lindqvist et al. conducted a qualitative study on midwives' experiences in counselling pregnant women on PA in Sweden. ${ }^{205}$ They noted that the Swedish National Board of Health and Welfare recommended that counselling about lifestyle changes, 
such as increasing PA, be performed in short sessions of 10-15 minutes and in sessions of 30 minutes for more challenging cases. The Swedish midwives who were interviewed considered this to be a rather short amount of time, in which they felt the pressure of time constraint. ${ }^{205}$ In the Netherlands, by comparison, the first visit is scheduled for twenty minutes (excluding an echo and counselling on screening for trisomy 21), while subsequent visits are scheduled for ten minutes each. ${ }^{221}$ If we want Dutch midwives to spend more time on health promotion, including promoting healthy GWG, decreasing midwives' workload could be an important way to facilitate and support their work in this respect.

Involving other professionals could be related to midwives' sense of urgency about the problem of unhealthy GWG, unhealthy diet and lack of PA. When midwives are unaware of the health risks related to these problems, they may tend not to seek help from another professional. Midwives aware of the problem of unhealthy GWG, on the other hand, seek solutions to the questions that arise on the topic. Referral to other professionals could be a way to provide solutions.

Midwives who saw themselves as an important professional in a significant life-changing event seemed to pay more attention to healthy lifestyle for the long-term benefit of the whole family and were more willing to involve other care providers. They were "of course" promoting healthy GWG and healthy lifestyles. Other midwives, however, were more reluctant in promoting healthy GWG and it did not occur to them to be pro-active in looking for solutions to common problems they encountered (such as weight-related problems). We found this variation in how midwives define their role in this regard to be comparable to Walsh and Devane, who noticed that some midwives see themselves as a partner in health, helping the client to realize her own strength, while others see their role as a medical guard of pregnancy and birth, and focus primarily on risks and safety. ${ }^{229}$ We also compared our findings with the findings of a Swedish study in which antenatal appointments of midwives were filmed and analyzed. ${ }^{230}$ The authors described two perspectives of antenatal care. The first view is focused on the physical process of birth and the latter on the process of becoming parents, including the psychological and social circumstances in addition to the physical. ${ }^{230}$ Being focused on the physical process in the study of Olsson et al. could be related to the reluctant attitude we encountered in our study. When guidelines explain and prescribe why and how midwives need to change their practice, the physically oriented/reluctant midwives follow the guidelines. It could be that midwives who focus on the total process of becoming parents also focus on health promotion in general, including promoting healthy GWG, healthy diet and healthy PA. Our findings from the interviews together with the literature cited above would seem to confirm our hypothesis that midwives' attitude and midwives' activity in health promotion are associated with behaviours in promoting healthy GWG.

In our study we found midwives who felt that advice that had helped them in their personal situation would be helpful to their clients as well. Having personal weight problems has been mentioned in other research as a determinant for midwives' behaviour in promoting healthy GWG. ${ }^{227}$ 
So far, this study has provided only qualitative evidence for midwives' behaviour. Quantitative studies are indicated in order to reach more sound conclusions about midwives' behaviours and the determinants related to these behaviours. Although our sample consists of only a limited number of participants, we believe we were able to identify important determinants of behaviour, which was the aim of this study. This is because the sample was diverse and informed us about the various views of the midwives. The major determinants were mentioned several times across all interviews.

This information can be used to create an adapted model for midwives' behaviour to promote healthy GWG. We recommend that intention be removed, and that other determinants be added, including barriers, work-related stress, involving other health professionals, health promotion, and personal experience with weight-related problems, as these determinants have been revealed as important for primary care midwives.

\subsection{Conclusion}

We conducted a qualitative study on midwives' behaviours in promoting healthy GWG, with the guidance of the ASE-model. Midwives confirmed the determinants provided by this model and enabled us to identify several additional determinants. Quantitative research is needed to measure the extent of the determinants associated with midwives' behaviours in promoting healthy GWG. 


\section{Chapter 6}

\section{Dutch midwives' behaviour and determinants in promoting healthy gestational weight gain, phase 2: a quantitative approach}

Astrid Merkx, Marlein Ausems, Luc Budé, Raymond de Vries, Marianne J. Nieuwenhuijze

International Journal of Childbirth 2015; 5(3):139-53 


\section{Background}

Unhealthy gestational weight gain (GWG) contributes to long term obesity in women and their offspring. The aim of this study is to quantify midwives' behaviour in promoting healthy GWG and to identify the most important determinants related to this behaviour.

\section{Methods}

A survey based on the Attitude-Social influence-self-Efficacy (ASE) -model and prior qualitative research was conducted among 112 Dutch practicing midwives.

\section{Results}

Midwives were moderate active in monitoring GWG, diet-education and less active in physical activity-education. Regression analysis showed that efforts to promote healthy GWG were influenced by a number of determinants, including attitudes, self-efficacy, social influence, the involvement of other health workers, health promotion and barriers.

\section{Practice Implications}

The identified behavioural determinants provide insight into ways to stimulate midwives to promote healthy GWG. 


\subsection{Introduction}

High gestational weight gain (GWG) (i.e. weight gain above the Institute of Medicine (IOM)guidelines) is associated with obesity in the long term for both mothers and their offspring. ${ }^{2,3}$ ${ }^{5,6}$ Healthy GWG yields better obstetric outcomes compared with too high or too low GWG. ${ }^{6}$ The percentage of women in high-income countries who gain weight within the IOMguidelines varies from $21.6 \%$ to $48.7 \% .{ }^{41,126}$ The incidence of women in the Netherlands who gain weight below (19\%), within (44\%) and above the IOM-guidelines (38\%), ${ }^{44}$ also demonstrates a clear need to focus on healthy GWG in order to improve the health prospects of mothers and babies. Interventions using GWG-monitoring, diet-education and education about physical activity (PA) can influence women's GWG. ${ }^{145,216}$ Prenatal care providers are in a position to provide these interventions. The problem of too high GWG has not yet been effectively addressed, however. ${ }^{44,45}$ Exploration of how to develop successful interventions for promoting GWG is necessary. One of the routes to increasing the number of women who gain a healthy amount of weight during pregnancy is to stimulate and facilitate care providers to promote healthy GWG during prenatal care. The question arises how prenatal care providers, who have limited time, can be facilitated to direct their attention in an effective way to promoting healthy GWG.

In order to influence prenatal care providers' behaviour it is crucial that the intervention be tailored to their practices. ${ }^{62}$ It is necessary, therefore, to have a clear view of what their current behaviour with regard to promoting healthy GWG is, and what determinants encourage or discourage their behaviour.

Midwives working in primary care are the main prenatal care providers in the Netherlands. Close to $85 \%$ of all pregnant women begin their prenatal care with a midwife. ${ }^{143}$ Fifty-four percent of all pregnant women continue to receive prenatal care from a midwife at the end of pregnancy. ${ }^{143}$ The Dutch midwifery system is described elsewhere. ${ }^{204}$ Qualitative studies of midwives have examined their educational needs and explored determinants related to their behaviour in promoting healthy GWG. ${ }^{204,227}$ Midwives revealed their need for training and education by acknowledging a lack of relevant skills and knowledge. Moreover, midwives had varying attitudes toward promoting healthy GWG, and their engagement in health promotion in general seemed to influence their daily practice in promoting healthy GWG. ${ }^{204,227}$ The aim of this paper is to quantify midwives' behaviour in promoting GWG and to identify the most important determinants related to this behaviour. By doing so we provide information that can be used for developing an evidence-based intervention to effectively promote healthy GWG, tailored to the practice of prenatal care providers.

\subsection{Methods and materials}

\subsubsection{Model}

Drawing on previous intervention studies on promoting healthy GWG, we divided the general behaviour "promoting healthy GWG" into three sub-behaviours, including "Monitoring 
GWG", "Diet-Education" and "PA-Education" (Fig. 6.1). We used the ASE-model as a basis. ${ }^{189}$ We used qualitative studies to adapt the general ASE-model to build our hypothesized model. ${ }^{204,227}$ Attitude, social influence, self-efficacy and barriers are related to a specific behaviour (grey boxes in Figure 6.1). The attitudes related to Diet-Education (such as "I believe Diet-Education is important"), e.g., can differ from attitudes toward Monitoring GWG ("I believe Monitoring GWG is important").

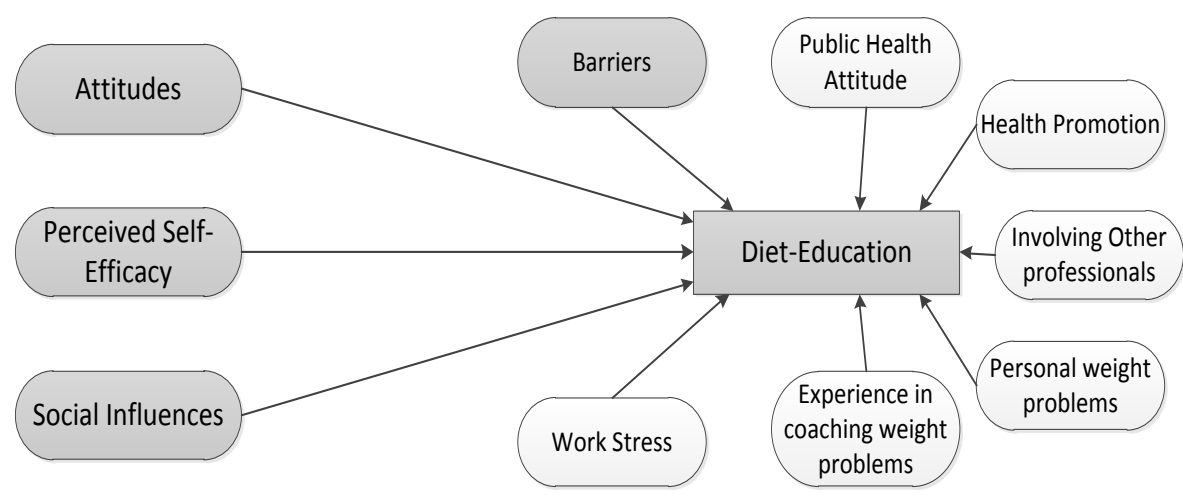

Figure 6.1 Model for Diet-Education used for the regression models.

Grey boxes are specific for a given behaviour; here for example, the behaviour is Diet-Education. The boxes containing Attitudes, Perceived Self-Efficacy, Social Influences and Barriers are related to Diet-Education. Where the behaviour is "monitoring gestational weight gain (GWG)", the grey boxes are also related to monitoring GWG. The white boxes remain the same for all behaviours.

\subsubsection{Procedure}

We conducted a survey among primary care midwives, who were recruited in June and July 2012. We sent an email to 164 midwifery practices affiliated with our institute, and placed two general announcements in the digitally distributed newsletter sent to all members of the Dutch Royal College of Midwives (98\% of all primary care midwives). Midwives were invited to participate and a link to the online survey was provided in the invitation.

The study was entered into the Dutch Trial Register under number TC 3543. Due to the noninvasive character of the study, the Research Ethics Committee of Atrium-Orbis Zuyd confirmed that ethical approval was not necessary.

\subsubsection{The questionnaire}

The questionnaire included 156 items divided into six categories:

1. Midwife characteristics (4 items): age (in years), education (three years midwifery education, four years midwifery education, university level), work-experience (in years) and workload (number of cases per year). 
2. Practice characteristics (16 items): working environment (solo practice, own practice in group practice, or as an employee), specific aspects of care (time spent on intake, number of postpartum care visits, etc.), presence and tasks of a practice assistant.

3. Monitoring GWG (64 items): We asked midwives about weighing in four separate groups (average body type, appearing too thin, appearing too heavy and women with a history of weight problems). We posed these items for the first visit and for subsequent visits. We asked two questions about discussing GWG. We also asked how BMI was defined (self-reported or measured weight and length).

We asked participants how often they actually engaged in a specific behaviour when they 'discussed GWG'. To construct these items we used literature on behavioural change techniques, goal-setting, education and motivational interviewing (Box 6.1). ${ }^{231}$

Box 6.1 Behavioural components of "discussing GWG"

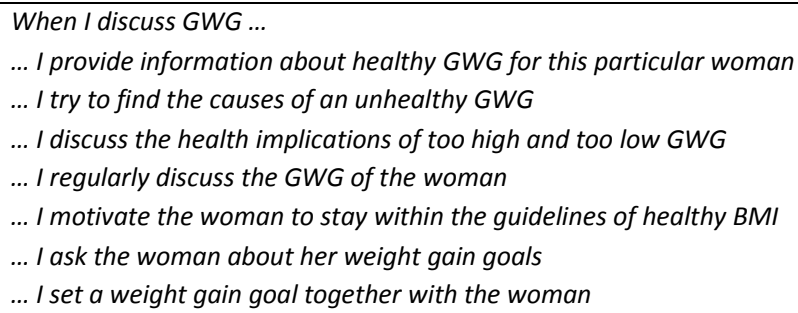

We also inquired about Monitoring GWG related attitudes (e.g., "for me, discussing GWG is important"), social influences (e.g., "my clients expect me to discuss GWG with them"), perceived self-efficacy (e.g., "I am good at discussing GWG with my clients"), barriers (e.g., "I lack guidelines"), knowledge and time spent on discussing GWG.

4. Diet-Education (24 items): Items included performed behaviour (advice to follow a healthy diet, discussing diet, discussing resistance to conversations about diet), related attitudes (importance and effectiveness of behaviour), social influence ("my clients expect me to discuss their diet"), perceived self-efficacy of the behaviour and barriers (e.g., "I lack guidelines to promote healthy eating").

5. PA-Education (27 items): Items included performed behaviour (advice on the norms for healthy PA, discussing complaints of over-exertion), related attitudes (importance and effectiveness of behaviour), social influence, perceived self-efficacy of the behaviour and barriers (e.g., "I lack time to inform women about healthy PA").

6. Additional determinants ( 21 items): We asked about the involvement of various health professionals for achieving a healthy GWG (including general practitioner, obstetrician, dietician, physiotherapist, non-regular therapist, psychologist and special pregnancy course), attitude toward public health (one item), work-related stress (three items), personal 
experience with weight problems (one item) and experience in coaching others about weight issues (one item). In addition we developed a scale about regular behaviour with respect to general health promotion, derived from the World Health Organization (WHO) definition of health and health promotion as shown in Box 6.2. ${ }^{232}$

Box 6.2 components of determinant "Health Promotion"

l ask my client about her physical well-being.

l ask my clients about their emotional coping.

l ask my clients about their social support.

I ask my clients about their sleep.

I confront my clients with their unhealthy lifestyle.

I stimulate my clients to have a healthy lifestyle.

Twelve items were open-ended, 23 multiple-choice, and the remaining (121) could be answered on a seven-point Likert scale, ranging from 1 (totally disagree/never) to 7 (totally agree/always). The questionnaire was pretested among seven practicing midwives and seven midwifery lecturers using cognitive interviewing for clarity, phrasing and sequence. ${ }^{233}$ This iterative process was repeated until content validity seemed to be reached. The questionnaire was subsequently pilot-tested among ten final-year midwifery students, who were asked for their feedback afterwards. This led to minor adjustments in wording.

\subsubsection{Analyses}

One item with more than $10 \%$ missing values was excluded (knowledge-question on how much weight a women should gain in the first thirteen weeks of pregnancy). Crude data was used for descriptive analysis. For inferential statistics, missing data on items missing less than $10 \%$ were imputed with sample means.

We had three separate outcomes: GWG-Monitoring, Diet-Education and PA-Education. We computed GWG-Monitoring by calculating the mean of 1 ) the two moments of weighing (first visit and subsequent visit across all four types of women) and 2) the two "discussing GWG"items. Diet-Education and PA-Education were computed by calculating the means of items measuring that specific behaviour. We used Cronbach's alpha to check the internal consistency of the items in these three composite outcome variables. Dummy variables were constructed for categorical items with more than two values (Education and Working Environment). Pearson's correlation coefficients were used to measure the association between each of the dependent variables (GWG-Monitoring, Diet-Education and PAEducation) and the variables hypothesized as determinants of the three behaviours (Figure 6.1). The initial models used in our regression analyses for these three dependent variables included: 1) ASE-determinants (Attitude, Social-Influences, Perceived-Self-Efficacy), 2) additional determinants (Barriers, Work-Stress, Involving Others, Health Promotion, PublicHealth-Attitude, Experience-Coaching, and Personal-Weight-Problems) that were significantly correlated with the behaviour concerned and 3) a limited number of covariates (Age, Working Environment, Education, Workload). ${ }^{234}$ We used a manual backward model selection strategy 
for our regression analyses. At each step the weakest determinant, based on $p$-value, was removed. In the final model a two-sided $\alpha \leq 0.05$ was used as criterion for inclusion of variables. We present only the final models.

\subsection{Results}

The questionnaire was completed by 118 midwives. We cannot determine the response rate because we do not know how many midwives took note of the announcement. We excluded the surveys completed by six midwives working in a secondary care capacity in hospitals. Characteristics of the remaining 112 participants and their practices are shown in Tables 6.1 and 6.2. Personal characteristics of our sample were fairly comparable to a recent study on the quality and provision of Dutch midwifery care. ${ }^{46}$

Table 6.1. Characteristics of Participants ( $n=112)$

\begin{tabular}{llc}
\hline Personal & Categories & Percentages \\
\hline Education & 3 years midwifery & 26.8 \\
& 4 years midwifery (Bachelor) & 67.0 \\
& University degree (MSc or PhD) & 6.3
\end{tabular}

Age (years)

Working Experience (years)

\begin{tabular}{cc}
\hline Mean (sd) & Range \\
\hline $36.12(10.0)$ & $22-62$ \\
$11.77(9.4)$ & $0.5-41$ \\
$85.0(35.0)$ & $40-380$ \\
\hline
\end{tabular}

Workload (full cases per participant per year)

Abbreviations: MSc, Masters of Science; PhD, Doctor of Philosophy; sd, standard deviation

Table 6.2 Characteristics of Practices $(n=112)$

\begin{tabular}{|c|c|c|c|c|}
\hline Practice & Categories & n (\%) & & \\
\hline \multirow[t]{3}{*}{ Working Environment } & own practice in group & $83(74.1)$ & & \\
\hline & solo practice & $11(9.8)$ & & \\
\hline & working as employee & $18(16.1)$ & & \\
\hline \multicolumn{5}{|l|}{ five or more visits during } \\
\hline \multicolumn{2}{|l|}{ first week postpartum } & $48(42.9)$ & & \\
\hline \multicolumn{2}{|c|}{ Working with Practice Assistant } & $72(64.3)$ & & \\
\hline & & & Mean (sd) & Range \\
\hline \multicolumn{3}{|c|}{ Time spent on first check-up; echo and screening excluded (minutes) } & $41.7(11.4)^{\mathrm{a}}$ & $20-75$ \\
\hline \multicolumn{3}{|c|}{ Time spent on subsequent check-ups (minutes) } & $14.1(3.3)^{b}$ & $10-30$ \\
\hline \multicolumn{3}{|c|}{ Time spent on extra pregnancy information meetings (minutes) } & $55.7(75.4)^{c}$ & $0-360$ \\
\hline \multicolumn{3}{|c|}{ Number of midwives in practice $(\mathrm{n})$} & $3.87(1.8)^{\mathrm{a}}$ & $1-11$ \\
\hline \multicolumn{3}{|c|}{ Women in practice coming for six week postpartum check-up (\%) } & $62.95(27.7)^{a}$ & $2-100$ \\
\hline
\end{tabular}

Abbreviations: $n$, number; sd, standard deviation

${ }^{a} n=111^{b} n=110^{c} n=109$ 


\subsubsection{Monitoring gestational weight gain (GWG) and determinants}

Table 6.3 shows that most midwives weighed and discussed healthy GWG to a moderately positive extent (mean $5.5 \mathrm{sd} 1.3$ ). Nearly all midwives who weighed women during the first visit also weighed them during subsequent visits. Differences in weighing among the four subgroups (low, normal, high BMI, women with weight problems in history) were marginal. With regard to the definition of "discussing GWG", the highest scores were given to "providing information about healthy GWG" and "trying to find reasons for why GWG was too high or too low" (Table 6.4). Lowest scores were given on "asking women about their personal goals for GWG" and "setting of GWG goals with the clients in a shared decision". All respondents calculated the BMI of all of their clients. The mean estimated time spent on GWG over the course of prenatal care was 12.7 minutes (range 1 to 60) per woman. Fortythree percent of the respondents answered the four (remaining) items on GWG-knowledge correctly.

On average, attitudes toward GWG-Monitoring were positive. Midwives thought it was more important than effective to discuss GWG with women. Higher scores were given on importance to discussing GWG with women with a high BMI than with women with a normal BMI. Midwives' self-efficacy expectations toward GWG-Monitoring were high and on average midwives experienced positive social influences, mostly from clients and from the Dutch Organization of Midwives. The most important barrier was a lack of guidelines. Attitudes, social Influence and barriers were significantly correlated with GWG-Monitoring; self-efficacy was not.

Table 6.3 Characteristics of Gestational Weight Gain (GWG)-Monitoring and related determinants ( $n=112)$

\begin{tabular}{|c|c|c|c|}
\hline $\begin{array}{l}\text { sub-items of variable } \\
\text { Likert scale ( } 1=\text { totally disagree, } 7=\text { totally agree) }\end{array}$ & $\begin{array}{l}\text { Mean }(s d) \text { of } \\
\text { sub-items }\end{array}$ & $\begin{array}{l}\text { Composite } \\
\text { variable }\end{array}$ & $\begin{array}{l}\text { Mean (sd } \\
\text { Cronbach } \\
r\end{array}$ \\
\hline $\begin{array}{l}\text { During the first visit I weigh women ... } \\
\ldots \text { with an average body type } \\
\text {... who appear too thin } \\
\text {... who appear over-weight } \\
\ldots \text { with weight problems in history }\end{array}$ & $\begin{array}{l}5.7(2.1) \\
\alpha=0.974\end{array}$ & & \\
\hline $\begin{array}{l}\text { During subsequent visits I weigh women ... } \\
\ldots \text { with a healthy BMI } \\
\ldots \text { who have a low BMI } \\
\text {... who have a high BMI } \\
\text {... with weight problems in history }\end{array}$ & $\begin{array}{l}5.3(2.2) \\
\alpha=0.977\end{array}$ & $\begin{array}{l}\text { GWG- } \\
\text { Monitoring }\end{array}$ & $\begin{array}{l}5.5(1.3) \\
\alpha=0.777\end{array}$ \\
\hline $\begin{array}{l}\text { I help women with a healthy BMI toward a healthy GWG } \\
\text { I help women with a high BMI toward a healthy GWG }\end{array}$ & $\begin{array}{l}5.5(1.4) \\
5.5(1.3)\end{array}$ & & \\
\hline
\end{tabular}




\begin{tabular}{|c|c|c|c|}
\hline \multicolumn{4}{|l|}{ I believe it is important to discuss GWG with ... } \\
\hline ... women with a normal BMI & $4.9(1.5)$ & \multirow{14}{*}{$\begin{array}{l}\text { GWG-Mo- } \\
\text { nitoring- } \\
\text { Attitude }\end{array}$} & \multirow{14}{*}{$\begin{array}{l}5.3(0.8) \\
\alpha=0.860 \\
\text { r with GWG- } \\
\text { Monitoring } \\
=0.428^{* * *}\end{array}$} \\
\hline ... women with a high BMI & $6.5(0.8)$ & & \\
\hline ... women with a low BMI & $6.2(1.1)$ & & \\
\hline ... women who gain too much & $6.3(1.0)$ & & \\
\hline ... women who gain too little & $5.5(1.4)$ & & \\
\hline I believe it is effective to discuss GWG with ... & & & \\
\hline ... women with a normal BMI & $4.0(1.5)$ & & \\
\hline ... women with a high BMI & $4.6(1.3)$ & & \\
\hline ... women with a low BMI & $4.4(1.3)$ & & \\
\hline ... women who gain too much & $4.8(1.3)$ & & \\
\hline ... women who gain too little & $4.2(1.2)$ & & \\
\hline \multicolumn{2}{|l|}{ Therefore I do not pay attention to GWG } & & \\
\hline I believe education on GWG belongs in prenatal care. & $6.1(1.0)^{\mathrm{a}}$ & & \\
\hline $\begin{array}{l}\text { When I discuss GWG with women, it disrupts the bond } \\
\text { between me and the woman. }\end{array}$ & $1.9(1.4)^{\mathrm{a}}$ & & \\
\hline \multicolumn{4}{|c|}{ Suppose I want to discuss GWG, I feel competent in women who ... } \\
\hline ... have a normal BMI & $5.9(0.9)$ & \multirow{6}{*}{$\begin{array}{l}\text { GWG-Mo- } \\
\text { nitoring-Self- } \\
\text { Efficacy }\end{array}$} & \multirow{6}{*}{$\begin{array}{l}5.5(0.7) \\
\alpha=0.828 \\
r \text { with GWG- } \\
\text { Monitoring = } \\
0.064 \text { (ns) }\end{array}$} \\
\hline ... have a high BMI & $5.3(1.2)$ & & \\
\hline ... have a low BMI & $5.4(1.2)$ & & \\
\hline ... gain too much & $5.5(1.0)$ & & \\
\hline ... gain too little & $5.5(1.1)$ & & \\
\hline I believe I know enough to help women with their GWG & $5.6(0.9)$ & & \\
\hline \multicolumn{4}{|c|}{ I believe these persons think it is important that I help women toward a healthy } \\
\hline \multicolumn{4}{|l|}{ GWG ... } \\
\hline ... pregnant women & $5.4(1.1)$ & \multirow{8}{*}{$\begin{array}{l}\text { GWG-Mo- } \\
\text { nitoring- } \\
\text { Social- } \\
\text { Influence }\end{array}$} & \multirow{8}{*}{$\begin{array}{l}4.7(1.1) \\
\alpha=0.899 \\
r \text { with GWG- } \\
\text { Monitoring = } \\
0.338^{* * *}\end{array}$} \\
\hline ... partners of pregnant women & $4.5(1.3)$ & & \\
\hline ... other midwives in my practice & $4.8(1.5)$ & & \\
\hline ... other midwives in my neighbourhood & $4.2(1.6)$ & & \\
\hline ... the Dutch Professional Organization of Midwives & $5.2(1.4)$ & & \\
\hline ... obstetricians & $4.4(1.6)$ & & \\
\hline ... general practitioners & $4.3(1.6)$ & & \\
\hline $\begin{array}{l}\text { I believe my clients expect me to help them toward a } \\
\text { healthy GWG }\end{array}$ & $4.8(1.4)$ & & \\
\hline \multicolumn{4}{|c|}{ I experience the following barriers in helping women toward a healthy GWG ... } \\
\hline ... lack of time & $3.7(2.1)$ & \multirow{6}{*}{$\begin{array}{l}\text { GWG-Mo- } \\
\text { nitoring- } \\
\text { Barriers }\end{array}$} & \multirow{6}{*}{$\begin{array}{l}3.7(1.4) \\
\alpha=0.825 \\
r \text { with GWG- } \\
\text { Monitoring = } \\
-0.328^{* * *}\end{array}$} \\
\hline ... lack of good guidelines & $4.3(1.8)$ & & \\
\hline ... lack of materials & $4.1(1.9)$ & & \\
\hline ... lack of knowledge & $3.3(1.8)$ & & \\
\hline ... lack of skills & $2.8(1.7)$ & & \\
\hline ... lack of mutual agreements with other professionals & $3.7(1.8)$ & & \\
\hline
\end{tabular}

\footnotetext{
$* * * \mathrm{p}<0.001$
}

Abbreviations: BMI, Body Mass Index; GWG, Gestational Weight Gain; ns, not significant

a Excluded from GWG-Monitoring-Attitude due to low Cronbach's $\alpha$ 
Table 6.4 Additional information on Gestational Weight Gain (GWG)-Monitoring ( $n=112$ )

\begin{tabular}{|c|c|c|c|}
\hline Subject & Content of & $\%(n)$ & Mean (sd) \\
\hline Definition & When I discuss GWG ... (Likert scale 1=never to 7=always) & & \\
\hline of & ... I provide information about healthy GWG for this woman & & $5.4(1.5)$ \\
\hline discussing & ... I try to find causes of unhealthy GWG & & $5.2(1.6)$ \\
\hline \multirow[t]{5}{*}{ GWG } & ... I discuss the implications of too high and too low GWG & & $4.9(1.6)$ \\
\hline & ... I discuss the GWG of the woman regularly & & $4.8(1.6)$ \\
\hline & ... I motivate the woman to stay within the guidelines of healthy BMI & & $4.8(1.7)$ \\
\hline & ... I ask the woman about her weight gain goals & & $1.8(1.2)$ \\
\hline & ... I set a weight gain goal together with the woman & & $2.0(1.5)$ \\
\hline \multirow[t]{6}{*}{ Knowledge } & A healthy BMI is between 25 and $30 \mathrm{~kg} / \mathrm{m}^{2}$ (false) & $88.4(99)$ & \\
\hline & A healthy GWG depends on the BMI (true) & $81.3(91)$ & \\
\hline & For a woman with a normal BMI 20 kilo GWG is normal (false) & $65.2(73)$ & \\
\hline & A healthy GWG protects against weight retention postpartum & 92.0 & \\
\hline & (true) & $(103)$ & \\
\hline & all answers correct & $42.9(48)$ & \\
\hline \multirow[t]{9}{*}{$B M I$} & I measure BMI of all women (yes) & $100(112)$ & \\
\hline & I use for weight ... & & \\
\hline & ... self-reported weight before pregnancy & $42.9(48)$ & \\
\hline & ... weight of woman during first visit & $55.4(62)$ & \\
\hline & ... self-reported last weight & $1.8(2)$ & \\
\hline & I use for length ... & & \\
\hline & ... self-reported length & $77.7(87)$ & \\
\hline & ... length of woman measured in first visit & $13.4(15)$ & \\
\hline & ... length in passport & $8.9(10)$ & \\
\hline \multirow[t]{2}{*}{ Time } & Time spent on GWG during entire course of prenatal care (in & & 12.7 (11.0) range \\
\hline & 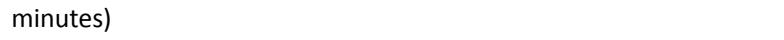 & & $1-60$ \\
\hline
\end{tabular}

Abbreviations: BMI=Body Mass Index; GWG=Gestational Weight Gain; n, number; sd, standard deviation

\subsubsection{Diet-Education and determinants}

The mean score for Diet-Education was 5.2 (sd 1.1) (Table 6.5). Within the determinant Diet-

Education-Attitude, importance scored higher than effectiveness. On average, midwives perceived their self-efficacy with respect to Diet-Education as sufficient and assumed that - to a certain extent - pregnant women expected them to educate them about diet. The highest score among the barriers was for lack of time. All ASE-determinants were significantly correlated with Diet-Education. 
Table 6.5 Characteristics of Diet-Education and related determinants ( $n=112$ )

\begin{tabular}{|c|c|c|c|}
\hline $\begin{array}{l}\text { sub-items of variable } \\
\text { Likert scale, (1=totally disagree, } 7=\text { totally agree) }\end{array}$ & Mean (sd) & $\begin{array}{l}\text { Composite } \\
\text { variable }\end{array}$ & $\begin{array}{l}\text { Mean (sd); } \\
\text { Cronbach's } \alpha ; \\
r\end{array}$ \\
\hline $\begin{array}{l}\text { I help women gain or maintain a healthy diet } \\
\text { I discuss diet with women with a high BMI } \\
\text { If a woman does not want to talk about her diet, I discuss } \\
\text { with her the reason for her resistance }\end{array}$ & $\begin{array}{l}5.6(1.1) \\
5.4(1.1) \\
5.0(1.4)\end{array}$ & $\begin{array}{l}\text { Diet- } \\
\text { Education }\end{array}$ & $\begin{array}{l}5.2(1.1) \\
\alpha=0.681\end{array}$ \\
\hline $\begin{array}{l}\text { I believe most people have a healthy diet } \\
\text { I believe I can prevent gestational diabetes by discussing } \\
\text { women's diet }\end{array}$ & $\begin{array}{l}3.9(1.2) \\
4.8(1.5)\end{array}$ & \multirow{7}{*}{$\begin{array}{l}\text { Diet- } \\
\text { Education- } \\
\text { Attitude }\end{array}$} & \multirow{7}{*}{$\begin{array}{l}5.1(1.0) \\
\alpha=0.649 \\
r \text { with Diet- } \\
\text { Education = } \\
0.822^{* * *}\end{array}$} \\
\hline $\begin{array}{l}\text { I believe helping someone toward a healthy diet is time } \\
\text { consuming }\end{array}$ & $5.2(1.4)$ & & \\
\hline $\begin{array}{l}\text { I believe the subject of diet is so important that I want to } \\
\text { spend extra time on it }\end{array}$ & $5.0(1.4)$ & & \\
\hline $\begin{array}{l}\text { I believe it is important to discuss the diet of a woman with a } \\
\text { high BMI }\end{array}$ & $5.1(1.1)$ & & \\
\hline $\begin{array}{l}\text { I believe it is effective to discuss the diet of a woman with a } \\
\text { high BMI }\end{array}$ & $4.9(1.4)$ & & \\
\hline $\begin{array}{l}\text { I believe it is important to discuss resistance with a woman } \\
\text { who does not want to talk about her diet }\end{array}$ & $5.5(1.2)$ & & \\
\hline $\begin{array}{l}\text { I believe it is effective to discuss resistance with a woman } \\
\text { who does not want to talk about her diet }\end{array}$ & $4.6(1.5)$ & & \\
\hline $\begin{array}{l}\text { I believe I am good in discussing the diet of a woman with a } \\
\text { high BMI } \\
\text { I believe I am good in discussing resistance with a woman } \\
\text { who does not want to talk about her diet }\end{array}$ & $5.4(1.1)$ & $\begin{array}{l}\text { Diet- } \\
\text { Education- } \\
\text { Self-Efficacy }\end{array}$ & $\begin{array}{l}5.1(1.1) \\
\alpha=0.778 \\
r \text { with Diet- } \\
\text { Education = } \\
0.639 * * *\end{array}$ \\
\hline Women expect me to talk about their diet & $5.1(1.2)$ & $\begin{array}{l}\text { Diet- } \\
\text { Education- } \\
\text { Social- } \\
\text { Influence }\end{array}$ & $\begin{array}{l}\text { r with Diet- } \\
\text { Education }= \\
0.350 * * *\end{array}$ \\
\hline \multicolumn{4}{|l|}{$\begin{array}{l}\text { I experience the following barriers in helping women toward } \\
\text { a healthy diet ... }\end{array}$} \\
\hline ... lack of time & $4.8(1.8)$ & \multirow{6}{*}{$\begin{array}{l}\text { Diet- } \\
\text { Education- } \\
\text { barriers }\end{array}$} & \multirow{6}{*}{$\begin{array}{l}3.6(1.3) \\
\alpha=0.838 \\
r \text { with Diet- } \\
\text { Education = - } \\
0.212^{*}\end{array}$} \\
\hline ... lack of good guidelines & $3.9(1.8)$ & & \\
\hline ... lack of materials & $3.6(1.8)$ & & \\
\hline ... lack of knowledge & $3.1(1.6)$ & & \\
\hline ... lack of skills & $2.8(1.6)$ & & \\
\hline ... lack of mutual agreements with other professionals & $3.5(1.7)$ & & \\
\hline
\end{tabular}

$* \mathrm{p}<0.05{ }^{* * *} \mathrm{p}<0.001$

Abbreviations: BMI, Body Mass Index; n, number; r, correlation; sd, standard deviation

\subsubsection{Physical activity Education and determinants}

The mean score for PA-Education was 4.1 (sd 1.3) (Table 6.6). On average, midwives discussed physical signs of over-exertion more often than they educated pregnant women about healthy PA, and they were more positive towards discussing physical signs of over-exertion than towards education about healthy PA. Additionally, midwives perceived their self-efficacy 
positively and did not believe that pregnant women expected much information from them about healthy PA. Concerning barriers, highest scores were on lack of guidelines, lack of time and lack of materials. All ASE-determinants were significantly correlated with PA-Education.

Table 6.6 Characteristics of physical activity (PA)-Education and related determinants $(n=112)$

\begin{tabular}{|c|c|c|c|}
\hline $\begin{array}{l}\text { sub-items of variable } \\
\text { Likert scale, ( } 1=\text { totally disagree, } 7=\text { totally agree) }\end{array}$ & Mean (sd) & $\begin{array}{l}\text { Composit } \\
\text { e variable }\end{array}$ & $\begin{array}{l}\text { Mean (sd); } \\
\text { Cronbach' } \\
s \alpha ; r\end{array}$ \\
\hline I help women toward healthy physical activity & $4.8(1.4)$ & \multirow{5}{*}{$\begin{array}{l}\text { PA- } \\
\text { Education }\end{array}$} & \multirow{5}{*}{$\begin{array}{l}4.1(1.3) \\
\alpha=0.839\end{array}$} \\
\hline I ask women what their activity pattern is & $3.0(1.9)$ & & \\
\hline I explain what healthy physical activity is & $3.0(1.9)$ & & \\
\hline I explain the benefits of healthy physical activity for mother and baby & $4.2(1.8)$ & & \\
\hline I discuss the signals of overexertion & $5.3(1.6)$ & & \\
\hline I believe it is important to ask the woman about her activity pattern & $4.3(1.6)$ & \multirow{10}{*}{$\begin{array}{l}P A- \\
\text { Education } \\
\text {-Attitude }\end{array}$} & \multirow{10}{*}{$\begin{array}{l}4.7(1.1) \\
\alpha=0.891 \\
r \text { with } P A- \\
\text { Education } \\
=0.725^{* * *}\end{array}$} \\
\hline I believe it is effective to ask the woman about her activity pattern & $4.1(1.5)$ & & \\
\hline $\begin{array}{l}\text { I believe it is important to explain to women what healthy physical } \\
\text { activity is }\end{array}$ & $4.3(1.6)$ & & \\
\hline $\begin{array}{l}\text { I believe it is effective to explain to women what healthy physical activity } \\
\text { is }\end{array}$ & $4.0(1.6)$ & & \\
\hline $\begin{array}{l}\text { I believe it is important to explain the benefits of healthy physical activity } \\
\text { for mother and baby }\end{array}$ & $5.1(1.3)$ & & \\
\hline $\begin{array}{l}\text { I believe it is effective to explain the benefits of healthy physical activity } \\
\text { for mother and baby }\end{array}$ & $4.9(1.3)$ & & \\
\hline I believe it is important to discuss signals of overexertion & $5.5(1.3)$ & & \\
\hline I believe it is effective to discuss signals of overexertion & $5.5(1.3)$ & & \\
\hline I believe discussing physical activity is time consuming & $4.4(1.4)$ & & \\
\hline $\begin{array}{l}\text { I believe discussing healthy physical activity is important enough to spend } \\
\text { my time on }\end{array}$ & $4.4(1.4)$ & & \\
\hline I believe I am good in asking the woman about her activity pattern & $5.9(1.1)$ & \multirow{4}{*}{$\begin{array}{l}\text { PA- } \\
\text { Education } \\
\text {-Self- } \\
\text { Efficacy }\end{array}$} & \\
\hline $\begin{array}{l}\text { I believe I am good in explaining to women what healthy physical activity } \\
\text { is }\end{array}$ & $5.2(1.5)$ & & $5.3(1.1)$ \\
\hline $\begin{array}{l}\text { I believe I am good in explaining the benefits for mother and baby of } \\
\text { healthy physical activity }\end{array}$ & $5.0(1.5)$ & & $\begin{array}{l}\alpha=0.842 \\
r \text { with } P A-\end{array}$ \\
\hline I believe I am good in discussing signals of overexertion & $5.3(1.4)$ & & $\begin{array}{l}\text { Education } \\
=0.509 * * *\end{array}$ \\
\hline $\begin{array}{l}\text { I believe the women I work with expect me to help them toward healthy } \\
\text { physical activity }\end{array}$ & $4.1(1.4)$ & $\begin{array}{l}\text { PA- } \\
\text { Education } \\
\text {-Social- } \\
\text { Influence }\end{array}$ & $\begin{array}{l}\text { r with } P A- \\
\text { Education } \\
=0.467^{* * *}\end{array}$ \\
\hline
\end{tabular}

I experience the following barriers in helping women toward healthy physical activity ...

... lack of time

... lack of good guidelines

... lack of materials

... lack of knowledge

$\left.\begin{array}{l}4.4(1.9) \\ 4.5(1.8) \\ 4.4(1.8) \\ 3.5(1.7) \\ 3.3(1.6) \\ 3.9(1.9)\end{array}\right\}$

$4.0(1.3)$

PA$\alpha=0.858$

... lack of skills

... lack of mutual agreements with other professionals

$* \mathrm{p}<0.05 * * * \mathrm{p}<0.001$

Abbreviations: $n$, number; $r$, correlation; sd, standard deviation; PA, Physical Activity 


\subsubsection{Associations between characteristics and the three behaviours}

We found associations between midwives' behaviours and the midwives' practice characteristics (Table 6.7). Positive associations were seen between Time spent for the First Check-up with Diet-Education and with PA-Education. Furthermore, Percentage of Women coming for Six Week Post-partum Check-up was positively associated with Diet-Education.

Table 6.7 Characteristics and their relation to the three behaviours $(n=112)$

\begin{tabular}{|c|c|}
\hline & Pearson's correlation ( $r$ ) \\
\hline \multirow[t]{3}{*}{ Time spent on first check-up; echo and screening excluded (minutes) $^{a}$} & r with GWGM ns \\
\hline & r with DE $0.388^{* * *}$ \\
\hline & r with PAE $0.192^{*}$ \\
\hline \multirow[t]{3}{*}{ Time spent on subsequent check-ups (minutes) ${ }^{b}$} & r with GWGM ns \\
\hline & r with DE ns \\
\hline & $r$ with PAE ns \\
\hline \multirow[t]{3}{*}{ 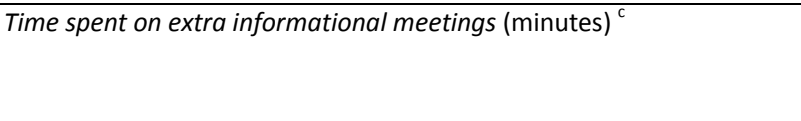 } & r with GWGM ns \\
\hline & $r$ with DE ns \\
\hline & r with PAE ns \\
\hline \multirow[t]{3}{*}{ Number of midwives in practice $(\mathrm{n})^{\text {a }}$} & r with GWGM ns \\
\hline & r with DE ns \\
\hline & $\mathrm{r}$ with PAE $0.185^{\circ}$ \\
\hline \multirow[t]{3}{*}{ Women in practice coming for check-up 6 weeks postpartum (\%) } & r with GWGM ns \\
\hline & r with DE 0.196* \\
\hline & $r$ with PAE ns \\
\hline \multirow[t]{3}{*}{ Age (years) } & r with GWGM ns \\
\hline & r with DE ns \\
\hline & $r$ with PAE ns \\
\hline \multirow[t]{3}{*}{ Work experience (years) } & r with GWGM ns \\
\hline & r with DE ns \\
\hline & $r$ with PAE ns \\
\hline \multirow[t]{3}{*}{ Workload (full cases per year) } & r with GWGM ns \\
\hline & r with DE ns \\
\hline & r with PAE ns \\
\hline
\end{tabular}

\subsubsection{Additional determinants and their associations}

Table 6.8 presents the results of the additional determinants of midwives' behaviours in promoting healthy GWG. On average, midwives experienced their work-related stress as neutral. Midwives hardly involved others concerning GWG; they most frequently called upon dieticians and least frequently upon obstetricians. Midwives had a very positive Public-HealthAttitude. The mean score for Health Promotion, representing education behaviours in six fields related to public health, was positive. Individual items in this determinant showed that midwives were more active in discussing physical wellbeing (6.1 sd 1.1) and sleep (5.5 sd 1.1) than in discussing emotional coping (4.4 sd 1.6) and social support ( $4.5 \mathrm{sd} 1.6)$. The variables 
Health Promotion and Involving Others showed positive correlations with the outcome variables.

Table 6.8. Additional determinants and their relation to the three behaviours $(n=112)$

\begin{tabular}{|c|c|c|c|}
\hline $\begin{array}{l}\text { sub-items of variable } \\
\text { Likert scale, 1=totally disagree, 7=totally agree }\end{array}$ & Mean (sd) & Variable & $\begin{array}{l}\text { Mean (sd); } \\
\text { Cronbach's } \alpha ; \\
r\end{array}$ \\
\hline $\begin{array}{l}\text { I experience enough fulfilment in my work } \\
\text { (reversed in scale) } \\
\text { I often work under great work pressure } \\
\text { My work demands a lot of energy }\end{array}$ & $\begin{array}{l}4.4(1.7) \\
5.3(1.4)\end{array}$ & Work-Stress & $\begin{array}{l}4.1(1.2) \alpha=0.602 \\
\text { r with } G W G M \mathrm{~ns} \\
\text { r with } D E \text { ns } \\
\text { r with } P A E \text { ns }\end{array}$ \\
\hline $\begin{array}{l}\text { I involve the following others in promoting a } \\
\text { healthy GWG ... } \\
\ldots \text { general practitioner } \\
\ldots \text { obstetrician } \\
\ldots \text { dietician } \\
\ldots \text { physiotherapist } \\
\ldots \text { a non-regular therapist } \\
\ldots \text { psychologist } \\
\ldots \text { a special pregnancy course }\end{array}$ & $\begin{array}{l}2.4(1.7) \\
2.0(1.5) \\
5.8(1.1) \\
2.2(1.7) \\
2.2(1.5) \\
2.6(1.7) \\
2.9(2.0)\end{array}$ & $\begin{array}{l}\text { Involve-Other } \\
\text { Professionals }\end{array}$ & $\begin{array}{l}2.9(0.8) \\
\alpha=0.758 \\
r \text { with GWGM } 0.176^{\circ} \\
\text { r with } D E \text { ns } \\
\text { r with } P A E 0.437^{* * *}\end{array}$ \\
\hline $\begin{array}{l}\text { I ask the woman about her ... } \\
\ldots \text { physical wellbeing } \\
\ldots \text { social support } \\
\text {... emotional coping } \\
\ldots \text { sleeping pattern } \\
\text {... ideas to change toward a healthier lifestyle } \\
\text { I confront women with their unhealthy behaviour }\end{array}$ & $\begin{array}{l}6.1(1.1) \\
4.5(1.6) \\
4.4(1.6) \\
5.5(1.1) \\
5.1(1.3) \\
5.2(1.1)\end{array}$ & $\begin{array}{l}\text { Health } \\
\text { Promotion }\end{array}$ & $\begin{array}{l}5.1(0.9) \\
\alpha=0.700 \\
r \text { with GWGM ns } \\
\text { r with } D E 0.455^{* * *} \\
\text { r with } P A E 0.437^{* * *}\end{array}$ \\
\hline $\begin{array}{l}\text { I think it is very important that midwives pay } \\
\text { attention to the general health of women }\end{array}$ & & $\begin{array}{l}\text { Public-Health- } \\
\text { Attitude }\end{array}$ & $\begin{array}{l}6.3(0.8) \alpha \text { na } \\
\text { r with GWGM } 0.163^{\circ} \\
\text { r with } D E 0.209^{*} \\
\text { r with } P A E 0.306^{* *}\end{array}$ \\
\hline $\begin{array}{l}\text { I have experience in helping others to } \\
\text { keep/maintain a healthy BMI }\end{array}$ & & $\begin{array}{l}\text { Experience- } \\
\text { Coaching }\end{array}$ & $\begin{array}{l}4.0(2.0) \alpha \text { na } \\
\text { r with } G W G M \text { ns } \\
\text { r with } D E 0.231^{*} \\
\text { r with } P A E \text { ns }\end{array}$ \\
\hline $\begin{array}{l}\text { I experience problems with keeping/maintaining } \\
\text { a healthy BMI myself }\end{array}$ & & $\begin{array}{l}\text { Personal- } \\
\text { Weight- } \\
\text { Problems }\end{array}$ & $\begin{array}{l}3.2(2.2) \alpha \text { na } \\
\text { r with GWGM ns } \\
\text { r with } D E \text { ns } \\
\text { r with } P A E \text { ns }\end{array}$ \\
\hline
\end{tabular}

${ }^{\circ} \mathrm{p}<0.1{ }^{*} \mathrm{p}<0.05{ }^{* *} \mathrm{p}<0.011^{* * *} \mathrm{p}<0.001$

Abbreviations: BMI, Body Mass Index; DE, Diet Education; GWG, Gestational Weight Gain; GWGM, GWG Monitoring; na, not applicable; ns, not significant; PA, Physical Activity; PAE, PA Education; sd, standard deviation; r, correlation

\subsubsection{Regression analyses}

The final models of the regression analyses for GWG-Monitoring, Diet-Education and PAEducation are presented in Table 6.9.

The first model explained $34 \%$ of the variance in GWG-Monitoring. Increases in GWGMonitoring-Attitude $(b=0.594, p=0.000)$ and $G W G$-Monitoring-Social-Influence $(b=0.212$, 
$p=0.044$ ) were associated with an increase in the score on GWG-Monitoring. Higher scores on GWG-Monitoring-barriers ( $b=-0.271, p=0.001$ ) were associated with a decrease in GWG-

Monitoring. Self-Efficacy was not significantly associated. Working Environment was a significant covariate; working in a group practice was associated with an increase in GWGMonitoring as compared to working in a solo practice $(b=-0.726, p=0.048)$ and working as an employee ( $b=-0.701, p=0.019)$.

The explained variance of Diet-Education was $76 \%$. Increases in Diet-Education-Attitude $(b=0.644, p=0.000)$, Diet-Education-Self-Efficacy $(b=0.212, p=0.001)$, Diet-Education-SocialInfluence $(b=0.105, p=0.024)$ and Health Promotion $(b=0.160, p=0.019)$ were associated with an increased Diet-Education score. Diet-Education-Barriers was not a significant determinant. Workload and Education were significant covariates; a higher workload ( $b=-0.003, p=0.028$ ) was associated with lower scores in Diet-Education and three years of education compared to four years $(b=0.252, p=0.038)$ was associated with higher scores in Diet-Education. The explained variance of $P A-E d u c a t i o n$ was $68 \%$. Increases in PA-Education-Attitudes $(b=0.522, p=0.000), P A-E d u c a t i o n-S e l f-E f f i c a c y(b=0.238, p=0.002), P A-E d u c a t i o n-S o c i a l-$ Influence $(b=0.121, p=0.044)$, Involving Others $(b=0.295, p=0.000)$, Health Promotion $(b=0.233, p=0.019)$ and a decrease in PA-Education-Barriers $(b=-0.142, p=0.014)$ were associated with an increase in $P A-E d u c a t i o n$. Confounders were not significantly associated.

Table 6.9 Final Models of Regression Analyses for gestational weight gain (GWG)-Monitoring, Diet-Education and physical activity (PA)-Education $(n=112)$

\begin{tabular}{lllll}
\hline GWG-Monitoring / $\mathbf{R}^{\mathbf{2}}=\mathbf{0 . 3 4 1}$ & $\mathbf{B}$ & $\mathrm{SE}$ & $\begin{array}{l}\text { standardized } \\
\text { coefficient }\end{array}$ & p-value \\
\hline Intercept & & \multicolumn{3}{c}{0.015} \\
GWG-Monitoring-Attitude & 2.340 & 0.942 & 0.326 & 0.000 \\
GWG-Monitoring-Social-Influence & 0.594 & 0.164 & 0.179 & 0.044 \\
GWG-Monitoring-Barriers & 0.212 & 0.104 & -0.276 & 0.001 \\
$\begin{array}{l}\text { Working Environment (ref own practice in } \\
\text { cooperation) }\end{array} \quad-0.271$ & 0.080 & & 0.048 \\
$\quad$ Solo & -0.726 & 0.363 & -0.163 & 0.019
\end{tabular}

The initial model also included: GWG-Monitoring-Self-Efficacy, Public-Health-Attitude, Midwives' Education, Involve Others, Age and Workload.

\begin{tabular}{lllll}
\hline Diet-Education / $\mathbf{R}^{\mathbf{2}}=\mathbf{0 . 7 5 8}$ & $\mathbf{B}$ & $\mathbf{S E}$ & $\begin{array}{l}\text { standardized } \\
\text { coefficient }\end{array}$ & p-value \\
\hline Intercept & & & 0.468 \\
Diet-Education-Attitude & -0.295 & 0.406 & & 0.000 \\
Diet-Education-Self-Efficacy & 0.644 & 0.073 & 0.609 & 0.001 \\
Diet-Education-Social-Influence & 0.212 & 0.064 & 0.225 & 0.024 \\
Health Promotion & 0.105 & 0.046 & 0.118 & 0.019 \\
Workload & 0.160 & 0.067 & 0.127 & 0.028 \\
Midwives' Education (ref 4 yrs) & -0.003 & 0.002 & -0.111 & 0.038 \\
$\quad$ 3 years $\quad$ university & 0.252 & 0.120 & 0.105 & 0.072
\end{tabular}

The initial model also included: Diet-Education-Barriers, Public-Health-Attitude, Experience-Coaching, Working Environment and Age. 


\begin{tabular}{lllll}
\hline PA-Education / $\mathbf{R}^{\mathbf{2}}=\mathbf{0 . 6 7 6}$ & $\mathbf{B}$ & SE & $\begin{array}{l}\text { standardized } \\
\text { coefficient }\end{array}$ & p-value \\
\hline Intercept & & & & 0.006 \\
PA-Education-Attitude & -1.610 & 0.576 & & 0.000 \\
PA-Education-Self-Efficacy & 0.522 & 0.085 & 0.436 & 0.002 \\
PA-Education-Social-Influence & 0.238 & 0.074 & 0.202 & 0.044 \\
PA-Education-Barriers & 0.121 & 0.060 & 0.131 & 0.014 \\
Involving Other Professionals & -0.142 & 0.057 & -0.143 & 0.000 \\
Health Promotion & 0.295 & 0.078 & 0.223 & 0.019
\end{tabular}

The initial model also included: Public-Health-Attitude, Midwives' Education, Working Environment, Age and Workload.

Abbreviations: GWG, Gestational Weight Gain; PA, Physical Activity

\subsection{Discussion and conclusions}

Our findings give us important information for the development of an intervention to help midwives promote a healthy GWG during prenatal care.

\subsubsection{Gestational weight gain monitoring}

Overall, midwives in this study were moderately active in GWG-Monitoring and this was more or less the same for women in all pre-pregnancy BMI-groups. Midwives had moderately positive attitudes towards GWG-Monitoring, felt confident and experienced moderate support from clients and peers. Midwives also experienced barriers, such as a lack of guidelines and a lack of materials. There is room for improvement, however. All of the ASEdeterminants except self-efficacy were significantly correlated with GWG-Monitoring, meaning that when attitudes and social influence increase and barriers diminish, GWGMonitoring is likely to increase. To increase the attitudes of midwives toward GWGMonitoring, it seems necessary to encourage midwives to look more positively at discussing GWG with pregnant women and to convince them that pregnant women expect them to discuss GWG. As self-efficacy is not a significant determinant, skills-training would not seem necessary. ${ }^{62}$ Providing guidelines with standard care norms and materials, such as growth charts, could be helpful in promoting a healthy GWG.

We saw that the estimated time spent on GWG was approximately $5 \%$ of total prenatal care time, with major differences being present. In line with obesity guidelines in the Netherlands, ${ }^{224}$ all midwives assessed BMI. This suggests that midwives are faithful to practical guidelines concerning weight issues. Introducing guidelines for GWG-monitoring, accompanied by materials and resources, could be promising in this respect. More than half of our sample lacked sufficient knowledge about GWG, which corresponds with findings from other studies. ${ }^{44,235-237}$ In the definition of discussing GWG, midwives revealed that they provided information on GWG, but rarely discussed weight gain goals together with women. In a Dutch study using video-recordings it was observed that during the first visit women were weighed, but that weight gain was barely discussed. ${ }^{46}$ Three studies revealed that accurate goal-setting and advice by healthcare professionals were positively correlated with pregnant women achieving a healthy GWG. ${ }^{59,215,238}$ We would argue that midwives need to be 
educated about the importance of discussing weight-gain goals in a shared decision-making process.

\subsubsection{Diet-Education}

On average, the midwives involved in this study were moderately active in Diet-Education. Their attitudes, social influences, perceived self-efficacy and "Health Promotion" were significantly correlated with Diet-Education. Although the variable Diet-Education-Barriers (with lack of time the important part) was not a significant determinant; Workload was, however. These findings imply that midwives could give more Diet-Education when they are convinced of its importance and effectiveness and when clients expect them to provide this education. Furthermore, midwives can be stimulated by skills training to improve their selfefficacy and by providing efficient ways to educate their clients. Midwives with higher scores on health promotion also scored higher on Diet-Education. In this respect, convincing midwives to pay more attention to their clients' health in general, including emotional coping and social support, would likely have its effect on Diet-Education as well. Additionally, since a high Workload and less Time spent on the first check-up were also significantly associated with Diet-Education, we conclude that midwives experience a lack of time to go into DietEducation. Therefore, other solutions, such as involving dieticians, should be explored as well. Midwives with three instead of four years of education were more active in Diet-Education. This could be explained by the history of Dutch midwifery education. The midwifery curriculum changed from three years to four years in $1995 .{ }^{239}$ Therefore, the group of midwives with four years of education includes recently graduated midwives who have less work experience. More experienced midwives could have learned about healthy eating during their working years. This finding may also indicate a lack in education in current Dutch midwifery programs.

\subsubsection{Physical activity-education}

Midwives in this study paid moderate attention to PA-Education. Most of the attention went to dealing with complaints and not to informing women about healthy PA. This finding is comparable to that of an earlier Dutch study, in which only $41 \%$ of participating midwives stated that they always discussed PA as a standard subject of prenatal care. ${ }^{240}$ This is difficult to interpret since we do not know the content of what was discussed. All ASE determinants were significantly associated with PA-Education, as were Barriers, Involving Others and Health Promotion. There is clearly room for improvement, by, for instance, influencing midwives' attitudes, training them in discussing healthy PA with clients and by convincing them about their clients' needs in this respect. PA-Education could improve as well when midwives have higher scores on Health Promotion and Involvement. This can be achieved by raising awareness about various aspects of health, including emotional coping and social support, and when midwives are encouraged to involve other health professionals more often. Involving other health professionals could also save time, which was an important barrier. 


\subsubsection{Reflection on the quality of this study}

To the best of our knowledge, this study is the first quantitative study that offers insights into Dutch midwives' behaviours in promoting healthy GWG. Although our sample was quite comparable to another Dutch midwifery study, selection bias must be taken into account. Midwives working as employees were underrepresented in this study. ${ }^{241}$ Because we found that midwives working in a group practice had higher levels of GWG-Monitoring, the average score of 5.5 for this outcome would likely have been lower if more participants had been employees. Furthermore, midwives who agreed to participate may have been more interested in GWG and this self-selection may have led to higher scores. Finally, we used selfreported data and did not objectively measure midwives' behaviours, which could have led to more positive scores. With these remarks in mind, we believe this study can be useful for other settings where midwives work in prenatal care.

The ASE determinants, together with the additional determinants, explained a large amount of the variance in midwife behaviours related to GWG. The explained variance in the models for Diet-Education and PA-Education was high. This could be the result of the high correlations between Diet-Education and Diet-Education-Attitudes, for example, and between $P A$-Education and PA-Education-Attitudes. Respondents may have interpreted the questions as asking for the same information.

Our questionnaire was developed for this project, and has not yet been validated. To enhance the quality of the questionnaire, we used the adapted ASE-model as the theoretical basis for the item development and we used a thorough process in developing the questionnaire, including involvement of representatives of the study population.

\subsubsection{Conclusion}

Our study revealed that midwives were fairly active in GWG-monitoring in pregnant women in all pre-pregnancy BMI-groups and that their attitudes, social influence and barriers were related to GWG-Monitoring. Midwives in a group practice were more active in GWGmonitoring than employed midwives. Midwives were also more active in Diet-Education then in PA-Education. Attitudes, perceived self-efficacy, social influences and health promotion were significantly related to these educational behaviours. Additionally, midwives who more often involved other health professionals were also more active in PA-Education. DietEducation was hampered by time constraints. The barriers, including time constraint and lack of guidelines, hampered PA-Education. Midwives who received their midwifery education longer ago were more active in Diet-Education than their more recently educated peers. The determinants found to be significantly associated with midwives' behaviours in promoting healthy GWG can be influenced and can therefore be used to develop an intervention for midwives. An effective intervention could help to reduce the burden caused by obesity, one of today's major health problems. Attitudes, e.g., can be enhanced by convincing midwives of the importance and the benefits of Monitoring GWG, Diet-Education and PA-Education, and educating them about the role midwives can play in improving these behaviours. Social influences can be improved by educating midwives about the expectations 
of clients and by setting the norm within the professional organization. Self-efficacy can be improved by skills training. Barriers can be diminished by providing guidelines, resources and by inventing ways to reduce the time constraint midwives experience. Midwives need to be encouraged to involve other health professionals. This will decrease the demand on midwives' time and allow pregnant women to benefit from the expertise of these professionals. Stimulating midwives to pay attention to all aspects of health, including emotional coping and social support, will likely have a positive effect on Diet-Education and PA-Education as well. The highly positive attitude of midwives towards the importance of their public health role can be used as a motivator.

Future research is needed to explore strategies that can be used to improve midwives' GWGMonitoring, Diet-Education and PA-Education and to explore strategies for influencing the cooperation of midwives with other health professionals. The education of midwives in DietEducation in current midwifery curricula should also receive attention. 


\title{
Chapter 7
}

\section{“Come On!" Using Intervention Mapping to help healthy pregnant women achieve healthy weight gain.}

\author{
Astrid Merkx, Marlein Ausems, Raymond de Vries, \\ Marianne J. Nieuwenhuijze
}

Public Health Nutrition 2017; 1-15

doi:10.1017/S1368980017000271 


\section{Background}

Gaining too much or too little weight in pregnancy (according to the Institute Of Medicine IOM)-guidelines) negatively affects both mother and child, but many women find it difficult to manage their gestational weight gain (GWG). In this paper we describe the use of "Intervention Mapping" (IM) to design "Come On!" an intervention to promote adequate GWG among healthy pregnant women.

\section{Methods}

We used the six steps of IM: (I) needs assessment; (II) formulation of change objectives; (III) selection of theory-based methods and practical strategies; (IV) development of an intervention program; ( $\mathrm{V}$ ) development of an adoption and implementation plan; and (VI) development of an evaluation plan. A consortium of users and related professionals guided us in the process of development.

\section{Results}

As a result of the Needs Assessment two goals for the intervention were formulated: 1 . Helping healthy pregnant women to stay within the IOM-guidelines for GWG and 2. Getting midwives to adequately support the efforts of healthy pregnant women to gain weight within the IOM-guidelines. To reach these goals, change objectives and determinants influencing the change objectives were formulated. Theories used were the Trans Theoretical Model, the Social Cognitive Theory and the Elaboration Likelihood Model.

\section{Conclusions}

Practical strategies to use the theories were the foundation for the development of "Come on!", a comprehensive program that included: a tailored internet program for pregnant women, training for midwives, an information card for midwives, and a scheduled discussion between the midwife and the pregnant woman during pregnancy. The program was pretested and evaluated in an effect study. 


\subsection{Introduction}

Women who gain too much or too little weight during pregnancy are more likely to experience a variety of health problems during their pregnancies and in their later lives. Healthy gestational weight gain (GWG) yields the best obstetric outcomes and improves the long-term outcomes for the health and weight of mothers and their babies. ${ }^{6,13}$ In 2009, the Institute of Medicine (IOM) published revised guidelines for weight gain during pregnancy, providing recommendations for GWG based on a woman's pre-pregnancy Body Mass Index (BMI). According to these guidelines, women who begin their pregnancy being underweight (BMI less than $18.5 \mathrm{~kg} / \mathrm{m}^{2}$ ) should gain $12.5-18 \mathrm{~kg}$ during their pregnancy; normal weight women (BMI of $18.5-24.9 \mathrm{~kg} / \mathrm{m}^{2}$ ) should gain $11.5-16 \mathrm{~kg}$; women who are overweight (BMI of $25-29.9 \mathrm{~kg} / \mathrm{m}^{2}$ ) should gain 7-11.5 kg; and obese women (BMI of $30 \mathrm{~kg} / \mathrm{m}^{2}$ and above) should gain 5-9 kg. ${ }^{13}$ The percentage of women in high-income countries who gain weight within these recommendations varies from $18.9 \%$ to $51.9 \%,{ }^{2,41-43}$ demonstrating a clear need to focus on healthy GWG to improve the health prospects of mothers and babies. Several interventions for promoting healthy GWG have been studied. ${ }^{63,67,72,78,145,207,216,242-244}$ Reviews of these studies show these interventions to have varied effects, depending in large part on their target group (i.e., a general population or a specific risk group). The majority of GWG studies targets specific groups, such as overweight or obese women, or women with hypertension or gestational diabetes. Interventions adopting a dietary approach, increasing physical activity (PA), and setting weight gain goals proved to be most effective at reaching healthy GWG. ${ }^{216}$

Interventions to encourage appropriate GWG fit well with the midwifery model of care. ${ }^{24-248}$ A recent review of midwifery in the UK underscored the relationship between public health and midwifery, ${ }^{210}$ emphasizing the importance of public health interventions during pregnancy and the postnatal period. Community midwives are in a unique position to improve the health of young families because they have regular and frequent contact with pregnant women. In the Netherlands $85 \%$ of the pregnant women begin care with community midwives and more than $50 \%$ continue their pregnancy under guidance of the midwife. ${ }^{143}$ Community midwives are authorized to care for healthy pregnant women, a group that stands to benefit from the prevention of weight related disorders.

The theoretical and methodological tools used in health psychology can increase the effectiveness of health-related interventions. ${ }^{62,63,249}$ However, as Hill and colleagues found in their review it is very difficult to identify the theoretical assumptions of existing interventions designed to promote healthy GWG. ${ }^{243}$ In this paper we provide insights into the theoretical assumptions used in GWG interventions by describing the development of our "Come On!" intervention promoting healthy GWG among healthy pregnant women. Our use of "Intervention Mapping" (IM) -a systematic approach for the development of interventions based on established theory and empirical data- makes explicit the theories we used to design our program. ${ }^{62} \mathrm{IM}$ also recognizes that individual behaviour is influenced by factors in the 
environment, i.e., individual, family, social network, organizations, communities, and society. $^{62}$

\subsection{Methods}

IM consists of six steps: (I) needs assessment (NA); (II) formulation of change objectives; (III) selection of theory-based methods and practical strategies; (IV) development of intervention program; (V) development of an adoption and implementation plan; and (VI) development of an evaluation plan.

\subsubsection{Step I Needs assessment}

IM starts with the identification of the health problem, its behavioural factors and their associated individual and environmental determinants. The aim of this first step is to establish the relevant target groups and program outcomes.

In our case, the NA included an analysis of the literature on existing interventions for achieving a healthy GWG. We also analysed the literature on the determinants and correlates of GWG in pregnant women, including the role of predefined behavioural (i.e., PA and dietary behaviour) and environmental risk factors.

In addition, we conducted a quantitative survey among healthy pregnant woman to investigate the percentages of women reaching healthy GWG and to assess the relationship between, among other factors, diet and PA and reaching a healthy GWG. Furthermore, we scheduled individual interviews with community midwives to identify their behaviours with respect to promoting healthy GWG and the determinants of their behaviours. The interview protocol was based on the Attitude-Social Influence-Self-Efficacy (ASE)-model, ${ }^{249}$ a model

useful in explaining or changing a variety of behaviours. ${ }^{250,251}$ We used the information from the interviews and literature to construct our study model and questionnaire for the quantitative survey to measure midwives' self-reported behaviours and determinants. During the NA we established a project Consortium including: several midwives practicing in primary care, a dietician, physiotherapist, psychologist working with young mothers, employee of Dutch Organization for Midwives (KNOV), and health educators. During the NA, the project team met three times, to discuss the research findings, to contribute to the decision making process on the selection of target groups and to advise on the outcomes of the behavioural program. Between the meetings, we consulted with individual project members as needed.

\subsubsection{Step II Formulation of change objectives}

In this second IM step, program outcomes were subdivided into performance objectives. Performance objectives are related to sub-behaviours that must be accomplished by the target groups in order to achieve the program outcomes. By linking the performance objectives with relevant behavioural determinants, the general formulations of the performance objectives were translated into very specific change objectives. The Consortium was also involved in step II. Members offered advice about the relevance and changeability of 
the selected behavioural determinants and approved the formulation and selection of change objectives.

\subsubsection{Step III Selection of theory-based methods and practical strategies}

In Step III we identified and selected the theoretical methods, i.e., general techniques or processes for influencing changes in behavioural determinants of the target groups. To do so, the change objectives were organized per determinant. Subsequently, methods were matched to the determinants. Methods were mainly selected from the summary of theoretical methods provided by Bartholomew et al. ${ }^{62}$ We organised a brainstorm session with the Consortium in order to select the practical strategies to be used, identifying the specific techniques for employing theoretical methods in ways that fit our intervention population and the context in which our intervention will be conducted. We also relied on our literature review of existing interventions for information on feasible working mechanisms.

\subsubsection{Step IV Development of intervention program}

In step IV we combined information from previous steps, which allowed us to operationalise the theoretical methods and develop the practical applications required to accomplish the change objectives. We decided upon program scope and sequence and designed scripts and documents needed for the production of the program. During this process we pre-tested our materials among members of the target groups, including Consortium members and pregnant women.

\subsubsection{Step V Development of an adoption and implementation plan}

The fifth step focused on the planning of the adoption and implementation of the intervention. The Consortium meetings provided important information about the factors that would impede and enhance implementation in midwifery practices.

\subsubsection{Step VI Development of an evaluation plan}

In this last step we developed a plan to evaluate the program effectiveness and the quality of intervention. Our measurement instruments for evaluating program effects were based on the instruments used in the NA (step I) and on instruments for process evaluation described by Steckler et al. ${ }^{252}$

\subsection{Results}

\subsubsection{Step I Needs assessment}

In the literature, unhealthy GWG is described as both serious and widespread. The majority of published studies focused on risk groups, such as obese pregnant women. As noted above, the percentage of women in high-income countries who gain weight within the IOMguidelines varied from $18.9 \%$ to $51.9 \%{ }^{2,41-43}$ In the Netherlands at the time of the study, the incidence of women who gained weight below, within, and above the IOM-guidelines was 
respectively $18.8-33.4 \%, 39.9-43.8 \%$ and $26.7-37.6 \% .{ }^{44,45}$ For neonates, GWG below the guidelines was associated with prematurity and babies too small for their gestational age. ${ }^{122}$ GWG above the guidelines was associated with, among other things, low five minute Apgar scores, seizures, hypoglycaemia, polycythaemia, meconium aspiration syndrome, large for gestational age. ${ }^{145}$ For women, GWG above the guidelines was associated with pregnancyinduced hypertension, gestational diabetes mellitus, pre-eclampsia, caesarean delivery, postpartum weight retention, and long-term obesity. ${ }^{145,253}$ A number of studies found that diet and PA are not the only mediators of healthy GWG: ${ }^{254,255}$ the behaviour of health professionals also plays an important role. ${ }^{256,257}$ Midwives, as the providers of regular checkups during pregnancy, are important players in the process of reaching healthy GWG.

Our cross-sectional survey among 455 healthy Dutch pregnant women showed that GWG within the guidelines occurred in $42.4 \%$ of the women, $13.8 \%$ of the women gained too little and $43.9 \%$ too much. ${ }^{188}$ There was no significant correlation between GWG and prepregnancy BMI, diet, or motivation to engage in healthy PA. ${ }^{188}$ GWG below the IOMguidelines, compared to within, was seen more often in women who reported more sleep shortage. GWG above the IOM-guidelines (compared to within) was seen less often in Dutch than in non-Dutch women, in women who maintained their PA level, and in non-smoking women compared with women who stopped smoking. ${ }^{188}$ The mean weekly vegetable consumption was 953 grams (sd 447 grams). Vegetable-, Fruit- and Fish-norm (resp. $\geq 200$ grams per day; $\geq 2$ pieces per day; $\geq$ twice during the last week) was met in respectively $13.6 \%, 47.2 \%$ and $6.8 \% .{ }^{188}$ Nevertheless, the dietary behaviour of our sample was better than that of non-pregnant women of comparable ages in the Netherlands, ${ }^{129}$ indicating that pregnant women do improve their diet during pregnancy. However, none of the measured dietary behaviours were significantly associated with healthy GWG. ${ }^{188}$ More than half of the women reported a decline in PA during pregnancy and the self-reported mean pre-pregnancy PA was moderately active. A decline in PA was associated with GWG above the guidelines. Our qualitative study of six community midwives showed that midwives did monitor GWG (weighing and discussing GWG), offered education about diet, and, to a lesser degree, offered education about healthy PA. ${ }^{204}$ Behavioural determinants, originating from the ASE model, ${ }^{258}$ were confirmed and other relevant themes, including midwives' perception on their role in health promotion were added to our hypothetical model explaining midwives' behaviour in respect to promoting healthy GWG. ${ }^{204}$

Our quantitative survey study included 112 practicing community midwives. ${ }^{202}$ We found that midwives considered measuring weight, discussing GWG, education about healthy diet, and, to a lesser extent, education about healthy PA to be part of their practice. ${ }^{202}$ Midwives also agreed that discussion about public health issues (i.e., women's health) was very important and they regularly discussed health promotion issues (e.g., physical wellbeing, social support, emotional coping) with their clients. Midwives offered more GWG-monitoring when they had more positive attitudes towards it, experienced more supportive social influences concerning monitoring GWG, and had fewer barriers to GWG-monitoring. ${ }^{202}$ Education about diet and PA were more likely among midwives with more positive attitudes towards it, higher reported 
self-efficacy, more social influences supporting education on diet and PA, and greater activity in health promotion. ${ }^{202}$

The literature on existing interventions aiming at healthy GWG revealed that most interventions were developed in the USA and Europe, half of the interventions focused on a single theme (e.g., on diet or PA), the other half combined diet, PA and attention to weight. A meta-analysis showed that interventions focused on diet for obese women resulted in a mean difference of $-8.41 \mathrm{~kg}(95 \% \mathrm{Cl}-10.49,-6.34 \mathrm{~kg})$, interventions focused on PA resulted in a mean difference of $-0.83 \mathrm{~kg}(95 \% \mathrm{Cl}-1.47,-0.19 \mathrm{~kg})$ and multiple content interventions had no significant effects. Secondary outcomes (e.g., change in diet) were inconsistent. We found only one study of healthy pregnant women. ${ }^{259}$

As a result of our NA, and including the advice of the Consortium, we decided that our intervention should target two groups: (1) healthy pregnant women and (2) community midwives.

For each target group, a behavioural objective was formulated. Our intervention was designed to:

Help healthy pregnant women to stay within the IOM-guidelines; and Get midwives to adequately support the efforts of healthy pregnant women to gain weight within the IOM-guidelines.

\subsubsection{Step II Formulation of change objectives}

Table 7.1 shows the behavioural objectives translated into performance objectives (PO's), providing answers to the question "What do participants of the program need to do to succeed in the recommended health-related behaviour?"

Table 7.1 Performance objectives for pregnant women and midwives

\begin{tabular}{l} 
Help healthy pregnant women to stay within the IOM-guidelines \\
\hline PO1 Pregnant women determine their BMI \\
\hline PO2 Pregnant women monitor their GWG \\
\hline PO3 Pregnant women select ways to accomplish healthy GWG \\
\hline PO4 Pregnant women follow midwives' advice about healthy GWG \\
\hline $\begin{array}{l}\text { PO5 Pregnant women follow midwives' advice about referral to dietician, psychologist and/or an } \\
\text { exercise program }\end{array}$ \\
\hline $\begin{array}{l}\text { Get midwives to adequately support the efforts of healthy pregnant women to gain weight } \\
\text { within the IOM-guidelines. }\end{array}$ \\
$\begin{array}{l}\text { PO1 Midwives facilitate information supply about healthy GWG to all pregnant women in their first } \\
\text { trimester }\end{array}$
\end{tabular}


PO2 At a gestational age of about 16 weeks, midwives adapt their care concerning healthy GWG to individual needs of pregnant women

PO3 Midwives support pregnant women with monitoring and appraisal of their weight alteration during pregnancy

PO4 Midwives identify clients who are at greater risk for unhealthy GWG

PO5 Midwives empower those clients who are at greater risk for inappropriate GWG in reaching an healthy GWG

P06 Midwives identify pregnant women who need special care that is beyond the capabilities of midwives.

PO7 Midwives refer pregnant women, if necessary, to other healthcare professionals, e.g., a dietician

PO8 In case of referral of pregnant women to other healthcare professionals, midwives remain in contact with these professionals

PO9 In case of referral of pregnant women to other healthcare professionals, midwives support the care of those professionals

Abbreviations: BMI, Body Mass Index; GWG, Gestational weight gain; IOM, Institute of Medicine; PO, performance objective

We identified important and changeable behavioural determinants based on our literature review, ${ }^{259}$ the surveys of pregnant women and midwives, ${ }^{188,202,204}$ existing literature about determinants of (un)healthy GWG, and feedback from the Consortium.

The determinants of pregnant women's behaviour included awareness of the importance of healthy diet and sufficient PA in relation with their GWG and their (baby's) health; ${ }^{260}$ knowledge of: their own height and weight, ${ }^{135}$ calculation of their BMI and GWG recommendations, ${ }^{57}$ negative consequences of too low and too high GWG, ${ }^{188,256,261}$ positive consequences of healthy $\mathrm{GWG}^{188}$ and the positive influence of a healthy diet and healthy PA on GWG; ${ }^{135,188,262,263}$ attitudes towards a healthy GWG, ${ }^{188,264,265}$ a healthy diet and healthy PA, and following the advice of midwives; ${ }^{44,140,188,257,266-268}$ social influence of family members such as partners and mothers (information aroused during Consortium meetings) and midwives; ${ }^{188,235,256,257}$ self-efficacy expectations/skills with regard to assessing one's BMI, ${ }^{188}$ monitoring $\mathrm{GWG}^{140,188}$ healthy diet and healthy $\mathrm{PA},{ }^{140,188}$ discussing exceeding IOM recommendations with the midwife, resisting well-meant advice from friends, family members; barriers like no scale at home (information aroused during consortium meetings); pregnancy related changes in diet preferences, ${ }^{262}$ morning sickness and other physical complaints, ${ }^{269}$ and the combination of pregnancy with care for other young children. ${ }^{269}$ The determinants of midwife behaviour included awareness of their role in promoting healthy diet and PA, ${ }^{202,204}$ knowledge, including consequences, of healthy GWG, diet and PA, of cutoff points of healthy GWG, of diet and PA advice, ${ }^{202,204,256,266}$ attitudes towards encouraging healthy GWG, healthy diet and PA, ${ }^{202}$ perceived social influences from clients, clients" 
partners, colleagues, the Royal Dutch Organization of Midwives, obstetricians ${ }^{202}$ and from established guidelines, ${ }^{270}$ self-efficacy expectations/ skills towards tailoring their care to individual needs of women during the course of pregnancy, ${ }^{202}$ barriers including lack of time and lack of guidelines. ${ }^{202}$

Table 7.2 and 7.3 (at the end of this thesis) show the matrices of change objectives that linked objectives and determinants, based on our performance objectives and selected determinants. Each change objective specified what participants need to learn to accomplish the performance objective. For instance, table 7.2 shows a cell in which the performance objective "pregnant women determine their BMI" is linked with the determinant "knowledge". The question used to address this cell is: "What knowledge do pregnant women need to determine their BMI"? Answers to this question create the change objectives "Describe height and weight" and "Describe the calculation of BMI".

\subsubsection{Step III Selection of theory-based methods and practical strategies}

Tables 7.4 and 7.5 show the theory-based methods and practical strategies we chose to accomplish the change objectives for pregnant women and midwives.

Pregnant women

As we described above, we focused on pregnant women's awareness, knowledge, attitudes, social influences, self-efficacy expectations and barriers regarding weight gain within the IOM-guidelines. Two models provided us with a theoretical foundation for motivation change: the Trans Theoretical Model (TTM) ${ }^{271}$ and Social Cognitive Theory (SCT). ${ }^{272}$ TTM conceives a change as a process involving progress through five stages, starting with pre-contemplation, and then moving to contemplation, preparation, action, with final arrival at the maintenance stage. For behavioural change purposes, the first stage needs to focus on raising one's awareness, followed by providing knowledge and arguments in the second phase. During the preparation phase one needs to acquire practical information about behavioural change recognizing the value of social approval for strengthening intention to change. A critical feature of the action phase is the provision of information about the threats to the newly acquired behaviour. ${ }^{271}$ SCT identifies the essential elements for behavioural change, including: person- behaviour- environment interaction, behavioural capability (knowledge and skills), observational learning, reinforcements, expectations and self-efficacy. ${ }^{272}$ Drawing on these theories we identified methods for change: Conscious raising (TTM), Tailoring (TTM), Individualization (TTM), Active learning (SCT), Self-re-evaluation (TTM), Modelling (SCT), Guided practice (SCT), Verbal persuasion (SCT) and Facilitation (SCT). In addition we drew on Goal Setting Theory (GST) ${ }^{273}$ to motivate change in self-efficacy expectations.

We next developed practical strategies to apply these methods of change. For instance, computer tailoring was used to provide personalised information aimed at changes in awareness, attitudes, social influences, and self-efficacy expectations. The tailoring program included a self-monitoring device at different time points during pregnancy with visual feedback showing gaining below, within or above IOM-guidelines. According to Bartholomew et al., ${ }^{62}$ tailoring will be effective if there is a clear link between characteristics of the person 
and the messages that address those characteristics. We created short films to provide women with information aimed at changing social influences, self-efficacy expectations, and the perception of barriers. In creating the videos we selected those role models that pregnant women could easily identify with. Regular face-to-face communication between the midwife and pregnant woman was intended to reinforce the client's awareness, attitudes, social influences, self-efficacy expectations and to reduce the influence of barriers (Table 7.4).

Table 7.4 Methods and strategies per determinant for pregnant women

\begin{tabular}{|c|c|c|c|c|}
\hline Determinant & $\begin{array}{l}\text { Change } \\
\text { objectives }\end{array}$ & Method & Theory & Strategy \\
\hline Awareness & $\begin{array}{l}\text { aw2a, } \\
\text { aw3a, } \\
\text { aw4a, } \\
\text { aw5a }\end{array}$ & $\begin{array}{l}\text { Tailoring } \\
\text { Using imagery } \\
\text { Providing cues } \\
\text { Chunking }\end{array}$ & $\begin{array}{l}\mathrm{TTM}^{271} \\
\mathrm{SCT}^{272}\end{array}$ & $\begin{array}{l}\text { Personal communication with midwife. } \\
\text { Tailor-build web based program with information } \\
\text { about specialized professionals combined with visual } \\
\text { representation of pregnant woman's individual GWG } \\
\text { compared to healthy GWG }\end{array}$ \\
\hline Knowledge & $\begin{array}{l}\text { k1a, k1b, } \\
k 2 a, k 2 b, \\
k 2 c, k 2 d, \\
k 2 e, k 2 f, \\
k 3 a, k 3 b, \\
k 3 c, k 3 d, \\
k 3 e, k 3 f, \\
k 4 a\end{array}$ & $\begin{array}{l}\text { Tailoring } \\
\text { Active learning, } \\
\text { Chunking, } \\
\text { Using imagery }\end{array}$ & $\begin{array}{l}\mathrm{TTM}^{271} \\
\mathrm{SCT}^{272}\end{array}$ & $\begin{array}{l}\text { Personal communication with midwife. } \\
\text { Tailor-build web-based program (dosed information } \\
\text { on individual BMI, on healthy GWG tailored on } \\
\text { participant pre-pregnancy BMI, tailored information } \\
\text { about healthy diet, PA, general information about a } \\
\text { relaxed lifestyle, risks/ advantages of (un)healthy } \\
\text { GWG) } \\
\text { Active participation in monitoring own BMI, GWG }\end{array}$ \\
\hline Attitudes & $\begin{array}{l}\text { a1a, a2a, } \\
\text { a2b, a2c, } \\
\text { a2d, a2e, } \\
\text { a3a, a3b, } \\
\text { a3c, a3d, } \\
\text { a3e, a3f, } \\
\text { a4a, a5a }\end{array}$ & $\begin{array}{l}\text { Self- } \\
\text { reevaluation } \\
\text { Individualization } \\
\text { Tailoring }\end{array}$ & $\begin{array}{l}\mathrm{TTM}^{274} \\
\mathrm{SCT}^{272}\end{array}$ & $\begin{array}{l}\text { Personal communication with midwife, about e.g., } \\
\text { healthy GWG, healthy diet, PA, monitoring weight, } \\
\text { referral to relevant health care professionals } \\
\text { Tailor-build web based program: when gaining too } \\
\text { much or too little weight an advice comes up to } \\
\text { discuss this freely with midwife, or to consult a } \\
\text { specialist } \\
\text { A short film in the tailor-build web based program: a } \\
\text { role model provides information and arguments on } \\
\text { individual healthy GWG tailored on participant pre- } \\
\text { pregnancy BMI, PA; with general information about } \\
\text { healthy diet and relaxed lifestyle, risks/ advantages of } \\
\text { (un)healthy GWG }\end{array}$ \\
\hline $\begin{array}{l}\text { Social } \\
\text { influences }\end{array}$ & $\begin{array}{l}\text { si1a, si2a, } \\
\text { si2b, si3a, } \\
\text { si3b, si3c, } \\
\text { si3d, si3e, } \\
\text { si4a, si5a }\end{array}$ & $\begin{array}{l}\text { Information } \\
\text { about other's } \\
\text { approval }\end{array}$ & $\begin{array}{l}\mathrm{TTM}^{271} \\
\mathrm{SCT}^{272}\end{array}$ & $\begin{array}{l}\text { Regularly personal communication with midwife. } \\
\text { Tailor-build web-based program: stimulating } \\
\text { messages to discuss GWG problems with midwives, } \\
\text { with suggestions e.g., how to cope with children } \\
\text { disliking vegetables. } \\
\text { Tailor-build web based program: reinforcing } \\
\text { messages from the pregnant woman's midwife. } \\
\text { A quote on midwives website about the cooperation } \\
\text { with specialized health professionals } \\
\text { Tailor-build web based program: in case of unhealthy } \\
\text { GWG, an advice comes up to discuss this freely with } \\
\text { midwife, to consult a specialist. }\end{array}$ \\
\hline
\end{tabular}


Tailor-build web based program: Video's with peers talking about their visit to a dietician / exercise program

\begin{tabular}{|c|c|c|c|c|}
\hline $\begin{array}{l}\text { Self-efficacy/ } \\
\text { skills }\end{array}$ & $\begin{array}{l}\text { se1a, se2a, } \\
\text { se3a, se3b, } \\
\text { se3c, se3d, } \\
\text { se3e, se3f, } \\
\text { se } 4 a\end{array}$ & $\begin{array}{l}\text { Goal setting } \\
\text { Verbal } \\
\text { persuasion } \\
\text { Guided practice }\end{array}$ & $\begin{array}{l}\mathrm{SCT}^{272} \\
\mathrm{GST}^{273}\end{array}$ & $\begin{array}{l}\text { Personal communication with midwife. } \\
\text { Tailor-build web based program with FAQ's about } \\
\text { personal weight gain goal followed by feedback, short } \\
\text { films with e.g., demonstration of peers buying/ } \\
\text { preparing vegetables, exercising, and peers discussing } \\
\text { their coping with too high GWG }\end{array}$ \\
\hline Barriers & $\begin{array}{l}\text { b2a, b3a, } \\
\text { b3b, b3c, } \\
\text { b3d, b4a, } \\
\text { b4b }\end{array}$ & Facilitation & $\mathrm{SCT}^{272}$ & $\begin{array}{l}\text { Personal communication with midwife. } \\
\text { Tailor-build web based program with visual } \\
\text { representation of barriers (e.g., physical complaints, } \\
\text { care for offspring) and information about how to } \\
\text { overcome them }\end{array}$ \\
\hline
\end{tabular}

Abbreviations BMI, Body Mass Index; FAQ, Frequently asked questions; GST, Goal Setting Theory; GWG, gestational weight gain; PA, physical activity; SCT Social Cognitive Theory; TTM, Trans Theoretical Model

\section{Midwives}

In designing the change strategies for midwives we again used the $\mathrm{TTM}^{271} \mathrm{SCT}^{272}$ and a third model: the Elaboration Likelihood Model (ELM). ${ }^{275}$ The first two theories are explained above. Using the insights of TTM, we sought to increase midwives' awareness of the problem of unhealthy GWG. Discussion, arguments, active learning, and cooperative learning, methods derived from the SCT, were used to increase midwives' knowledge, to influence their attitudes, correct misconceptions, and to create a virtual client of the type that midwives were supposed to support during pregnancy. Information about others' approval (SCT) was included to change midwives' perceptions of the social norms of their colleagues. Finally, guided practice and planning coping responses (SCT) were used to help midwives become more familiar with the new program and to help them to anticipate pregnant women's objections to change.

The ELM suggests that people have two different ways of processing information: central and peripheral. Central processing occurs when a message is carefully considered and compared against other messages and beliefs. Peripheral processing occurs when a message is processed without thoughtful consideration and comparison. ${ }^{275}$ According to Bartholomew et al., ${ }^{62}$ processing is related to higher persistence of attitude change, higher resistance to counter persuasion, and a stronger consistency between attitude and behaviour. For this reason, central processing should be promoted as much as possible. Following the ELM recommendations, we derived three ways to stimulate a thoughtful approach to our message: make the message personally relevant, unexpected, and repeat it regularly. Like we did with pregnant women, the methods chosen were combined into practical strategies. First, during training sessions midwives were informed about issues related to adequate GWG, in order to raise awareness of the problem and of their role as midwives as guardians of adequate GWG. Furthermore, the training included discussions (about participant's experiences, facts, perceived barriers all related to GWG) and active learning (working with the tailoring program, composing an individualized and regional overview of 
available health professionals they might consult). To support midwives' face-to-face communication with pregnant women, an information sheet about healthy GWG and with suggestions about proper communication was developed.

Table 7.5. Methods and strategies per determinant for midwives

\begin{tabular}{|c|c|c|c|c|}
\hline Determinant & $\begin{array}{l}\text { Change } \\
\text { objectives }\end{array}$ & Method & Theory & Strategy \\
\hline Awareness & $\begin{array}{l}\text { aw1a, } \\
\text { aw2a, } \\
\text { aw3a, } \\
\text { aw5a, } \\
\text { aw5b, aw5c }\end{array}$ & $\begin{array}{l}\text { Conscious- } \\
\text { ness raising }\end{array}$ & $\begin{array}{l}\mathrm{TTM}^{271} \\
\mathrm{SCT}^{272}\end{array}$ & $\begin{array}{l}\text { Presentation at start of training with information, } \\
\text { confrontation about prevalence, causes, and } \\
\text { consequences of unhealthy GWG and invitation for } \\
\text { feedback. } \\
\text { Attention for the easy access to junk food and for } \\
\text { healthy alternatives (like raw vegetables instead of } \\
\text { cookies). } \\
\text { Attending, gaining experience with the program during } \\
\text { the training session. }\end{array}$ \\
\hline Knowledge & $\begin{array}{l}k 2 a, k 2 b, \\
k 2 c, k 2 d, \\
k 2 e, k 2 f \\
k 2 g, k 2 h, \\
k 2 i, k 2 j, k 2 k\end{array}$ & $\begin{array}{l}\text { Active } \\
\text { learning } \\
\text { Cooperative } \\
\text { learning } \\
\text { Discussion }\end{array}$ & $\begin{array}{l}\mathrm{ELM}^{275} \\
\mathrm{SCT}^{272}\end{array}$ & $\begin{array}{l}\text { At the start of the training, the trainer identifies with } \\
\text { participants most eye-catching issues related to GWG } \\
\text { (health advantages, consequences too high/low GWG, } \\
\text { diet, PA, well-being, appropriate GWG, etc.) followed by } \\
\text { a discussion about difficult situations in participants' } \\
\text { midwifery practices. } \\
\text { Information card } \\
\text { Attending, gaining experience with the web-based } \\
\text { tailored program together with colleagues during the } \\
\text { training session. }\end{array}$ \\
\hline Attitudes & $\begin{array}{l}\text { a1a, a1b, } \\
a 1 c, a 1 d, \\
a 2 a, a 3 a, \\
a 3 b, a 3 c, \\
a 4 a, \\
a 4 b, a 4 c \\
a 5 a, a 5 b, \\
a 5 c, a 5 d, \\
a 5 e, a 6 a\end{array}$ & $\begin{array}{l}\text { Arguments } \\
\text { Active } \\
\text { learning }\end{array}$ & $\begin{array}{l}\mathrm{ELM}^{275} \\
\mathrm{SCT}^{272}\end{array}$ & $\begin{array}{l}\text { Presentation at start of training with information, } \\
\text { confrontation about the discrepancy of what midwives } \\
\text { think pregnant women need and women's actual needs } \\
\text { (at the start of pregnancy). } \\
\text { Group discussion during training session about } \\
\text { important learning questions related to GWG, disclosing } \\
\text { the existing pros and cons. Discussion results in a } \\
\text { collective casus that will later in the training be used as } \\
\text { an exercise example. } \\
\text { Attending, gaining experience with the program (e.g., } \\
\text { with the GWG curve) together with colleagues during } \\
\text { the training session. } \\
\text { During training a group discussion to prime and } \\
\text { underpin the significance of the update of midwives' } \\
\text { social network (dietician, physiotherapist, coach); who } \\
\text { can help them, how is the actual/ ideal cooperation, } \\
\text { what can help to stimulate an effective cooperation }\end{array}$ \\
\hline $\begin{array}{l}\text { Social } \\
\text { influences }\end{array}$ & $\begin{array}{l}\text { si2a, si3a, } \\
\text { si5a, si5b, } \\
\text { si5c, si5d, }\end{array}$ & $\begin{array}{l}\text { Information } \\
\text { about others' } \\
\text { approval }\end{array}$ & $\mathrm{SCT}^{272}$ & $\begin{array}{l}\text { Presentation (oral and visual) during training with } \\
\text { information about pregnant women's needs for } \\
\text { information about GWG in particular and lifestyle in } \\
\text { general, early in pregnancy. } \\
\text { Attending, gaining experience with the program } \\
\text { together with colleagues during the training session. }\end{array}$ \\
\hline
\end{tabular}


Discussion with midwives: During training a group discussion to prime and underpin the significance of the update of midwives' social network (dietician, physiotherapist, coach); who can help them, how is the actual/ideal cooperation, what can help to stimulate an effective cooperation.

\begin{tabular}{|c|c|c|c|c|}
\hline $\begin{array}{l}\text { Self-efficacy/ } \\
\text { skills }\end{array}$ & $\begin{array}{l}\text { se2a, se2b, } \\
\text { se2c, se3a, } \\
\text { se3b, se4a, } \\
\text { se4b, se5a, } \\
\text { se5b, se5c, } \\
\text { se5d, se5e, } \\
\text { se7a, se8a }\end{array}$ & $\begin{array}{l}\text { Guided } \\
\text { practice } \\
\text { training } \\
\text { Planning } \\
\text { coping } \\
\text { responses }\end{array}$ & $\mathrm{SCT}^{272}$ & $\begin{array}{l}\text { During the training: } \\
\text { Instructions about/ exercise with/ feedback on the use } \\
\text { of the information card with special attention to } \\
\text { appropriate interview techniques } \\
\text { Instructions about calculation of adequate GWG } \\
\text { categories and practicing with information card } \\
\text { according to pre-pregnancy BMI group } \\
\text { Instructions for appropriate anticipating on specific } \\
\text { barriers of pregnant women. } \\
\text { Instructions about communication with other } \\
\text { professionals about appropriate referrals in case of } \\
\text { unhealthy GWG. } \\
\text { Attending, gaining experience with the program (e.g., } \\
\text { with shaping the GWG curve) together with colleagues } \\
\text { during the training session. }\end{array}$ \\
\hline Barriers & $\begin{array}{l}\text { b2b, b5b, } \\
\text { b6a, b7a }\end{array}$ & $\begin{array}{l}\text { Facilitating } \\
\text { Enactive } \\
\text { mastery }\end{array}$ & $\mathrm{SCT}^{272}$ & $\begin{array}{l}\text { Information card for midwives and web-based tailored } \\
\text { program for pregnant women to structure GWG } \\
\text { education and to make more efficiently use of expensive } \\
\text { time. } \\
\text { Guidelines for weight anamneses, for referral to } \\
\text { relevant health care professionals. } \\
\text { During training: start with/ adapt social card with } \\
\text { eligible local health care professionals (dieticians/ } \\
\text { exercise programs/ coach) } \\
\text { Explain during training that making use of the web- } \\
\text { based tailoring program saves time of the midwife. } \\
\text { Prepare midwives that further in pregnancy they need } \\
\text { to invest some time to pay extra attention to GWG with } \\
\text { their clients. }\end{array}$ \\
\hline
\end{tabular}

Abbreviations BMI, Body Mass Index; SCT, Social cognitive Theory; ELM, Elaboration Likelihood Model, GWG, gestational weight gain; PA, physical activity; TTM, Trans Theoretical Model

\subsubsection{Step IV Development of the intervention program}

Our intervention - targeting both pregnant women and community midwives- was titled "Come On!", a play on words (in Dutch) that refers to both GWG and to the need to do something.

\section{Intervention component for pregnant women}

For pregnant women we developed an online GWG-program, with the aim of informing pregnant women, in an early stage of pregnancy, about healthy GWG, healthy diet and PA, and providing ways to accomplish a healthy GWG. The first part of the program consisted of a web-based, tailored program, the completion of which took about 45 minutes. We tailored the program based on pre-pregnancy BMI-group (underweight, normal, overweight, obese), 
their TTM stages of change regarding achieving a healthy GWG, healthy diet and PA, and their need for information. Participants answered questions on weight, height, struggles with their bodyweight, knowledge of benefits and harms of too low or too high GWG. Information and feedback was provided based on participants' answers. Barriers to healthy diet were discussed when women indicated that those barriers existed. Depending on women's prepregnancy BMI and on her self-expressed weight gain goal, an ideal goal was suggested. In addition, the program included thirteen informational films on healthy GWG, pregnancy related and healthy diet (i.e., advice on listeriosis, toxoplasmosis, intake of fish, fruits and vegetables) with an actor "Peggy", in the role of health professional. Depending on answers given to questions about pre-pregnancy activities, a film on PA was shown. Inactive women were advised to become active and active women were encouraged to continue their active lifestyle. Active women performing a "high risk" sport (e.g., diving, football) were advised to switch to an alternative "low risk" sport (e.g., swimming, biking).

The second part of the program consisted of a monitoring tool. Women were invited to note their weight in the program regularly. An invitation was sent weekly during the first ten weeks, biweekly in weeks 11 to 19 , every third week in weeks 22 to 28 , and at a gestational age of 32,36 and 40 weeks. If a woman did not respond to an invitation, she was not invited again.

Figure 7.1 provides an example of an individualized graphical GWG growth curve adjusted to women's pre-pregnancy BMI, indicating whether GWG was between (middle / green area), above (upper / pink area) or below the IOM guidelines (under / blue area). At each interval, participants received feedback plus the advice to discuss the feedback with their midwife. Each following visit to the program "Come On!", the woman was invited to watch one or more of the ten short films showing pregnant women dealing with recognizable situations. These films aimed to increase women's awareness of healthy GWG and related behaviours (i.e., diet and PA), to demonstrate positive social support (e.g., from peers), to increase selfefficacy expectations (e.g., towards reaching healthy diet and healthy PA), and to suggest ways to overcome barriers (e.g., how to cope with cravings). Each short film lasted about three minutes. Films from the first session with "Peggy" could be reviewed as well. 


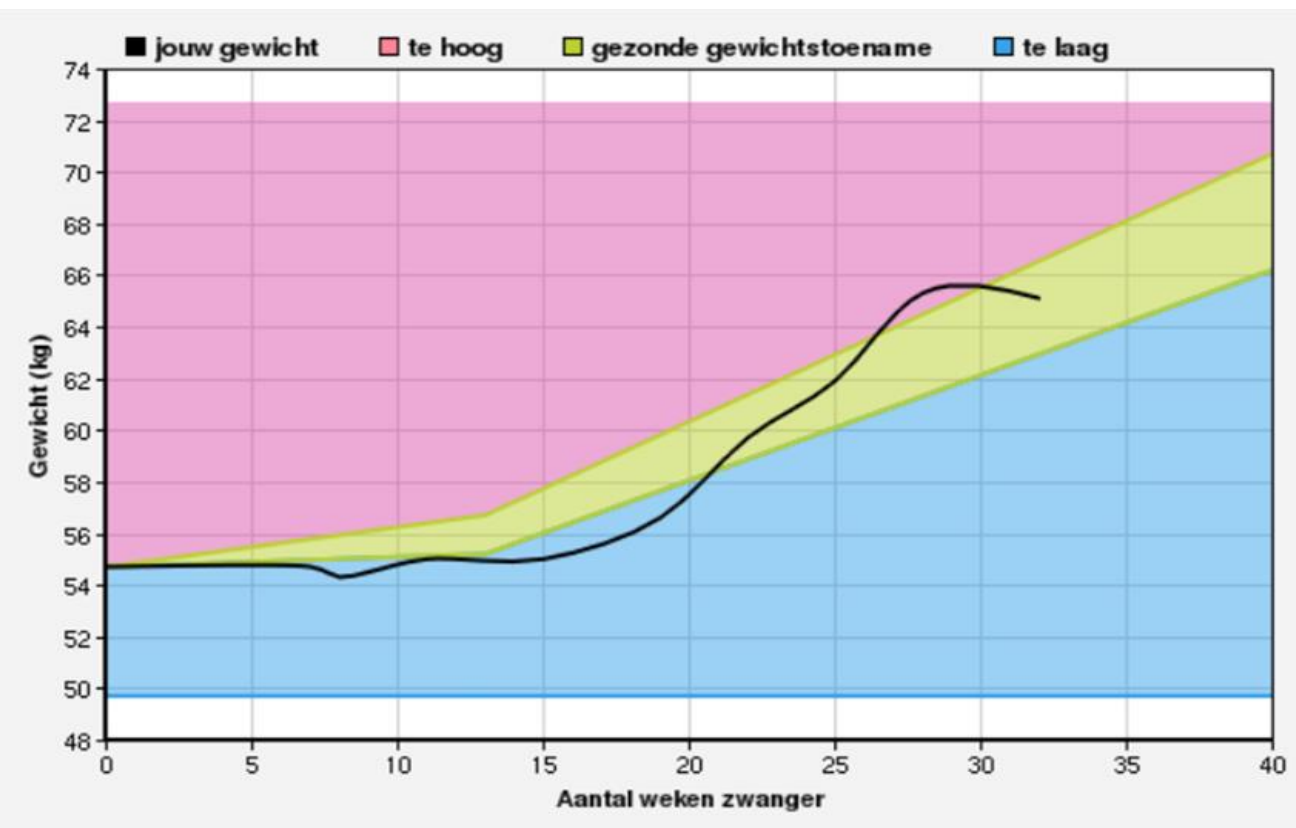

Figure 7.1 Graphical presentation of a pregnant woman's weight gain (an example) Notes, horizontal axis: \# weeks pregnancy; vertical axis: weight in kilogram; black line: actual weight; above area (pink, here dark grey): too high GWG; middle area (green, here light grey): adequate GWG; under area (blue, here dark grey): too low GWG

Abbreviation: GWG, Gestational weight gain

The online program was illuminated with a consistent logo and illustrations to increase attractiveness. Written language was kept at a level appropriate for women with low and moderate levels of education.

\section{Intervention component for midwives}

The intervention component for midwives included training and an information card. Because some midwives delegate certain activities (e.g., intake interview, lifestyle education) to practice assistants, these assistants were invited for the training as well. Midwives and assistants received four hours of training at a location in or nearby their working environment. The training was led by the first author (AM) who is experienced in professional education. In total, the training was delivered ten times; 60 midwives and twelve assistants participated. At the start of the training, participants were invited to talk about their experiences and perceived problems in relation to GWG with the aim of raising awareness of appropriate GWG and of their care provided to achieve appropriate GWG. They received oral and written information on the IOM-guidelines on GWG, the consequences of (un)healthy GWG, pregnant women's behaviours related to GWG, the determinants of those behaviours, and the behaviours of Dutch midwives concerning GWG and the determinants of those behaviours. The goal was to make midwives aware of the gap between pregnant women's 
needs and the current practice of midwives. The training introduced the online GWG program for pregnant women. Finally, midwives were advised to discuss GWG at least once during antenatal care, to discuss healthy diet and PA, and to refer women to a dietician and/or a PAprogram for pregnant women. Midwives were instructed about the use of the information card, which included summarised information, arguments for referral to a dietician, psychologist, PA-program and suggestions for questions. During the training, midwives created a list of regional healthcare workers they might refer their clients to or ask for advice. They were also encouraged to take a look at their own websites, in order to identify gaps in information about healthy lifestyles.

\subsubsection{Step V Development of an adoption and implementation plan}

Because the "Come On!" intervention is a newly developed program, we followed the recommendation of Bartholomew et al. to include step $V$ in the overall program planning ${ }^{62}$. Consortium midwives functioned as intermediaries between developers and the final users (pregnant women and midwives) during steps I-IV. Building on a relationship of trust with their pregnant clients, midwives collect broad understanding of their clients. The Consortium midwives and dietician provided valuable information about relevant impeding and enhancing factors concerning implementation in midwifery practices. For instance, to minimize midwives' time spent discussing healthy GWG, pregnant women can use the online "Come On!" program with minimal interference of the midwife, except for one single referral to the website. Midwives in the Consortium consulted with their colleagues in the field about the relevant (important and changeable) determinants that formed the starting point for "Come On!", and about selected practical strategies that were included. An essential part of "Come On!" was the training for midwives, organised to inform them about the purpose and procedures of the intervention and to practice skills that were part of the intervention, and at the same time to facilitate adoption and implementation. The pilot test (step VI) of the intervention produced information from pregnant women and midwives about their experiences and appreciation of the program, both of which were relevant for implementation.

\subsubsection{Step VI Development of an evaluation plan}

The effect of "Come On!" was evaluated in a non-randomized pre-post intervention study among pregnant women, comparing a historic control cohort (running May - August 2013) with an intervention cohort (running - April - July 2014). First, Consortium midwives recruited 17 midwives. Then, the participating midwives were entrusted with the task of including pregnant women for the control cohort and the intervention cohort. Midwives or their practice assistants informed adult pregnant women with a singleton pregnancy about the study during the first telephone contact. If women were open to information about the study, women's name and email address were collected and sent to the researcher (AM). The researcher then emailed the woman detailed information about the aim and procedures of the study. When the woman agreed to participate, she was invited to send a confirmation 
mail to the researcher. Following this email, the researcher sent a link to the first digital questionnaire. Before their first antenatal visit to the midwife, and at about 36 weeks of pregnancy, pregnant women completed an online questionnaire that was similar to the questionnaire used in the NA. Except for items on demographics in the first questionnaire (e.g., age, education), both questionnaires consisted of items on weight, diet, and PA. For process evaluation purposes, ${ }^{252}$ pregnant women from the intervention cohort answered questions related to exposure, use and appreciation of "Come On!" in the second questionnaire. Likewise, the midwives answered questions related to the quality of the training, fidelity and dose of the program delivered, usefulness of the "Come On!" program and barriers for use.

\subsection{Discussion and conclusion}

This paper presents a detailed outline of the process we used to develop "Come On!", a program intended to promote healthy GWG among healthy pregnant women. The NA (IM step I) provided the information about personal and environmental factors related to GWG that were needed to formulate program goals and specific change objectives. The use of a Consortium of advisors, starting with the first step of IM, ensured that we took into account individual and environmental factors related to GWG, and that we continuously incorporated the adoption and implementation preferences of the intended users. The goal of the "Come On!" program was to make healthy pregnant women aware of an adequate GWG and to provide them with tools to help them to stay within the IOM-guidelines for healthy GWG. The web-based part could be used at any time, at any place, was widely accessible in a relatively inexpensive way, and allowed for tailoring, all of which are favourable for effective health promotion efforts. ${ }^{276,277}$ The training for midwives fit with the current movement of lifelong learning. $^{278}$

One of the challenges during the development process was to translate results of existing studies focusing on specific target groups (e.g., overweight, obese, diabetes) to our population of healthy pregnant women. We dealt with this through our quantitative study of healthy pregnant women from the practices of community midwives ${ }^{188}$ and through the continuous interaction between the developers and the Consortium (midwives, dietician) who provided insights in GWG related behaviours, specific needs and applicability of selected strategies within our target groups. This interaction provided the empirical and theoretical foundation for the decision making process.

Although we found IM to be useful, it was also time-consuming. IM is typically applied to simple and uni-dimensional behaviours and can become unwieldy when applied to complex behaviours such as healthy GWG promotion. ${ }^{279}$ During the NA the complexity of GWG became clear, and use of IM resulted in the collection of a large and complex amount of information. Prioritizing based on impact and changeability resulted in the elimination of some relevant factors in favour of others judged to be more changeable and with a higher impact. The 
results of the effect and process evaluation studies will provide insights into the wisdom of these judgements.

We used a purposive sample of midwives and thus it is plausible that these midwives had above average interest in issues regarding GWG and were highly motivated to find solutions. This may distort a realistic view of the factors and solutions related to the problem of healthy GWG. However, according to Roger's Diffusion of Innovation theory, the involvement of early adopters - an apt description of the Consortium midwives - is necessary for the development and implementation of new initiatives. ${ }^{62,280}$ Also the recruitment of participants for the effect study by the Consortium midwives might have resulted in a study population that is not generalizable to the larger population of healthy pregnant women.

The "Come on!" intervention was developed in a careful and comprehensive way, which calls for an evaluation of the program in a wider, representative sample of pregnant women. We present the results of this evaluation study in chapter 8 . 


\section{Chapter 8}

\section{"Come on!" Cohort-study of an intervention, targeting on healthy gestational weight gain}

Astrid Merkx, Marlein Ausems, Luc Budé, Raymond de Vries, Marianne J. Nieuwenhuijze

Submitted 


\section{Background}

Approximately $40 \%$ of women gain more weight in pregnancy than recommended by the Institute of Medicine (IOM), which is a cause of concern, because excessive gestational weight gain (GWG) is a significant contributor to obesity for the mother and baby. We developed an evidence informed intervention: "Come On!". In this article we assess the effectiveness of our intervention.

\section{Methods}

We performed a non-randomised pre-post intervention study to explore the effectiveness of "Come On!". The intervention targeted both pregnant women and midwives. We compared a historic cohort (controls, $n=144$ ) with an intervention cohort $(n=129)$ of clients from midwifery practices in the Netherlands. We used inferential statistics to determine the effect of the intervention on GWG, diet and physical activity.

\section{Results}

While the intervention had no significant effects on GWG, diet, or physical activity, we found other factors to be associated with variations in GWG. Having an adequate weight gain goal promoted healthy GWG; meeting the fish-norm and higher education was positively associated with GWG within IOM-guidelines compared to above IOM-guidelines; full-time working and not meeting the fruit-norm was positively associated with GWG within the IOMguidelines compared to below IOM-guidelines. Women who stopped smoking gained more often GWG above IOM-guidelines.

\section{Conclusion}

Although the "Come on!" intervention was not effective in promoting GWG within the IOMguidelines, our implementation and evaluation provide a better understanding of what is needed to have a healthy GWG. Our results can be used to inform future interventions targeting GWG. 


\subsection{Introduction}

To improve the health of mother and babies, it is critical to encourage women to have appropriate gestational weight gain (GWG). Too much or too little GWG is associated with negative obstetric outcomes including gestational diabetes, higher caesarean section rates and prematurity. ${ }^{18,22,47,122}$ Furthermore, high GWG is associated with overweight and obesity in the long term for both mothers and their babies. ${ }^{6}$ The Institute of Medicine (IOM) has published guidelines for healthy GWG, with cut-off points related to pre pregnancy Body Mass Index (BMI). ${ }^{6}$ The IOM-guidelines recommend in total 0.5 to $2.0 \mathrm{~kg} \mathrm{GWG}$ for the first trimester and depending on the BMI 5-9 kg for obese to 12.5-18.0 kg for underweight women. ${ }^{6}$ In order to promote the health of mothers and babies in the Netherlands -where only one-third of healthy pregnant women have a GWG within the IOM-guidelines- $-{ }^{45,188}$ we searched for an affordable and effective intervention that targeted healthy pregnant women and their prenatal care providers. Our search was unsuccessful, but in reviewing the literature, we found information that we could use to develop our own intervention. We discovered for example that existing interventions differ in effectiveness, are limited to specific risk groups (such as obese women or women with gestational diabetes mellitus), or use only one approach (e.g., behavioural counselling, diet, or physical activity (PA)). ${ }^{145,216}$ With regard to the role of caregivers in the management of GWG, we learned that knowledge, beliefs about consequences, and environmental context are critical determinants of the implementation of programs for preventing too much $\mathrm{GWG}^{281}$ In particular, a Dutch study showed that midwives consider discussions of GWG with clients to be time consuming, an attitude that impedes the implementation of GWG guidelines. ${ }^{204}$

Given the lack of effective programs, it was necessary to develop an intervention and we chose to target healthy pregnant women and their midwives. To build the intervention, we followed the Intervention Mapping (IM) protocol, ${ }^{62}$ a process that includes a thorough needs assessment, goal specification, and the use of relevant theories and applications in building the program. In order to ensure that our intervention fit with the practical experiences of clinicians we organized a Consortium, consisting of three practicing midwives, a dietician, a physiotherapist and experts on health promotion. We named our intervention "Come On!" a play on words in Dutch, which can mean "do it!" and "put on weight". Further details of the development of "Come on!" are described elsewhere. ${ }^{282}$ In this paper, we describe the effects of our intervention on GWG, diet and PA.

The Research Ethics Committee of Atrium-Orbis-Zuyd reviewed the study protocol and provided ethical approval (number 14-N-41). The trial study was registered in the Netherlands National Trial Register (NTR) under number NTR4717. 


\subsection{Methods}

To evaluate the effectiveness of the "Come On!" intervention we designed a non-randomised pre-post-test with a historical cohort as control group.

\subsubsection{Procedures and participants}

The three Consortium midwives recruited seventeen midwifery practices to participate in the study. The midwives/assistants from these practices recruited pregnant women for the control group between May and August 2013 and for the intervention group between April and July 2014. The recruiters were asked to tell pregnant women, aged 18 years or older, about the study during the first contact (by telephone) and to determine if the woman was open to receiving detailed information about the study. If a woman agreed, the recruiter collected her name and email address and sent these to the researcher (AM), who then emailed the woman detailed information about the aim and procedures of the study. If a woman decided to participate, she sent a confirmation email to the researcher. Following this confirmation, the researcher sent a link to the pre-test digital questionnaire (Q1). Women in the intervention group received a link to the online GWG-program after completing the pretest questionnaire. At a gestational age of 36 weeks, the midwifery practice was contacted to determine if the woman was still pregnant. Women with an intact pregnancy were sent the link to the post-test questionnaire (Q2). If the questionnaire was not completed, reminders were sent after four and eight days.

Although we did not have enough prior information to calculate an adequate sample size, we determined that we would need to collect at least 200 participants in each group in order to be able to detect a medium effect.

\subsection{2 "Come on!" intervention}

The development of "Come On!" was finished in March 2014. Our intervention targeted healthy pregnant women and their midwives/assistants.

\section{Pregnant women}

The intervention for pregnant women had two main components: (1) A web-based tailored program that started in very early pregnancy (as soon as the woman was enrolled in prenatal care) and continued until the end of pregnancy; and (2) regular face-to-face discussions between the pregnant woman and their midwife about GWG, diet, and PA during the first half of pregnancy.

The online GWG-program was split into a major first session of 45 minutes, general and tailored messages about GWG, diet and PA, short follow-up sessions (maximum thirteen) and a personalised GWG graph for monitoring GWG. The major session included nine movie clips with "Peggy", a woman who was presented as an expert in the field of pregnancy and GWG, as well as illustrations and easy to read texts. Women were invited by email to use the followup sessions. In these sessions, the personalised GWG-graph was displayed and the woman 
was given the option to watch one of the "Peggy" clips or one of the ten short films in which a peer demonstrated her challenge to achieve a healthy GWG.

\section{Midwives/assistants}

For midwives/assistants we developed a 4-hour training, which was coordinated by an expert in professional development and took place in April 2014 at a location chosen by the participants. Participants reflected on their experiences with GWG, diet, and PA and related problems. Subsequently, they received information about the IOM-guidelines, the consequences of healthy and unhealthy GWG, and a summary of the behaviours of pregnant women and Dutch midwives regarding GWG. Participants also gained experience with the online program "Come On!". They were trained to discuss GWG with women at least once during prenatal care. Participants received an information card with a summary of GWG information. Finally, they were invited to critically look at the consumer information on their websites and adjust it if necessary. The trainer encouraged midwives to contact local colleagues for help, such as a dietician, an expert in PA, and/or a psychologist.

\subsubsection{Questionnaire}

Demographic information was solicited only on the first questionnaires. Both questionnaires (Q1 at the start of pregnancy (T1) and Q2 at 36 weeks (T2)) consisted of items on height, weight, diet, and PA. We asked for pre-pregnancy weight, and weight (and date of weighing) at the end of pregnancy. Diet was measured with: six items from the Food Frequency Questionnaire (FFQ) for vegetables (\#days per week, boiled and/or raw vegetables, \#gram per day), ${ }^{283}$ two items about the consumption of fish (\#days per week, consumption of fatty fish), and two items about the consumption of fruit (\#days per week, \#pieces per day). When answering items from the FFQ-vegetables, respondents chose between photographs of plates with different amounts of vegetables. ${ }^{149,188}$ PA was measured with items on the number of minutes the participant engaged in walking, cycling, sports, short rigorous activities, and working in the home and garden at each day of the week. ${ }^{188}$ During the intervention period we encouraged participants to email the program developers with feedback and collected (and answered) these emails.

\section{Primary and secondary outcomes}

As primary outcome we used GWG in relation to the IOM-guidelines (below, within, or above recommended GWG), using the self-reported pre-pregnancy BMI. Because the IOMguidelines relate GWG to gestational age, we calculated GWG in relation to the gestational age; we used the date of last weighing for this measurement. Secondary outcomes were the vegetable consumption at $\mathrm{T} 2$, the percentage of women meeting the fruit-norm and the fishnorm at T2, and PA at T2. Because PA at T1 and T2 were not normally distributed, we used the difference of $P A$ between $\mathrm{T} 2$ and $\mathrm{T} 1$-which was distributed normally- as a secondary outcome. 


\section{Confounders}

As confounders we identified pre-pregnancy BMI, ethnicity (Dutch if both parents were born in the Netherlands, otherwise non-Dutch), age (in years, at T1), parity (nulliparous or parous), family-income (low when under median Dutch household income (33,000 euro/year), otherwise high), education (low: without high school diploma; middle: with high school diploma; high: with university education), smoking (non-smoking, stopped, continued), GWGgoal (below, within or above IOM-recommendations), work (full-time, part time, no paid job), sleep (mean of \# hours last week) and satisfaction with pre-pregnancy weight (7-point Likert scale; $1=$ very dissatisfied, $7=$ =very satisfied). We computed dummy variables for the categorical variables with more than two levels (education, smoking, GWG-goal and work), with medium education, non-smoking, GWG-goal within IOM-guidelines, and part-time work as reference categories.

\subsubsection{Data-analyses}

We first checked our data for normality. To insure that results were not affected by drop-out, we performed a logistic regression analyses with drop-out at $\mathrm{T} 2$ as dependent variable ( $1=$ drop-out; $0=$ data completed) and included study group, vegetable consumption, fruitnorm, fish-norm at T1 as independent variables and corrected for confounders. Differences between the control and intervention group at T1 were tested with student T-tests for continuous variables and Chi square tests for categorical variables. We corrected for multiple testing with Bonferroni. ${ }^{284}$

To test the effectiveness of the intervention, we performed a multinomial regression analysis with GWG in relation to the IOM-guidelines as dependent variable (categories below - within above IOM-guidelines), contrasting (1) participants gaining below with participants gaining within the IOM-guidelines, and (2) participants gaining within with participants gaining above the IOM-guidelines) and the study group (control/intervention) as an independent variable. We controlled for consumption of vegetables, Fish norm, Fruit norm, and above-mentioned confounders, all measured at T1. The effect of the intervention on secondary outcomes was tested with linear regression (dependent variables = consumption of vegetables at $\mathrm{T} 2$ and difference in PA) and logistic regression (dependent variables = fruit norm and fish norm at T2).

\subsection{Results}

\subsubsection{Participants}

We recruited 230 women for the control group and 238 women for the intervention group. In the end we analysed data from 273 participants - 144 women in the control and 129 women in the intervention group (Figure 8.1). 


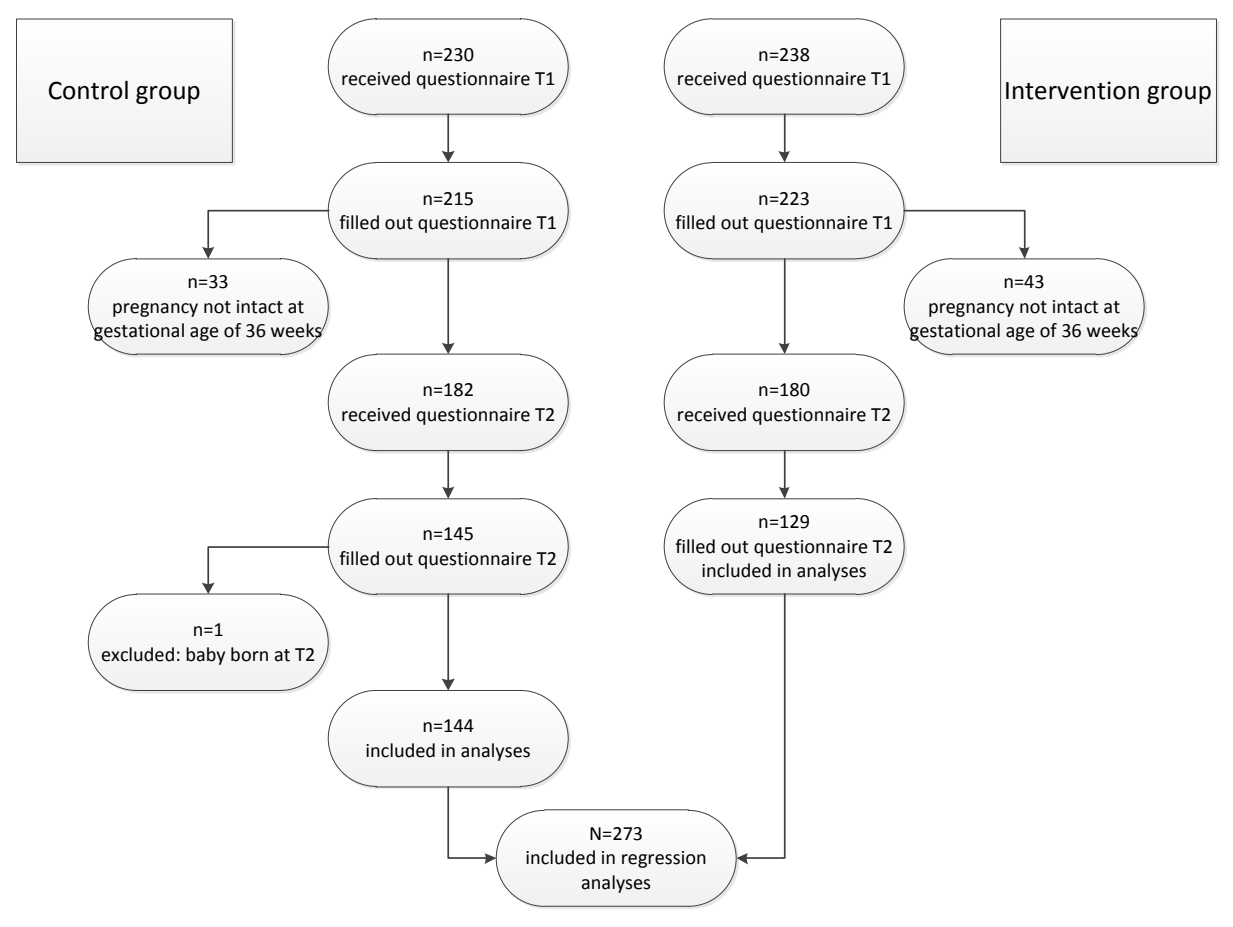

Figure 8.1 Flow chart of participants included in analyses

The drop-out analyses showed no differences between drop out at $\mathrm{T} 2$ in the control and intervention groups, with one exception: women meeting the Fish-norm were more likely to drop out (OR 1.84, 95\% Cl 1.05-3.23).

Table 8.1 shows pre-test characteristics. We found no statistically significant differences between the control and intervention group (note that because of Bonferroni correction, level of significance is reached with $p<0.003$ ).

Table 8.1 Baseline characteristics control group $(n=215)$ and intervention group $(n=223)$

$$
\text { Control group Intervention group }
$$

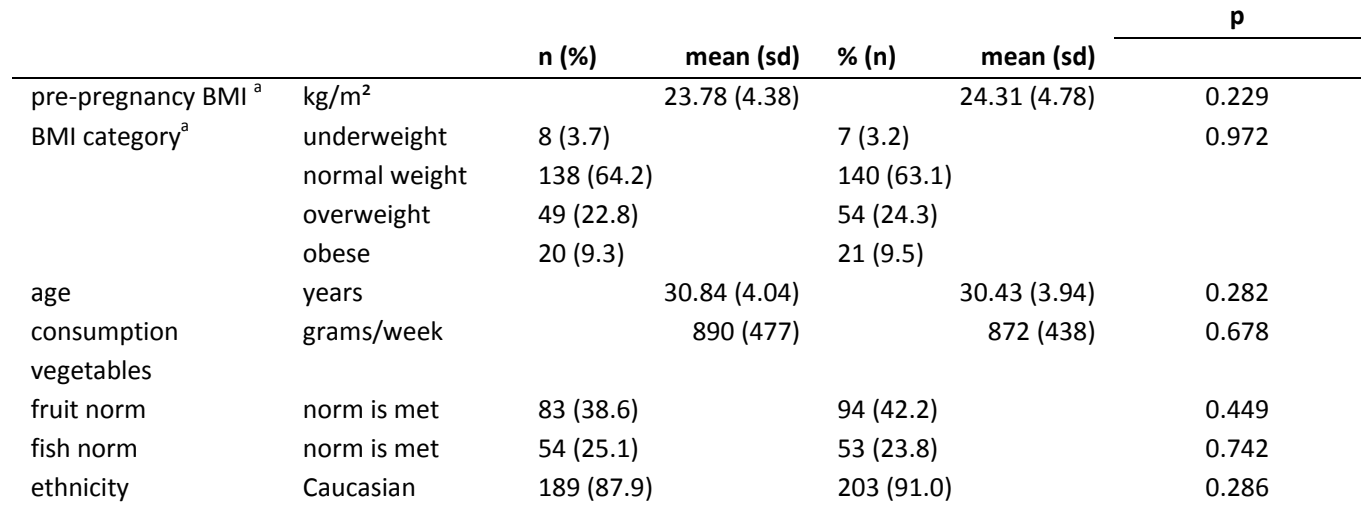




\begin{tabular}{|c|c|c|c|c|c|}
\hline parity & nulliparous & $103(47.9)$ & & 118 (52.9) & 0.295 \\
\hline family income & above modal & $147(68.4)$ & & $152(68.2)$ & 0.962 \\
\hline \multirow[t]{3}{*}{ education } & low & $11(5.1)$ & & $24(10.8)$ & 0.042 \\
\hline & middle & $80(37.2)$ & & 66 (29.6) & \\
\hline & high & $124(57.7)$ & & $133(59.6)$ & \\
\hline \multirow[t]{3}{*}{ smoking } & stopped & $33(15.3)$ & & $17(7.6)$ & 0.027 \\
\hline & non-smoking & $168(78.1)$ & & $185(83.0)$ & \\
\hline & continued & $14(6.5)$ & & $21(9.4)$ & \\
\hline \multirow[t]{3}{*}{ GWG goal ${ }^{b, c}$} & below IOM & $52(28.1)$ & & $59(31.2)$ & 0.583 \\
\hline & within IOM & 99 (53.5) & & 91 (48.1) & \\
\hline & under IOM & $34(18.4)$ & & $39(20.6)$ & \\
\hline \multirow[t]{3}{*}{ work } & full-time & $97(45.1)$ & & $103(46.2)$ & 0.167 \\
\hline & part-time & $100(46.5)$ & & $93(41.7)$ & \\
\hline & no paid job & $18(8.4)$ & & 27 (12.1) & \\
\hline sleep ${ }^{d, e}$ & hours/24 hour & & $8.13(1.30)$ & $8.15(1.42)$ & 0.893 \\
\hline $\begin{array}{l}\text { satisfied with weight } \\
f\end{array}$ & $\begin{array}{l}\text { 1=very } \\
\text { dissatisfied, } \\
\text { 7=very satisfied }\end{array}$ & & $4.82(1.67)$ & $4.87(1.65)$ & 0.790 \\
\hline
\end{tabular}

\subsubsection{Primary outcome}

Table 8.2 shows the results of the multinomial regression analysis, comparing respondents gaining weight below versus within the IOM-guidelines, and comparing respondents gaining above versus within. We found no effect of the intervention on GWG. We did, however, find a few associations between our dependent and independent variables. Comparing GWG below and within the guidelines, women who met the fruit-norm and women who worked full-time were less likely to gain weight below the guidelines, women who had a too low GWG-goal were more likely to gain weight below the guidelines. Comparing GWG within and above the guidelines, women who stopped smoking and women whose goal for GWG was above the IOM-guidelines were more likely to have a GWG above the guidelines; women who met the fish-norm and women who were highly educated were less likely to gain weight above the guidelines. Pre-pregnancy BMI was not significantly correlated with GWG within the guidelines.

Table 8.2 Results multinomial regression analysis with gaining weight within guidelines of Institute of Medicine (IOM) as reference ('below' $n=28$; 'within' $n=117$, 'above' $n=128$ )

\begin{tabular}{lcccc} 
& \multicolumn{2}{c}{ below versus within IOM } & \multicolumn{2}{c}{ above versus within IOM } \\
\cline { 2 - 5 } & $\mathrm{p}$ & $\mathrm{OR}(95 \% \mathrm{Cl})$ & $\mathrm{p}$ & $\mathrm{OR}(95 \% \mathrm{Cl})$ \\
\hline study group (0=control; 1=intervention) & 0.698 & $1.21(0.46-3.24)$ & 0.536 & $0.83(0.46-1.50)$ \\
\hline pre-pregnancy BMI $\left(\mathrm{kg} / \mathrm{m}^{2}\right)$ & 0.058 & $1.13(1.00-1.28)$ & 0.676 & $1.02(0.93-1.11)$ \\
\hline vegetable consumption (grams/week) & 0.560 & $1.04(0.92-1.17)$ & 0.164 & $1.05(0.98-1.13)$ \\
\hline fish-norm (0=not met; 1=met) & 0.397 & $1.63(0.53-5.06)$ & $\mathbf{0 . 0 0 5}$ & $\mathbf{2 . 8 2}(\mathbf{1 . 3 6 - 5 . 8 6 )}$ \\
\hline fruit-norm (0=not met; 1=met) & $\mathbf{0 . 0 3 0}$ & $\mathbf{3 . 1 3 ( 1 . 1 2 - 8 . 7 6 )}$ & 0.079 & $1.72(0.94-3.14)$ \\
\hline ethnicity (0=Dutch; 1=non-Dutch) & 0.279 & $0.29(0.03-2.70)$ & 0.591 & $0.77(0.29-2.02)$ \\
\hline
\end{tabular}




\begin{tabular}{|c|c|c|c|c|c|c|}
\hline \multicolumn{2}{|l|}{ age (years) } & 0.597 & $0.96(0.83-1.11)$ & 0.331 & \multicolumn{2}{|c|}{$1.04(0.96-1.14)$} \\
\hline \multicolumn{2}{|c|}{ parity ( $0=$ nulliparous; $1=$ parous) } & 0.399 & $1.57(0.55-4.47)$ & 0.173 & \multicolumn{2}{|c|}{$1.59(0.82-3.09)$} \\
\hline \multicolumn{2}{|c|}{$\begin{array}{l}\text { family income ( } 0=\text { below; } 1=\text { above } \\
\text { modal) }\end{array}$} & 0.411 & $0.60(0.17-2.05)$ & 0.661 & \multicolumn{2}{|c|}{$1.17(0.58-2.39)$} \\
\hline \multirow[t]{3}{*}{ education } & low $(=1)$ & 0.339 & $0.42(0.07-2.48)$ & 0.184 & \multicolumn{2}{|c|}{$2.32(0.67-8.05)$} \\
\hline & high (=1) & 0.537 & $1.47(0.44-4.91)$ & 0.005 & \multicolumn{2}{|c|}{$2.87(1.39-5.95)$} \\
\hline & middle & & reference & & \multicolumn{2}{|c|}{ reference } \\
\hline \multirow[t]{3}{*}{ smoking } & stopped (=1) & 0.462 & $0.51(0.08-3.10)$ & 0.035 & \multicolumn{2}{|c|}{$0.32(0.11-0.92)$} \\
\hline & continued (=1) & 0.800 & $0.77(0.10-6.03)$ & 0.212 & \multicolumn{2}{|c|}{$0.41(0.10-1.67)$} \\
\hline & non-smoking & & reference & & \multicolumn{2}{|c|}{ reference } \\
\hline \multirow[t]{3}{*}{ GWG-goal } & below IOM (=1) & 0.010 & $0.23(0.07-0.70)$ & 0.969 & \multicolumn{2}{|c|}{$1.01(0.49-2.10)$} \\
\hline & above IOM (=1) & 0.812 & $0.85(0.21-3.38)$ & 0.029 & \multicolumn{2}{|c|}{$0.40(0.17-.91)$} \\
\hline & within IOM & & reference & & \multicolumn{2}{|c|}{ reference } \\
\hline \multirow[t]{3}{*}{ work } & full-time (=1) & 0.013 & $4.46(1.38-14.45)$ & 0.387 & \multicolumn{2}{|c|}{$1.34(0.69-2.62)$} \\
\hline & no paid job (=1) & 0.873 & $0.88(0.19-4.07)$ & 0.915 & \multicolumn{2}{|c|}{$1.06(0.36-3.18)$} \\
\hline & part-time & & reference & & \multicolumn{2}{|c|}{ reference } \\
\hline \multicolumn{2}{|c|}{ sleep T1 (hours / 24 hours) } & 0.904 & $1.02(0.70-1.49)$ & 0.130 & \multicolumn{2}{|c|}{$1.22(0.94-1.59)$} \\
\hline \multicolumn{2}{|c|}{ satisfied with weight $T 1^{\mathrm{a}}$} & 0.371 & $0.85(0.59-1.22)$ & 0.566 & \multicolumn{2}{|c|}{$0.94(0.76-1.17)$} \\
\hline \multicolumn{7}{|c|}{$\begin{array}{l}\text { Note: } \mathrm{R}^{2}=.256 \text { (Cox and Snell), } .301 \text { (Nagelkerke) } \chi^{2} \\
\text { Abbreviations: BMI, Body Mass Index; Cl, Confiden } \\
\text { point } 1 \text { (first trimester) } \\
\text { a Likert scale; } 1=\text { very dissatisfied - } 7=\text { very satisfied }\end{array}$} \\
\hline \multicolumn{7}{|c|}{ 8.3.3 Secondary outcomes } \\
\hline \multicolumn{7}{|c|}{$\begin{array}{l}\text { The result was the same for our secondar } \\
\text { effect of the intervention (Table 8.3). Die } \\
\text { had lower vegetable consumption at T2. }\end{array}$} \\
\hline \multicolumn{7}{|c|}{ 8.3.4 Use of the program } \\
\hline \multicolumn{7}{|c|}{$\begin{array}{l}\text { During the intervention period, we received a number of emails from participants, mainly } \\
\text { about the consumer friendliness of the program. Participants found it difficult to fill out their } \\
\text { gestational age, the program required for instance choosing between } 11 \text { or } 13 \text { weeks of } \\
\text { pregnancy. Furthermore, some participants encountered problems with changing the due } \\
\text { date and some reported they were not invited to revisit the program anymore. }\end{array}$} \\
\hline \multicolumn{7}{|c|}{$\begin{array}{l}\text { Table } 8.3 \text { Results logistic and linear regression analyses with secondary outcomes, diet and } \\
\text { physical activity (PA) }\end{array}$} \\
\hline & & & $\begin{array}{l}\text { standardized } \\
\text { coefficients }\end{array}$ & $\begin{array}{r}\text { OR }(95 \% \text { co } \\
\text { inter }\end{array}$ & fidence & $\mathbf{p}$ \\
\hline & ssion analysis: $\mathrm{Fr}$ & norm $n$ & $\mathrm{~T}^{\mathrm{a}}{ }^{\mathrm{a}}$ ot met $(0) \mathrm{n}=$ & , met (1) $n=$ & & \\
\hline study gro & & & & $0.846(0.47$ & $-1.522)$ & 0.577 \\
\hline fruit norn & & & & $0.194(0.1 C$ & $-0.349)$ & 0.000 \\
\hline vegetable & ion T1 (grams/we & & & $1.001(1.0 c$ & $-1.002)$ & 0.000 \\
\hline
\end{tabular}


Logistic regression analysis: Fish-norm met $T 2^{b}$ not met (0) $n=224$, met (1) $n=49$

study group

$1.259(0.608-2.606)$

0.535

fish norm T1

$0.217(0.103-0.455)$

0.000

Linear regression analysis: Vegetable consumption (grams/week) T2 ${ }^{\mathrm{c}}$ mean consumption 873 (sd 401 )

$\begin{array}{lcr}\text { study group } & -0.022 & 0.641 \\ \text { vegetable consumption T1 } & 0.590 & 0.000 \\ \text { fruit norm T1 } & 0.136 & 0.005 \\ \text { parity } & -0.110 & 0.036\end{array}$

Linear regression analysis: Difference PA T2-T1, (minutes/week) ${ }^{d}$ mean difference PA T2-T1 -53 (sd 426)

study group

0.068

0.288

Abbreviations: BMI, Body Mass Index; OR, Odds ratio; PA, physical activity; T1, time point 1 (first trimester); T2, time point 2 (36 weeks gestation); sd, standard deviation

${ }^{\mathrm{a}} \mathrm{R}^{2}=.258$ (Cox and Snell), .344 (Nagelkerke) $\chi^{2}$ (df18)=81.352 p=0.000; also included, but not significant: smoking, education, goal, work, age, ethnicity, parity, pre-pregnancy BMI, sleep, satisfied with weight

${ }^{\mathrm{b}} \mathrm{R}^{2}=.138$ (Cox and Snell), .226 (Nagelkerke) $\chi^{2}$ (df18)=81.352 $\mathrm{p}=0.002$; also included, but not significant: vegetable consumption T1, fruit norm T1, smoking, education, goal, work, age, ethnicity, parity, pre-pregnancy BMI, sleep, satisfied with weight

${ }^{c} R^{2}=.487 p=0.000$; also included, but not significant: smoking, education, goal, work, age, ethnicity, pre-pregnancy $\mathrm{BMI}$, sleep, satisfied with weight

${ }^{d} R^{2}=.056 p=0.443$; also included but not significant: smoking, education, goal, work, age, ethnicity, parity, prepregnancy BMI, sleep, satisfied with weight

\subsection{Discussion}

The overall goal of our study was to find a way to increase the likelihood for healthy pregnant women to gain weight within the IOM-guidelines. We developed the intervention using an evidence informed protocol and characteristics of the intervention group and the control group were comparable. In spite of this, we observed no effect of the intervention on GWG, diet and PA.

In the 2015 update of the 2012 Cochrane review ${ }^{145}$ of diet and PA interventions to achieve a healthy GWG, Muktabhant et al., ${ }^{58}$ found that overall weight management interventions (for all target groups) led to a $20 \%$ reduction in the number of women gaining weight above the guidelines (RR of gaining weight above the guidelines $0.8095 \% \mathrm{Cl} 0.73-0.87 ; n=24$ studies). Dietary interventions and supervised PA interventions were the most promising interventions in this respect. However, for a low risk population, only a few studies showed a beneficial effect of an intervention. Earlier research from the Netherlands also discovered that it is difficult to successfully intervene to control GWG. ${ }^{285}$

An intervention study of GWG in Germany using two extra consultations (one of 60 minutes and one of 30 minutes per woman) showed positive results (OR 0.5, 95\% Cl 0.3-0.9 for excessive GWG compared to not excessive GWG). ${ }^{126}$ To prevent an unsustainable increase in time investment for midwives during prenatal care, the "Come On!" intervention used an online tailoring program. Studies of technology supported interventions seeking to influence lifestyle in pregnant women are scarce. ${ }^{286}$ In another area, however, (online interventions on 
mindfulness to improve mental health) these interventions were proved to be successful. ${ }^{287} \mathrm{~A}$ review of e-Health interventions on weight-management in non-pregnant adults showed promising results as well. ${ }^{288} \mathrm{~A}$ Dutch intervention, that also combined a tailored website with a training for midwives, was successful in reducing and preventing maternal distress. ${ }^{289}$ It is thinkable that e-Health interventions work well when they are user friendly and when the users feel that their own caregiver supports the intervention. In our situation, the intervention was supported by the women's own midwives, but, as it was newly developed, we discovered some unexpected "skeletons in the cupboard", reflecting the content of the emails that were sent by the participants during the study. This likely influenced its success. A more thorough process analysis of the intervention is planned and will shed more light on the reasons why the intervention did not have effect on GWG.

In our research, as in an earlier Dutch study, ${ }^{188}$ pre-pregnancy BMI was not associated with gaining weight outside the guidelines, although it is often found to be associated. ${ }^{50}$ Further research is needed to find out if healthy overweight and obese women have an additional risk factor for excessive GWG compared to high risk overweight and obese women. Our study confirmed earlier findings that women with an appropriate GWG goal, and highly educated women more often gain weight within the guidelines. ${ }^{50,188,215}$ Our study contradicted with the findings of an earlier study that found higher fish consumption to be correlated with a higher GWG, ${ }^{53}$ we do not understand this differences.

Working full-time (compared to part-time) appeared to be related with not gaining too low GWG. We did not find such relationship in literature and consider it as a type I error.

\subsubsection{Strengths and limitations}

The design of this study is not as powerful as a randomised controlled trial. However, collection of data was in two subsequent years, in the same period of the year, in the same midwifery practices and with similar populations, which increased its comparability. No major changes in policies or guidelines for GWG, nutrition or PA took place in that period.

Furthermore, with a historic cohort we avoided contamination between women receiving the intervention and women not receiving it.

To our knowledge, this is the first intervention aiming to promote healthy GWG developed with the IM protocol. Although the "Come on!" intervention was not yet effective in promoting healthy GWG, we think it contributes to a better understanding of what is needed to have a healthy GWG, which is useful information because the burden of overweight and obesity still exists.

\subsubsection{Conclusion}

Our intervention was not yet successful in increasing the amount of healthy women with a healthy GWG. In addition to this, we confirmed earlier findings that goal setting is important to achieve a healthy GWG and that pre-pregnancy BMI was not associated with GWG within the IOM-guidelines. 
Chapter 9

\section{Summary and general discussion}




\subsection{Introduction}

This thesis opened with the following general research question: Can we develop an evidence based intervention to support Dutch primary care midwives in the effective promotion of a healthy gestational weight gain (GWG) for women in their care? The answer to this general question is short: In the time available to us, we were unable to design and implement an intervention to do this (chapter 8). However, we were able to learn about the many aspects of every day midwifery practice that can contribute to answers relating to healthy GWG. Since both mothers and their babies have higher risks of negative outcomes when GWG is too high or too low, ${ }^{6,18-30,55,290}$ and because most women have GWG outside the frequently used Institute of Medicine (IOM)-guidelines, ${ }^{44,45,188}$ it is important to help midwives and women to find a balance in GWG; neither too much, nor too little.

Although our intervention "Come on!" was not successful, we were able to answer the several sub-questions generated by our general research question. These answers provided important information contributing to the developing process of "Come on!", and also to the general body of knowledge on GWG (chapters 2-7). In this general discussion we present the main findings (9.2), discuss these findings (9.3), consider the methodological limitations and strengths of our work (9.4), address the implications of our findings for maternity care practice (9.5), and offer suggestions for future research (9.6).

\subsection{Summary of main findings}

In our project we focussed on GWG, a topic relevant for women in their childbearing years, their offspring, health professionals in maternity care, and, more generally for public health policies (chapter 1). In our review on interventions targeting on GWG (chapter 2), we found that dietary and physical activity (PA) interventions for obese women are promising ways to approach the reduction of GWG, especially when diet is supported with coaching. No effective intervention was found for GWG in healthy women regardless of pre-pregnancy BMI-group (chapter 2).

In our cohort study of 455 healthy pregnant women of all gestational ages, receiving care from midwives in community practices (chapters 3 and 4 ) we found that $42 \%$ gained weight within, $14 \%$ below, and $44 \%$ above the IOM-guidelines (calculated for their gestational age). GWG within the guidelines, compared to GWG above and below the guidelines, was not associated with pre-pregnancy BMI or with diet. Women who reduced their PA were twice as likely to experience GWG above the guidelines. Non-Caucasian women were five times more likely, and women who stopped smoking were twice as likely, to have a GWG above the IOMguidelines. When compared with insufficient GWG, there was a significant association between healthy GWG and perceived higher sleep deprivation (an increase in reported sleep deprivation of one day per week gave a $20 \%$ greater chance of GWG within IOM-guidelines). Mean vegetable consumption was 949 grams per week. Less than half of pregnant women (46.4\%) met the fruit norm and only a small part of our sample (6.4\%) met the fish norm. 
Seventy-five percent indicated they had no GWG goal and $88.1 \%$ did not discuss a GWG goal with their midwife.

PA is also of interest as it is subject to modification as well as a significant determinant of GWG within the guidelines. In chapter 4 we describe that $51.6 \%$ of the women in our sample reported a reduction in their PA during pregnancy. The highest reduction was reported in sports and brief rigorous activities, but other types of PA were reduced as well. Reduction of PA was mostly seen in women who considered themselves as active before pregnancy, women who experienced pregnancy-related barriers, women who were advised to reduce their PA, and multiparous women. Fewer than 5\% increased their PA. Women who considered themselves as inactive before pregnancy in general did not increase their PA during pregnancy. Pregnancy related barriers were above all tiredness and pain. Women who searched information by themselves were more often continuing their PA. As the intention to have PA seemed higher in women who declined their PA, we conclude that the barriers (pain and tiredness) were preventing them from having physical activity.

Because most women start their pregnancy with a midwife as their care provider, we studied Dutch midwives' behaviours concerning GWG (chapters 5 and 6 ). We found that Dutch midwives varied in their attitude towards engaging in health promotion in general and towards GWG in particular. The time spent on GWG varied, including the time spent on dieteducation and PA-education. Mostly, the advice related to PA was "listen to your body" and "slow down". Midwives varied in their advice on the cut-off points for healthy GWG, and varied in advice about healthy diet and healthy PA. Efforts to promote healthy GWG, including diet-education and PA-education were related to a number of determinants including attitudes, self-efficacy, social influence, the involvement of other health professionals, awareness in their role as health promotor, and perceived barriers, e.g., lack of knowledge, lack of national GWG guidelines, and lack of materials.

In the process of developing the intervention "Come on!" we made several choices regarding the focus (target groups, determinants) of the intervention based on the relevance and expectations regarding change of the most important factors related to GWG (chapter 7). We set a program goal for pregnant women and for midwives and subdivided these in several performance objectives. We crossed the performance objectives with the determinants related to the behaviours and formulated change objectives. After that, we sought methods and strategies to influence the behaviours of pregnant women and midwives. We developed a program including the strategies we choose. We tested elements of the program and prepared the effect-study. In the effect study (chapter 8) we found that the intervention had no effect on diet, PA and gaining weight within the guidelines. However, we confirmed that GWG was independent of pre-pregnancy BMI, and we found that setting explicit goals at the beginning of pregnancy and targeting an adequate GWG helped women to establish healthy GWG. The emails we received during the testing of the intervention indicated that women experienced the internet based program of the intervention as inadequate for their daily practice needs. 


\subsection{Weighing up the findings: putting research into practice}

Not surprisingly, during the process of researching and writing this thesis, I reflected on my own GWG during my two pregnancies, and I talked with other women about their experiences with GWG. Every mother had her own unique tale to tell, and after listening to these different stories, it became clear that there was no common thread binding them together. This anecdotal evidence confirms what we and others have found: GWG is a very complex matter, making it difficult to identify its numerous determinants. ${ }^{6,32,287,291-293}$ This does not mean, however, that there are no strategies for promoting healthy GWG. Our research points to several promising approaches for helping women avoid too little or too much GWG.

\subsubsection{Setting a weight gain goal}

In our cohort study, we found that only $25 \%$ of women in our study set a GWG goal (chapter 3). Based on that, and combined with literature, we included a strategy to set a goal for healthy GWG. Our evaluation of "Come on!" confirmed that setting an adequate GWG goal increased the likelihood of achieving adequate GWG (chapter 8). An important part of our strategy involved counselling pregnant women via an online tailored program, a decision we made in order to avoid overburdening midwives. However, setting a GWG goal might require more face-to-face tailoring than our internet program could offer. For many decisions, pregnant women need proactive and personal communication with a professional to weigh the pros and cons in their own situation. ${ }^{209}$ We asked midwives to discuss GWG goal with pregnant women, but only in the second half of pregnancy. It appears that our intervention did not succeed in finding the balance between the time available in midwifery practices and the need of pregnant women to have early discussions about GWG.

\subsubsection{Every professional is responsible for health}

We were disappointed that our intervention did not succeed in improving the diets and levels of PA in pregnant women and did not increase the number of women with a healthy GWG. But as a result of our study we discovered that midwives need to be made more aware of their role as health promotion professionals and need to be supported in that role. Midwives in our study indicated they are willing to discuss GWG, diet and PA (chapter 5), but - as evidenced by two recent publications showing that effective interventions for encouraging healthy GWG require extensive discussions about GWG and lifestyle $-{ }^{126,294}$ this kind of counselling takes time. Adding one more responsibility to the already overburdened work lives of midwives runs the risk of increasing already high levels of work-related stress. ${ }^{223}$ Further exploration of the balancing act between time available and time required for effective counselling is needed. All professionals need to cooperate in order to find solutions to achieve health for all. 
For example, given their responsibility for creating and maintaining facilities that encourage citizens to be more active, municipalities should join with midwives in finding ways to help pregnant women get sufficient PA. For pragmatic reasons we did not include municipalities in our target group or in the Consortium. In hindsight, their input might be more important than we considered at the start of our project.

\subsubsection{Preventing a decline in physical activity}

Another important finding of our study is the link between a decline in PA and increased GWG. Often, this decline was associated with having received advice to stop PA (chapter 3 ). Midwives can play an important role in helping women to achieve a healthy PA level by confirming the importance of maintaining a sufficient level of PA during pregnancy and by suggesting more convenient and suitable types of PA. Of course, while PA for pregnant women has lots of advantages, it is important to pay special attention to the prevention of injuries. ${ }^{177,187}$ Safe ways to have PA in pregnancy are available, but not always in reach of pregnant women (chapter 4).

The subject PA was introduced - in the latter part of our online intervention, which may have resulted in less attention from our participants. The peer support offered in films was available only with a subsequent log-in and only one film per log-in could be chosen. In retrospect, we suppose that this reduced the attractiveness of the program. In our process evaluation, we need to reflect on other strategies to influence women's PA early in pregnancy.

\subsubsection{Pregnant women first}

We developed the "Come on!" intervention with an online tailored component with the specific intent of saving time for the midwife. We know from qualitative studies among pregnant women, that there is a strong preference for a personal approach with enough time for the delivery of tailored information and client-centred communication. ${ }^{209,295}$ Midwives are ideally positioned to provide that kind of care, encouraging women to achieve a lifestyle that promotes and protects their health and the health of their children.

Maternity care providers like midwives are the obvious and crucial element for promoting healthy GWG in pregnant women. We asked midwives to discuss GWG and lifestyle with their clients, but we did not check to see if midwives were able to find our time to have those discussions. In their everyday practice, they may have had priorities other than GWG. Our training session was designed to increase midwives' awareness of the value of health promotion, but we do not know if only one session was sufficient to change their behaviour. A recently published study, examined the language used by midwifery professional bodies, and identified three types of scope of practice; 1 ) a formal type focusing on midwifery practice, 2 ) a less formal type that focused on the midwife as agent, and 3) a type which featured the woman as agent. ${ }^{296}$ Reflecting on our choice, we realize that we too used a midwife centred approach instead of one that was client centred. As a result, our message to midwives was: 1) we built an intervention to save your time, 2) we want you to spend a small 
amount of time to discuss GWG and lifestyle with pregnant women, and 3) we understand and agree that you will do this in the second half of pregnancy. Had we also listened to pregnant women our message to midwives would have been: 1) women need time at the beginning of pregnancy to discuss GWG and lifestyle with you, 2) we need you to look for opportunities to do this; and 3) can we help you with that.

\subsection{Limitations and strengths}

Our studies had several limitations. First, our samples were not representative of the Dutch population of pregnant women and midwives. Women with low incomes, low levels of education and ethnic minorities were underrepresented, and, compared to the population of pregnant women in the Netherlands, we had more nulliparous women, more women with a normal BMI and fewer women who were classified as overweight. As these characteristics influence GWG, care should be taken when generalising our results to a larger population. The same is true for our sample of midwives: because it is a self-selected sample, it is likely that these midwives have a higher level of interest in GWG than other midwives in the Netherlands.

We started our studies with the use of the ASE-Model (attitude, social influence, self-efficacy), a widely accepted method for explaining conscious behaviour. While the ASE-model is still used by health researchers, ${ }^{297}$ the developer of that model has expanded the approach, creating the "Integrated change model" (I-Change model). ${ }^{298}$ In this approach, the ASE-model is combined with other theories (e.g., Trans Theoretical Theory, Goal Setting Theory, Health Belief Model, and Social Cognitive Theory) into an integrated framework. Several researchers have used the I-change model, ${ }^{299-303}$ and, in our study, we called on most of these other theories to guide the development of our intervention. We did not, however, use the specific I-change model to evaluate our initial surveys. Had we done that, we may have altered items in our questionnaires, resulting in the collection of information valuable for developing our intervention.

Another limitation is our use of non-validated instruments, - with the sole exception of the Food Frequency Questionnaire. Although we took steps to increase the content validity of the questionnaires by involving pregnant women, students, and midwives, in formulating the questions, we remain unsure about the test validity and the construct validity. We had little choice, however. This is a relatively new area of interest in midwifery science, and as such there were no validated surveys available when we did our research. Furthermore, we relied in self-reported of weight and PA, which limits our ability to compare our findings with findings of others.

As noted earlier, a considerable part of the counselling of pregnant women in "Come on!" was done via an online tailored program - a decision we made in order to relieve midwives of this task. It may be that an online program is less successful in helping women set a clear GWG-goal and in creating a positive attitude towards this goal. Time pressure prevented us from making adjustments in our online program (even after we got feedback from pregnant 
women and students) and limited the time we had to train midwives in motivational interviewing and shared decision making.

Furthermore, we do not know if women who participated in the first part of the program, i.e., the control group, were more aware of the topic GWG and discussed this more often with their midwives. It is possible that the "Hawthorne effect" of being in the study resulted in more women having a healthy GWG. If this was the case - that women in the control group were more likely to have a healthy GWG as compared to Dutch women overall - it would be more difficult to show an effect of the intervention.

Midwives who participated in the evaluation of "Come on!", may have had more positive attitudes towards promoting GWG, which may have influenced their performances. However, as we used an historical cohort in the intervention study, where each midwifery practice was included in both the control and intervention cohort, we controlled for the possible overrepresentation of midwives with a positive attitude.

In this thesis, we used a variety of research methods to study different aspects of GWG. Combining a systematic literature review, quantitative and qualitative research provided us with a broad and deep view of the subject. We used the Intervention Mapping (IM) protocol to systematically approach the problem of unhealthy GWG from different perspectives.

Although we found IM to be useful, it was also time-consuming. IM is typically applied to unidimensional behaviours and can become unwieldly when applied to complex behaviours such as GWG. ${ }^{279}$ As we worked with IM, we often made choices based on relevance, expectations of change, and feasibility. Looking back, and considering the results of "Come on!", some of these choices may be questioned. What follows is a discussion of the three most important choices we made and the consequences of those choices, including (1) the selection of target groups, (2) the factors related to GWG, and (3) the selection of GWG guidelines we applied.

\subsubsection{Target groups}

The first choice was to focus on healthy pregnant women and their midwives. We used a pragmatic definition of healthy pregnant women: "women receiving care by a primary care midwife, including women in all pre-pregnancy BMI-groups". Using a public health perspective, we assumed that also for this healthy target group health benefits are within reach. ${ }^{304}$ We were not excluding unhealthy pregnant women per se, but wanted to prevent bias in our research by including women with (pregnancy related) health problems. In all of the pre-pregnancy BMI groups there were some healthy women with an unhealthy GWG (chapter 5 and 8).

It seems obvious to target pregnant women in order to establish healthy GWG, as gestational weight gain can only occur during pregnancy. However, starting before women become pregnant may add to the success of an intervention, ${ }^{210,304}$ especially because GWG in early pregnancy is related to GWG at the end of pregnancy. ${ }^{254,305}$ In early pregnancy, women may already be considered as having an unhealthy GWG, before they even see a health professional. ${ }^{254}$ The pre-conceptional period has often been mentioned as important in helping women to achieve a healthy lifestyle with the aim of improving the health of women 
and their babies during pregnancy and thereafter. ${ }^{210,304,306}$ For example, pre-conception care will allow special attention to the importance of the continuation of PA (chapter 6). Telling women in the pre-conception period that they can continue their PA level once they are pregnant, may prevent a reduction of PA in early pregnancy.

Primary care midwives, our second target group, are in the position to be able to reach healthy pregnant women. In the Netherlands, almost all women (85\%) start their pregnancy with a primary care midwife. ${ }^{143}$ Midwives are educated to distinguish normal pregnancy from pathological pregnancy. Their knowledge of physiology in pregnancy and childbirth, and their core competence of promoting physiology in pregnancy and childbirth prevent many interventions. ${ }^{210,307}$ Midwives are an important target group for the establishment of a healthy environment for the pregnant woman. ${ }^{296}$ However, midwives' awareness of their role in health promotion and the experiences they have had in promoting health is an important determinant for their behaviour in promoting healthy GWG (chapter 3). Despite the fact that positive effects of "Come on!" were not evident in our study, we stand by our choice to focus on midwives in our intervention. However, we learned that midwives need more skills and more time to take advantage of this particular midwifery role.

Other target groups: We know that the problems of individuals have environmental causes. ${ }^{62}$, ${ }^{308}$ The research of Alice van der Pas and Hoek, for example, shows how lack of societal support for parents can increase levels of child abuse. ${ }^{309,310}$ Seen from this perspective, targeting unhealthy GWG in isolation from the context in which it occurs is less likely to succeed than a multi-dimensional intervention with a larger goal of creating a world where children can grow up in health as defined by WHO. ${ }^{311}$ Thus, the successful promotion of healthy GWG must target policy makers, the partners and parents of pregnant women, and society in general. Each of these groups are part of the context in which pregnant women and midwives live and work.

The best way to promote health is to invest in caregivers and public health interventions, ${ }^{296}$ which our intervention did. However, in retrospect, we see that it is necessary to enlist a broader range of target groups. Future efforts to encourage healthy GWG will be more effective if they take the consideration of the larger environment of expecting mothers.

\subsubsection{Factors related to healthy gestational weight gain}

Our second choice was to focus on setting an adequate GWG goal and on diet and PA as key factors related to GWG in healthy pregnant women (chapter 3). In our study of healthy pregnant women (chapter 3), we were able to explain $15 \%$ of the variance in GWG as measured using the IOM-guidelines. We found that diet (measured in consumption of vegetables, fruit and fish) was not significantly associated with healthy GWG; we also found that a decrease in PA was one of the factors related to GWG above the IOM-guidelines. Based on our review of intervention studies (chapter 2) -which confirmed that diet could help obese women to have a lower GWG - we considered healthy diet a possible determinant for healthy GWG in healthy women as well (chapter 7). We identified a variety of determinants of 
behaviours related to GWG and used theoretical models and theory-based applications to influence these determinants.

Given that we found no clear relationship between diet and healthy GWG, it is useful to consider other factors that may be related to GWG. Recent research suggests that it may be time to alter conventional approaches to the problem of unhealthy GWG. Hill et al. provided a conceptual model of the psycho-social determinants contributing to GWG, including body perception and self-esteem. ${ }^{43}$ Kapadia et al. built on this model, creating a so-called "pinwheel" of psychological factors that are associated with GWG. ${ }^{29}$ Another possible influence on GWG is described in a report by the WHO that warns about the contamination of food with endocrine disrupters. ${ }^{312}$ Studies have shown the possible negative effects of these chemicals on unborn babies and on the endocrine system of pregnant women. ${ }^{312-316}$ Moreover, these chemicals appear to be associated with GWG. ${ }^{317}$ A third new area that may offer new approaches to GWG is found in research on microbiomes in pregnant women. ${ }^{318}$ The human gastrointestinal tract is host to a vast ecosystem of microbes which are necessary for health. ${ }^{319}$ Specific alterations in the microbiome have the potential to influence GWG and increase susceptibility to other diseases including obesity. ${ }^{320-322}$ However, a broadened approach at the same time will further expand the complexity of GWG.

\subsubsection{Selected guidelines for gestational weight gain}

Our third choice was the selection of the Institute of Medicine (IOM)-guidelines of 2009 as a reference for healthy GWG. These were the best available and most widely used guidelines on GWG at the start of our study. ${ }^{6}$ Since 2009, studies have confirmed that women who have a GWG above the IOM-guidelines have higher risks for poor outcomes. ${ }^{323-327}$ However, there is criticism of the cut-off points defining GWG in these guidelines, including from the authors of the guidelines themselves, who stated that these cut-off points were not yet evidence based (p 254):

"The committee chose to formulate the new (2009) guidelines with a range for each category of pre pregnant Body Mass Index (BMI). This range reflects the impression of the estimates on which these recommendations are based, the reality that good outcomes are achieved with a range of weight gains, and the many additional factors that may need to be considered when making a recommendation for an individual woman."

In the literature, debates are ongoing with regard to the precise cut-off points, particularly for obese women. One study, done in a healthy Dutch population, compared the IOM-guidelines to a GWG cut-off point of $15 \mathrm{~kg}$ (irrespective the pre-pregnancy BMI category of the woman) and found that the latter was more accurate in predicting referrals during pregnancy from midwife-led care to specialized obstetric-led care. ${ }^{328}$ However, these researchers did not relate GWG with long term outcomes of overweight or obesity for women or babies. Savitz et al. demonstrated that regarding Caesarean section, large for gestational age (Iga) babies, 
small for gestational age (sga) babies and preterm births, the optimum GWG was 10 to $14 \mathrm{~kg}$ for all pre-pregnancy BMI groups. ${ }^{329}$ Cedergren et al. performed a population based cohort study among pregnant women in Sweden and related their GWG to various pregnancy outcomes. They found that the optimum of GWG in relation to these outcomes was far less than the IOM-guidelines recommend. Their conclusion was that underweight women should gain 4 to $10 \mathrm{~kg}$ (IOM 12.5 to 18.0), normal weight women 2 to $10 \mathrm{~kg}$ (IOM 11.5-16.0), overweight women less than $9 \mathrm{~kg}$ (IOM 7.0 to 11.5) and obese women less than $6 \mathrm{~kg}$ (IOM 5.0 to 9.0$).{ }^{330}$ Based on obstetric outcomes of GWG research in obese women, some authors recommend adaptation of the IOM-guidelines for women with severe obesity, in such a way that these women should gain zero kg during pregnancy. ${ }^{331,332}$ A review and meta-analysis of cohort studies on adverse outcomes in severely obese women concluded that a GWG below the guidelines might be appropriate for certain women. ${ }^{333}$

Another problem with the IOM-guidelines is related to the boundary between normal weight and overweight. Suppose two sisters, both with a height of 1.73 meters get pregnant. One has a weight of $74.6 \mathrm{~kg}$, so her BMI is $24.9 \mathrm{~kg} / \mathrm{m}^{2}$ and she will be advised to gain within the range of 11.5 to $16 \mathrm{~kg}$. Her sister, with a weight of just hundred grams more $(74.7 \mathrm{~kg})$ has a BMI of $25.0 \mathrm{~kg} / \mathrm{m}^{2}$ and will be advised to gain 7 to $11.5 \mathrm{~kg}$. Figure 9.1 makes this problem visible. Intuitively, recommendations for GWG should use BMI measured at a continuous level instead of using the four ordinal level BMI-categories (underweight, normal weight, overweight and obese). This problem is inadequately discussed in the literature.

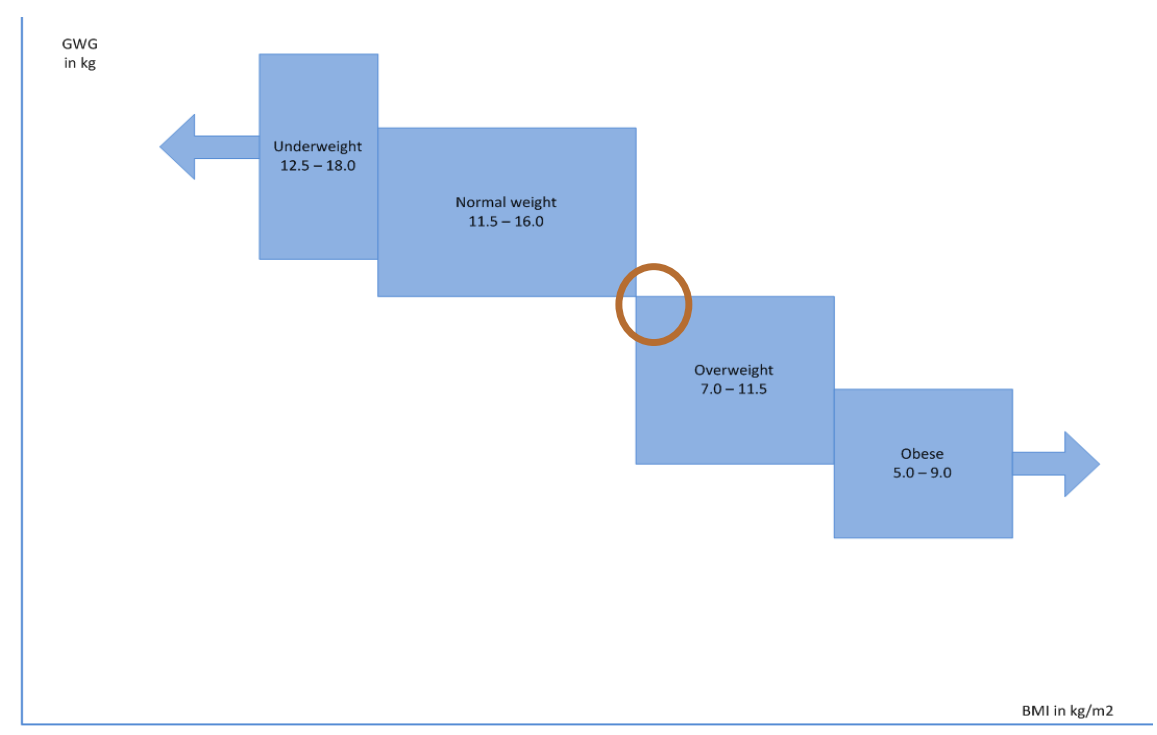

Figure 9.1 Figure of recommendations for gestational weight gain (GWG) for different Body Mass Index (BMI) groups according to the 2009 Institute of Medicine (IOM) guidelines.

The orange circle points at the sudden break in the IOM-guidelines, women at the end of the "normal weight" BMI group are recommended to have a GWG between 11.5 and $16.0 \mathrm{~kg}$, while women at the beginning of the "overweight" BMI group are advised to have a GWG between 7.0 and $11.5 \mathrm{~kg}$ Abbreviations: BMI, Body Mass Index; GWG, gestational weight gain; IOM, Institute of Medicine 
A group of researchers proposed providing cut-off points based on Z-score charts for the relation between BMI and GWG, an approach they believe to have the potential to guide women toward a GWG better related to healthy outcomes. ${ }^{334,335}$ The use of Z-scores allows advice for GWG cut off points to be tailored to actual BMI and not a BMI category, providing a target GWG that fits better with pregnant women's own personal situation.

In our study, as in other studies, ethnicity was a factor influencing GWG (chapter 5). ${ }^{56,188}$ Given that the "modal" woman in Scandinavian countries tends to be taller and heavier than the "modal" woman in Asiatic countries, it is reasonable that every country, or perhaps every ethnic group have its own GWG guideline. Ethnic differences has been addressed in the IOMguidelines, albeit specified in three subtypes (Hispanic, Non-Hispanic White and Non-Hispanic Black). ${ }^{6}$

Considering the limitations associated with the use of the cut-off points specified in the IOMguidelines, we question their rigid use. Research leads us to believe that cut-off points associated with a GWG guideline should not be strictly followed, especially with BMIs close to $25 \mathrm{~kg} / \mathrm{m}^{2}$. Furthermore, it is likely that the use of different cut-off points, or the use of standard scores for pre-pregnancy BMIs (instead of categories) would result in fewer women would be classified as gaining "too little" or "too much" weight, reducing the large proportion of the women who gain weight outside the IOM-guidelines. In our effect study, we do not know whether we would have an effect of the intervention when we had used other guidelines.

\subsection{Recommendations for practice}

Our research confirms that women should be encouraged to continue or increase their PA during pregnancy. Caregivers need to discuss this issue - more extensively than in "Come on!" - in person with each woman. Other strategies to influence pregnant women's PA might be explored as well.

Antenatal care givers should also encourage women to set a goal for GWG that is appropriate for them, using GWG recommendations based on existing guidelines and current research. As our research has shown, exact evidence-based cut-off points cannot be provided yet; so it falls to the caregiver to weigh the evidence and the situation of her client.

Finally, our research points to the fact that these recommendations are more likely to be followed when midwives have awareness of their role in health promotion. Strategies to influence midwives' awareness might be further expanded, for instance with short educational films.

In order to help women to achieve healthy lifestyle goals, they need to be in the centre of care. Researchers need to increase the cooperation with pregnant women. Midwives may benefit from applying methods of communicating lifestyle behaviour, such as motivational interviewing and shared decision making. ${ }^{209,295,336,337}$ As developing these skills need time and investment, we recommend training these skills to be included in midwifery education. 


\subsection{Recommendations for further research}

In future research, our team needs to further explore the experiences of users of the "Come On!" intervention by means of a process evaluation. Further analysis of our data will allow us to find the weak links in the program and discover where improvements are necessary. The change objectives formulated during the Intervention Mapping process can easily be used to guide this research. As minor changes can make huge differences, it is possible that with small adaptations in the training and the internet program, more positive outcomes may be achieved.

More generally, GWG researchers need to find consensus on the evidence based cut-off points for healthy GWG. Analysing the data of healthy women is necessary to achieve this goal. A promising initiative in this respect is the International Weight Management in Pregnancy (i-WIP) Collaborative Group, which is cooperating on a project developing a large database (over 9,000 healthy and unhealthy women) of individual patient data on the determinants and outcomes related to GWG. ${ }^{338}$ Another, more local initiative is VeCaS, a research project in which data from 25 midwifery practices in the Netherlands are collated. ${ }^{339}$ In this study, data, including weights from all antenatal consultations of healthy pregnant women, are gathered to be used in future research. ${ }^{339}$ It is important to invest in defining healthy GWG for different populations because this will allow caregivers to more effectively advise women based on their personal characteristics and not broad generalisations. Besides studying GWG of healthy women in general, it is worthwhile to study GWG of women with lower levels of education, women with lower incomes, women who experienced "hardship" (defined as lacking basic necessities like food, rent or medical care) in childhood, and women suffering intimate partner violence, because all of these conditions are associated with too high or too low GWG ${ }^{49-51,54,55}$ Although medical conditions occur more often in these groups, the characteristics mentioned are not an indication per se to consider these women as unhealthy, which makes them a group in reach of midwives. More research is necessary to find out why these characteristics are associated with too high or too low GWG and what the prenatal care provider can do to promote healthy GWG.

Furthermore we need to continue the study of interventions for the promotion of healthy GWG. Investing in prenatal care by midwives is a promising strategy. Although it will cost money, reducing the "normpraktijk" will give midwives the time they need to invest in discussions with pregnant women about setting an appropriate GWG-goal and helping women to maintain or increase their physical activity during pregnancy. Investment in the health of pregnant women will in the long run, reduce the costs of maternity care and improve the health of women and babies in later life.

We have weighed the value, the strengths, and the limitations of our work. The goal of our research was to help more women find the balance between too high and too low GWG, and to support midwives in the work of encouraging women to find their healthy GWG. Using common sense in setting a GWG goal, being physically active, and further investment in personal prenatal care by midwives will move us closer to the goal we seek 


\section{Large tables and figure $\mathbf{2 . 2}$}


Table 2.1 Quality assessment and characteristics Randomised Controlled Trials (RCT's)

\begin{tabular}{|c|c|c|c|c|c|c|}
\hline $\begin{array}{l}\text { Author and } \\
\text { year } \\
\text { intervention in } \\
\text { short }\end{array}$ & Setting & $\begin{array}{l}\text { Characteristics of } \\
\text { study-population on } \\
\text { baseline }\end{array}$ & Definitions & Intervention & PEDro score & Quality Assessment \\
\hline $\begin{array}{l}\text { Asbee et al., } \\
2009^{88} \\
\text { Dietary and } \\
\text { lifestyle } \\
\text { counselling for } \\
\text { all BMI }\end{array}$ & $\begin{array}{l}\text { North } \\
\text { Carolina, USA } \\
\text { October } 2005 \\
\text { - April } 2007\end{array}$ & $\begin{array}{l}100 \text { randomised } \\
57 \text { intervention group } \\
43 \text { control group } \\
\text { age: } 26.7 \text { vs } 26.4 \text { yr } \\
\text { race: } 56 \% \text { Hispanic, } \\
24 \% \text { African American; } \\
13 \% \text { White; } 7 \% \text { Other } \\
\text { education: unclear } \\
\text { SES: NR } \\
\text { PA: NR } \\
\text { diet: NR } \\
\text { parity: } 45 \% \text { first preg } \\
\text { pre pregn weight: } \\
64.86 \text { vs } 64.95 \text { kg } \\
\text { weight: NR } \\
\text { height: } 160 \text { vs } 160 \mathrm{~cm} \\
\text { BMI: } 25.5 \text { vs } 25.6 \\
\text { kg/m2 } \\
\text { gestational age at } \\
\text { start: } 13.7 \text { vs } 13.6 \text { wks }\end{array}$ & $\begin{array}{l}\text { GWG: unclear } \\
\text { BMI: self- } \\
\text { reported pre } \\
\text { pregnancy } \\
\text { weight and } \\
\text { height } \\
\text { guidelines: } \\
\text { IOM } 1990 \\
\text { UW + NW: }<26 \\
\text { OW: } 26-29 \\
\text { OB: }>29-40\end{array}$ & $\begin{array}{l}\text { 1. standardised counselling } \\
\text { session with dietician with } \\
\text { information on pregnancy- } \\
\text { specific dietary and lifestyle } \\
\text { choices; } 40 \% \text { carbohydrates, } \\
30 \% \text { protein and } 30 \% \text { fat } \\
\text { stimulation to moderate } \\
\text { intensity exercise at least } 3 \\
\text { times a week } \\
\text { feedback on weight gain } \\
\text { 2. marginal counselling on diet } \\
\text { and exercise, weight } \\
\text { measuring and reporting }\end{array}$ & $\begin{array}{l}\text { eligibility criteria specified + } \\
\text { randomisation + } \\
\text { allocation concealed + } \\
\text { baseline similar + } \\
\text { blinding of subjects - } \\
\text { blinding of therapists - } \\
\text { blinding of assessors - } \\
\text { at least } 85 \%+ \\
\text { intention to treat analyses - } \\
\text { reporting with statistical } \\
\text { comparison + } \\
\text { point measures AND measures } \\
\text { of variability - } \\
\text { PEDro Sum score } 6\end{array}$ & $\begin{array}{l}\text { randomisation: computer } \\
\text { generated (before or after } \\
\text { inclusion?) } \\
\text { allocation: opaque } \\
\text { envelops } \\
\text { personnel: NR } \\
\text { assessors: NR } \\
\text { adherence: } 100 / 100= \\
100 \%\end{array}$ \\
\hline $\begin{array}{l}\text { Barakat et al., } \\
2009^{90} \\
\text { Resistance } \\
\text { exercise training } \\
\text { for all BMI with } \\
\text { low SES }\end{array}$ & $\begin{array}{l}\text { primary care } \\
\text { Madrid, Spain } \\
\text { Jan } 2000- \\
\text { March } 2002\end{array}$ & $\begin{array}{l}160 \text { randomised } \\
72 \text { intervention group } \\
70 \text { control group } \\
\text { GDM: } 23.6 \% \text { vs } 31.4 \% \\
\text { age: } 30.4 \text { vs } 29.5 \\
\text { race: Caucasian } \\
\text { education: < high } \\
\text { school: } 34.7 \% \text { vs } \\
44.3 \% ; \text { high school: } \\
38.9 \% \text { vs } 42.9 \% ;>\text { high } \\
\text { school: } 26.4 \% \text { vs } 12.9 \% \\
\text { SES: } 100 \% \text { low to }\end{array}$ & $\begin{array}{l}\text { GWG: unclear } \\
\text { BMI: weight } \\
\text { and height at } \\
\text { start of study } \\
\text { UW: }<18.5 \\
\text { NW: } 18.5- \\
24.9 \\
\text { OW: } 25-29.9 \\
\text { OB: }>30\end{array}$ & $\begin{array}{l}\text { 1. women had } 3 \text { sessions a } \\
\text { week a } 35-40 \text { min for } 26 \\
\text { weeks training; heart rate < } \\
80 \% \text { of } 220 \text { bpm - age; toning } \\
\text { and resistance; number of } \\
\text { women in group NR; } \\
\text { supervision NR } \\
\text { 2. women were asked to } \\
\text { maintain their level of activity }\end{array}$ & $\begin{array}{l}\text { eligibility criteria specified + } \\
\text { randomisation }+ \\
\text { allocation concealed + } \\
\text { baseline similar + } \\
\text { blinding of subjects - } \\
\text { blinding of therapists - } \\
\text { blinding of assessors + } \\
\text { at least } 85 \%+ \\
\text { intention to treat analyses + } \\
\text { reporting with statistical } \\
\text { comparison + } \\
\text { point measures AND measures }\end{array}$ & $\begin{array}{l}\text { randomisation: "allocation } \\
\text { concealment process" } \\
\text { allocation: blind } \\
\text { personnel: NR } \\
\text { assessors: blinded } \\
\text { adherence: } 142 / 160=89 \%\end{array}$ \\
\hline
\end{tabular}




\begin{tabular}{|c|c|c|c|c|c|c|}
\hline & & $\begin{array}{l}\text { medium: income } \\
\text { individual < } 16.000 € \\
\text { or family < } 23.000 € \\
\text { PA: sedentary job: } \\
36.1 \% \text { vs } 30.0 \% \\
\text { diet: NR } \\
\text { parity: } 65 \% \text { first preg } \\
\text { pre pregn weight: NR } \\
\text { weight: } 64.7 \text { vs } 60.1 \mathrm{~kg} \\
\text { (pre or at start?) } \\
\text { height: } 163 \text { vs } 160 \mathrm{~cm} \\
\text { BMI: } 24.3 \text { vs } 23.4 \\
\text { kg/m2 } \\
\text { gest age at start: NR }\end{array}$ & & & $\begin{array}{l}\text { of variability - } \\
\text { PEDro Sum score } 8\end{array}$ & \\
\hline $\begin{array}{l}\text { Bechtel- } \\
\text { Blackwell } 2003 \\
100 \\
\text { Nutritional } \\
\text { group sessions } \\
\text { for African } \\
\text { American } \\
\text { adolescents }\end{array}$ & $\begin{array}{l}\text { USA } \\
\text { period of } \\
\text { enrolment: } \\
\text { NR }\end{array}$ & $\begin{array}{l}60 \text { randomised } \\
30 \text { intervention group } \\
30 \text { control group } \\
\text { age: } 13-18 \text { yr } \\
\text { race: African-American } \\
100 \% \\
\text { education: NR } \\
\text { SES: NR } \\
\text { PA: NR } \\
\text { diet: NR } \\
\text { parity: NR } \\
\text { pre pregn weight: NR } \\
\text { weight: NR } \\
\text { height: NR } \\
\text { BMI: NR } \\
\text { gest age at start: NR } \\
\end{array}$ & $\begin{array}{l}\text { GWG: NR } \\
\text { BMI: NR }\end{array}$ & $\begin{array}{l}\text { 1. } 3 \text { times } 20 \text { minutes group } \\
\text { sessions that address } \\
\text { nutritional needs specific to } \\
\text { the woman's stage of their } \\
\text { pregnancy and Computer } \\
\text { Assisted Self Interview } \\
\text { 2. nutritional consult by a } \\
\text { dietician at least once during } \\
\text { pregnancy }\end{array}$ & $\begin{array}{l}\text { eligibility criteria specified - } \\
\text { randomisation - } \\
\text { allocation concealed - } \\
\text { baseline similar - } \\
\text { blinding of subjects - } \\
\text { blinding of therapists - } \\
\text { blinding of assessors - } \\
\text { at least } 85 \% \text { - } \\
\text { intention to treat analyses + } \\
\text { reporting with statistical } \\
\text { comparison + } \\
\text { point measures AND measures } \\
\text { of variability - } \\
\text { PEDro Sum score } 2\end{array}$ & $\begin{array}{l}\text { randomisation: by } \\
\text { "selecting every other } \\
\text { name at a random starting } \\
\text { point" } \\
\text { allocation NR } \\
\text { personnel: unclear } \\
\text { assessors: NR } \\
\text { adherence: } 46 / 60=77 \% \\
\text { or } 35 / 60=58 \%\end{array}$ \\
\hline $\begin{array}{l}\text { Cavalcante et } \\
\text { al., } 2009^{91} \\
\text { Water aerobics } \\
\text { for all BMI with } \\
\text { sedentary } \\
\text { lifestyle }\end{array}$ & $\begin{array}{l}\text { low-risk } \\
\text { Campinas, } \\
\text { Brazil } \\
\text { March } 2002- \\
\text { November } \\
2008\end{array}$ & $\begin{array}{l}71 \text { randomised } \\
34 \text { intervention group } \\
\text { ( } 33 \text { analysed) } \\
37 \text { control group } \\
\text { GDM: NR } \\
\text { age: } 25.8 \text { vs } 24.4 \mathrm{yr} \\
\text { race: NR } \\
\text { education: only }\end{array}$ & $\begin{array}{l}\text { GWG: unclear } \\
\text { BMI: unclear }\end{array}$ & $\begin{array}{l}\text { 1. regular and moderate } \\
\text { practice of water aerobics for } \\
50 \text { minutes three times a week } \\
\text { in indoor swimming pool } 28- \\
30 \text { degrees, } 70 \% \text { of predicted } \\
\text { max heart rate; trained } \\
\text { instructor; costs were paid; } \\
\text { number of women in group }\end{array}$ & $\begin{array}{l}\text { eligibility criteria specified - } \\
\text { randomisation }+ \\
\text { allocation concealed + } \\
\text { baseline similar + } \\
\text { blinding of subjects - } \\
\text { blinding of therapists - } \\
\text { blinding of assessors + } \\
\text { at least } 85 \%+\end{array}$ & $\begin{array}{l}\text { randomisation computer } \\
\text { generated } \\
\text { allocation: opaque } \\
\text { envelops } \\
\text { personnel: blinded } \\
\text { assessors: blinded } \\
\text { adherence: } 70 / 71=99 \%\end{array}$ \\
\hline
\end{tabular}




\begin{tabular}{|c|c|c|c|c|c|c|}
\hline & & $\begin{array}{l}\text { primary school: } 47.1 \% \\
\text { vs } 27.0 \% \\
\text { SES: NR } \\
\text { PA: sedentary: not } \\
\text { doing regular exercises } \\
\text { diet: NR } \\
\text { parity: } 47.1 \% \text { vs } 62.2 \% \\
\text { pre pregn weight: NR } \\
\text { weight: } 63.8 \text { sd } 12.7 \text { vs } \\
60.8 \text { sd } 10.2 \\
\text { height: NR } \\
\text { BMI: } 24.1 \text { vs } 23.4 \\
\text { kg/m2 } \\
\text { gest age at start: NR }\end{array}$ & & $\begin{array}{l}\text { NR } \\
\text { 2. no physical exercise during } \\
\text { pregnancy }\end{array}$ & $\begin{array}{l}\text { intention to treat analyses + } \\
\text { reporting with statistical } \\
\text { comparison + } \\
\text { point measures AND measures } \\
\text { of variability + } \\
\text { PEDro Sum score } 8\end{array}$ & \\
\hline $\begin{array}{l}\text { de Barros et al., } \\
2010 \\
89 \\
\text { Resistance } \\
\text { elastic band } \\
\text { exercise with } \\
\text { coaching and } \\
\text { glycaemic } \\
\text { control for GDM }\end{array}$ & $\begin{array}{l}\text { Sao Paula, } \\
\text { Brazil } \\
\text { October } 2006 \\
\text { - November } \\
2008\end{array}$ & $\begin{array}{l}64 \text { randomised } \\
32 \text { intervention group } \\
32 \text { control group } \\
\text { GDM: } 100 \% \\
\text { age: } 31.8 \text { vs } 32.4 \mathrm{yr} \\
\text { race: NR } \\
\text { education: NR } \\
\text { SES: NR } \\
\text { PA: sedentary } \\
\text { diet: NR } \\
\text { parity: NR } \\
\text { pre pregn weight: NR } \\
\text { weight: NR } \\
\text { height: NR } \\
\text { BMI: } 25.3 \text { vs } 25.4 \\
\text { kg/m2 } \\
\text { gest age at start: } 31.6 \\
\text { vs } 31.1 \text { wk }\end{array}$ & $\begin{array}{l}\text { GWG: unclear } \\
\text { BMI: unclear } \\
\text { GDM: OGTT } 3 \\
\text { hr with } 100 \mathrm{~g} \\
\text { glucose or } 2 \mathrm{hr} \\
\text { OGTT with } 75 \mathrm{~g} \\
\text { glucose; cut of } \\
\text { point unclear } \\
\text { sedentary: } \\
\text { International } \\
\text { Physical } \\
\text { Activity } \\
\text { Questionnaire; } \\
\text { cut of point } \\
\text { unclear }\end{array}$ & $\begin{array}{l}\text { All: Instructions regarding } \\
\text { glucose self-monitoring to } \\
\text { maintain fasting glucose }<95 \\
\mathrm{mg} / \mathrm{dL} \text { or postprandial }<120 \\
\mathrm{mg} / \mathrm{dL} \text {; systematically } \\
\text { evaluation and dietary } \\
\text { instructions from a } \\
\text { nutritionist; } 7 \text { servings } \\
\text { 1. Resistance Exercise with } \\
\text { elastic band; } 90 \text { min after } \\
\text { meal; Intensity controlled and } \\
\text { increased; } 1 \text { time/wk together } \\
\text { with researcher and } 2 \\
\text { times/wk at home; } 1 / \text { wk } \\
\text { telephone contact } \\
\text { 2. routine care as above }\end{array}$ & $\begin{array}{l}\text { eligibility criteria specified + } \\
\text { randomisation }+ \\
\text { allocation concealed + } \\
\text { baseline similar }+ \\
\text { blinding of subjects - } \\
\text { blinding of therapists + } \\
\text { blinding of assessors + } \\
\text { at least } 85 \%+ \\
\text { intention to treat analyses + } \\
\text { reporting with statistical } \\
\text { comparison }+ \\
\text { point measures AND measures } \\
\text { of variability + } \\
\text { PEDro Sum score } 10\end{array}$ & $\begin{array}{l}\text { randomisation computer } \\
\text { generated } \\
\text { allocation opaque } \\
\text { envelops } \\
\text { personnel: } \\
\text { blinded } \\
\text { assessors blinded } \\
\text { adherence } 64 / 64=100 \%\end{array}$ \\
\hline $\begin{array}{l}\text { Guelinckx et al., } \\
2010 \\
92 \\
\text { Lifestyle } \\
\text { intervention for }\end{array}$ & $\begin{array}{l}\text { University } \\
\text { hospitals } \\
\text { Leuven } \\
\text { Belgium } \\
1 \text { March } 2006 \\
\text { - } 31 \text { January } \\
\end{array}$ & $\begin{array}{l}195 \text { randomised into } 3 \\
\text { groups } \\
65 \text { active intervention } \\
\text { group } \\
65 \text { passive intervention } \\
\text { group }\end{array}$ & $\begin{array}{l}\text { GWG: weight } \\
\text { in delivery } \\
\text { room before } \\
\text { delivery minus } \\
\text { self reported } \\
\text { pre pregnancy }\end{array}$ & $\begin{array}{l}\text { 1. } 3 \text { one hour sessions }(15,20 \\
\text { and } 32 \text { wks) in group of max } 5 \\
\text { women, with trained } \\
\text { nutritionist, healthy eating, } \\
\text { reading nutrition fact labels, } \\
\text { increasing PA, behavioural }\end{array}$ & $\begin{array}{l}\text { eligibility criteria specified + } \\
\text { randomisation - } \\
\text { allocation concealed - } \\
\text { baseline similar + } \\
\text { blinding of subjects - } \\
\text { blinding of therapists - }\end{array}$ & $\begin{array}{l}\text { randomisation: NR } \\
\text { allocation: NR } \\
\text { personnel: NR } \\
\text { assessors: medical records } \\
\text { and self-reporting } \\
\text { questionnaires }\end{array}$ \\
\hline
\end{tabular}




\begin{tabular}{|c|c|c|c|c|c|c|}
\hline $\mathrm{OB}$ & 2008 & $\begin{array}{l}65 \text { control group } \\
\text { GDM: NR } \\
\text { age: } 28.0 \text { vs } 28.7 \text { vs } \\
29.4 \text { yrs } \\
\text { race: Caucasian } 100 \% \\
\text { education: NR } \\
\text { SES: NR } \\
\text { PA: NR } \\
\text { diet: vitamin use: } \\
81.4 \% \text { vs } 76.5 \% \text { vs } \\
85.4 \% \\
\text { parity: first pregnancy: } \\
7.1 \% \text { vs } 5.4 \% \text { vs } 16.3 \% \\
\text { pre pregn weight: } 93.2 \\
\text { vs } 92.8 \text { vs } 90.3 \mathrm{~kg} \\
\text { weight: NR } \\
\text { height: } 165 \text { vs } 167 \text { vs } \\
164 \mathrm{~cm} \\
\text { BMI: } 34.1 \text { vs } 33.4 \text { vs } \\
33.5 \mathrm{~kg} / \mathrm{m} 2 \\
\text { gest age at start: } 9.3 \\
\text { vs } 10.2 \text { vs } 10.2 \text { wk }\end{array}$ & $\begin{array}{l}\text { weight } \\
\text { BMI: self } \\
\text { reported pre } \\
\text { pregnancy } \\
\text { weight and } \\
\text { measured } \\
\text { height at first } \\
\text { visit } \\
\text { guidelines: } \\
\text { IOM } 1990 \text { OB } \\
\text { upper limit } \\
11.2 \mathrm{~kg}\end{array}$ & $\begin{array}{l}\text { modification brochure as } \\
\text { group } 2 \\
\text { 2. brochure with advice on } \\
\text { nutrition and PA and tips to } \\
\text { limit GWG } \\
3 \text {, routine prenatal care }\end{array}$ & $\begin{array}{l}\text { blinding of assessors }+ \\
\text { at least } 85 \% \text { - } \\
\text { intention to treat analyses + } \\
\text { reporting with statistical } \\
\text { comparison + } \\
\text { point measures AND measures } \\
\text { of variability - } \\
\text { PEDro Sum score } 5\end{array}$ & adherence $122 / 195=63 \%$ \\
\hline $\begin{array}{l}\text { Haakstad et al., } \\
2011^{102} \\
\text { Regular exercise } \\
\text { for all BMI with } \\
\text { sedentary } \\
\text { lifestyle }\end{array}$ & $\begin{array}{l}\text { Oslo, Norway } \\
\text { September } \\
2007 \text { - March } \\
2008\end{array}$ & $\begin{array}{l}105 \text { randomised } \\
52 \text { intervention group } \\
53 \text { control group } \\
\text { GDM: NR } \\
\text { age: } 31.2 \text { vs } 30.3 \\
\text { race: unclear } \\
\text { education: } \\
\text { college/university } \\
\text { education } 85 \% \text { vs } 85 \% \\
\text { SES: NR } \\
\text { PA: sedentary } \\
\text { occupation: } 71 \% \text { vs } \\
68 \% \\
\text { diet: NR } \\
\text { parity: NR } \\
\text { pre pregn weight: } 67.9\end{array}$ & $\begin{array}{l}\text { GWG: } \\
\text { difference } \\
\text { between self- } \\
\text { reported pre } \\
\text { pregnancy } \\
\text { weight and } \\
\text { weight at } \\
\text { completion of } \\
\text { intervention } \\
\text { period (36.6 } \\
\text { wks sd 0.95) } \\
\text { BMI: pre } \\
\text { pregnancy } \\
\text { weight and } \\
\text { unclear height } \\
\text { guidelines: }\end{array}$ & $\begin{array}{l}\text { 1. }<2 \text { times a week } 60 \text { min, } \\
\text { aerobic dance exercises, } \\
\text { strength training, stretching, } \\
\text { relaxation and body } \\
\text { awareness; led by certified } \\
\text { instructors; writing diary; } \\
\text { number of women in group } \\
\text { NR } \\
\text { 2. women were asked not to } \\
\text { change their PA pattern }\end{array}$ & $\begin{array}{l}\text { eligibility criteria specified }+ \\
\text { randomisation }+ \\
\text { allocation concealed + } \\
\text { baseline similar + } \\
\text { blinding of subjects - } \\
\text { blinding of therapists - } \\
\text { blinding of assessors + } \\
\text { at least } 85 \% \text { - } \\
\text { intention to treat analyses + } \\
\text { reporting with statistical } \\
\text { comparison + } \\
\text { point measures AND measures } \\
\text { of variability - } \\
\text { PEDro Sum score } 7\end{array}$ & $\begin{array}{l}\text { randomisation computer } \\
\text { generated } \\
\text { allocation: independent } \\
\text { person } \\
\text { personnel: } \\
\text { assessors: blinded } \\
\text { adherence: } 105 / 105= \\
100 \%\end{array}$ \\
\hline
\end{tabular}




\begin{tabular}{|c|c|c|c|c|c|c|}
\hline & & $\begin{array}{l}\text { vs } 68.4 \\
\text { weight: } 71.8 \text { vs } 72.7 \\
\text { height: } 169 \text { vs } 169 \\
\text { BMI: } 23.8 \text { vs } 23.9 \\
\mathrm{~kg} / \mathrm{m} 2 \\
\text { gest age at start: } 17.3 \\
\text { vs } 18.0\end{array}$ & $\begin{array}{l}\text { IOM 1990; OB } \\
5.0-9.0 \\
\text { UW: }<18.5 \\
\text { NW: } 18.5- \\
24.9 \\
\text { OW: } 25.0- \\
29.9 \\
\text { OB: }>30\end{array}$ & & & \\
\hline $\begin{array}{l}\text { Hui et al., } 2006 \\
93 \\
\text { Community } \\
\text { based exercise } \\
\text { and dietary } \\
\text { intervention for } \\
\text { all BMI }\end{array}$ & $\begin{array}{l}\text { Wiinipeg, } \\
\text { Canada } \\
\text { August } 2004 \text { - } \\
\text { April } 2005\end{array}$ & $\begin{array}{l}52 \text { randomised } \\
24 \text { intervention group } \\
21 \text { control group } \\
\text { GDM: NR } \\
\text { age: } 26.2 \text { vs } 26.2 \text { yr } \\
\text { race: aboriginal } 57 \% \text { vs } \\
38 \% \\
\text { education: } 11.8 \text { vs } \\
13.4 \text { yrs } \\
\text { SES: <30.000 \$: } 33 \% \text { vs } \\
29 \% \\
\text { PA: } 1.17 \text { vs } 1.52 \\
\text { diet: NR } \\
\text { parity: NR } \\
\text { pre pregn weight: NR } \\
\text { weight: NR } \\
\text { height: NR } \\
\text { BMI: } 25.7 \text { vs } 23.4 \\
\text { kg/m2 } \\
\text { gest age at start: NR }\end{array}$ & $\begin{array}{l}\text { GWG: NR } \\
\text { BMI: NR }\end{array}$ & $\begin{array}{l}\text { 1. Weekly instruction in group } \\
\text { session exercises and in home- } \\
\text { based exercise; computer } \\
\text { assisted Food Choice Map } \\
\text { dietary interviews and } \\
\text { counselling } \\
\text { 2. information package on diet } \\
\text { and activity for a healthy } \\
\text { pregnancy }\end{array}$ & $\begin{array}{l}\text { eligibility criteria specified + } \\
\text { randomisation - } \\
\text { allocation concealed - } \\
\text { baseline similar + } \\
\text { blinding of subjects - } \\
\text { blinding of therapists - } \\
\text { blinding of assessors - } \\
\text { at least } 85 \%+ \\
\text { intention to treat analyses + } \\
\text { reporting with statistical } \\
\text { comparison + } \\
\text { point measures AND measures } \\
\text { of variability - } \\
\text { PEDro Sum score } 5\end{array}$ & $\begin{array}{l}\text { randomisation: NR } \\
\text { allocation: NR } \\
\text { personnel: NR } \\
\text { assessors: NR } \\
\text { adherence: } 45 / 52=87 \%\end{array}$ \\
\hline $\begin{array}{l}\text { Ilmonen et al., } \\
2010^{94} \\
\text { probiotic with } \\
\text { diet vs placebo } \\
\text { and diet vs } \\
\text { placebo without } \\
\text { diet for all pre } \\
\text { BMI }\end{array}$ & $\begin{array}{l}\text { Turku, Finland } \\
\text { April } 2002- \\
\text { November } \\
2005\end{array}$ & $\begin{array}{l}256 \text { randomised } \\
85 \text { intervention } 1 \\
\text { group } \\
85 \text { intervention } 2 \\
\text { group } \\
85 \text { control Group } \\
\text { GDM: NR } \\
\text { age } 29.7 \text { vs } 30.1 \text { vs } \\
30.2 \text { yrs } \\
\end{array}$ & $\begin{array}{l}\text { GWG: } \\
\text { difference } \\
\text { between } \\
\text { weight within } \\
\text { one week } \\
\text { before delivery } \\
\text { minus self } \\
\text { reported pre } \\
\text { pregnancy }\end{array}$ & $\begin{array}{l}\text { 1. probiotics and dietary } \\
\text { intervention } \\
\text { 2. placebo and dietary } \\
\text { intervention } \\
\text { 3, placebo and no diet } \\
\text { dietary intervention: } 55-60 \% \\
\text { carbohydrates, } 30 \% \text { fat, } 10- \\
15 \% \text { protein, support, }\end{array}$ & $\begin{array}{l}\text { eligibility criteria specified + } \\
\text { randomisation + } \\
\text { allocation concealed + } \\
\text { baseline similar - } \\
\text { blinding of subjects + } \\
\text { blinding of therapists + } \\
\text { blinding of assessors + } \\
\text { at least } 85 \% \text { - } \\
\text { intention to treat analyses + }\end{array}$ & $\begin{array}{l}\text { randomisation computer } \\
\text { generated Block } \\
\text { allocation with sealed } \\
\text { envelops } \\
\text { personnel blinded } \\
\text { participants blinded } \\
\text { assessors blinded } \\
\text { adherence: unclear } \\
\text { concerning GWG 185/256 }\end{array}$ \\
\hline
\end{tabular}




\begin{tabular}{|c|c|c|c|c|c|c|}
\hline & & $\begin{array}{l}\text { race: NR } \\
\text { education: college or } \\
\text { university: } 79 \% \text { vs } 69 \% \\
\text { vs } 79 \% \\
\text { SES: NR } \\
\text { PA: NR } \\
\text { diet: NR } \\
\text { parity: first pregnancy: } \\
65 \% \text { vs } 51 \% \text { vs } 56 \% \\
\text { pre pregn weight: NR } \\
\text { weight at start: } 64.7 \text { vs } \\
71.0 \text { vs } 68.5 \text { p = } 0.004 \\
\text { height: NR } \\
\text { BMI: NR } \\
\text { gest age at start: NR }\end{array}$ & $\begin{array}{l}\text { weight } \\
\text { BMI: weight } \\
\text { and height } \\
\text { measured at } \\
\text { first visit } \\
\text { guidelines: } \\
\text { IOM } 1990 \text { (not } \\
\text { corresponding } \\
\text { with BMI- } \\
\text { categories in } \\
\text { this study) } \\
\text { NW: }<25.0 \\
\text { OW: } 25.0- \\
30.0 \\
\text { OB: }>30.0\end{array}$ & providing food & $\begin{array}{l}\text { reporting with statistical } \\
\text { comparison + } \\
\text { point measures AND measures } \\
\text { of variability + } \\
\text { PEDro Sum score } 9\end{array}$ & $=72 \%$ \\
\hline $\begin{array}{l}\text { Jackson et al., } \\
2011^{84} \\
\text { Diet, exercise } \\
\text { and video } \\
\text { counselling for } \\
\text { all BMI }\end{array}$ & $\begin{array}{l}\text { public } \\
\text { hospitals, } 5 \\
\text { prenatal care } \\
\text { practices and } \\
2 \text { academic } \\
\text { practices in } \\
\text { San Francisco } \\
\text { Bay Area, USA } \\
\text { June 2006 - } \\
\text { December } \\
2007\end{array}$ & $\begin{array}{l}327 \text { randomised } \\
163 \text { intervention group } \\
164 \text { control group } \\
\text { GDM: NR } \\
\text { age: } 26.1 \text { vs } 26.9 \text { yr } \\
\text { race: Hispanic } 39 \% \text { vs } \\
42 \% \text {; African-American } \\
24 \% \text { vs } 23 \% \text {; Asian } 16 \% \\
\text { vs } 13 \% \text {; White } 12 \% \text { vs } \\
13 \% \text {; other } 9 \% \text { vs } 8 \% \\
\text { education: < high } \\
\text { school } 20 \% \text { vs } 28 \% ; \\
\text { high school } 28 \% \text { vs } \\
17 \% \text {; > high school } \\
52 \% \text { vs } 55 \% \\
\text { SES: Medicaid } 85 \% \text { vs } \\
85 \% \\
\text { PA: sufficient exercise } \\
24 \% \text { vs } 20 \% \\
\text { diet: detailed in article } \\
\text { parity: first pregnancy: } \\
53 \% \text { vs } 46 \%\end{array}$ & $\begin{array}{l}\text { GWG: delivery } \\
\text { weight minus } \\
\text { self reported } \\
\text { pre pregnancy } \\
\text { weight } \\
\text { BMI: self } \\
\text { reported } \\
\text { weight and NR } \\
\text { height } \\
\text { guidelines: } \\
\text { IOM } 1990\end{array}$ & $\begin{array}{l}\text { 1. "Keep Fit" After baseline } \\
\text { assessment dietary } \\
\text { counselling focused on } \\
\text { increasing intake of fruits, } \\
\text { vegetables and whole grains, } \\
\text { increasing healthful fats and } \\
\text { decreasing sugary foods, } \\
\text { Video Doctor emphasized } \\
\text { tailored dietary and exercise } \\
\text { behaviour change over weight } \\
\text { gain; computer generated } \\
\text { worksheets for professional } \\
\text { and participant; }>4 \text { weeks } \\
\text { after a booster Video Doctor } \\
\text { counselling } \\
\text { 2. baseline assessment } \\
\text { without Video Doctor }\end{array}$ & $\begin{array}{l}\text { eligibility criteria specified + } \\
\text { randomisation + } \\
\text { allocation concealed + } \\
\text { baseline similar + } \\
\text { blinding of subjects - } \\
\text { blinding of therapists - } \\
\text { blinding of assessors + } \\
\text { at least } 85 \%+ \\
\text { intention to treat analyses + } \\
\text { reporting with statistical } \\
\text { comparison + } \\
\text { point measures AND measures } \\
\text { of variability - } \\
\text { PEDro Sum score } 8\end{array}$ & $\begin{array}{l}\text { randomisation computer } \\
\text { allocation by computer } \\
\text { program } \\
\text { personnel: not blinded } \\
\text { assessors: computer } \\
\text { program } \\
\text { adherence: } 287 / 327=88 \%\end{array}$ \\
\hline
\end{tabular}




\begin{tabular}{|c|c|c|c|c|c|c|}
\hline & & $\begin{array}{l}\text { pre pregn weight: NR } \\
\text { weight: NR } \\
\text { height: NR } \\
\text { BMI: } 27.3 \text { vs } 26.7 \\
\mathrm{~kg} / \mathrm{m} 2 \\
\text { gest age at start: } 19.7 \\
\text { vs } 19.1 \text { wk }\end{array}$ & & & & \\
\hline $\begin{array}{l}\text { Jeffries et al., } \\
2009^{101} \\
\text { weighing and } \\
\text { monitoring } \\
\text { GWG for all BMI }\end{array}$ & $\begin{array}{l}\text { tertiary } \\
\text { obstetric } \\
\text { hospital } \\
\text { Melbourne, } \\
\text { Australia } \\
\text { July } 2007 \text { - } \\
\text { October } 2007\end{array}$ & $\begin{array}{l}286 \text { randomised } \\
148 \text { intervention group } \\
138 \text { control group } \\
\text { GDM: NR } \\
\text { age: NR } \\
\text { race: NR } \\
\text { education: NR } \\
\text { SES: NR } \\
\text { PA: NR } \\
\text { diet: NR } \\
\text { parity: NR } \\
\text { pre pregn weight: NR } \\
\text { weight: } 68 \text { vs } 68 \text { kg } \\
\text { height: NR } \\
\text { BMI: UW: } 4 \% \text { vs } 5 \% \\
\text { NW: } 60 \% \text { vs } 60 \% \\
\text { OW: } 16 \% \text { vs } 16 \% \\
\text { OB: } 20 \% \text { vs } 19 \% \\
\text { gest age at start: } 11.6 \\
\text { vs } 11.4 \text { wks }\end{array}$ & $\begin{array}{l}\text { GWG: NR } \\
\text { BMI: NR } \\
\text { guidelines: } \\
\text { IOM } 1990 \\
\text { UW: }<19.8 \\
\text { NW } 19.8-26.0 \\
\text { OW: } 26.0- \\
29.0 \\
\text { OB: }>29.0\end{array}$ & $\begin{array}{l}\text { 1. women were given an } \\
\text { optimal GWG chart, defined } \\
\text { by pre BMI and IOM 1990; } \\
\text { advice to weigh at } 16,20,24 \text {, } \\
28,30,32.34 \text { wks } \\
\text { 2. brief dietary history taken } \\
\text { by midwives, written } \\
\text { information on healthy eating }\end{array}$ & $\begin{array}{l}\text { eligibility criteria specified + } \\
\text { randomisation + } \\
\text { allocation concealed + } \\
\text { baseline similar + } \\
\text { blinding of subjects + } \\
\text { blinding of therapists - } \\
\text { blinding of assessors + } \\
\text { at least } 85 \% \text { - } \\
\text { intention to treat analyses + } \\
\text { reporting with statistical } \\
\text { comparison + } \\
\text { point measures AND measures } \\
\text { of variability - } \\
\text { PEDro Sum score } 8\end{array}$ & $\begin{array}{l}\text { randomisation with } \\
\text { computer generated } \\
\text { allocation with numbered } \\
\text { cards in opaque envelops } \\
\text { participants blinded for } \\
\text { purpose of study } \\
\text { personnel blinded } \\
\text { assessors blinded } \\
\text { adherence: } 236 / 286=83 \%\end{array}$ \\
\hline $\begin{array}{l}\text { Luoto et al., } \\
2011^{95} \\
\text { Awareness of } \\
\text { GWG, PA and } \\
\text { dietary } \\
\text { individual } \\
\text { counselling for } \\
\text { GDM }\end{array}$ & $\begin{array}{l}\text { primary } \\
\text { health care } \\
\text { centres } \\
\text { Pirkanmaa } \\
\text { region, } \\
\text { Finland } \\
1 \text { October } \\
2007-31 \\
\text { December } \\
2008\end{array}$ & $\begin{array}{l}14 \text { municipalities } \\
\text { randomised } \\
219 \text { intervention group } \\
180 \text { control group } \\
\text { GDM: } 100 \% \\
\text { age: } 29.5 \text { vs } 30.0 \\
\text { race: NR } \\
\text { education: basic or } \\
\text { secondary: } 33.8 \% \text { vs } \\
33.7 \% \\
\text { SES: NR }\end{array}$ & $\begin{array}{l}\text { GWG: last } \\
\text { measured } \\
\text { weight during } \\
\text { pregnancy } \\
\text { minus pre } \\
\text { pregnancy } \\
\text { weight } \\
\text { BMI: pre } \\
\text { pregnancy } \\
\text { weight and } \\
\text { height NR }\end{array}$ & $\begin{array}{l}\text { 1. recommendations of GWG } \\
\text { discussed, PA ( } 4 \text { times) and } \\
\text { dietary counselling ( } 3 \text { times) } \\
\text { session; possibility of monthly } \\
\text { thematic meetings on PA } \\
\text { including group exercise; diets } \\
\text { with } 25-30 \% \text { fat, }<10 \% \\
\text { saccharose, } 25-35 \text { g/day fiber; } \\
\text { counselling cards, follow-up } \\
\text { notebooks } \\
\text { 2. no counselling beyond usual }\end{array}$ & $\begin{array}{l}\text { eligibility criteria specified + } \\
\text { randomisation + } \\
\text { allocation concealed + } \\
\text { baseline similar + } \\
\text { blinding of subjects - } \\
\text { blinding of therapists - } \\
\text { blinding of assessors - } \\
\text { at least } 85 \%+ \\
\text { intention to treat analyses + } \\
\text { reporting with statistical } \\
\text { comparison + }\end{array}$ & $\begin{array}{l}\text { randomised cluster } \\
\text { computer of pairs } \\
\text { allocation: NR } \\
\text { personnel not blinded } \\
\text { assessors not blinded } \\
\text { adherence: municipalities } \\
\text { 14/14 = 100\%; participants } \\
399 / 442=90 \%\end{array}$ \\
\hline
\end{tabular}




\begin{tabular}{|c|c|c|c|c|c|c|}
\hline & & $\begin{array}{l}\text { PA: NR } \\
\text { diet: NR } \\
\text { parity: first pregnancy: } \\
47 \% \text { vs } 40.6 \% \\
\text { pre pregn weight: NR } \\
\text { weight: NR } \\
\text { height: NR } \\
\text { BMI: } 26.3 \text { vs } 26.4 \\
\text { gest age at start: NR }\end{array}$ & & $\begin{array}{l}\text { care; some dietary } \\
\text { counselling, follow-up of } \\
\text { GWG, little PA counselling }\end{array}$ & $\begin{array}{l}\text { point measures AND measures } \\
\text { of variability + } \\
\text { PEDro Sum score } 8\end{array}$ & \\
\hline $\begin{array}{l}\text { Phelan et al., } \\
2011^{86} \\
\text { Behavioural } \\
\text { intervention on } \\
\text { diet, walking, } \\
\text { GWG and self } \\
\text { management } \\
\text { for all BMI }\end{array}$ & $\begin{array}{l}\text { Providence, } \\
\text { Rhode Island, } \\
\text { Canada } \\
2006-2008\end{array}$ & $\begin{array}{l}401 \text { randomised } \\
200 \text { intervention group } \\
201 \text { control group } \\
\text { GDM: NR } \\
\text { age } 28.6 \text { vs } 28.8 \text { yrs } \\
\text { race: White } 68.7 \% \text { vs } \\
67.5 \% \text {; Latina and } \\
\text { Hispanic } 19.6 \% \text { vs } \\
19.6 \% \text {; African- } \\
\text { American } 7.1 \% \text { vs } \\
9.6 \% \text {; other } 4.6 \% \text { vs } \\
3.3 \% \\
\text { education: high school } \\
12.1 \% \text { vs } 16.2 \% ; \text { some } \\
\text { college } 25.2 \% \text { vs } \\
27.9 \% ; \text { college degree } \\
35.4 \% \text { vs } 31.5 \% ; \\
\text { graduate degree } 27.3 \% \\
\text { vs } 24.4 \% \\
\text { SES: unemployed } \\
15.9 \% \text { vs } 18.5 \% \\
\text { PA: NR } \\
\text { diet: NR } \\
\text { parity: first pregnancy } \\
76.3 \% \text { vs } 76.6 \% \\
\text { pre pregn weight: NR } \\
\text { weight: NR } \\
\text { height: NR } \\
\text { BMI: } 26.32 \text { vs } 26.48\end{array}$ & $\begin{array}{l}\text { GWG: } \\
\text { difference } \\
\text { between last } \\
\text { measured } \\
\text { weight and pre } \\
\text { pregnancy } \\
\text { weight } \\
\text { BMI: self } \\
\text { reported } \\
\text { weight and } \\
\text { height at last } \\
\text { menstrual } \\
\text { period } \\
\text { guidelines: } \\
\text { IOM 1990; OB } \\
\text { max 11.4 kg } \\
\text { NW: } 19.8 \text { - } \\
26.0 \\
\text { OB/OW: } 26.1 \text { - } \\
40.0\end{array}$ & $\begin{array}{l}\text { 1. Fit for Delivery one face-to- } \\
\text { face visit with interventionist } \\
\text { on appropriate GWG, healthy } \\
\text { eating, PA } 30 \text { min walking } \\
\text { most days of the week, } \\
\text { stimulating self management, } \\
\text { weekly mailed materials and } \\
\text { telephone based feedback; } \\
\text { OW and OB additional support } \\
\text { 2. standard nutritional } \\
\text { counselling by physicians, } \\
\text { nurses, nutritionists and } \\
\text { counsellors from the Women, } \\
\text { Infants and Children's state } \\
\text { program, weighing by nurses } \\
\text { each clinical visit, newsletters } \\
\text { at } 2 \text { months intervals during } \\
\text { pregn and pp with general } \\
\text { pregnancy related issues }\end{array}$ & $\begin{array}{l}\text { eligibility criteria specified }+ \\
\text { randomisation }+ \\
\text { allocation concealed + } \\
\text { baseline similar + } \\
\text { blinding of subjects - } \\
\text { blinding of therapists - } \\
\text { blinding of assessors - } \\
\text { at least } 85 \%+ \\
\text { intention to treat analyses + } \\
\text { reporting with statistical } \\
\text { comparison + } \\
\text { point measures AND measures } \\
\text { of variability - } \\
\text { PEDro Sum score } 7\end{array}$ & $\begin{array}{l}\text { randomisation block } \\
\text { computer generated } \\
\text { allocation opaque } \\
\text { envelops } \\
\text { personnel not blinded } \\
\text { assessors NR } \\
\text { adherence: } 358 / 401=89 \%\end{array}$ \\
\hline
\end{tabular}




\begin{tabular}{|c|c|c|c|c|c|c|}
\hline & & $\begin{array}{l}\mathrm{kg} / \mathrm{m} 2 \\
\text { gest age at start: } 13.6 \\
\text { vs } 13.5 \text { wks }\end{array}$ & & & & \\
\hline $\begin{array}{l}\text { Polley et al., } \\
2002^{96} \\
\text { Behavioural } \\
\text { intervention on } \\
\text { diet, walking, } \\
\text { GWG and self } \\
\text { management } \\
\text { for NW and OW }\end{array}$ & $\begin{array}{l}\text { Pittsburgh } \\
\text { USA } \\
\text { time of } \\
\text { enrolment NR }\end{array}$ & $\begin{array}{l}120 \text { randomised } \\
61 \text { intervention group } \\
59 \text { control group } \\
\text { GDM: NR } \\
\text { age: mean } 25.5 \text { yrs } \\
\text { race: White: } 61 \% \text {; } \\
\text { Black } 39 \% \\
\text { education: high school } \\
\text { or less: } 45 \% \text {; some } \\
\text { college or vocational } \\
\text { training: } 42 \% \text {; college } \\
\text { graduate or graduate } \\
\text { training: } 13 \% \\
\text { SES: unemployment: } \\
57 \% \\
\text { PA: NR } \\
\text { diet: NR } \\
\text { parity: first pregnancy: } \\
47 \% \\
\text { pre pregn weight: NW } \\
62.1 \text { vs } 59 ; \text { OW } 83.6 \text { vs } \\
91.8 \text { kg } \\
\text { weight: NR } \\
\text { height: NR } \\
\text { BMI: NW } 22.8 \text { vs } 22.5 ; \\
\text { OW } 31.4 \text { vs } 34.1 \text { kg/m2 } \\
\text { gest age at start: NR }\end{array}$ & $\begin{array}{l}\text { GWG: last } \\
\text { weight at clinic } \\
\text { minus self } \\
\text { reported pre } \\
\text { pregnancy } \\
\text { weight at last } \\
\text { menstrual } \\
\text { period } \\
\text { BMI: NR } \\
\text { guidelines: } \\
\text { IOM 1990; OW } \\
+ \text { OB } 6.8-11.3 \\
\text { kg } \\
\text { NW: } 19.8- \\
26.0 \\
\text { OW > } 26.1\end{array}$ & $\begin{array}{l}\text { 1. one face-to-face visit on } \\
\text { appropriate GWG, healthy } \\
\text { eating, PA } 30 \text { min walking } \\
\text { most days of the week, } \\
\text { stimulating self management, } \\
\text { weekly mailed materials and } \\
\text { telephone based feedback; } \\
\text { OW and OB additional support } \\
\text { 2. standard nutritional } \\
\text { counselling, weighing each } \\
\text { clinical visit, newsletters at } 2 \\
\text { months intervals during pregn } \\
\text { and pp with general } \\
\text { pregnancy related issues }\end{array}$ & $\begin{array}{l}\text { eligibility criteria specified + } \\
\text { randomisation - } \\
\text { allocation concealed - } \\
\text { baseline similar - } \\
\text { blinding of subjects - } \\
\text { blinding of therapists - } \\
\text { blinding of assessors - } \\
\text { at least } 85 \%+ \\
\text { intention to treat analyses + } \\
\text { reporting with statistical } \\
\text { comparison + } \\
\text { point measures AND measures } \\
\text { of variability - } \\
\text { PEDro Sum score } 4\end{array}$ & $\begin{array}{l}\text { randomisation: "randomly } \\
\text { assigned" } \\
\text { allocation: NR } \\
\text { personnel: NR } \\
\text { assessors: NR } \\
\text { adherence: } 111 / 120=93 \% \\
\text { OW intervention group } \\
\text { lower weight at baseline } \\
\text { than control group }\end{array}$ \\
\hline $\begin{array}{l}\text { Quinlivan et al., } \\
2011^{87} \\
\text { Four step } \\
\text { multidisciplinary } \\
\text { approach for } \\
\text { OW and OB }\end{array}$ & $\begin{array}{l}\text { socio } \\
\text { economically } \\
\text { disadvantaged } \\
\text { area of } \\
\text { Melbourne, } \\
\text { Australia } \\
\text { time of } \\
\text { enrolment NR }\end{array}$ & $\begin{array}{l}132 \text { randomised } \\
67 \text { intervention group } \\
65 \text { control group } \\
\text { GDM: NR } \\
\text { age: } 28.3 \text { vs } 29.5 \\
\text { race: Caucasian: } 79 \% \\
\text { vs } 67 \% \text {; Asian: } 13 \% \text { vs } \\
26 \% \text {; other: } 8 \% \text { vs } 7 \%\end{array}$ & $\begin{array}{l}\text { GWG: } \\
\text { difference } \\
\text { between } \\
\text { booking weight } \\
\text { and weight at } \\
\text { onset of labour } \\
\text { BMI: NR }\end{array}$ & $\begin{array}{l}\text { 1. Four step multidisciplinary } \\
\text { approach; 1) continuity of care } \\
\text { provider, 2) weighing, 3) } \\
\text { dietary intervention by food } \\
\text { technologist at every } \\
\text { antenatal visit 4) psychological } \\
\text { assessment and intervention if } \\
\text { indicated }\end{array}$ & $\begin{array}{l}\text { eligibility criteria specified }+ \\
\text { randomisation }+ \\
\text { allocation concealed }+ \\
\text { baseline similar }+ \\
\text { blinding of subject - } \\
\text { blinding of therapists - } \\
\text { blinding of assessors + } \\
\text { at least } 85 \%+\end{array}$ & $\begin{array}{l}\text { randomisation computer } \\
\text { generated } \\
\text { allocation: opaque } \\
\text { envelops } \\
\text { personnel: NR } \\
\text { assessors: blinded } \\
\text { adherence } 124 / 132=94 \%\end{array}$ \\
\hline
\end{tabular}




\begin{tabular}{|c|c|c|c|c|c|c|}
\hline & & $\begin{array}{l}\text { education: NR } \\
\text { SES: NR } \\
\text { PA: NR } \\
\text { diet: NR } \\
\text { parity: first pregnancy } \\
32 \% \text { vs } 21 \% \\
\text { pre pregn weight: NR } \\
\text { weight: NR } \\
\text { height: NR } \\
\text { BMI: OW 42\% vs 49\%; } \\
\text { OB 58\% vs 51\% } \\
\text { gest age at start: NR }\end{array}$ & $\begin{array}{l}\text { OW: } 25-29.9 \\
\text { OB: > } 29.9\end{array}$ & $\begin{array}{l}\text { 2. routine public antenatal } \\
\text { care: midwifery, obstetrician } \\
\text { and PG antenatal clinics, with } \\
\text { access to high risk antenatal } \\
\text { clinics if necessary }\end{array}$ & $\begin{array}{l}\text { intention to treat analyses + } \\
\text { reporting with statistical } \\
\text { comparison + } \\
\text { point measures AND measures } \\
\text { of variability + } \\
\text { PEDro Sum score } 9\end{array}$ & \\
\hline $\begin{array}{l}\text { Rhodes et al., } \\
2010^{97} \\
\text { low fat versus } \\
\text { low glycaemic } \\
\text { load diet for } \\
\text { OW and OB }\end{array}$ & $\begin{array}{l}\text { Boston, USA } \\
\text { January } 2007 \\
\text { - June } 2009\end{array}$ & $\begin{array}{l}46 \text { randomised } \\
21 \text { low fat diet } \\
25 \text { low glycaemic load } \\
\text { diet } \\
\text { GDM: NR } \\
\text { age: } 33.2 \text { vs } 33.7 \\
\text { race: White: } 52 \% \text { vs } \\
56 \% \text {; Hispanic: } 14 \% \text { vs } \\
20 \% \text {; Black } 10 \% \text { vs } \\
12 \% \text {; other } 24 \% \text { vs } 12 \% \\
\text { education: BA or } \\
\text { higher } 76 \% \text { vs } 72 \% \\
\text { SES: NR } \\
\text { PA: NR } \\
\text { diet: NR } \\
\text { parity: NR } \\
\text { pre pregn weight: NR } \\
\text { weight: } 82.6 \text { vs } 88.4 \text { kg } \\
\text { BMI: } 31.2 \text { vs } 32.1 \\
\text { kg/m2 } \\
\text { gest age at start: } 19.6 \\
\text { vs } 19.8 \text { wks }\end{array}$ & $\begin{array}{l}\text { BMI: First } \\
\text { trimester or if } \\
\text { unavailable self } \\
\text { reported pre } \\
\text { pregnancy } \\
\text { weight and NR } \\
\text { height } \\
\text { GWG: NR }\end{array}$ & $\begin{array}{l}\text { 1. Low fat diet: } 25 \% \text { fat, } 55 \% \\
\text { carbohydrates, } 20 \% \text { protein } \\
\text { 2. Low glycaemic load diet: } \\
45 \% \text { carbohydrates, } 35 \% \text { fat, } \\
20 \% \text { protein } \\
\text { diets two } 1 \mathrm{hr} \text { in person } \\
\text { counselling session with } \\
\text { patient centred counselling } \\
\text { approach; written guides to } \\
\text { ensure consistency in delivery } \\
\text { of intervention messages; } \\
\text { providing bars and products }\end{array}$ & $\begin{array}{l}\text { eligibility criteria specified + } \\
\text { randomisation - } \\
\text { allocation concealed + } \\
\text { baseline similar + } \\
\text { blinding of subjects - } \\
\text { blinding of therapists + } \\
\text { blinding of assessors + } \\
\text { at least } 85 \% \text { - } \\
\text { intention to treat analyses + } \\
\text { reporting with statistical } \\
\text { comparison + } \\
\text { point measures AND measures } \\
\text { of variability + } \\
\text { PEDro Sumscore } 8\end{array}$ & $\begin{array}{l}\text { randomisation: "randomly } \\
\text { assigned" } \\
\text { allocation assignment } \\
\text { envelopes } \\
\text { personnel blinded } \\
\text { assessors blinded } \\
\text { adherence: } 38 / 46=83 \%\end{array}$ \\
\hline $\begin{array}{l}\text { Rosen et al., } \\
2003^{98} \\
\text { Nerve }\end{array}$ & $\begin{array}{l}\text { Morristown, } \\
\text { New Jersey; } \\
\text { Norfolk, } \\
\text { Virginia; }\end{array}$ & $\begin{array}{l}230 \text { randomised } \\
95 \text { intervention group } \\
92 \text { control group } \\
\text { GDM: NR }\end{array}$ & $\begin{array}{l}\text { WG: Difference } \\
\text { between end } \\
\text { and start of the } \\
\text { study }\end{array}$ & $\begin{array}{l}\text { 1. Nerve stimulation therapy } \\
\text { with Relief Band model on P6 } \\
\text { acupuncture point } \\
\text { 2. Same band without nerve }\end{array}$ & $\begin{array}{l}\text { eligibility criteria specified }+ \\
\text { randomisation }+ \\
\text { allocation concealed }+ \\
\text { baseline similar }+\end{array}$ & $\begin{array}{l}\text { randomisation computer } \\
\text { placebo controlled } \\
\text { allocation opaque } \\
\text { envelops }\end{array}$ \\
\hline
\end{tabular}




\begin{tabular}{|c|c|c|c|c|c|c|}
\hline $\begin{array}{l}\text { stimulation for } \\
\text { relief of nausea } \\
\text { and vomiting in } \\
\text { early pregnancy }\end{array}$ & $\begin{array}{l}\text { Tuscon, } \\
\text { Arizona; New } \\
\text { York, New } \\
\text { York, USA }\end{array}$ & $\begin{array}{l}\text { age: } 29.7 \text { vs } 29.3 \\
\text { race: White: } 76 \% \text { vs } \\
73 \% \text {; Black: } 8 \% \text { vs } 13 \% \text {; } \\
\text { Hispanic: } 12 \% \text { vs } 10 \% \text {; } \\
\text { Other } 4 \% \text { vs } 4 \% \\
\text { education: NR } \\
\text { SES: NR } \\
\text { PA: NR } \\
\text { diet: NR } \\
\text { parity: NR } \\
\text { pre pregn weight: NR } \\
\text { weight: } 67.3 \text { vs } 70.8 \mathrm{~kg} \\
\text { height: NR } \\
\text { BMI: } 25.0 \text { vs } 25.8 \\
\text { kg/m2 } \\
\text { gest age at start: } 9.2 \\
\text { vs } 9.0 \\
\text { nausea or vomiting }\end{array}$ & BMI: NR & stimulation & $\begin{array}{l}\text { blinding of subjects + } \\
\text { blinding of therapists - } \\
\text { blinding of assessors - } \\
\text { at least } 85 \% \text { - } \\
\text { intention to treat analyses + } \\
\text { reporting with statistical } \\
\text { comparison + } \\
\text { point measures AND measures } \\
\text { of variability - } \\
\text { PEDro Sum score } 7\end{array}$ & $\begin{array}{l}\text { personnel not blinded } \\
\text { assessors not blinded } \\
\text { adherence: } 187 / 230=81 \%\end{array}$ \\
\hline $\begin{array}{l}\text { Thornton } 2009 \\
103 \\
\text { diet with } \\
\text { support for OB }\end{array}$ & $\begin{array}{l}\text { tertiary care } \\
\text { medical } \\
\text { centres } \\
\text { USA } \\
\text { June } 1998 \text { - } \\
\text { May } 2005\end{array}$ & $\begin{array}{l}257 \text { randomised } \\
124 \text { intervention group } \\
133 \text { control group } \\
\text { GDM: NR } \\
\text { age: } 27.3 \text { vs } 26.8 \\
\text { race: African American } \\
42.2 \% \text { vs } 39.7 \% \text {; } \\
\text { Caucasian } 23.3 \% \text { vs } \\
21.6 \% \text {; Latina } 21.6 \% \text { vs } \\
25 \% \text { Indian } 12.9 \% \text { vs } \\
13.7 \% \\
\text { education: NR } \\
\text { SES: NR } \\
\text { PA: NR } \\
\text { diet: NR } \\
\text { parity: nulliparous: } \\
17.2 \% \text { vs } 16.3 \% \\
\text { pre pregn weight: NR } \\
\text { weight: } 97.2 \text { vs } 92.6\end{array}$ & $\begin{array}{l}\text { GWG: } \\
\text { difference } \\
\text { between } \\
\text { baseline weight } \\
\text { and last weight } \\
\text { before delivery } \\
\text { BMI: self } \\
\text { reported pre } \\
\text { pregnancy } \\
\text { weight and } \\
\text { height }\end{array}$ & $\begin{array}{l}\text { 1. detailed dietary intake } \\
\text { protocol by registered } \\
\text { dietician; compared to } \\
\text { protocol for GDM: } 18-24 \\
\text { kacal/kg balanced, } 40 \% \\
\text { carbohydrates, } 30 \% \text { protein, } \\
30 \% \text { fat; at least } 2000 \text { kcal, } \\
\text { Diary record; physician } \\
\text { reviewed these diaries each } \\
\text { visit } \\
\text { 2. basic dietary advice by } \\
\text { registered dietician } \\
\text { both groups weighing at each } \\
\text { prenatal visit; both groups } \\
\text { advised to walk } 30 \text { minutes } \\
\text { per day }\end{array}$ & $\begin{array}{l}\text { eligibility criteria specified }+ \\
\text { randomisation }+ \\
\text { allocation concealed + } \\
\text { baseline similar }+ \\
\text { blinding of subjects - } \\
\text { blinding of therapists - } \\
\text { blinding of assessors - } \\
\text { at least } 85 \% \text { - } \\
\text { intention to treat analyses + } \\
\text { reporting with statistical } \\
\text { comparison + } \\
\text { point measures AND measures } \\
\text { of variability + } \\
\text { PEDro Sum score } 7\end{array}$ & $\begin{array}{l}\text { randomisation Block with } \\
\text { random number table } \\
\text { allocation: sealed } \\
\text { envelops } \\
\text { personnel: not blinded } \\
\text { assessor: NR } \\
\text { adherence: } 232 / 257=83 \%\end{array}$ \\
\hline
\end{tabular}




\begin{tabular}{|c|c|c|c|c|c|c|}
\hline & & $\begin{array}{l}\text { height: NR } \\
\text { BMI: } 38.22 \text { vs } 37.41 \\
\text { kg/m2 } \\
\text { gest age at start: NR }\end{array}$ & & & & \\
\hline $\begin{array}{l}\text { Wolff et al., } \\
2008^{99} \\
\text { diet with } \\
\text { support for OB }\end{array}$ & $\begin{array}{l}\text { Frederiksberg } \\
\text { and } \\
\text { Copenhagen, } \\
\text { Den-mark } \\
\text { Period of } \\
\text { recruitment } \\
\text { NR }\end{array}$ & $\begin{array}{l}64 \text { randomised } \\
28 \text { intervention group } \\
38 \text { control group } \\
\text { GDM: NR } \\
\text { mean age: } 28.4 \text { vs } 30 \\
\text { race: Caucasian } \\
\text { education: NR } \\
\text { SES: NR } \\
\text { PA: NR } \\
\text { diet: NR } \\
\text { parity: nulliparous: } \\
17.2 \% \text { vs } 16.3 \% \\
\text { pre pregn weight: } 97.0 \\
\text { vs } 95.6 \\
\text { weight: } 97.5 \text { vs } 97.9 \\
\text { height: } 167.3 \text { vs } 167.9 \\
\text { BMI: } 34.9 \text { vs } 34.6 \\
\text { kg/m2 } \\
\text { gest age at start: } 15 \text { vs } \\
16 \text { wks }\end{array}$ & $\begin{array}{l}\text { GWG: } \\
\text { difference } \\
\text { between } \\
\text { weight just } \\
\text { before delivery } \\
\text { and self } \\
\text { reported pre } \\
\text { pregnancy } \\
\text { weight } \\
\text { rate of GWG: } \\
\text { difference } \\
\text { between } \\
\text { measured } \\
\text { weight at } \\
\text { inclusion and } \\
36 \text { weeks of } \\
\text { gestation } \\
\text { divided by } \\
\text { numbers of } \\
\text { weeks }\end{array}$ & $\begin{array}{l}\text { 1. } 10 \text { consultations of } 1 \mathrm{hr} \\
\text { with trained dietician to eat } \\
\text { according to Danish } \\
\text { recommendations (fat intake < } \\
30 \% \text {, protein } 15-20 \% \text {, } \\
\text { carbohydrates } 50-55 \% \text { ) total } \\
\text { energy intake individual } \\
\text { estimated: basic metabolic } \\
\text { rate x } 1.4 \text {; dietary } \\
\text { supplements } \\
\text { 2. no consults, no restrictions } \\
\text { on energy intake or GWG }\end{array}$ & $\begin{array}{l}\text { eligibility criteria specified + } \\
\text { randomisation }+ \\
\text { allocation concealed - } \\
\text { baseline similar + } \\
\text { blinding of subjects - } \\
\text { blinding of therapists + } \\
\text { blinding of assessors + } \\
\text { at least } 85 \% \text { - } \\
\text { intention to treat analyses + } \\
\text { reporting with statistical } \\
\text { comparison + } \\
\text { point measures AND measures } \\
\text { of variability - } \\
\text { PEDro Sum score } 7\end{array}$ & $\begin{array}{l}\text { randomisation } \\
\text { computerised } \\
\text { allocation } \\
\text { personnel: blinded } \\
\text { assessors: blinded } \\
\text { adherence: } 50 / 64=78 \%\end{array}$ \\
\hline
\end{tabular}

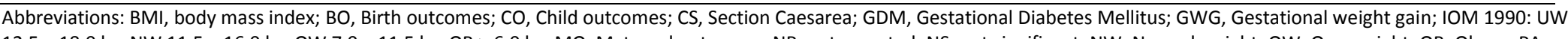
$12.5-18.0$ kg, NW $11.5-16.0$ kg, OW $7.0-11.5$ kg, OB > 6.0 kg; MO, Maternal outcomes; NR, not reported; NS, not significant; NW, Normal weight; OW, Overweight; OB, Obese; PA, Physical Activity; PIH, pregnancy induced hypertension; SES, Social Economic Status; UW, Underweight; ... vs ..., intervention versus control as numbered in column about intervention 
Table 2.2 Quality assessment and characteristics non-Randomised Controlled Trials (RCT's)

\begin{tabular}{|c|c|c|c|c|c|c|}
\hline $\begin{array}{l}\text { Author, year } \\
\text { interventio } \\
\mathrm{n} \text { in short }\end{array}$ & Setting & $\begin{array}{l}\text { Characteristics of study- } \\
\text { population on baseline }\end{array}$ & Definitions & Comparison & Intervention & GRADE score \\
\hline $\begin{array}{l}\text { Artal et al. } \\
2007^{106} \\
\text { Lifestyle } \\
\text { intervention } \\
\text { for OB with } \\
\text { GDM }\end{array}$ & $\begin{array}{l}\text { St. Louis, USA } \\
\text { period of } \\
\text { recruitment NR }\end{array}$ & $\begin{array}{l}39 \text { women in intervention } \\
\text { group } \\
57 \text { women in control group } \\
\text { GDM: } 100 \% \\
\text { age: } 32.4 \text { vs } 30.6 \text { yrs } \\
\text { race: Caucasian: } 61.5 \% \text { vs } \\
56.1 \% \text {; African-American } \\
28.2 \% \text { vs } 29.8 \% \text {; other } 10.2 \% \\
\text { vs } 14.1 \% \\
\text { education: NR } \\
\text { SES: NR } \\
\text { PA: NR } \\
\text { diet: NR } \\
\text { parity: gravity: } 2.4 \text { vs } 2.7 \\
\text { pre pregn weight: NR } \\
\text { weight: NR } \\
\text { height: NR } \\
\text { BMI: > } 25 \mathrm{~kg} / \mathrm{m}^{2} \\
\text { gest age at start: } 29.4 \text { vs } 28.0 \\
\text { wks }\end{array}$ & $\begin{array}{l}\text { guidelines: NR } \\
\text { GWG: difference } \\
\text { between last } \\
\text { obstetric visit } \\
\text { and first visit } \\
\text { BMI: NR } \\
\text { weight gain } \\
\text { rate: GWG } \\
\text { divided by weeks } \\
\text { of pregnancy in } \\
\text { between }\end{array}$ & $\begin{array}{l}\text { subjects could choose } \\
\text { the intervention } \\
\text { exercise and diet or } \\
\text { diet; subjects who } \\
\text { declined were in the } \\
\text { diet group; } \\
\text { when insulin was } \\
\text { required exclusion }\end{array}$ & $\begin{array}{l}\text { 1. exercise } 60 \% \text { VO2max } \\
\text { walking or cycling once a } \\
\text { week with supervision } \\
\text { and encouraging to do } \\
\text { that every day, normal } \\
\text { care } \\
\text { 2. no exercise, normal } \\
\text { care } \\
\text { normal care: education on } \\
\text { healthy low fat, } \\
\text { consistent carbohydrate } \\
\text { intake at meals; weight } \\
\text { gain goals according to } \\
\text { IOM } 1990 \text { by dietician }\end{array}$ & $\begin{array}{l}\text { Downgrading } \\
\text { limitations in design: + } \\
\text { indirectness of evidence: - } \\
\text { unexplained heterogeneity: - } \\
\text { imprecision of results: - } \\
\text { high probability publication bias: } \\
\text { - } \\
\text { Upgrading: } \\
\text { absence of confounders: - } \\
\text { effect likely larger then found: - } \\
\text { dose-response gradient present: } \\
\text { - } \\
\text { Total: very low }\end{array}$ \\
\hline $\begin{array}{l}\text { Claesson et } \\
\text { al. } 2008^{107} \\
\text { Weekly } \\
\text { motivational } \\
\text { talks and } \\
\text { aqua aerobic } \\
\text { classes for OB }\end{array}$ & $\begin{array}{l}\text { antenatal care } \\
\text { clinics Linköping } \\
\text { and two nearby } \\
\text { cities, } \\
\text { Sweden } \\
\text { recruiting } \\
\text { between } \\
\text { November } 2003 \\
\text { and December } \\
2005\end{array}$ & $\begin{array}{l}160 \text { women in intervention } \\
\text { group } \\
208 \text { women in control group } \\
\text { GDM: } 0 \% \\
\text { age: } 29 \text { vs } 30 \text { yrs (S) } \\
\text { race: NR } \\
\text { education: NR } \\
\text { SES: employed: } 64.5 \% \text { vs } \\
71.6 \% \\
\text { PA: NR } \\
\text { diet: NR } \\
\text { parity: nulliparous: } 41.9 \& \text { vs } \\
47.7 \%\end{array}$ & $\begin{array}{l}\text { guidelines: NR } \\
\text { GWG: weight at } \\
\text { last visit minus } \\
\text { weight at first } \\
\text { visit } \\
\text { BMI: NR }\end{array}$ & $\begin{array}{l}\text { subjects in Linköping } \\
\text { (rate of obesity } 8.4 \% \text { ) } \\
\text { got the intervention; } \\
\text { in the two nearby } \\
\text { cities (rate of obesity } \\
7.3 \% \text { ) subjects had no } \\
\text { intervention }\end{array}$ & $\begin{array}{l}\text { 1. extra visits with trained } \\
\text { midwife using sort of } \\
\text { Motivational } \\
\text { Interviewing; } 30 \text { min a } \\
\text { week, invited to aqua } \\
\text { aerobic } 1 \text { a } 2 \text { /wk for } \\
\text { obese women } \\
\text { 2. routine care antenatal } \\
\text { programme }\end{array}$ & $\begin{array}{l}\text { Downgrading } \\
\text { limitations in design: - } \\
\text { indirectness of evidence: - } \\
\text { unexplained heterogeneity: - } \\
\text { imprecision of results: - } \\
\text { high probability publication bias: } \\
\text { - } \\
\text { Upgrading: } \\
\text { absence of confounders: + } \\
\text { effect likely larger then found: - } \\
\text { dose-response gradient present: } \\
\text { - }\end{array}$ \\
\hline
\end{tabular}


height: NR

BMI: obese; BMI 30.0 - 34.9:

$64.5 \%$ vs $65.8 \%$; BMI $35.0-$

39.9: $23.2 \%$ vs $21.8 \%$; $\mathrm{BMI}>$

40: $12.3 \%$ vs $12.4 \%$

gest age at start: NR

\begin{tabular}{ll}
\hline $\begin{array}{l}\text { Garg et al. } \\
2006^{108}\end{array}$ & $\begin{array}{l}\text { Indragarhi, } \\
\text { Uttar Pradesh, } \\
\text { India }\end{array}$ \\
$\begin{array}{l}\text { Nutritional } \\
\text { counselling }\end{array}$ & $\begin{array}{l}\text { period of } \\
\text { for low SES }\end{array}$
\end{tabular}

50 intervention group

50 control group

GDM: NR

guidelines: NR
GWG: NR
BMI: NR

subjects were

selected by

domiciliary visits;

1. Identifying lacunes,

providing simple nutrition

education, weighing,

when they

home visits, group

age: $82 \%$ between $20-29 \mathrm{yr}$;

administered late in

race: NR

education: $57 \%$ illiterate; $95 \%$

pregnancy, they were

meetings, social

included in the

environment support,

control group;

reinforcement

housewife

SES: low; average income Rs

966.5 (= €10.17)

PA: NR

subject who

2. unclear

administered early in

pregnancy were

diet: much below

recommended guidelines

parity: nulliparous: $27 \%$

pre pregn weight: NR

weight: $52.75 \mathrm{~kg}$

height: $154.3 \mathrm{~cm}$

BMI: NR

included in

intervention group

\section{Downgrading}

limitations in design: +

indirectness of evidence: +

unexplained heterogeneity: -

imprecision of results: -

high probability publication bias:

(at start: early vs late

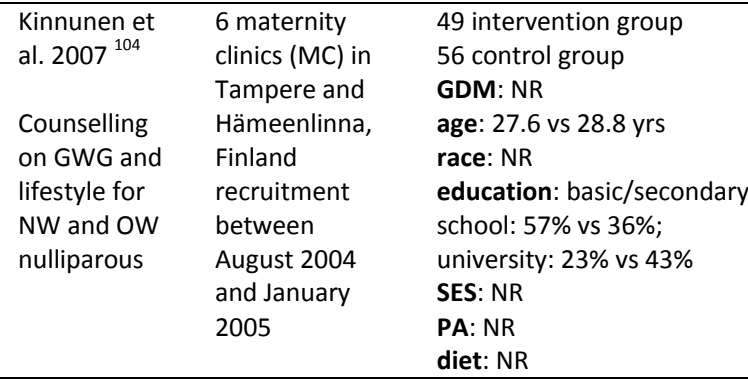

\section{guidelines: IOM}

1990

GWG: NR

BMI: pre

pregnancy

height and

weight

UW<20; NW 20-

26; $O W>26$ health nurses from 3

MC's volunteered to

do the intervention

for all their eligible

participants;

remaining $3 \mathrm{MC}^{\prime} \mathrm{s}$

were control clinic

diet: NR
1. information on

recommended GWG (UW

12.5-18 kg; NW 11.5-16.0

kg; OW 7.0-11.5 kg),

dietary counselling (4

times in pregnancy), PA

counselling ( 5 times in

pregnancy) above

standard car

2. standard care: $11-15$

visits to public health

\section{Upgrading:}

absence of confounders: -

effect likely larger then found: -

dose-response gradient present:

Total: very low

\section{Downgrading}

limitations in design: -

indirectness of evidence: -

unexplained heterogeneity: -

imprecision of results: -

high probability publication bias:

\section{Upgrading:}

absence of confounders: +

effect likely larger then found: + 


\begin{tabular}{|c|c|c|c|c|c|c|}
\hline & & $\begin{array}{l}\text { parity: nulliparous } 100 \% \\
\text { pre pregn weight: } 65.7 \text { vs } 61.0 \\
\text { kg } \\
\text { weight: NR } \\
\text { height: } 166.4 \text { vs } 166.6 \mathrm{~cm} \\
\text { BMI: } 23.7 \text { vs } 22.3 ; \text { UW: } 8 \% \text { vs } \\
18 \% \text {; NW: } 75 \% \text { vs } 79 \% ; \text { OW } \\
17 \% \text { vs } 4 \% \\
\text { gest age at start: NR }\end{array}$ & & & $\begin{array}{l}\text { nurse and } 3 \text { visits to } \\
\text { physician }\end{array}$ & $\begin{array}{l}\text { dose-response gradient present: } \\
\text { Total: high }\end{array}$ \\
\hline $\begin{array}{l}\text { Klima et al. } \\
2009109 \\
\text { Group visits } \\
\text { with } \\
\text { discussing } \\
\text { health issues } \\
\text { for low SES }\end{array}$ & $\begin{array}{l}\text { Midwest, USA } \\
\text { birth between } \\
\text { December } 2004 \\
\text { and October } \\
2006\end{array}$ & $\begin{array}{l}110 \text { intervention group } \\
207 \text { comparison group } \\
\text { GDM: NR } \\
\text { age: } 20.8 \text { vs } 22.1 \text { yrs mean } \\
21.8 \text { ( } 14-38) \text { yrs } \\
\text { race: African-American: } 98 \% \\
\text { education: NR } \\
\text { SES: Medicaid } 100 \% \\
\text { PA: NR } \\
\text { diet: NR } \\
\text { parity: NR } \\
\text { pre pregn weight: NR } \\
\text { weight: NR } \\
\text { height: NR } \\
\text { BMI: NR } \\
\text { gest age at start: < } 18 \text { wks }\end{array}$ & $\begin{array}{l}\text { guidelines: NR } \\
\text { GWG: NR } \\
\text { BMI: NR }\end{array}$ & $\begin{array}{l}\text { All women }<18 \text { wks } \\
\text { pregnant were } \\
\text { offered Centering } \\
\text { Pregnancy Care; } \\
\text { women who declined } \\
\text { or women who } \\
\text { were }>18 \text { wks } \\
\text { pregnant were in the } \\
\text { control group }\end{array}$ & $\begin{array}{l}\text { 1. Centering Pregnancy } \\
\text { Care: group visits with } \\
\text { discussing health issues } \\
\text { 2. Individualised care }\end{array}$ & $\begin{array}{l}\text { Downgrading } \\
\text { limitations in design: + } \\
\text { indirectness of evidence: - } \\
\text { unexplained heterogeneity: - } \\
\text { imprecision of results: - } \\
\text { high probability publication bias: } \\
\text { - } \\
\text { Upgrading: } \\
\text { absence of confounders: - } \\
\text { effect likely larger then found: - } \\
\text { dose-response gradient present: } \\
\text { - } \\
\text { Total: very low }\end{array}$ \\
\hline $\begin{array}{l}\text { Mc Donough } \\
2003^{110} \\
\text { Case manager } \\
\text { during } \\
\text { pregnancy for } \\
\text { low SES }\end{array}$ & $\begin{array}{l}\text { South Carolina, } \\
\text { USA } \\
\text { birth between } 1 \\
\text { January and } 31 \\
\text { December } 1995\end{array}$ & $\begin{array}{l}205 \text { nursing case managed } \\
\text { group } \\
831 \text { other case managed } \\
\text { group } \\
892 \text { No case managed group } \\
\text { GDM: NR } \\
\text { age: } 24.2 \text { vs } 21.4 \text { vs } 22.8 \text { yrs; } \\
\text { mean age } 22.3 \text { yrs } \\
\text { race: negroid: } 71.7 \% \text { vs } 71.1 \% \\
\text { vs } 63.8 \% \text {; Caucasian: } 27.3 \% \text { vs } \\
28.0 \% \text { vs } 35.1 \% \\
\text { education: mean years: } 11.5 \\
\text { vs } 11.6 \text { vs } 12.1\end{array}$ & $\begin{array}{l}\text { guidelines: NR } \\
\text { GWG: NR } \\
\text { BMI: NR }\end{array}$ & $\begin{array}{l}\text { retrospective study of } \\
\text { data }\end{array}$ & $\begin{array}{l}\text { 1. contacts with a case } \\
\text { manager which is a nurse } \\
\text { during pregnancy } \\
\text { 2. contacts with a case } \\
\text { manager with others } \\
\text { during pregnancy } \\
\text { 3. no case manager, } \\
\text { though normal antenatal } \\
\text { care visits }\end{array}$ & $\begin{array}{l}\text { Downgrading } \\
\text { limitations in design: - } \\
\text { indirectness of evidence: + } \\
\text { unexplained heterogeneity: - } \\
\text { imprecision of results: - } \\
\text { high probability publication bias: } \\
+ \\
\text { Upgrading: } \\
\text { absence of confounders: - } \\
\text { effect likely larger then found: - } \\
\text { dose-response gradient present: } \\
\text { - }\end{array}$ \\
\hline
\end{tabular}




\begin{tabular}{|c|c|c|c|c|c|c|}
\hline & & $\begin{array}{l}\text { SES: Medicaid } 100 \% \\
\text { PA: NR } \\
\text { diet: NR } \\
\text { parity: nulliparous } 31.7 \% \text { vs } \\
58.4 \% \text { vs } 42.7 \% \\
\text { pre pregn weight: NR } \\
\text { weight: NR } \\
\text { height: NR } \\
\text { BMI: NR } \\
\text { gest age at start: NR }\end{array}$ & & & & Total: very low \\
\hline $\begin{array}{l}\text { Mottola } 2010 \\
\text { I11 } \\
\text { Individual } \\
\text { lifestyle } \\
\text { intervention } \\
\text { plan for OW } \\
\text { and OB }\end{array}$ & $\begin{array}{l}\text { London, } \\
\text { Ontario, Canada } \\
\text { period of } \\
\text { recruitment NR }\end{array}$ & $\begin{array}{l}65 \text { intervention group } \\
260 \text { comparison group } \\
\text { GDM: NR } \\
\text { age: } 32.4 \text { vs } 31.9 \text { yrs } \\
\text { race: NR } \\
\text { education: NR } \\
\text { SES: NR } \\
\text { PA: NR } \\
\text { diet: NR } \\
\text { parity: previous pregnancy } \\
62 \% \text { vs } 62 \% \\
\text { pre pregn weight: } 88.3 \text { vs } 85.5 \\
\text { kg } \\
\text { weight: NR } \\
\text { height: } 165.9 \text { vs } 164.0 \mathrm{~cm} \\
\text { BMI: } 32.1 \text { vs } 33.4 \mathrm{~kg} / \mathrm{m} 2 ; \text { OW } \\
48 \% \text { vs } 48 \% \text {; OB } 52 \% \text { vs } 52 \% \\
\text { gest age at start: first } \\
\text { trimester }\end{array}$ & $\begin{array}{l}\text { guidelines: } \\
\text { EGWG = >10.6 } \\
\text { kg } \\
\text { GWG: difference } \\
\text { between last } \\
\text { visit and first } \\
\text { visit } \\
\text { BMI: self- } \\
\text { reported pre } \\
\text { pregnancy } \\
\text { weight and } \\
\text { height: OW: } 25.0 \\
\text { - 29.9; OB: > } 30\end{array}$ & $\begin{array}{l}\text { subjects were self- } \\
\text { applying by } \\
\text { recruitment from } \\
\text { health care providers } \\
\text { and advertisement; } \\
\text { matching (pBMI, } \\
\text { maternal age, parity) } \\
\text { with historical cohort } \\
\text { from large local } \\
\text { perinatal database in } \\
\text { the same time frame }\end{array}$ & $\begin{array}{l}\text { 1. Nutrition and Exercise } \\
\text { Lifestyle Intervention } \\
\text { Program; individualized } \\
\text { meal plan, feedback, } \\
\text { support, individualized } \\
\text { exercise program (mild to } \\
\text { moderated) } \\
\text { 2. usual care }\end{array}$ & $\begin{array}{l}\text { Downgrading } \\
\text { limitations in design: + } \\
\text { indirectness of evidence: - } \\
\text { unexplained heterogeneity: - } \\
\text { imprecision of results: - } \\
\text { high probability publication bias: } \\
\text { - } \\
\text { Upgrading: } \\
\text { absence of confounders: - } \\
\text { effect likely larger then found: - } \\
\text { dose-response gradient present: } \\
\text { - } \\
\text { Total: very low }\end{array}$ \\
\hline $\begin{array}{l}\text { Olson } 2004 \\
112 \\
\text { Guidance and } \\
\text { monitoring } \\
\text { weight and } \\
\text { education on } \\
\text { diet and PA } \\
\text { for NW and }\end{array}$ & $\begin{array}{l}\text { rural area } \\
\text { New York, USA } \\
\text { recruited from } \\
\text { March 2000 to } \\
\text { April 2001; } \\
\text { control group } \\
\text { recruited April } \\
1995 \text { to April } \\
1997\end{array}$ & $\begin{array}{l}197 \text { intervention group } \\
381 \text { comparison group } \\
\text { GDM: NR } \\
\text { age: }<20 \text { yrs: } 3.4 \% \text { vs } 3.4 \% ; \\
20-40 \text { yrs: } 93.9 \% \text { vs } 93.7 \% \\
\text { race: Caucasian: } 96 \% \\
\text { education: } \leq 12 \text { yrs: } 30.9 \% \text { vs } \\
37.0 \% \\
\text { SES: }>185 \% \text { poverty line } 62.6 \&\end{array}$ & $\begin{array}{l}\text { guidelines: IOM } \\
1990 \\
\text { GWG: last visit } \\
\text { weight minus } \\
\text { weight at first } \\
\text { visit; for women } \\
\text { who attended } \\
\text { later than } 12 \\
\text { wks pregnancy }\end{array}$ & $\begin{array}{l}\text { NW and OW women } \\
\text { were recruited and } \\
\text { compared with a } \\
\text { historical cohort from } \\
\text { another study in the } \\
\text { same area }\end{array}$ & $\begin{array}{l}\text { 1. monitoring weight gain } \\
\text { with guidance and by mail } \\
\text { patient education } \\
\text { program, focused on } \\
\text { appropriate weight gain, } \\
\text { healthy diet and exercise } \\
\text { 2. usual care during } \\
\text { observational study }\end{array}$ & $\begin{array}{l}\text { Downgrading } \\
\text { limitations in design: - } \\
\text { indirectness of evidence: + } \\
\text { unexplained heterogeneity: - } \\
\text { imprecision of results: - } \\
\text { high probability publication bias: } \\
\text { - } \\
\text { Upgrading: }\end{array}$ \\
\hline
\end{tabular}




\begin{tabular}{|c|c|c|c|c|c|c|}
\hline OW & & $\begin{array}{l}\text { vs } 56.7 \% \\
\text { PA: NR } \\
\text { diet: NR } \\
\text { parity: nulliparous: } 41.3 \% \text { vs } \\
41.1 \% \\
\text { pre pregn weight: NR } \\
\text { weight: NR } \\
\text { height: NR } \\
\text { BMI: NW: } 73.2 \% \text { vs } 76.1 \% \text {; } \\
\text { OW } 26.8 \% \text { vs } 23.9 \% \\
\text { gest age at start: NR }\end{array}$ & $\begin{array}{l}\text { weight was } \\
\text { adjusted } \\
\text { BMI: NW: } 19.8- \\
\text { 26.0; OW: } 26.1- \\
29.0\end{array}$ & & & $\begin{array}{l}\text { absence of confounders: - } \\
\text { effect likely larger then found: - } \\
\text { dose-response gradient present: } \\
+ \\
\text { Total: low }\end{array}$ \\
\hline $\begin{array}{l}\text { Shirazian } \\
2010^{105} \\
\text { Behavioural } \\
\text { lifestyle } \\
\text { program for } \\
\text { OB with low } \\
\text { SES }\end{array}$ & $\begin{array}{l}\text { New York city, } \\
\text { USA } \\
\text { recruited from } \\
2007 \text { to } 2008\end{array}$ & $\begin{array}{l}21 \text { intervention group } \\
20 \text { comparison group } \\
\text { GDM: NR } \\
\text { age: } 29.0 \text { vs } 24.4 \text { yrs S } \\
\text { race: Latina: } 67 \% \text { vs } 80 \% \text {; } \\
\text { Negroid } 33 \% \text { vs } 20 \% \\
\text { education: NR } \\
\text { SES: } 100 \% \text { Medicaid } \\
\text { PA: NR } \\
\text { diet: NR } \\
\text { parity: nulliparous: } 19 \% \text { vs } \\
\text { 50\% S } \\
\text { pre pregn weight: NR } \\
\text { weight: NR } \\
\text { height: NR } \\
\text { BMI: } 36.2 \text { vs } 34.24 \mathrm{~kg} / \mathrm{m} 2 \\
\text { gest age at start: NR }\end{array}$ & $\begin{array}{l}\text { guidelines: NR } \\
\text { GWG: NR } \\
\text { BMI: height and } \\
\text { weight } \\
\text { measured at first } \\
\text { visit }\end{array}$ & $\begin{array}{l}\text { subjects were } \\
\text { recruited by all health } \\
\text { care providers; not } \\
\text { clear how the } \\
\text { intervention group } \\
\text { and the control group } \\
\text { was assembled }\end{array}$ & $\begin{array}{l}\text { 1. behavioural } \\
\text { modification program; } \\
\text { materials to educate } \\
\text { women on obesity and } \\
\text { pregnancy, healthy } \\
\text { eating, possible dietary } \\
\text { improvements, calorie } \\
\text { counting, encourage } \\
\text { walking as exercise; food } \\
\text { diary, pedometer; } 6 \\
\text { seminars, one-to-one } \\
\text { counselling and phone } \\
\text { calls; repeating the goal } \\
\text { of maximum } 15 \text { pounds } \\
\text { GWG } \\
\text { 2. NR }\end{array}$ & $\begin{array}{l}\text { Downgrading } \\
\text { limitations in design: + } \\
\text { indirectness of evidence: - } \\
\text { unexplained heterogeneity: + } \\
\text { imprecision of results: - } \\
\text { high probability publication bias: } \\
\text { - } \\
\text { Upgrading: } \\
\text { absence of confounders: - } \\
\text { effect likely larger then found: - } \\
\text { dose-response gradient present: } \\
\text { - } \\
\text { Total: very low }\end{array}$ \\
\hline
\end{tabular}




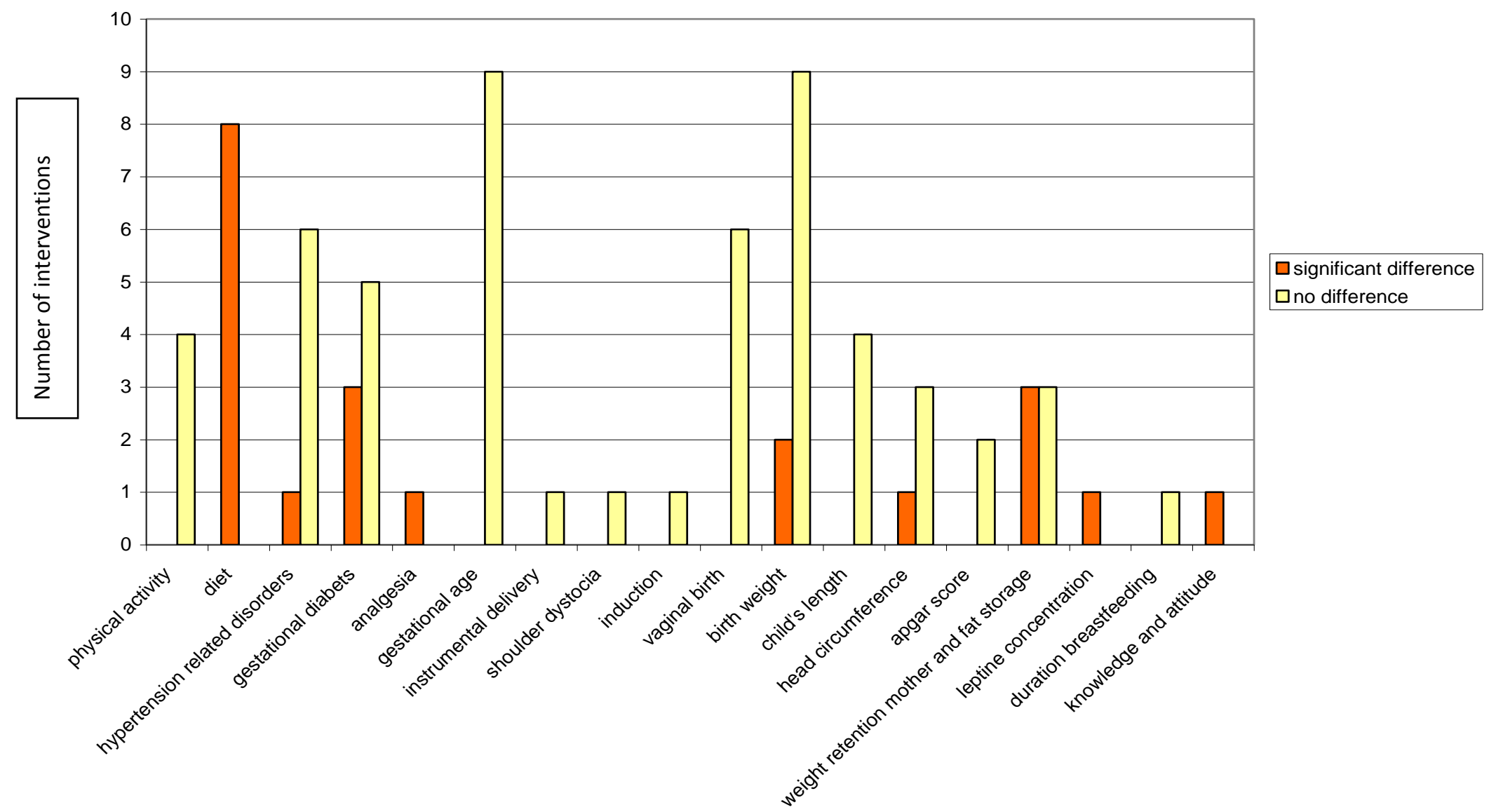

Figure 2.2 Secondary outcomes of interventions 
Table 7.2 Change objectives for pregnant women

Determinants $\rightarrow$ Awareness Knowledge

Attitudes

Social influences Self-efficacy/ skills

Barriers

\section{Performance}

objectives (PO)

\section{P01 Pregnant}

women determine

their BMI

k1a. Describe height and weight.
k1b Describe the calculation of

a1a. Believe it is important to

know their personal BMI

$\mathrm{BMI}$

\section{sila.Describe that se1a. Express}

midwives confidence in

consider knowing calculation of BMI

your BMI as (including

important measuring height

and weight)

si2a. Describe se2a. Express

that it is confidence in

reach a healthy GWG

a2b. Believe it is important to

protect their foetus' health

with a healthy GWG

a2c. Believe it is important to

protect their own health with

a healthy GWG

a2d. Believe it is important to

return to their pre-pregnancy

monitoring GWG

b2a. Express the

midwives'

usage of a scale

mbition to reach

a healthy GWG

si2b. Describe

that their midwife

stimulates them

k2d. Describe the healthy GWG

for their BMI at the end of

pregnancy

k2e. Describe the negative

consequences (short- and long-

term) of unhealthy GWG

weight after birth

a2e. Believe it is important to

monitor their GWG

$k 2 f$. Describe the positive

consequences (short and long-

term) of healthy GWG

PO3 Pregnant

women select ways

to accomplish

healthy GWG

$$
\text { aw3a. }
$$

healthy GWG k3a. Describe the contribution of

healthy diet to GWG

$k 3 b$. Describe the contribution of

healthy PA to GWG

$\mathrm{k} 3 \mathrm{c}$. Describe the contribution of

well-being to GWG

k3d. Describe the advantages of

monitoring GWG during

pregnancy

k3e. Describe the advantages of discussing unhealthy GWG with a3a. Feel positive about

reaching a healthy GWG with

a healthy diet

a3b. Feel positive about

reaching a healthy GWG with

healthy PA

3c. Feel positive about

reaching a healthy GWG with

well-being

a3d. Believe it is important to determine a personal target to monitor their

GWG

that a healthy

lifestyle (diet, PA)

is their midwives"

ambition

si3b. Describe

that their midwife

stimulates them

to reach a healthy

si3c. Describe se3a. Express

confidence in

realizing healthy

se3b. Express

confidence in

realizing healthy PA

se2c. Express

confidence in

protecting their own well-being. b3a. Believe that

they can handle changing dietary

b3b. Believe that they can maintain

healthy diet in case of physical complaints.

b3c. Believe that they can maintain 
the midwife.

K3f. describe frequently heard

well-meant untrue advices weight

a3e. Believe it is important to regularly monitor their GWG a3f. Believe it is important to discuss unhealthy GWG with the midwife that they feel supported by their midwives in making GWG negotiable si3d. Describe being able to resist social pressure ("eat for two", "stay inactive")

si3e. Describe being able to resist social

pressure of their offspring to snack unhealthy or to be inactive

\begin{tabular}{ll}
\hline PO4 Pregnant & aw4a. \\
women follow & Accord the value \\
midwives' advice & of following \\
about healthy GWG & midwives' advice \\
& about healthy
\end{tabular}

k4a. Recall midwives' advice
concerning GWG.

a4a. Believe it is important to follow midwives' advices a4b. Believe that midwives' advices belong to themselves

si4a. Describe

that others in

their

nvironment endorse The importance to

follow midwives' advices se3d. Demonstrate

discussing

unhealthy GWG

with their midwife

se3e. Express

confidence to resist

well-meant untrue

advices

se3f. Express

confidence in

resisting pressure

from their offspring healthy PA in case of physical

complaints.

b3d. Believe that they can have a

healthy lifestyle in combination with the care for their offspring.

se4a. Express
confidence in
following midwives'
advices

b4a. Plan how to cope with barriers that hinder

following

midwives' advices (tiredness, care

for offspring, time, etc.) b4b. Describe how they include midwives' advices into daily routine.

\begin{tabular}{llll}
\hline PO5 Pregnant & aw5a. Accord the & a5a. Believe it is important to & si5a. Recognize \\
women follow & value of visiting & visit, if relevant, a dietician, & that is normal to \\
midwives' advice & dietician, & psychologist, an exercise & visit a dietician, \\
about referral to & psychologist, an & program & psychologist, an \\
dietician, & exercise program & & exercise program
\end{tabular}

psychologist, an 
Table 7.3 Change objectives for midwives

Determinants $\rightarrow$ Awareness of Knowledge

Awareness of

Social influences

Self-efficacy/

Barriers

Performance role

objectives (PO)

\begin{tabular}{ll}
\hline PO1 Midwives & aw1a. \\
facilitate & Confirm the \\
information supply & value of \\
about healthy GWG & education \\
to all pregnant & about GWG \\
women in their first & on pregnant \\
trimester & $\begin{array}{l}\text { women's } \\
\text { behaviour }\end{array}$
\end{tabular}

a1a. Believe it is

important to stimulate

healthy GWG

a1b. Believe it is

important to provide

information about

healthy GWG

a1c. Believe it is

important to draw

pregnant women's

attention to strategies

for reaching healthy

GWG

a1d. Believe it is

important to inform

pregnant women about

healthy GWG

PO2 At a

gestational age of

about 16 weeks,

midwives adapt

their care

concerning healthy

weight gain to

individual needs of

pregnant women aw2a.

Confirm the

mpact of

societal

temptations

that inhibit

healthy GWG.

k2a. Describe advantages of healthy

GWG for pregnant women and their

ffspring (short- and long-term)

$\mathrm{K} 2 \mathrm{~b}$ Describe short- and long-term

consequences of unhealthy GWG

$\mathrm{K} 3 \mathrm{c}$.Describe ways to reach a healthy

2a. Believe it is

important to determine

-together with the

pregnant women- a

target weight for the

complete pregnancy

GWG (diet, PA, well-being in general).

3d. Describe the content of healthy

dietary behaviour

$k 2 e$. Describe the content of healthy

$k 2 f$. Describe the ingredients of well-

being in general (WHO definition:

physical, mental, societal well-being)

k2g. Describe for each BMI category
si2a.Recognize that pregnant

women expect them to

rovide education about

healthy GWG

\begin{tabular}{|c|c|}
\hline $\begin{array}{l}\text { se2a. Express } \\
\text { confidence in } \\
\text { matching BMI } \\
\text { categories with } \\
\text { specific target } \\
\text { weights and } \\
\text { specific GWG per } \\
\text { time episode } \\
\text { Se2b. } \\
\text { Demonstrate the } \\
\text { ability to combine } \\
\text { target weight } \\
\text { according the } \\
\text { guidelines with }\end{array}$ & $\begin{array}{l}\text { b2a. Plan how } \\
\text { to cope with } \\
\text { time barriers } \\
\text { that hinder } \\
\text { providing } \\
\text { GWG } \\
\text { education } \\
\text { B2b. Use a } \\
\text { protocol to } \\
\text { perform a } \\
\text { weight } \\
\text { anamnesis }\end{array}$ \\
\hline
\end{tabular}


the appropriate target weight and

healthy GWG per time episode (first

trimester, after first trimester weekly

GWG)

$k 2 h$. Describe that information supply

about GWG to pregnant women

promotes reaching healthy GWG

$k 2 i$. Describe a clear plan for the care

of GWG, diet and PA

k2j. Explain effective medical

communication (interactive,

effectual, etc.)

$k 2 k$. Explain the effect of goalsetting

with regard to GWG at the end of

\begin{tabular}{ll}
\hline PO3 Midwives & aw3a. \\
support pregnant & Confirm the \\
women with & value of \\
monitoring, & midwife's \\
appraisal of their & support on \\
GWG & GWG and \\
& well-being
\end{tabular}

\section{pregnancy}

k3a. Explain the contribution of monitoring and evaluating GWG during pregnancy

k3b. Describe how to monitor and evaluate GWG

\section{a3a. Believe it is important to stimulate pregnant women to \\ monitor and evaluate \\ their GWG \\ a3b. Believe it is \\ important that pregnant \\ women take \\ responsibility for their \\ GWG \\ a3c. Believe it is \\ important to show \\ interest in pregnant women's GWG}

target weight of a pregnant woman.

Se2c. Feel

confidence in

bringing healthy

GWG under

attention of

pregnant $\mathrm{OW} / \mathrm{OB}$

women
PO4 Midwives

identify clients who

are at greater risk

for unhealthy GWG k4a. Describe risk groups for gaining unhealthy GWG.

k4b. Describe adequate weight

anamneses

K4c. Describe an healthy weight curve and the dangers of deviation from the curve

a4a. Believe it is

important to identify

those pregnant women

who need support

a4b. Believe it is

important to carry out

an adequate weight

anamnesis

4c. Believe it is si3a. Believe that pregnant women think it is important

to monitoring their (pregnant women's) GWG

se3a. Express

confidence in

supporting

pregnant women

in monitoring and

evaluating GWG

se3b.

Demonstrate the

ability to pro-

actively ask

pregnant women

to their GWG

se4a

Demonstrate the

ability to take an

adequate weight

anamnesis

se4b. Express

confidence in

being able to

detect a deviation b4a. Plan

sufficient

time to

perform an

adequate

weight

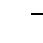




\begin{tabular}{|c|c|c|c|c|c|c|}
\hline $\begin{array}{l}\text { PO5 Midwives } \\
\text { empower those } \\
\text { clients who are at } \\
\text { greater risk for } \\
\text { unhealthy GWG in } \\
\text { reaching a healthy } \\
\text { GWG }\end{array}$ & $\begin{array}{l}\text { aw5a. } \\
\text { Acknowledge } \\
\text { the relation } \\
\text { between } \\
\text { GWG/ } \\
\text { lifestyles and } \\
\text { pregnant } \\
\text { women's } \\
\text { well- being } \\
\text { aw5b. } \\
\text { Confirm the } \\
\text { value of } \\
\text { midwife's } \\
\text { support } \\
\text { aw5c. } \\
\text { Confirm the } \\
\text { value of other } \\
\text { professionals' } \\
\text { contribution } \\
\text { in regard to } \\
\text { GWG }\end{array}$ & $\begin{array}{l}\text { k5a. Describe pregnancy complaints } \\
\text { that increase the chance on } \\
\text { unhealthy GWG }\end{array}$ & $\begin{array}{l}\text { a5a. Believe it is } \\
\text { important to support } \\
\text { pregnant women in } \\
\text { reaching a healthy GWG } \\
\text { a5b. Express openness } \\
\text { to questions and } \\
\text { concerns from pregnant } \\
\text { women about their } \\
\text { GWG/ lifestyle } \\
\text { a5c. Believe it is } \\
\text { important to search, } \\
\text { together with the } \\
\text { pregnant woman, for } \\
\text { causes and solutions for } \\
\text { GWG/ lifestyle related } \\
\text { problems in order to } \\
\text { retrieve a healthy GWG } \\
\text { a5d.Believe it is } \\
\text { important to refer } \\
\text { pregnant women, if } \\
\text { necessary, to } \\
\text { professionals } \\
\text { specialized in diet, } \\
\text { exercise, well-being. } \\
\text { a5e. Express positive } \\
\text { feelings towards } \\
\text { engagement in } \\
\text { collaborative } \\
\text { partnership }\end{array}$ & $\begin{array}{l}\text { si5a. Recognize that other } \\
\text { midwives think that } \\
\text { midwives' support in } \\
\text { reaching healthy GWG is } \\
\text { important } \\
\text { si5b. Recognize that } \\
\text { pregnant women think that } \\
\text { midwives' support in } \\
\text { reaching healthy GWG is } \\
\text { important } \\
\text { si5c. Recognize that pregnant } \\
\text { women think it is important } \\
\text { to find ways to reach healthy } \\
\text { GWG } \\
\text { si5d. Recognize that } \\
\text { colleagues think it is } \\
\text { important to find ways to } \\
\text { reach healthy GWG }\end{array}$ & $\begin{array}{l}\text { se5a. Express } \\
\text { confidence in } \\
\text { supporting } \\
\text { pregnant women } \\
\text { to a healthy } \\
\text { GWG/ lifestyle } \\
\text { Se5b. Express } \\
\text { confidence in } \\
\text { searching with } \\
\text { pregnant women } \\
\text { for causes for } \\
\text { deviation from } \\
\text { the curve } \\
\text { Se5c. Express } \\
\text { confidence in } \\
\text { searching with } \\
\text { pregnant women } \\
\text { to solutions for } \\
\text { deviation from } \\
\text { the curve } \\
\text { Se5d. Express } \\
\text { confidence in } \\
\text { supplying advices } \\
\text { about diet, } \\
\text { exercise and well- } \\
\text { being in general } \\
\text { Se5e. Express } \\
\text { confidence in } \\
\text { discussing the } \\
\text { GWG curve }\end{array}$ & $\begin{array}{l}\text { b5a. Plan } \\
\text { sufficient } \\
\text { time to } \\
\text { discuss with } \\
\text { pregnant } \\
\text { women } \\
\text { healthy } \\
\text { GWG/ } \\
\text { lifestyle and } \\
\text { ways how to } \\
\text { achieve it } \\
\text { B5b. Make } \\
\text { use of } \\
\text { practical } \\
\text { tools/ } \\
\text { guidelines for } \\
\text { the coaching } \\
\text { of a healthy } \\
\text { GWG/ } \\
\text { lifestyle } \\
\text { b5c. Plan } \\
\text { sufficient } \\
\text { time to coach } \\
\text { pregnant } \\
\text { women to } \\
\text { healthy } \\
\text { GWG/ } \\
\text { lifestyle } \\
\text { b5d. Plan } \\
\text { sufficient } \\
\text { time to } \\
\text { discuss } \\
\text { causes with } \\
\text { pregnant } \\
\text { women who }\end{array}$ \\
\hline
\end{tabular}




\section{P06 Midwives}

identify those

pregnant women

who need special

care, which exceeds

midwives"

capabilities

P07 Midwives refer

pregnant women, if

necessary to other

health care

professionals

k6a. Describe criteria for referral to

specialized care givers

$\mathrm{K} 6 \mathrm{~b}$. Explain that gaining unhealthy

GWG necessitates specialized care

important to identify

those pregnant wome

who need support of

other specialized

professionals.

b6a. Use

guidelines to

perform

adequate

lifestyle

anamnesis

$\begin{array}{ll}\begin{array}{l}\text { se7a. Express } \\ \text { confidence to } \\ \text { refer to } \\ \text { professionals } \\ \text { specialized in diet, } \\ \text { PA, exercise }\end{array} & \begin{array}{l}\text { b7a. Prepare/ } \\ \text { adapt a list } \\ \text { of eligible } \\ \text { local } \\ \text { programs }\end{array} \\ \begin{array}{l}\text { healthcare } \\ \text { professionals }\end{array} \\ \begin{array}{l}\text { confidence to } \\ \text { support }\end{array} \\ \begin{array}{l}\text { professionals } \\ \text { specialized in diet, } \\ \text { PA, exercise }\end{array} \\ \text { programs. }\end{array}$

\section{PO8 In case of}

referral of pregnant

women to other

healthcare

professionals,

midwives support

the care of those

programs.

professionals 


\section{References}

1. Fontein Kuipers YJ. WazzUp Mama?! The development of an intervention to prevent and reduce maternal distress during pregnancy. Maastricht: Universiteit van Maastricht; 2016.

2. Rode L, Kjaergaard H, Ottesen B, Damm P, Hegaard HK. Association between gestational weight gain according to body mass index and postpartum weight in a large cohort of Danish women. Matern Child Health J. 2012 Feb;16(2):406-13.

3. McClure CK, Catov JM, Ness R, Bodnar LM. Associations between gestational weight gain and BMI, abdominal adiposity, and traditional measures of cardiometabolic risk in mothers 8 y postpartum. Am J Clin Nutr. 2013 Nov;98(5):1218-25.

4. Symonds ME, Mendez MA, Meltzer HM, Koletzko B, Godfrey K, Forsyth S, et al. Early life nutritional programming of obesity: mother-child cohort studies. Ann Nutr Metab. 2013;62(2):137-45.

5. Koletzko B, Brands B, Poston L, Godfrey K, Demmelmair H. Early nutrition programming of long-term health. Proc Nutr Soc. 2012 Aug;71(3):371-8.

6. Rasmussen KM, Yaktine AL. Weight gain during pregnancy, reexamining the guidelines. Washington: Institute Of Medicine, 2009.

7. World Health Organisation. Obesity and overweight, factsheet no 311. [Internet]. 2013 [cited 13-092013]. Available from: http://www.who.int/mediacentre/factsheets/fs311/en/index.html.

8. Wallenburg HCS, Haan Jd, Huisjes HJ. De normale zwangerschap [Normal pregnancy]. In: Kloosterman GJ, editor. De voortplanting van de mens, leerboek voor obstetrie en gynaecologie [Human procreation, studybook for obstetry and gynaecology]. 7 ed. Weesp: Centen; 1985.

9. Jebeile H, Mijatovic J, Louie JC, Prvan T, Brand Miller JC. A systematic review and meta-analysis of energy intake and weight gain in pregnancy. Am J Obstet Gyneco;214(4): 465-83.

10. Orsama AL, Mattila E, Ermes M, van Gils M, Wansink B, Korhonen I. Weight rhythms: weight increases during weekends and decreases during weekdays. Obes Facts. 2014;7(1):36-47.

11. Keller C, Siegrist M. Ambivalence toward palatable food and emotional eating predict weight fluctuations. Results of a longitudinal study with four waves. Appetite. 2015 Feb;85:138-45.

12. van Strien T, Herman CP, Verheijden MW. Dietary restraint and body mass change. A 3-year follow up study in a representative Dutch sample. Appetite. 2014 May;76:44-9.

13. Rasmussen KM, Catalano PM, Yaktine AL. New guidelines for weight gain during pregnancy: what obstetrician/gynecologists should know. Current opinion in obstetrics \& gynecology. 2009 Dec;21(6):521-6.

14. Institute of Medicine. Nutrition during pregnancy. Washington: Institute of Medicine, 1990.

15. Mohangoo AD, Buitendijk SE, Hukkelhoven CW, Ravelli AC, Rijninks van Driel GC, Tamminga P, et al. Hoge perinatale sterfte in Nederland vergeleken met andere Europese landen: de Peristat-II-studie. Ned Tijdschr Geneeskd. 2008 Dec 13;152(50):2718-27.

16. Mohangoo AD, Buitendijk SE, Szamotulska K, Chalmers J, Irgens LM, Bolumar F, et al. Gestational age patterns of fetal and neonatal mortality in Europe: results from the Euro-Peristat project. PLoS One.

2011;6(11):e24727.

17. Bonsel GJ, Birnie E, Denktas S, Poeran J, Steegers EAP. Lijnen in de Perinatale sterfte, Signalementstudie 'Zwangerschap en Geboorte' 2010 [Evalution study of perintal death]. Rotterdam: Erasmus MC, 2010.

18. Siega Riz AM, Viswanathan M, Moos MK, Deierlein A, Mumford S, Knaack J, et al. A systematic review of outcomes of maternal weight gain according to the Institute of Medicine recommendations: birthweight, fetal growth, and postpartum weight retention. Am J Obstet Gynecol. 2009 Oct;201(4):339.e1-14.

19. Viswanathan M, Siega Riz AM, Moos MK, Deierlein A, Mumford S, Knaack J, et al. Outcomes of maternal weight gain. Evid Rep Technol Assess (Full Rep). 2008 May(168):1-223.

20. Nehring I, Schmoll S, Beyerlein A, Hauner H, von Kries R. Gestational weight gain and long-term postpartum weight retention: a meta-analysis. Am J Clin Nutr. 2011 Nov;94(5):1225-31.

21. Lau EY, Liu J, Archer E, McDonald SM, Liu J. Maternal weight gain in pregnancy and risk of obesity among offspring: a systematic review. J Obes;2014 (2014):524939:.

22. Adamo KB, Ferraro ZM, Brett KE. Can We Modify the Intrauterine Environment to Halt the Intergenerational Cycle of Obesity? International Journal of Environmental Research and Public Health. 2012;9(12):1263-307.

23. Poston L. Maternal obesity, gestational weight gain and diet as determinants of offspring long term health. Best Pract Res Clin Endocrinol Metab. 2012 Oct;26(5):627-39. 
24. Poston L. Gestational weight gain: influences on the long-term health of the child. Curr Opin Clin Nutr Metab Care. 2012 May;15(3):252-7.

25. Mamun AA, Mannan M, Doi SA. Gestational weight gain in relation to offspring obesity over the life course: a systematic review and bias-adjusted meta-analysis. Obes Rev. 2014 Apr;15(4):338-47.

26. Nehring I, Lehmann S, von Kries R. Gestational weight gain in accordance to the IOM/NRC criteria and the risk for childhood overweight: a meta-analysis. Pediatr Obes. 2013 Jun;8(3):218-24.

27. McDonald SD, Han Z, Mulla S, Lutsiv O, Lee T, Beyene J. High gestational weight gain and the risk of preterm birth and low birth weight: a systematic review and meta-analysis. J Obstet Gynaecol Can. 2011 Dec;33(12):1223-33.

28. Kapadia MZ, Gaston A, Van Blyderveen S, Schmidt L, Beyene J, McDonald H, et al. Psychological factors and trimester-specific gestational weight gain: a systematic review. J Psychosom Obstet Gynaecol. 2015;36(1):1522.

29. Kapadia MZ, Gaston A, Van Blyderveen S, Schmidt L, Beyene J, McDonald H, et al. Psychological antecedents of excess gestational weight gain: a systematic review. BMC Pregnancy Childbirth. 2015;15:107.

30. Han Z, Lutsiv O, Mulla S, Rosen A, Beyene J, McDonald SD. Low gestational weight gain and the risk of preterm birth and low birthweight: a systematic review and meta-analyses. Acta Obstet Gynecol Scand. 2011 Sep;90(9):935-54.

31. Ruifrok AE, van Poppel MN, van Wely M, Rogozinska E, Khan KS, de Groot CJ, et al. Association between weight gain during pregnancy and pregnancy outcomes after dietary and lifestyle interventions: a meta-analysis. Am J Perinatol. 2014 May;31(5):353-64.

32. O'Brien CM, Grivell RM, Dodd JM. Systematic review of antenatal dietary and lifestyle interventions in women with a normal body mass index. Acta Obstet Gynecol Scand. 2016 Mar;95(3):259-69.

33. Zhang CH, Liu XY, Zhan YW, Zhang L, Huang YJ, Zhou H. Effects of Prepregnancy Body Mass Index and Gestational Weight Gain on Pregnancy Outcomes. Asia Pac J Public Health. 2015 Sep;27(6):620-30.

34. Morken NH, Klungsoyr K, Magnus P, Skjaerven R. Pre-pregnant body mass index, gestational weight gain and the risk of operative delivery. Acta Obstet Gynecol Scand. 2013 Jul;92(7):809-15.

35. Crane JM, White J, Murphy P, Burrage L, Hutchens D. The effect of gestational weight gain by body mass index on maternal and neonatal outcomes. J Obstet Gynaecol Can. 2009 Jan;31(1):28-35.

36. Pop VJ, Biondi B, Wijnen HA, Kuppens SM, Lvader H. Maternal thyroid parameters, body mass index and subsequent weight gain during pregnancy in healthy euthyroid women. Clin Endocrinol (Oxf). 2013 Oct;79(4):577-

83.

37. Liu Y, Dai W, Dai X, Li Z. Prepregnancy body mass index and gestational weight gain with the outcome of pregnancy: a 13-year study of 292,568 cases in China. Arch Gynecol Obstet. 2012 Oct;286(4):905-11.

38. Dodd JM, McPhee AJ, Turnbull D, Yelland LN, Deussen AR, Grivell RM, et al. The effects of antenatal dietary and lifestyle advice for women who are overweight or obese on neonatal health outcomes: the LIMIT randomised trial. BMC Med. 2014;12:163.

39. Hrolfsdottir L, Rytter D, Olsen SF, Bech BH, Maslova E, Henriksen TB, et al. Gestational weight gain in normal weight women and offspring cardio-metabolic risk factors at 20 years of age. Int J Obes (Lond). 2015 Apr;39(4):671-6.

40. Villamor E, Cnattingius S. Interpregnancy weight change and risk of adverse pregnancy outcomes: a population-based study. Lancet. 2006 Sep 30;368(9542):1164-70.

41. Hunt KJ, Alanis MC, Johnson ER, Mayorga ME, Korte JE. Maternal pre-pregnancy weight and gestational weight gain and their association with birthweight with a focus on racial differences. Matern Child Health J. 2013 Jan;17(1):85-94.

42. Simas TA, Liao X, Garrison A, Sullivan GM, Howard AE, Hardy JR. Impact of updated Institute of Medicine guidelines on prepregnancy body mass index categorization, gestational weight gain recommendations, and needed counseling. J Womens Health (Larchmt). 2011 Jun;20(6):837-44.

43. Hill BESS, Skouteris HAPP, McCabe MPP, Milgrom JPP, Kent BPP, Herring SJAPMD, et al. A conceptual model of psychosocial risk and protective factors for excessive gestational weight gain. Midwifery. 2013;29(2):1104.

44. Althuizen E, van Poppel MN, Seidell JC, van Mechelen W. Correlates of absolute and excessive weight gain during pregnancy. J Womens Health (Larchmt). 2009 Oct;18(10):1559-66.

45. Daemers DO, Wijnen HA, van Limbeek EB, Bude LM, de Vries RG. Patterns of gestational weight gain in healthy, low-risk pregnant women without co-morbidities. Midwifery. 2013 May;29(5):535-41.

46. Spelten ER, Klomp T, Mannien J. Deliver-studie 2008-2011, National studie naar de eerstelijns verloskunde [National study of primary midwifery care] Academie Verloskunde Amsterdam Groningen; 2011. 47. Walker LO. Managing excessive weight gain during pregnancy and the postpartum period. J Obstet Gynecol Neonatal Nurs. 2007 Sep-Oct;36(5):490-500. 
48. Pawlak MT, Alvarez BT, Jones DM, Lezotte DC. The effect of race/ethnicity on gestational weight gain. J Immigr Minor Health. 2015 Apr;17(2):325-32.

49. Holowko N, Chaparro MP, Nilsson K, Ivarsson A, Mishra G, Koupil I, et al. Social inequality in prepregnancy BMI and gestational weight gain in the first and second pregnancy among women in Sweden. J Epidemiol Community Health. 2015 Dec;69(12):1154-61.

50. Holowko N, Mishra G, Koupil I. Social inequality in excessive gestational weight gain. Int J Obes (Lond). 2014 Jan;38(1):91-6.

51. Olson CM, Strawderman MS. Modifiable behavioral factors in a biopsychosocial model predict inadequate and excessive gestational weight gain. J Am Diet Assoc. 2003 Jan;103(1):48-54.

52. Ashley Martin J, Woolcott C. Gestational weight gain and postpartum weight retention in a cohort of Nova Scotian women. Matern Child Health J. 2014 Oct;18(8):1927-35.

53. Restall A, Taylor RS, Thompson JM, Flower D, Dekker GA, Kenny LC, et al. Risk factors for excessive gestational weight gain in a healthy, nulliparous cohort. J Obes:2014(2014):148391.

54. Provenzano AM, Rifas Shiman SL, Herring SJ, Rich Edwards JW, Oken E. Associations of maternal material hardships during childhood and adulthood with prepregnancy weight, gestational weight gain, and postpartum weight retention. J Womens Health (Larchmt). 2015 Jul;24(7):563-71.

55. Alhusen JL, Ray E, Sharps P, Bullock L. Intimate partner violence during pregnancy: maternal and neonatal outcomes. J Womens Health (Larchmt). 2015 Jan;24(1):100-6.

56. Headen IE, Davis EM, Mujahid MS, Abrams B. Racial-ethnic differences in pregnancy-related weight. Adv Nutr. 2012 Jan;3(1):83-94.

57. Herring SJ, Nelson DB, Davey A, Klotz AA, Dibble LV, Oken E, et al. Determinants of excessive gestational weight gain in urban, low-income women. Womens Health Issues. 2012 Sep;22(5):e439-46.

58. Muktabhant B, Lawrie TA, Lumbiganon P, Laopaiboon M. Diet or exercise, or both, for preventing excessive weight gain in pregnancy. Cochrane Database Syst Rev. 2015 Jun 11;6:CD007145.

59. Stotland NE, Haas JS, Brawarsky P, Jackson RA, Fuentes Afflick E, Escobar GJ. Body mass index, provider advice, and target gestational weight gain. Obstet Gynecol. 2005 Mar;105(3):633-8.

60. Cohen TR, Koski KG. Limiting excess weight gain in healthy pregnant women: importance of energy intakes, physical activity, and adherence to gestational weight gain guidelines. J Pregnancy. 2013;2013:787032. 61. de Jersey SJ, Nicholson JM, Callaway LK, Daniels LA. A prospective study of pregnancy weight gain in Australian women. Aust N Z J Obstet Gynaecol. 2012 Dec;52(6):545-51.

62. Bartholomew L, Parcel G, Kok G, Gottlieb N, Fernandez M. Planning Health Promotion Programs: An Intervention Mapping Approach. San Francisco: Jossey-Bass; 2011.

63. Gardner B, Wardle J, Poston L, Croker H. Changing diet and physical activity to reduce gestational weight gain: a meta-analysis. Obes Rev. $2011 \mathrm{Jul} ; 12(7)$ :e602-20.

64. Kuhlmann AK, Dietz PM, Galavotti C, England L. Weight-management interventions for pregnant or postpartum women. Am J Prev Med. 2008 Jun;34(6):523-8.

65. Birdsall KM, Vyas S, Khazaezadeh N, Oteng Ntim E. Maternal obesity: a review of interventions. Int J Clin Pract. 2009 Mar;63(3):494-507.

66. Nascimento SL, Surita FG, Cecatti JG. Physical exercise during pregnancy: a systematic review. Current opinion in obstetrics \& gynecology. 2012 Dec;24(6):387-94.

67. Dodd JM, Grivell RM, Crowther CA, Robinson JS. Antenatal interventions for overweight or obese pregnant women: a systematic review of randomised trials. BJOG. 2010 Oct;117(11):1316-26.

68. Morisset AS, St-Yves A, Veillette J, Weisnagel SJ, Tchernof A, Robitaille J. Prevention of gestational diabetes mellitus: a review of studies on weight management. Diabetes/metabolism research and reviews. 2010 Jan;26(1):17-25.

69. Nielsen JN, Gittelsohn J, Anliker J, O'Brien K. Interventions to improve diet and weight gain among pregnant adolescents and recommendations for future research. J Am Diet Assoc. 2006 Nov;106(11):1825-40. 70. Shirazian T, Raghavan S. Obesity and pregnancy: implications and management strategies for providers. Mt Sinai J Med. 2009 Dec;76(6):539-45.

71. Skouteris H, Hartley-Clark L, McCabe M, Milgrom J, Kent B, Herring SJ, et al. Preventing excessive gestational weight gain: a systematic review of interventions. Obes Rev. 2010 Nov;11(11):757-68.

72. Campbell F, Johnson M, Messina J, Guillaume L, Goyder E. Behavioural interventions for weight management in pregnancy: a systematic review of quantitative and qualitative data. BMC public health. 2011;11:491.

73. Imdad A, Bhutta ZA. Effect of balanced protein energy supplementation during pregnancy on birth outcomes. BMC public health. 2011;11(3):1.

74. Kramer MS, Kakuma R. Energy and protein intake in pregnancy. Cochrane Database Syst Rev. 2003

(4):Cd000032. 
75. Streuling I, Beyerlein A, Rosenfeld E, Hofmann H, Schulz T, von Kries R. Physical activity and gestational weight gain: a meta-analysis of intervention trials. BJOG. 2011 Feb;118(3):278-84.

76. Widen E, Siega Riz AM. Prenatal nutrition: a practical guide for assessment and counseling. J Midwifery Womens Health. 2010 Nov-Dec;55(6):540-9.

77. Olson CM. Achieving a healthy weight gain during pregnancy. Annu Rev Nutr. 2008;28:411-23.

78. Ronnberg AK, Nilsson K. Interventions during pregnancy to reduce excessive gestational weight gain: a systematic review assessing current clinical evidence using the Grading of Recommendations, Assessment, Development and Evaluation (GRADE) system. BJOG. 2010 Oct;117(11):1327-34.

79. Higgins JP, Green S. Cochrane handbook for systematic reviews of interventions: Wiley Online Library; 2008.

80. Schulz KF, Altman DG, Moher D. CONSORT 2010 statement: updated guidelines for reporting parallel group randomised trials. BMC medicine. 2010;8(1):1.

81. Moher D, Hopewell S, Schulz KF, Montori V, Gotzsche PC, Devereaux PJ, et al. CONSORT 2010 explanation and elaboration: updated guidelines for reporting parallel group randomised trials. BMJ (Clinical research ed). 2010; Mar 23;340:c869.

82. Verhagen AP, de Vet HC, de Bie RA, Kessels AG, Boers M, Bouter LM, et al. The Delphi list: a criteria list for quality assessment of randomized clinical trials for conducting systematic reviews developed by Delphi consensus. J Clin Epidemiol. 1998 Dec;51(12):1235-41.

83. Macedo LG, Elkins MR, Maher CG, Moseley AM, Herbert RD, Sherrington C. There was evidence of convergent and construct validity of Physiotherapy Evidence Database quality scale for physiotherapy trials. J Clin Epidemiol. 2010 Aug;63(8):920-5.

84. Jackson RA, Stotland NE, Caughey AB, Gerbert B. Improving diet and exercise in pregnancy with Video Doctor counseling: a randomized trial. Patient Educ Couns. 2011 May;83(2):203-9.

85. Riffenburgh RH. Statistics in medicine. San Diego, CA :: Academic Press; 1999.

86. Phelan S, Phipps MG, Abrams B, Darroch F, Schaffner A, Wing RR. Randomized trial of a behavioral intervention to prevent excessive gestational weight gain: the Fit for Delivery Study. Am J Clin Nutr. 2011 Apr;93(4):772-9.

87. Quinlivan JA, Lam LT, Fisher J. A randomised trial of a four-step multidisciplinary approach to the antenatal care of obese pregnant women. Aust N Z J Obstet Gynaecol. 2011 Apr;51(2):141-6.

88. Asbee SM, Jenkins TR, Butler JR, White J, Elliot M, Rutledge A. Preventing excessive weight gain during pregnancy through dietary and lifestyle counseling: a randomized controlled trial. Obstet Gynecol. 2009 Feb;113(2 Pt 1):305-12.

89. de Barros MC, Lopes MA, Francisco RP, Sapienza AD, Zugaib M. Resistance exercise and glycemic control in women with gestational diabetes mellitus. Am J Obstet Gynecol. 2010 Dec;203(6):556.e1-6.

90. Barakat R, Lucia A, Ruiz JR. Resistance exercise training during pregnancy and newborn's birth size: a randomised controlled trial. Int J Obes (Lond). 2009 Sep;33(9):1048-57.

91. Cavalcante SR, Cecatti JG, Pereira RI, Baciuk EP, Bernardo AL, Silveira C. Water aerobics II: maternal body composition and perinatal outcomes after a program for low risk pregnant women. Reprod Health. 2009;6:1.

92. Guelinckx I, Devlieger R, Mullie P, Vansant G. Effect of lifestyle intervention on dietary habits, physical activity, and gestational weight gain in obese pregnant women: a randomized controlled trial. Am J Clin Nutr. 2010 Feb;91(2):373-80.

93. Hui AL, Ludwig S, Gardiner P, Sevenhuysen G, Murray R, Morris M, et al. Community-based exercise and dietary intervention during pregnancy: a pilot study. Canadian Journal of diabetes. 2006;30(2):1-7.

94. IImonen J, Isolauri E, Poussa T, Laitinen K. Impact of dietary counselling and probiotic intervention on maternal anthropometric measurements during and after pregnancy: a randomized placebo-controlled trial. Clin Nutr. 2011 Apr;30(2):156-64.

95. Luoto R, Kinnunen TI, Aittasalo M, Kolu P, Raitanen J, Ojala K, et al. Primary prevention of gestational diabetes mellitus and large-for-gestational-age newborns by lifestyle counseling: a cluster-randomized controlled trial. PLoS Med. 2011 May;8(5):e1001036.

96. Polley BA, Wing RR, Sims CJ. Randomized controlled trial to prevent excessive weight gain in pregnant women. Int J Obes Relat Metab Disord. 2002 Nov;26(11):1494-502.

97. Rhodes ET, Pawlak DB, Takoudes TC, Ebbeling CB, Feldman HA, Lovesky MM, et al. Effects of a lowglycemic load diet in overweight and obese pregnant women: a pilot randomized controlled trial. Am J Clin Nutr. 2010 Dec;92(6):1306-15.

98. Rosen T, de Veciana M, Miller HS, Stewart L, Rebarber A, Slotnick RN. A randomized controlled trial of nerve stimulation for relief of nausea and vomiting in pregnancy. Obstet Gynecol. 2003 Jul;102(1):129-35. 
99. Wolff S, Legarth J, Vangsgaard K, Toubro S, Astrup A. A randomized trial of the effects of dietary counseling on gestational weight gain and glucose metabolism in obese pregnant women. Int J Obes (Lond). 2008 Mar;32(3):495-501.

100. Bechtel Blackwell DA. Computer-assisted self-interview and nutrition education in pregnant teens. Clin Nurs Res. 2002 Nov;11(4):450-62.

101. Jeffries K, Shub A, Walker SP, Hiscock R, Permezel M. Reducing excessive weight gain in pregnancy: a randomised controlled trial. Med J Aust. 2009 Oct 19;191(8):429-33.

102. Haakstad LA, Bo K. Effect of regular exercise on prevention of excessive weight gain in pregnancy: a randomised controlled trial. Eur J Contracept Reprod Health Care. 2011 Apr;16(2):116-25.

103. Thornton YS. Smarkola C, Kopacz SM, Ishoof SB. Perinatal outcomes in nutritionally monitored obese pregnant women: a randomized clinical trial. J of the Nat Med Ass;101(6):569-77.

104. Kinnunen TI, Pasanen M, Aittasalo M, Fogelholm M, Hilakivi Clarke L, Weiderpass E, et al. Preventing excessive weight gain during pregnancy - a controlled trial in primary health care. Eur J Clin Nutr. 2007 Jul;61(7):884-91.

105. Shirazian T, Monteith S, Friedman F, Rebarber A. Lifestyle modification program decreases pregnancy weight gain in obese women. American journal of perinatology. 2010;27(05):411-4.

106. Artal R, Catanzaro RB, Gavard JA, Mostello DJ, Friganza JC. A lifestyle intervention of weight-gain restriction: diet and exercise in obese women with gestational diabetes mellitus. Appl Physiol Nutr Metab. 2007 Jun;32(3):596-601.

107. Claesson IM, Sydsjo G, Brynhildsen J, Cedergren M, Jeppsson A, Nystrom F, et al. Weight gain restriction for obese pregnant women: a case-control intervention study. BJOG. 2008 Jan;115(1):44-50.

108. Garg A, Kashyap S. Effect of counseling on nutritional status during pregnancy. Indian J Pediatr. 2006 Aug;73(8):687-92.

109. Klima C, Norr K, Vonderheid S, Handler A. Introduction of CenteringPregnancy in a public health clinic. J Midwifery Womens Health. 2009 Jan-Feb;54(1):27-34.

110. McDonough Catto J-E. Birth outcomes for high-risk pregnant medicaid women case managed by registered nurses 2003.

111. Mottola MF, Giroux I, Gratton R, Hammond JA, Hanley A, Harris S, et al. Nutrition and exercise prevent excess weight gain in overweight pregnant women. Med Sci Sports Exerc. 2010 Feb;42(2):265-72.

112. Olson CM, Strawderman MS, Reed RG. Efficacy of an intervention to prevent excessive gestational weight gain. Am J Obstet Gynecol. 2004 Aug;191(2):530-6.

113. Ruchat S-M, Sopper M, Giroux I, Davenport M, Mottola M. 171-Early intervention in overweight and obese gestational diabetic women may prevent excessive gestational weight gain and weight retention. Canadian Journal of Diabetes. 2011;35(2):184.

114. Marcus SM. Depression during pregnancy: rates, risks and consequences--Motherisk Update 2008. Can J Clin Pharmacol. 2009 Winter;16(1):e15-22.

115. Olson G, Blackwell SC. Optimization of gestational weight gain in the obese gravida: a review. Obstet Gynecol Clin North Am. 2011 Jun;38(2):397-407, xii.

116. Guelinckx I, Devlieger R, Beckers K, Vansant G. Maternal obesity: pregnancy complications, gestational weight gain and nutrition. Obes Rev. 2008 Mar;9(2):140-50.

117. Hui A, Back L, Ludwig S, Gardiner P, Sevenhuysen G, Dean H, et al. Lifestyle intervention on diet and exercise reduced excessive gestational weight gain in pregnant women under a randomised controlled trial. BJOG. 2012 Jan;119(1):70-7.

118. Nascimento SL, Surita FG, Parpinelli MA, Siani S, Pinto e Silva JL. The effect of an antenatal physical exercise programme on maternal/perinatal outcomes and quality of life in overweight and obese pregnant women: a randomised clinical trial. BJOG. 2011 Nov;118(12):1455-63.

119. Rosenbloom L, Buchert E, Vasiloff R, Feinglass J, Dong X, Simon M. Preventing excessive weight gain among publicly insured pregnant women. J Community Health. 2012 Oct;37(5):1066-70.

120. Della Libera B, Baiao MR, Santos MMDS, Padilha P, Alves PD, Saunders C. Adherence of pregnant women to dietary counseling and adequacy of total gestational weight gain. Nutr Hosp. 2011;26(1):79-85.

121. Melzer K, Schutz Y. Pre-pregnancy and pregnancy predictors of obesity. International journal of obesity (2005). 2010;34(Suppl 2):S44-52.

122. Yu Z, Han S, Zhu J, Sun X, Ji C, Guo X. Pre-pregnancy body mass index in relation to infant birth weight and offspring overweight/obesity: a systematic review and meta-analysis. PLoS One. 2013;8(4):e61627.

123. Pereboom MT, Manniën J, van Almkerk KD, Spelten ER, Gitsels JT, Martin L, et al. What information do Dutch midwives give clients about toxoplasmosis, listeriosis and cytomegalovirus prevention? An exploratory study of videotaped consultations. Patient education and counseling. 2014;96(1):29-35. 
124. Alavi N, Haley S, Chow K, McDonald SD. Comparison of national gestational weight gain guidelines and energy intake recommendations. Obes Rev. 2013 Jan;14(1):68-85.

125. Davenport MH, Ruchat S-M, Giroux I, Sopper MM, Mottola MF. Timing of excessive pregnancy-related weight gain and offspring adiposity at birth. Obstetrics and Gynecology. 2013 (122):7..

126. Rauh K, Gabriel E, Kerschbaum E, Schuster T, von Kries R, Amann-Gassner U, et al. Safety and efficacy of a lifestyle intervention for pregnant women to prevent excessive maternal weight gain: a cluster-randomized controlled trial. BMC Pregnancy Childbirth. 2013;13:151.

127. Agudo A. Measuring intake of fruit and vegetables: [Internet]. 2016 [cited 6-12-2016]. Available from: http://www.who.int/dietphysicalactivity/publications/en/.

128. World Health Organization. Global recommendations on physical activity for health. WHO, 2010.

129. Van Rossum CTM, Fransen HP, Verkaik-Kloosterman J, Buurma-Rethans EJM, Ocke M. Dutch National Food Consumption Survey 2007-2010: Diet of Children and Adults Aged 7 to 69 Years RIVM, [Internet]. 2011 [cited 18-3-2016]. Available from: http://www.rivm.nl/bibliotheek/rapporten/350050006.pdf.

130. Hildebrandt V, Bernaards C, Stubbe J. Trendrapport bewegen en gezondheid [Trends in physical activity and health]. Leiden: TNO, 2013.

131. Adegboye AR, Rossner S, Neovius M, Lourenco PM, Linne Y. Relationships between prenatal smoking cessation, gestational weight gain and maternal lifestyle characteristics. Women and birth : journal of the Australian College of Midwives. 2010 Mar;23(1):29-35.

132. Olafsdottir AS, Skuladottir GV, Thorsdottir I, Hauksson A, Steingrimsdottir L. Combined effects of maternal smoking status and dietary intake related to weight gain and birth size parameters. BJOG: An International Journal of Obstetrics \& Gynaecology. 2006;113(11):1296-302.

133. Chin JR, Krause KM, Ostbye T, Chowdhury N, Lovelady CA, Swamy GK. Gestational weight gain in consecutive pregnancies. American journal of obstetrics and gynecology. 2010;203(3):279.e1-6.

134. Dipietro JA, Millet S, Costigan KA, Gurewitsch E, Caulfield LE. Psychosocial influences on weight gain attitudes and behaviors during pregnancy. Journal of the American Dietetic Association. 2003;103(10):1314-9.

135. Herring SJ, Oken E, Haines J, Rich-Edwards JW, Rifas-Shiman SL, Kleinman Sc DK, et al. Misperceived prepregnancy body weight status predicts excessive gestational weight gain: findings from a US cohort study. BMC Pregnancy Childbirth. 2008;8:54.

136. Gunderson EP, Rifas-Shiman SL, Oken E, Rich-Edwards JW, Kleinman KP, Taveras EM, et al. Association of Fewer Hours of Sleep at 6 Months Postpartum with Substantial Weight Retention at 1 Year Postpartum. American Journal of Epidemiology. 2008;167(2):178-87.

137. Kamysheva E, Skouteris H, Wertheim EH, Paxton SJ, Milgrom J. Examination of a multi-factorial model of body-related experiences during pregnancy: the relationships among physical symptoms, sleep quality, depression, self-esteem, and negative body attitudes. Body image. 2008;5(2):152-63.

138. Laraia B, Epel E, Siega-Riz AM. Food insecurity with past experience of restrained eating is a recipe for increased gestational weight gain. Appetite. 2013;65:178-84.

139. Laraia B, Messer L, Evenson K, Kaufman JS. Neighborhood factors associated with physical activity and adequacy of weight gain during pregnancy. Journal of urban health : bulletin of the New York Academy of Medicine. 2007 Nov;84(6):793-806.

140. Paul KH, Graham ML, Olson CM. The web of risk factors for excessive gestational weight gain in low income women. Matern Child Health J. 2013 Feb;17(2):344-51.

141. Phelan S, Phipps MG, Abrams B, Darroch F, Schaffner A, Wing RR. Practitioner advice and gestational weight gain. Journal of women's health (2002). 2011;20(4):585-91.

142. Drehmer M, Duncan BB, Kac G, Schmidt MI, Frasch MG. Association of Second and Third Trimester Weight Gain in Pregnancy with Maternal and Fetal Outcomes. PLoS ONE. 2013;8(1):e54704.

143. Stichting Perinatale Registratie Nederland. Grote Lijnen 1999-2012 [Perinatal care in the Netherlands 1999-2012]. Utrecht: PRN, 2013.

144. Quinlivan JA, Julania S, Lam L. Antenatal dietary interventions in obese pregnant women to restrict gestational weight gain to Institute of Medicine recommendations: a meta-analysis. Obstet Gynecol. 2011 Dec;118(6):1395-401.

145. Muktabhant B, Lumbiganon P, Ngamjarus C, Dowswell T. Interventions for preventing excessive weight gain during pregnancy. Cochrane Database Syst Rev. 2012;4:CD007145.

146. Brown A, Avery A. Healthy weight management during pregnancy: what advice and information is being provided. Journal of human nutrition and dietetics : the official journal of the British Dietetic Association. 2012;25(4):378-87.

147. Centraal Plan Bureau. Modal income definition: [Internet] 2015 [cited 6-5-2015] Available from: http://www.cpb.nl/en/node/. 
148. Brantsæter A, Haugen M, Rasmussen S, Alexander J, Samuelsen S, Meltzer H. Urine flavonoids and plasma carotenoids in the validation of fruit, vegetable and tea intake during pregnancy in the Norwegian Mother and Child Cohort Study (MoBa). Public Health Nutrition. 2007;10(8):838-47.

149. Peersman T. Portiegroottes [Portion sizes]. Waasmunster: Valetudo counseling; 2011.

150. Chasan-Taber L, Schmidt MD, Roberts DE, Hosmer D, Markenson G, Freedson PS. Development and validation of a Pregnancy Physical Activity Questionnaire. Med Sci Sports Exerc. 2004 Oct;36(10):1750-60.

151. Van Poppel M, Althuizen E, Van Mechelen W. Construct validity and responsiveness of the SQUASH for measuring physical activity in pregnancy. Journal of Science and Medicine in Sport. 2010;12(Supplement 2):e131. 152. Kolt G, Stephenson J, Robergs R, Thornton C. Validity of pedometers in measuring physical activity in pregnant women. Journal of Science and Medicine in Sport. 2010;12(Supplement 2):e154.

153. Bodnar L, Wisner K, Moses-Kolko E, Sit D, Hanusa B. Prepregnancy Body Mass Index, Gestational Weight Gain, and the Likelihood of Major Depressive Disorder During Pregnancy. Journal of Clinical Psychiatry. 2009;70(9): 1290-6.

154. Harris ST, Liu J, Wilcox S, Moran R, Gallagher A. Exercise during pregnancy and its association with gestational weight gain. Matern Child Health J. 2015 Mar;19(3):528-37.

155. Ryan RM, Deci EL. Self-determination theory and the facilitation of intrinsic motivation, social development, and well-being. The American psychologist. 2000;55(1):68-78.

156. Teixeira PJ, Carraça EV, Markland D, Silva MN, Ryan RM. Exercise, physical activity, and selfdetermination theory: a systematic review. The international journal of behavioral nutrition and physical activity. 2012;9:78.

157. Ajzen I. Residual Effects of Past on Later Behavior: Habituation and Reasoned Action Perspectives. Personality and Social Psychology Review. 2002;6(2):107-22.

158. Statline: CBS: Central Bureau of Statistics; 2014. Available from: https://www.cbs.nl/statweb.

159. Ferraro ZM, Gaudet L, Adamo KB. The potential impact of physical activity during pregnancy on maternal and neonatal outcomes. Obstet Gynecol Surv. 2012 Feb;67(2):99-110.

160. Ruchat S-M, Mottola MF. Preventing Long-Term Risk of Obesity for Two Generations: Prenatal Physical Activity Is Part of the Puzzle. Journal of Pregnancy. 2012;2012(2):1-33.

161. Schlaff RA, Holzman C, Maier KS, Pfeiffer KA, Pivarnik JM. Associations among gestational weight gain, physical activity, and pre-pregnancy body size with varying estimates of pre-pregnancy weight. Midwifery.

2014;30(11):1124-31.

162. Have MT, van der Heide A, Mackenbach JP, de Beaufort ID. An ethical framework for the prevention of overweight and obesity: a tool for thinking through a programme's ethical aspects. European journal of public health. 2013;23(2):299-305.

163. Tobias D, Bao W. Diet during Pregnancy and Gestational Weight Gain. Current Nutrition Reports. 2014;3(3):289-97.

164. Gaston A, Prapavessis H. Tired, moody and pregnant? Exercise may be the answer. Psychology \& health. 2013;28(12):1353-69.

165. Bodnar LM, Hutcheon JA, Platt RW, Himes KP, Simhan HN, Abrams B. Should Gestational Weight Gain Recommendations be Tailored by Maternal Characteristics? American Journal of Epidemiology. 2011;174(2):13646.

166. Gilson L, Doherty J, Loewenson R, Fransis V. Challenging inequity through health systems, final report Knowledge Networdk on Health Systems, final report Knowledge Network on Health Systems. WHO, 2007.

167. Audrain-McGovern J, Benowitz NL. Cigarette Smoking, Nicotine, and Body Weight. Clinical Pharmacology \& Therapeutics. 2011;90(1):164-8.

168. Knight K, Pressman E, Thornburg L. Decreased sleep duration in the third-trimester is not associated with excessive gestational weight gain. American Journal of Obstetrics and Gynecology. 2013;208(1):S60-S1.

169. Abeysena C, Jayawardana P. Sleep deprivation, physical activity and low income are risk factors for inadequate weight gain during pregnancy: A cohort study. Journal of Obstetrics and Gynaecology Research. 2011;37(7).

170. St-Onge MP. The role of sleep duration in the regulation of energy balance: effects on energy intakes and expenditure. Journal of clinical sleep medicine : JCSM : official publication of the American Academy of Sleep Medicine. 2013;9(1):73-80.

171. Bogaerts AF, Van den Bergh BR, Witters I, Devlieger R. Anxiety during early pregnancy predicts postpartum weight retention in obese mothers. Obesity (Silver Spring). 2013 Sep;21(9):1942-9.

172. Tanner-Smith EE, Steinka-Fry KT, Gesell SB. Comparative Effectiveness of Group and Individual Prenatal Care on Gestational Weight Gain. Maternal and child health journal. 2014;18(7):1711-20.

173. Buschur E, Kim C. Guidelines and interventions for obesity during pregnancy. Int J Gynaecol Obstet. 2012 Oct;119(1):6-10. 
174. Downs DS, Chasan Taber L, Evenson KR, Leiferman J, Yeo S. Physical activity and pregnancy: past and present evidence and future recommendations. Res Q Exerc Sport. 2012 Dec;83(4):485-502.

175. Melzer K, Schutz Y, Boulvain M, Kayser B. Physical activity and pregnancy: cardiovascular adaptations, recommendations and pregnancy outcomes. Sports Med. 2010 Jun 1;40(6):493-507.

176. Mudd LM, Owe KM, Mottola MF, Pivarnik JM. Health benefits of physical activity during pregnancy: an international perspective. Med Sci Sports Exerc. 2013 Feb;45(2):268-77.

177. Evenson KR, Barakat R, Brown WJ, Dargent-Molina P, Haruna M, Mikkelsen EM, et al. Guidelines for Physical Activity during Pregnancy: Comparisons From Around the World. American journal of lifestyle medicine. 2014 Mar;8(2):102-21.

178. Nederlands Instituut voor Sport en Bewegen. Nederlandse beweegnormen [Dutch norms for physical activity] [Internet] 2015 [cited 5-12-2016]. Available from: http://www.nisb.nl/weten/normen.html.

179. World health Organization. Global Strategy on Diet, Physical Activity and Health. Geneva: WHO, 2014.

180. Gaston A, Cramp A. Exercise during pregnancy: a review of patterns and determinants. J Sci Med Sport. 2011 Jul;14(4):299-305.

181. Cohen TR, Plourde H, Koski KG. Use of the Pregnancy Physical Activity Questionnaire (PPAQ) to identify behaviours associated with appropriate gestational weight gain during pregnancy. Journal of physical activity \& health. 2013 Sep;10(7):1000-7.

182. Poudevigne MS, O'Connor PJ. A review of physical activity patterns in pregnant women and their relationship to psychological health. Sports Med. 2006;36(1):19-38.

183. Weir Z, Bush J, Robson SC, McParlin C, Rankin J, Bell R. Physical activity in pregnancy: a qualitative study of the beliefs of overweight and obese pregnant women. BMC Pregnancy Childbirth. 2010;10:18.

184. Cannella D, Lobel M, Monheit A. Knowing is believing: information and attitudes towards physical activity during pregnancy. J Psychosom Obstet Gynaecol. 2010 Dec;31(4):236-42.

185. Johnson M, Campbell F, Messina J, Preston L, Buckley Woods H, Goyder E. Weight management during pregnancy: a systematic review of qualitative evidence. Midwifery. 2013 Dec;29(12):1287-96.

186. Da Costa D, Ireland K. Perceived benefits and barriers to leisure-time physical activity during pregnancy in previously inactive and active women. Women \& health. 2013;53(2):185-202.

187. Evenson KR, Bradley CB. Beliefs about exercise and physical activity among pregnant women. Patient education and counseling. 2010;79(1):124-9.

188. Merkx A, Ausems M, Bude L, de Vries R, Nieuwenhuijze MJ. Weight gain in healthy pregnant women in relation to pre-pregnancy BMI, diet and physical activity. Midwifery. 2015 Jul;31(7):693-701. (Chapter 3 of this thesis).

189. Norman P, Abraham C. Understanding and changing health behaviour : from health beliefs to selfregulation. Amsterdam [etc.] :: Harwood Academic Publishers; 2000.

190. Ajzen I. The Theory of Planned Behavior. Organizational Behavior and Human Decisio Proces. 1991;50:179-211.

191. Ajzen I. The theory of planned behaviour: reactions and reflections. Psychol Health. 2011

Sep;26(9):1113-27.

192. Weinstein ND, Sandman PM, Blalock S. The Precaution Adoption Proces Model. In: Glanz K, Rimer BK, Viswanath K, editors. Health behavior and health education: Theory, research and practice. 4th ed. San Francisco: Jossey-Bass; 2008. p. 123-65.

193. Clarke PE, Gross H. Women's behaviour, beliefs and information sources about physical exercise in pregnancy. Midwifery. 2004 Jun;20(2):133-41.

194. Thornton PL, Kieffer EC, Salabarria-Pena Y, Odoms-Young A, Willis SK, Kim H, et al. Weight, diet, and physical activity-related beliefs and practices among pregnant and postpartum Latino women: the role of social support. Matern Child Health J. 2006 Jan;10(1):95-104.

195. Connelly M, Brown H, van der Pligt $\mathrm{P}$, Teychenne M. Modifiable barriers to leisure-time physical activity during pregnancy: a qualitative study investigating first time mother's views and experiences. BMC Pregnancy Childbirth. 2015;15:100.

196. Leiferman J, Swibas T, Koiness K, Marshall JA, Dunn AL. My baby, my move: examination of perceived barriers and motivating factors related to antenatal physical activity. J Midwifery Womens Health. 2011 JanFeb;56(1):33-40.

197. Jukic A, Evenson K, Herring A, Wilcox A, Hartmann K, Daniels J. Correlates of physical activity at two time points during pregnancy. Journal of physical activity \& health. 2012 (9):325-35.

198. Haakstad LA, Bo K. Exercise in pregnant women and birth weight: a randomized controlled trial. BMC Pregnancy Childbirth. 2011;11:66.

199. Haakstad LA, Voldner N, Henriksen T, Bo K. Why do pregnant women stop exercising in the third trimester? Acta Obstet Gynecol Scand. 2009;88(11):1267-75. 
200. Owe KM, Nystad W, Bo K. Correlates of regular exercise during pregnancy: the Norwegian Mother and Child Cohort Study. Scandinavian journal of medicine \& science in sports. 2009 Oct;19(5):637-45.

201. Juhl M, Madsen M, Andersen AM, Andersen PK, Olsen J. Distribution and predictors of exercise habits among pregnant women in the Danish National Birth Cohort. Scandinavian journal of medicine \& science in sports. 2012 Feb;22(1):128-38.

202. Merkx A, Ausems M, Budé L, de Vries R, Nieuwenhuijze MJ. Dutch Midwives' Behaviour and Determinants in Promoting Healthy Gestational Weight Gain, Phase 2: A Quantitative Approach. International Journal of Childbirth. 2015;5(3):139-53. (Chapter 6 of this thesis).

203. Evenson KR, Pompeii LA. Obstetrician practice patterns and recommendations for physical activity during pregnancy. J Womens Health (Larchmt). 2010 Sep;19(9):1733-40.

204. Merkx A, Ausems M, Budé L, de Vries R, Nieuwenhuijze MJ. Dutch Midwives' Behaviour and Determinants in Promoting Healthy Gestational Weight Gain, Phase 1: A Qualitative Approach. International Journal of Childbirth. 2015;5(3):126-38. (Chapter 5 of this thesis).

205. Lindqvist M, Mogren I, Eurenius E, Edvardsson K, Persson M. "An on-going individual adjustment": a qualitative study of midwives' experiences counselling pregnant women on physical activity in Sweden. BMC Pregnancy Childbirth. 2014;14:343.

206. Fontein-Kuipers YJ, Bude L, Ausems M, de Vries R, Nieuwenhuijze MJ. Dutch midwives' behavioural intentions of antenatal management of maternal distress and factors influencing these intentions: an exploratory survey. Midwifery. 2014 Feb;30(2):234-41.

207. Currie S, Sinclair M, Murphy MH, Madden E, Dunwoody L, Liddle D. Reducing the decline in physical activity during pregnancy: a systematic review of behaviour change interventions. PLoS One. 2013;8(6):e66385. 208. Battle CL, Abrantes AM, Schofield CA, Kraines MA. Physical Activity as an Intervention for Antenatal Depression: Rationale for Developing Tailored Exercise Programs for Pregnant Women with Depression. J Midwifery Womens Health. 2015 Sep-Oct;60(5):479-82.

209. Seefat-van Teeffelen A, Nieuwenhuijze M, Korstjens I. Women want proactive psychosocial support from midwives during transition to motherhood: a qualitative study. Midwifery. 2011 Feb;27(1):e122-7.

210. Midwifery 2020 Programme. Midwifery 2020: Delivering Expectations. London: Midwifery $2020,2010$.

211. Mailey EL, McAuley E. Impact of a brief intervention on physical activity and social cognitive determinants among working mothers: a randomized trial. Journal of behavioral medicine. 2014 Apr;37(2):343-55. 212. Fieldwick D, Paterson H, Stephen M, Cameron A, Egan R, McFadden S, et al. Management of excess weight in pregnancy in Otago, New Zealand: a qualitative study with lead maternity carers. The New Zealand medical journal. 2014 Apr 11;127(1392):27-37.

213. Furness PJ, McSeveny K, Arden MA, Garland C, Dearden AM, Soltani H. Maternal obesity support services: a qualitative study of the perspectives of women and midwives. BMC Pregnancy Childbirth. 2011;11:69.

214. Willcox JC, Campbell KJ, van der Pligt P, Hoban E, Pidd D, Wilkinson S. Excess gestational weight gain: an exploration of midwives' views and practice. BMC Pregnancy Childbirth. 2012;12:102.

215. Brown MJ, Sinclair M, Liddle D, Hill AJ, Madden E, Stockdale J. A systematic review investigating healthy lifestyle interventions incorporating goal setting strategies for preventing excess gestational weight gain. PLoS One. 2012;7(7):e39503.

216. Thangaratinam S, Rogozinska E, Jolly K, Glinkowski S, Roseboom T, Tomlinson JW, et al. Effects of interventions in pregnancy on maternal weight and obstetric outcomes: meta-analysis of randomised evidence. BMJ (Clinical research ed). 2012;344:e2088.

217. Heslehurst N, Crowe L, Robalino S, Sniehotta FF, McColl E, Rankin J. Interventions to change maternity healthcare professionals' behaviours to promote weight-related support for obese pregnant women: a systematic review. Implement Sci. 2014;9:97.

218. de Geus E. Midwifery in the Netherlands Utrecht: Koninklijke Organisatie voor Verloskundigen (KNOV) [Royal Dutch organisation for midwives]; [Internet]. 2012 [cited 5-12-2016] Available from:

http://www.knov.nl/samenwerken/tekstpagina/489/midwifery-in-the-netherlands.

219. van Hassel D, Kasteleijn A, Kenens R. Cijfers uit de registratie van verloskundigen 2013 [Numbers of obstetric registration]. Utrecht: NIVEL, 2014.

220. KNOV. Opbouw van het verloskundig tarief in 2015 [Calculation for midwifery care in 2015] Utrecht: KNOV; 2015 [cited 20158 February]. Available from: www.knov.nl

221. Reitsma E, Groenen C, Fermie M. Takenpakket Eerstelijns Verloskunde 2007. Utrecht: KNOV, 2007.

222. de Boer J, Zeeman K, Offerhaus P. KNOV-standaard prenatale verloskundige begeleiding, wetenschappelijke onderbouweing (Guideline prenatal midwifery care). Utrecht: Koninklijke Nederlandse Organisatie Verloskundigen (KNOV) [Royal Dutch organisation for midwives]; 2008. 255 p.

223. Wiegers TA, Warmelink JC, Spelten ER, Klomp T, Hutton EK. Work and workload of Dutch primary care midwives in 2010. Midwifery. 2014 Sep;30(9):991-7. 
224. Nederlandse Vereniging voor Obstetrie \& Gynaecologie. Zwangerschap bij obesitas [Pregnancy in obese women]. Utrecht: NVOG 2009.

225. Gijsbers B, Mesters I, Knottnerus JA, Kester ADM, Schayck CPV. The Success of an Educational Program to Promote Exclusive Breastfeeding for 6 Months in Families with a History of Asthma: A Randomized Controlled Trial. pediatric asthma, allergy \& immunology. 2006;19(4):214-22.

226. Polit DF, Beck CT. Nursing research: generating and assessing evidence for nursing practice. Philadelphia, PA [etc]: Wolters Kluwer/Lippincott Williams \& Wilkins; 2012.

227. Heslehurst N, Russell S, McCormack S, Sedgewick G, Bell R, Rankin J. Midwives perspectives of their training and education requirements in maternal obesity: a qualitative study. Midwifery. $2013 \mathrm{Jul} ; 29(7): 736-44$.

228. Glanz K, Rimer BK, Viswanath K. Health behavior and health education : theory, research, and practice. 4th ed. ed. San Francisco, CA : Jossey-Bass; 2008.

229. Walsh D, Devane D. A metasynthesis of midwife-led care. Qual Health Res. 2012 Jul;22(7):897-910.

230. Olsson P, Sandman PO, Jansson L. Antenatal 'booking' interviews at midwifery clinics in Sweden: a qualitative analysis of five video-recorded interviews. Midwifery. 1996 Jun;12(2):62-72.

231. Noordman J, van der Weijden T, van Dulmen S. Communication-related behavior change techniques used in face-to-face lifestyle interventions in primary care: A systematic review of the literature. Patient Education and Counseling. 2012;89(2):227-44.

232. World Health Organization. Milestones in Health Promotion, Statements form Global Conferences. WHO, 1986.

233. Padilla JL, Benitez I, Castillo M. Obtaining validity evidence by cognitive interviewing to interpret psychometric results. Methodology. 2013;9(3):113-22.

234. Field A. Discovering statistics using SPSS (and sex and drugs and rock ' $n$ ' roll). 3 ed. Los Angelas, CA [etc]: Sage; 2009.

235. Stotland NE, Gilbert P, Bogetz A, Harper CC, Abrams B, Gerbert B. Preventing excessive weight gain in pregnancy: how do prenatal care providers approach counseling? J Womens Health (Larchmt). 2010 Apr;19(4):80714.

236. McDonald SDMD, Pullenayegum EP, Taylor VHMDP, Lutsiv O, Bracken KMD, Good CRNNP, et al. Despite 2009 guidelines, few women report being counseled correctly about weight gain during pregnancy. American Journal of Obstetrics and Gynecology. 2011;205(4):333.e1-.e6.

237. Wilkinson S, Poad D, Stapleton H. Maternal overweight and obesity: a survey of clinicians' characteristics and attitudes, and their responses to their pregnant clients. BMC Pregnancy and Childbirth [Internet]. 2013; 13(1):[1-8 pp.].

238. Ferrari ReM, Siega-Riz AM. Provider Advice About Pregnancy Weight Gain and Adequacy of Weight Gain. Maternal and child health journal. 2013;17(2):256-64.

239. Jamar J. Vroedvrouwenschool, 100 jaar Moederschapszorg in Limburg [Midwifery School, 100 years of motherhoodcare in Limburg]. Hilversum: Verloren; 2009.

240. Voorn M. De informatiebehoefte over een gezonde leefstijl van (aanstaande) ouders van jonge kinderen [The needs in information about a healthy lifestyle of parents of young and unborn children]. Amsterdam:

Ministerie van Volksgezondheid, welzijn en sport, 2013.

241. Hingstman L, Van Hassel D, Kenens R. Cijfers uit de registratie van verloskundigen 2012 [Numbers of obstetric registration]. Utrecht: NIVEL, 2013.

242. Furber CM, McGowan L, Bower P, Kontopantelis E, Quenby S, Lavender T. Antenatal interventions for reducing weight in obese women for improving pregnancy outcome. Cochrane Database Syst Rev.

2013;1:CD009334.

243. Hill B, Skouteris H, Fuller-Tyszkiewicz M. Interventions designed to limit gestational weight gain: a systematic review of theory and meta-analysis of intervention components. Obes Rev. 2013 Jun;14(6):435-50. 244. Thangaratinam S, Rogozinska E, Jolly K, Glinkowski S, Duda W, Borowiack E, et al. Interventions to reduce or prevent obesity in pregnant women: a systematic review. Health technology assessment (Winchester, England). 2012 Jul;16(31):iii-iv, 1-191.

245. Homer CS, Friberg IK, Dias MA, ten Hoope-Bender P, Sandall J, Speciale AM, et al. The projected effect of scaling up midwifery. Lancet (London, England). 2014;384(9948):1146-57.

246. Renfrew MJ, McFadden A, Bastos MH, Campbell J, Channon AA, Cheung NF, et al. Midwifery and quality care: findings from a new evidence-informed framework for maternal and newborn care. Lancet (London, England). 2014;384(9948):1129-45.

247. ten Hoope-Bender PMBA, de Bernis LMD, Campbell JMPH, Downe SP, Fauveau VP, Fogstad HMHA, et al. Improvement of maternal and newborn health through midwifery. The Lancet. 2014;384(9949):1226-35. 
248. Van Lerberghe W, Matthews Z, Achadi E, Ancona C, Campbell J, Channon A, et al. Country experience with strengthening of health systems and deployment of midwives in countries with high maternal mortality. Lancet (London, England). 2014;384(9949):1215-25.

249. Brug J, Oenema A, Ferreira I. Theory, evidence and Intervention Mapping to improve behavior nutrition and physical activity interventions. Int J Behav Nutr Phys Act. 2005 Apr 4;2(1):2.

250. Noar SM, Crosby R, Benac C, Snow G, Troutman A. Application of the attitude-social influence-efficacy model to condom use among African-American STD clinic patients: implications for tailored health communication. AIDS and behavior. $2011 \mathrm{Jul}$;15(5):1045-57.

251. Temel S, Birnie E, Sonneveld HM, Voorham AJ, Bonsel GJ, Steegers EA, et al. Determinants of the intention of preconception care use: lessons from a multi-ethnic urban population in the Netherlands. International journal of public health. 2013 Apr;58(2):295-304.

252. Steckler A, Linnan L, editors. Process evaluation for public health interventionsand research. San Francisco: Jossey-Bass; 2002.

253. Zamora-Kapoor A, Walker L. Excessive Gestational Weight Gain in Adolescent and Adult Pregnancies: An Overlooked Public Health Problem. The Journal of Pediatrics. 2015;167( 3):515-7.

254. Phelan S, Jankovitz K, Hagobian T, Abrams B. Reducing excessive gestational weight gain: lessons from the weight control literature and avenues for future research. Women's health (London, England). 2011 Nov;7(6):641-61.

255. Streuling I, Beyerlein A, von Kries R. Can gestational weight gain be modified by increasing physical activity and diet counseling? A meta-analysis of interventional trials. Am J Clin Nutr. 2010 Oct;92(4):678-87.

256. Olander EK, Atkinson L, Edmunds JK, French DP. The views of pre- and post-natal women and health professionals regarding gestational weight gain: An exploratory study. Sexual \& Reproductive Healthcare. 2011 $1 / / ; 2(1): 43-8$.

257. Stengel MR, Kraschnewski JL, Hwang SW, Kjerulff KH, Chuang $\mathrm{CH}$. "What my doctor didn't tell me": examining health care provider advice to overweight and obese pregnant women on gestational weight gain and physical activity. Womens Health Issues. 2012 Nov-Dec;22(6):e535-40.

258. De Vries H, Dijkstra M, Kuhlman P, . Self-efficacy: the third factor besides attitude and subjective norm as a predictor of behavioural intentions. Health Education Research. 1988;3(3):273-82.

259. Merkx A, Interventies rondom bevorderen van een gezonde gewichtstoename tijdens de zwangerschap op een rij [Interventions to promote healthy gestational weight gain at a glance]. Nederlands Congres Volksgezondheid 2012; posterpresentatie; Amsterdam.

260. Szwajcer E, Hiddink G, Maas L, Koelen M, Woerkum van G. Nutrition awareness before and throughout different trimesters in pregnancy: a quantitative study among Dutch women. Family Practice i82-i88. 2012;1(suppl 1):i82-i8.

261. Groth SW, Kearney MH. Diverse women's beliefs about weight gain in pregnancy. J Midwifery Womens Health. 2009 Nov-Dec;54(6):452-7.

262. Herring SJ, Henry TQ, Klotz AA, Foster GD, Whitaker RC. Perceptions of low-income African-American mothers about excessive gestational weight gain. Matern Child Health J. 2012 Dec;16(9):1837-43.

263. Herring SJ, Rose MZ, Skouteris H, Oken E. Optimizing weight gain in pregnancy to prevent obesity in women and children. Diabetes Obes Metab. 2012 Mar;14(3):195-203.

264. Palmer JL, Jennings GE, Massey L. Development of an assessment form: attitude toward weight gain during pregnancy. J Am Diet Assoc. 1985 Aug;85(8):946-9.

265. Tovar A, Chasan-Taber L, Bermudez OI, Hyatt RR, Must A. Knowledge, attitudes, and beliefs regarding weight gain during pregnancy among Hispanic women. Matern Child Health J. 2010 Nov;14(6):938-49.

266. Cogswell ME, Scanlon KS, Fein SB, Schieve LA. Medically advised, mother's personal target, and actual weight gain during pregnancy. Obstet Gynecol. 1999 Oct;94(4):616-22.

267. Stotland N, Tsoh JY, Gerbert B. Prenatal weight gain: who is counseled? J Womens Health (Larchmt). 2012 Jun;21(6):695-701.

268. Stotland NE, Haas JS, Brawarsky P, Jackson RA, Fuentes-Afflick E, Escobar GJ. Body mass index, provider advice, and target gestational weight gain. Obstet Gynecol. 2005 Mar;105(3):633-8.

269. Cramp AG, Bray SR. A prospective examination of exercise and barrier self-efficacy to engage in leisuretime physical activity during pregnancy. Ann Behav Med. 2009 Jun;37(3):325-34.

270. Jans SM, de Jonge A, Henneman L, Cornel MC, Lagro-Janssen AL. Attitudes of general practitioners and midwives towards ethnicity-based haemoglobinopathy-carrier screening. Eur J Hum Genet. 2012 Nov;20(11):11127.

271. Prochaska J, Redding C, Evers K. The Transtheoretical Model and Stages of Change. In: Glanz K, Rimer B, Viswanath K, editors. Health behavior and health education: Theory, Research and Practice. 4th ed. San Francisco: Jossey-Bass; 2008. p. 97-121. 
272. Bandura A. Social foundations of thought and action: A social cognitive theory. Englewood Cliffs, NJ: Prentice- Hall, Inc; 1986.

273. Locke E, Latham G. A theory of goal setting and task performance. Englewood Cliffs: Prentice Hall; 1990.

274. Lustria ML, Cortese J, Noar SM, Glueckauf RL. Computer-tailored health interventions delivered over the Web: review and analysis of key components. Patient Educ Couns. 2009 Feb;74(2):156-73.

275. Petty R, Caccioppo J. Communication and persuasion: Central and peripheral routes to attitude change. NewYork: Springer-Verag; 1986.

276. Bennett GG, Glasgow RE. The delivery of public health interventions via the Internet: actualizing their potential. Annual review of public health. 2009;30:273-92.

277. Webb TL, Joseph J, Yardley L, Michie S. Using the internet to promote health behavior change: a systematic review and meta-analysis of the impact of theoretical basis, use of behavior change techniques, and mode of delivery on efficacy. Journal of medical Internet research. 2010;12(1):e4.

278. World Health Organization. Nurses and midwives: A force for health. WHO European Strategy for Continuing Education for Nurses and Midwives. Denmark: Copenhagen: WHO, 2003.

279. Kwak L, Kremers SP, Werkman A, Visscher TL, van Baak MA, Brug J. The NHF-NRG In Balance-project: the application of Intervention Mapping in the development, implementation and evaluation of weight gain prevention at the worksite. Obes Rev. $2007 \mathrm{Jul} ; 8(4): 347-61$.

280. Rogers E. Diffusion of innovations. 4 ed. New York: The Free Press.; 1995.

281. Heslehurst N, Newham J, Maniatopoulos G, Fleetwood C, Robalino S, Rankin J. Implementation of pregnancy weight management and obesity guidelines: A meta-synthesis of healthcare professionals' barriers and facilitators using the Theoretical Domains Framework. Pregnancy Hypertens. 2014 Jul;4(3):234-5.

282. Merkx A, Ausems M, de Vries R, Nieuwenhuijze M. "Come On!" Using Intervention Mapping to help healthy pregnant women achieve healthy weight gain. Public Health Nutrition 2017; 1-15 doi:10.1017/S1368980017000271. . (Chapter 7 of this thesis)

283. Brantsaeter AL, Haugen M, Rasmussen SE, Alexander J, Samuelsen SO, Meltzer HM. Urine flavonoids and plasma carotenoids in the validation of fruit, vegetable and tea intake during pregnancy in the Norwegian Mother and Child Cohort Study (MoBa). Public health nutrition. 2007;10(8):838-47.

284. Field A. Discovering statistics using SPSS. 3 ed. London, California, New Delhi, Singapore: Sage Publications; 2009.

285. Althuizen E, van der Wijden CL, van Mechelen W, Seidell JC, van Poppel MN. The effect of a counselling intervention on weight changes during and after pregnancy: a randomised trial. BJOG. 2013 Jan;120(1):92-9.

286. O'Brien OA, McCarthy M, Gibney ER, McAuliffe FM. Technology-supported dietary and lifestyle interventions in healthy pregnant women: a systematic review. Eur J Clin Nutr. 2014 Jul;68(7):760-6.

287. Spijkerman MP, Pots WT, Bohlmeijer ET. Effectiveness of online mindfulness-based interventions in improving mental health: A review and meta-analysis of randomised controlled trials. Clin Psychol Rev. $2016 \mathrm{Apr}$ 1;45:102-14.

288. Hutchesson MJ, Rollo ME, Krukowski R, Ells L, Harvey J, Morgan PJ, et al. eHealth interventions for the prevention and treatment of overweight and obesity in adults: a systematic review with meta-analysis. Obes Rev. 2015 May;16(5):376-92.

289. Fontein Kuipers YJ, Ausems M, de Vries R, Nieuwenhuijze MJ. The effect of Wazzup Mama?! An antenatal intervention to prevent or reduce maternal distress in pregnancy. Arch Womens Ment Health. 2016 Oct;19(5):779-88.

290. Bayer O, Ensenauer R, Nehring I, von Kries R. Effects of trimester-specific and total gestational weight gain on children's anthropometrics. BMC Pregnancy Childbirth. 2014;14:351.

291. Berenson AB, Pohlmeier AM, Laz TH, Rahman M, Saade G. Obesity Risk Knowledge, Weight Misperception, and Diet and Health-Related Attitudes among Women Intending to Become Pregnant. J Acad Nutr Diet. 2016 Jan;116(1):69-75.

292. Tielemans MJ, Garcia AH, Peralta Santos A, Bramer WM, Luksa N, Luvizotto MJ, et al. Macronutrient composition and gestational weight gain: a systematic review. Am J Clin Nutr. 2016 Jan;103(1):83-99.

293. Tian C, Hu C, He X, Zhu M, Qin F, Liu Y, et al. Excessive weight gain during pregnancy and risk of macrosomia: a meta-analysis. Arch Gynecol Obstet. 2016 Jan;293(1):29-35.

294. Aşcı Ö, Rathfisch G. Effect of lifestyle interventions of pregnant women on their dietary habits, lifestyle behaviors, and weight gain: a randomized controlled trial. Journal of Health, Population and Nutrition. 2016;35(1). 295. Baas Cl, Erwich JJ, Wiegers TA, de Cock TP, Hutton EK. Women's Suggestions for Improving Midwifery Care in The Netherlands. Birth. 2015 Dec;42(4):369-78.

296. O'Malley-Keighran MP, Lohan G. Encourages and guides, or diagnoses and monitors: Woman centredness in the discourse of professional midwifery bodies. Midwifery 2016;43(2016);48-58 
297. Twinomujuni C, Nuwaha F, Babirye JN Understanding the Low Level of Cervical Cancer Screening in Masaka Uganda Using the ASE Model: A Community-Based Survey. 2015 PLoS ONE 10(6): e0128498.

298. De Vries H, Mudde A, Leijs I, Charlton A, Vartiainen E, Buijs G, Clemente MP, Storm H, González Navarro A, Prins T, Kremers S. The European Smoking Prevention Framework Approach (ESFA): an example of Integral Prevention; Health Educ Res, 18 (2003), pp. 611-626

299. van der Wulp, N.Y., Hoving, C. \& de Vries, H. Partner's Influences and Other Correlates of Prenatal Alcohol Use; Matern Child Health J. 2015 Apr;19(4):908-16.

300. De Vries H, Kremers S, Smeets T, Brug J, Eijmael K. The effectiveness of tailored feedback and action plans in an intervention addressing multiple health behaviors. American Journal of Health Promotion (2008). 22 (6): 417-425.

301. Reinwand DA, Crutzen R, Storm V, Wienert J, Kuhlmann T, de Vries H, Lippke S. Generating and predicting high quality action plans to facilitate physical activity and fruit and vegetable consumption: results from an experimental arm of a randomised controlled trial. BMC Public Health. 2016 Apr 12;16:317

302. Naidoo S, Satorius BK, de Vries H, Taylor M. Verbal Bullying Changes Among Students Following an Educational Intervention Using the Integrated Model for Behavior Change. J Sch Health. 2016 Nov;86(11):813-822

303. Goebbels AF, Nicholson JM, Walsh K, de Vries H. Teachers' reporting of suspected child abuse and neglect: behaviour and determinants. Health Educ Res. 2008 Dec;23(6):941-51

304. Stuurgroep Zwangerschap en Geboorte. Een goed begin. Veilige zorg rond zwangerschap en geboorte [Starting safe, good care in pregnancy and childbirth]. Minsterie van Volksgezondheid, welzijn en sport, 2009.

305. Overcash RT, Hull AD, Moore TR, LaCoursiere DY. Early Second Trimester Weight Gain in Obese Women Predicts Excessive Gestational Weight Gain in Pregnancy. Maternal and child health journal. 2015;19(11):2412-8.

306. Sachs JD, McArthur JW. The millennium project: a plan for meeting the millennium development goals. The Lancet. 2005;365(9456):347-53.

307. Nieuwenhuijze M. The art and science of midwifery. 25 May. Maastricht: Inaugurale rede Lectoraat Midwifery Science; 2016.

308. Michie S, Atkins L, West R. The behaviour change wheel: a guide to designing interventions. Needed: physician leaders. 2014:26.

309. Van der Pas A. A serious case of neglect: the parental experience of child rearing: outline for a psychological theory of painting: Eburon Delft; 2003.

310. Hoek M. Ontheemd ouderschap. Amsterdam: Universiteit van Amsterdam; 2008.

311. Conner M, Norman P. Predicting health behaviour: McGraw-Hill Education (UK); 2005.

312. World Health Organisation. Endocrine disrupters and child health, Possible developmental early effects of endocrine disrupters on child health. World Health Organisation, 2012.

313. Fisher M, Arbuckle TE, Liang CL, LeBlanc A, Gaudreau E, Foster WG, et al. Concentrations of persistent organic pollutants in maternal and cord blood from the maternal-infant research on environmental chemicals (MIREC) cohort study. Environmental health : a global access science source. 2016;15(1):59.

314. Bazer FW, Wu G, Johnson GA, Wang X. Environmental factors affecting pregnancy: endocrine disrupters, nutrients and metabolic pathways. Molecular and cellular endocrinology. 2014 Dec;398(1-2):53-68.

315. Phillips KP, Foster WG. Key developments in endocrine disrupter research and human health. Journal of toxicology and environmental health Part B, Critical reviews. 2008 Mar;11(3-4):322-44.

316. Sharma E, Mustafa M, Pathak R, Guleria K, Ahmed RS, Vaid NB, et al. A case control study of gene environmental interaction in fetal growth restriction with special reference to organochlorine pesticides. European journal of obstetrics, gynecology, and reproductive biology. 2012 Apr;161(2):163-9.

317. Ashley-Martin J, Dodds L, Arbuckle TE, Morisset AS, Fisher M, Bouchard MF, et al. Maternal and Neonatal Levels of Perfluoroalkyl Substances in Relation to Gestational Weight Gain. Int J Environ Res Public Health. 2016 Jan;13(1).

318. Lindsay KL, Walsh CA, Brennan L, McAuliffe FM. Probiotics in pregnancy and maternal outcomes: a systematic review. J Matern Fetal Neonatal Med. 2013 May;26(8):772-8.

319. Guarner F, Malagelada JR. Gut flora in health and disease. Lancet. 2003 Feb 8;361(9356):512-9.

320. Ley RE. Obesity and the human microbiome. Current opinion in gastroenterology. 2010 Jan;26(1):5-11.

321. Sanz Y, Santacruz A, Gauffin P. Gut microbiota in obesity and metabolic disorders. Proc Nutr Soc. 2010 Aug;69(3):434-41.

322. Dunlop AL, Mulle JG, Ferranti EP, Edwards S, Dunn AB, Corwin EJ. Maternal Microbiome and Pregnancy Outcomes That Impact Infant Health: A Review. Advances in neonatal care : official journal of the National Association of Neonatal Nurses. 2015 Dec;15(6):377-85.

323. Fouelifack FY, Fouedjio JH, Fouogue JT, Sando Z, Fouelifa LD, Mbu RE. Associations of body mass index and gestational weight gain with term pregnancy outcomes in urban Cameroon: a retrospective cohort study in a tertiary hospital. BMC research notes. 2015 Dec 19;8:806. 
324. Haugen M, Brantsaeter AL, Winkvist A, Lissner L, Alexander J, Oftedal B, et al. Associations of prepregnancy body mass index and gestational weight gain with pregnancy outcome and postpartum weight retention: a prospective observational cohort study. BMC Pregnancy Childbirth. 2014 Jun 11;14:201.

325. Asvanarunat E. Outcomes of gestational weight gain outside the Institute of Medicine Guidelines. J Med Assoc Thai. 2014 Nov;97(11):1119-25.

326. Park S, Sappenfield WM, Bish C, Salihu H, Goodman D, Bensyl DM. Assessment of the Institute of Medicine recommendations for weight gain during pregnancy: Florida, 2004-2007. Matern Child Health J. 2011 Apr;15(3):289-301.

327. Li C, Liu Y, Zhang W. Joint and Independent Associations of Gestational Weight Gain and Pre-Pregnancy Body Mass Index with Outcomes of Pregnancy in Chinese Women: A Retrospective Cohort Study. PLoS One. 2015;10(8):e0136850.

328. Daemers DO, Wijnen HA, van Limbeek EB, Budé LM, Nieuwenhuijze MJ, Spaanderman ME, et al. The effect of gestational weight gain on likelihood of referral to obstetric care for women eligible for primary, midwifeled care after antenatal booking. Midwifery. 2016;34:123-32.

329. Savitz DA, Stein CR, Siega-Riz AM, Herring AH. Gestational weight gain and birth outcome in relation to prepregnancy body mass index and ethnicity. Annals of epidemiology. 2011;21(2):78-85.

330. Cedergren MI. Optimal gestational weight gain for body mass index categories. Obstet Gynecol. 2007 Oct;110(4):759-64.

331. Bogaerts A, Ameye L, Martens E, Devlieger R. Weight loss in obese pregnant women and risk for adverse perinatal outcomes. Obstet Gynecol. 2015 Mar;125(3):566-75.

332. Faucher MA, Barger MK. Gestational weight gain in obese women by class of obesity and select maternal/newborn outcomes: a systematic review. Women and Birth. 2015;28(3):e70-e9.

333. Kapadia MZ, Park CK, Beyene J, Giglia L, Maxwell C, McDonald SD. Can we safely recommend gestational weight gain below the 2009 guidelines in obese women? A systematic review and meta-analysis. Obes Rev. 2015 Mar;16(3):189-206.

334. Hutcheon JA, Platt RW, Abrams B, Himes KP, Simhan HN, Bodnar LM. Pregnancy weight gain charts for obese and overweight women. Obesity. 2015;23(3):532-5.

335. Bodnar LM, Hutcheon JA, Parisi SM, Pugh SJ, Abrams B. Comparison of Gestational Weight Gain z-Scores and Traditional Weight Gain Measures in Relation to Perinatal Outcomes. Paediatric and perinatal epidemiology. 2015;29(1):11-21.

336. Nieuwenhuijze MJ. On speaking terms: choice and shared decision-making in maternity care. Nijmegen: Radboud Universiteit Nijmegen; 2014.

337. Miller WR, Rollnick S. Motivational interviewing: Helping people change: Guilford press; 2012.

338. Ruifrok AE, Rogozinska E, van Poppel MNM, Rayanagoudar G, Kerry S, de Groot CJM, et al. Study protocol: differential effects of diet and physical activity based interventions in pregnancy on maternal and fetal outcomes-individual patient data (IPD) meta-analysis and health economic evaluation. Systematic Reviews. 2014;3(1).

339. Daemers DOA, Van Limbeek EBM, Budé LM, Wijnen HAA, Nieuwenhuijze MJ, De Vries RG. The use of midwife-led primary prenatal care by obese women in the Netherlands: an explorative cohort study. Midwifery. http://dx.doi.org/10.1016/j.midw.2016.11.002. 


\section{Abbreviations}

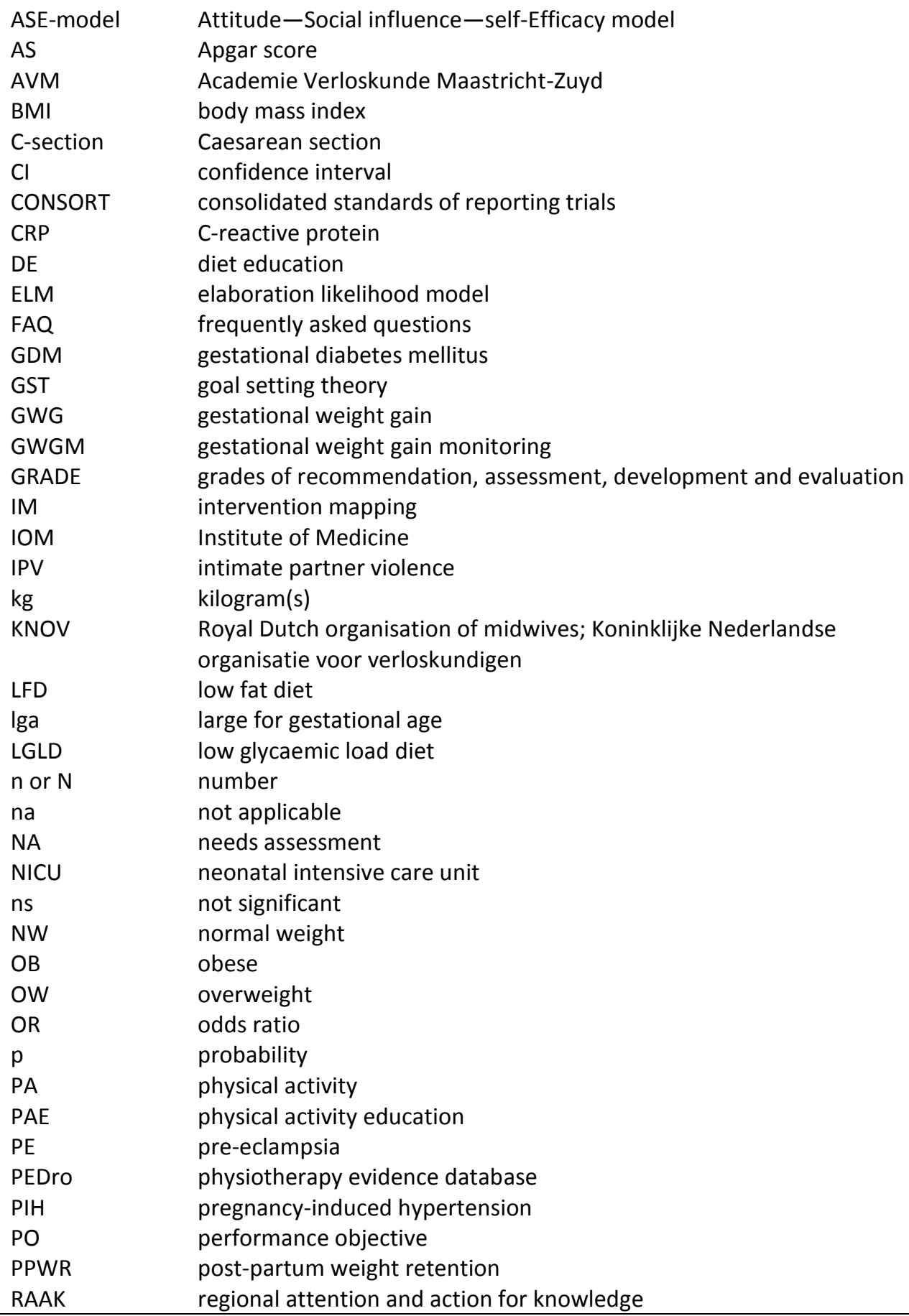


RCT

REVman

$\mathrm{RR}$

SCT

$\mathrm{sd}$

se

SE

SES

sga

TTM

USA

UW

WHO

WIC

randomized controlled trial

review manager

relative risk

social cognitive theory

standard deviation

self-efficacy

standard error

social economic status

small for gestational age

trans theoretical model

United States of America

underweight

World Health Organisation

women, infants and children program 


\section{Samenvatting}

Zwangerschap en de geboorte van een kind zijn belangrijke levensgebeurtenissen, die impact hebben op de emotionele en de fysieke gezondheid. Tijdens de zwangerschap verandert het vrouwenlichaam zichtbaar door het groter worden van de buik. De gewichtstoename die daarbij hoort is het onderwerp van dit proefschrift. We (de onderzoeksgroep waar de auteur toe behoort) beschrijven de ontwikkeling en de evaluatie van een interventie die erop gericht is om meer vrouwen een gezonde gewichtstoename tijdens de zwangerschap (GTZ) te laten verkrijgen.

\section{Hoofdstuk 1}

\section{Algemene introductie}

In het eerste hoofdstuk wordt de relevantie van het onderwerp beschreven, wordt de vraag die centraal staat verwoord en wordt weergegeven hoe het proefschrift is opgebouwd. Nederlandse verloskundigen die in de eerste lijn werken, lieten weten dat ze behoefte hadden aan een interventie om het probleem van een te hoge GTZ aan te pakken. Een gezonde GTZ wordt gedefinieerd als een gewichtstoename binnen de richtlijnen die zijn opgesteld door het Amerikaanse Institute of Medicine (IOM). Een te hoge GTZ komt vaak voor en is geassocieerd met meerdere ongezonde uitkomsten, zoals overgewicht op de lange termijn voor zowel moeder als kind. Maar ook een te lage GTZ komt regelmatig voor en dient vermeden te worden, omdat ook daaraan negatieve gevolgen gekoppeld worden, met name een te laag geboortegewicht en vroeggeboorte. Er is redelijk wat onderzoek gedaan naar de gewichtstoename van subgroepen; bijvoorbeeld vrouwen met een verhoogde kans op diabetes gravidarum ("zwangerschapsuiker"), of vrouwen met een hoge Body Mass Index (BMI). In dit proefschrift is gekozen om de GTZ van gezonde zwangere vrouwen te bestuderen, om daarmee bij te dragen aan de algemene preventie van complicaties die te maken hebben met een ongezonde GTZ.

Dit proefschrift heeft als centrale vraag: Kunnen we een wetenschappelijk goed onderbouwd programma ontwikkelen om Nederlandse verloskundigen te helpen met het effectief bevorderen van een gezonde GTZ? Om deze vraag te beantwoorden zijn de volgende sub vragen geformuleerd:

- Welke wetenschappelijk goed onderbouwde interventies bestaan er al om een gezonde GTZ te bevorderen en wat kunnen we daarvan leren? (hoofdstuk 2)

- Welke persoonlijke kenmerken en gedragsfactoren van zwangere vrouwen hangen samen met een gezonde GTZ? (hoofdstuk 3 en 4)

- Wat doen Nederlandse verloskundigen op dit moment om een gezonde GTZ te bevorderen? (hoofdstuk 5 en 6 )

- Welke systematisch ontwikkelde prenatale interventie zal een gezonde GTZ binnen de verloskundige zorg bevorderen? (hoofdstuk 7) 
- Is de ontwikkelde interventie effectief in het bevorderen van een gezonde GTZ? (hoofdstuk 8)

Het Intervention Mapping (IM) Protocol van Bartholomew et al. werd gebruikt om de interventie te ontwikkelen. Dit protocol bestaat uit zes stappen, weergegeven in Figuur 1. Stap 1 is de probleem analyse, stap 2 is het formuleren van programmadoelen. In stap 3 worden theoretische interventie methoden en praktische strategieën gekozen om de doelen te kunnen bereiken, waarna in stap 4 de programma onderdelen en materialen ontwikkeld worden. In stap 5 wordt een implementatieplan voor de interventie ontwikkeld en in de laatste stap wordt een evaluatie plan ontwikkeld. Het IM protocol is een iteratief proces; dat wil zeggen dat het steeds kan worden bijgesteld op basis van nieuwe bevindingen. Het kan nodig zijn om dan weer terug te gaan naar een stap die al gezet is.

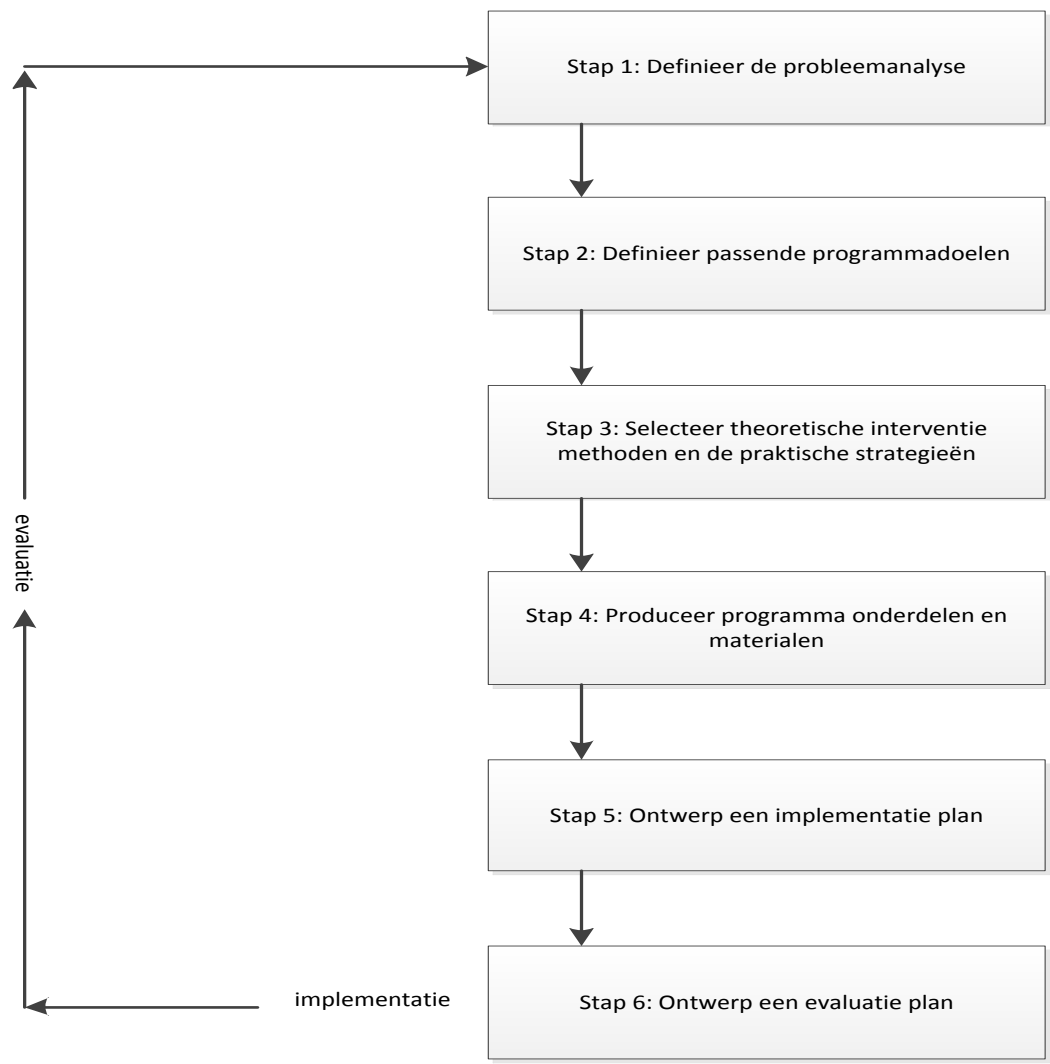

Figuur 1 Intervention Mapping Process (bron Bartholomew et al. 2011)

\section{Hoofdstuk 2}

Interventies gericht op het bereiken van een gezonde gewichtstoename tijdens de zwangerschap: Een systematische review / meta-analyse

Hoofdstuk twee beschrijft een uitgebreide review van de literatuur over bestaande interventies die gericht zijn op het bereiken van een gezonde GTZ. Daartoe werden 
relevante databases, tijdschriften en referentielijsten systematisch doorzocht op zowel gerandomiseerde als niet gerandomiseerde interventies die als primaire uitkomstmaat GTZ hadden en na 2001 waren gepubliceerd (laatste search op 30 september 2011). Er werden 28 studies gevonden, met in totaal 7410 participanten. Na zorgvuldige kwaliteitscontrole bleven er 14 gerandomiseerde studies en één niet gerandomiseerde studie over waarvan de resultaten werden gebruikt. De meeste studies kwamen uit de Verenigde Staten van Amerika en Europa. De helft van de interventies focuste op een enkel thema (dieet, beweging of monitoren van gewicht), de andere helft combineerde twee of drie van deze thema's. Veel interventies waren voor specifieke doelgroepen zoals tienermoeders of vrouwen met een verhoogde kans op diabetes gravidarum. Slechts een paar interventies richtte zich op gezonde zwangere vrouwen. Op drie groepen werden meta-analyses verricht: "dieet voor obese vrouwen"; "beweegprogramma voor vrouwen uit alle BMI-groepen" en "gecombineerde interventie voor vrouwen uit alle BMI-groepen". Uit de meta-analyse bleek dat interventies met een dieet voor obese vrouwen gemiddeld leidde tot $8,41 \mathrm{~kg}$ minder GTZ $(95 \% \mathrm{Cl}-10.49,-6.34 \mathrm{~kg}$ ), een beweegprogramma voor vrouwen uit alle BMI-groepen resulteerde in gemiddeld $0.83 \mathrm{~kg}$ minder $\mathrm{GTZ}$ ( $95 \% \mathrm{Cl}-1.47$, $0.19 \mathrm{~kg}$ ) en de drie gecombineerde interventie studies bleken in de meta-analyse geen significant effect te laten zien. Geen van de onderzochte studies had gemeten in hoeverre de GTZ binnen de IOM richtlijnen viel. Secundaire uitkomstmaten gaven inconsistente resultaten.

We concludeerden dat een goed werkende interventie voor gezonde zwangere vrouwen nog niet bestond. De totale GTZ voor obese vrouwen kan tot ruim acht kilogram verminderd worden door het volgen van een dieet met begeleiding. In mindere mate geeft beweging voor vrouwen uit alle BMI-groepen minder GTZ.

\section{Hoofdstuk 3}

\section{De gewichtstoename van zwangere vrouwen in relatie tot hun body mass index voor de zwangerschap, voeding en beweging.}

De studie, beschreven in hoofdstuk drie richt zich op de relatie tussen GTZ van gezonde zwangere vrouwen en de IOM-richtlijnen, de BMI voor de zwangerschap, de voeding en het beweegpatroon.

In totaal vulden 455 gezonde zwangere vrouwen die onder controle waren bij een verloskundige in de eerste lijn een uitgebreide vragenlijst in die voor deze gelegenheid was ontworpen. Er werd geen voorwaarde aan de zwangerschapsduur gesteld. Vrouwen vulden hun lichaamsgewicht voorafgaand aan de zwangerschap in en vulden ook in hoe zwaar ze waren op het moment van invullen van de vragenlijst. De GTZ werd berekend aan de hand van de IOM-richtlijnen en het resultaat werd ingedeeld in drie categorieën: conform de richtlijnen, erboven of eronder. Overige vragen gingen over persoonlijke karakteristieken, eetgedrag en beweeggedrag. Ook werden vragen voorgelegd over attitude, sociale invloed 
en eigen effectiviteit; (ASE)-determinanten van de gedragingen met betrekking tot monitoren van de GTZ, bewegen en voedingsinname.

Een multinomiale regressie analyse werd uitgevoerd, waarbij de GTZ ten opzichte van de IOM-richtlijnen als uitkomstmaat werd gehanteerd (conform de richtlijn als referentie) en als determinanten de BMI (van voor de zwangerschap), voeding (hoeveelheid geconsumeerde groente, voldoen aan de vis norm en voldoen aan de fruit norm) en beweging (motivatie om te bewegen, beweging voor de zwangerschap en verminderen van bewegen in de zwangerschap). Daarbij werd gecorrigeerd voor leeftijd, duur van de zwangerschap, pariteit, etniciteit, gezinsinkomen, opleidingsniveau, slaaptekort, roken, tevredenheid met lichaamsgewicht voor de zwangerschap, de inschatting van de eigen BMI, het hebben van een gewichtsdoel en het krijgen van een GTZ advies van de verloskundige. Van de onderzochte vrouwen had $42 \%$ een GTZ conform de IOM-richtlijnen, $14 \%$ had een te lage GTZ en 44\% een te hoge GTZ. De BMI en de voeding waren geen factoren die geassocieerd waren met een GTZ volgens de richtlijnen. De determinanten voorspelden maar een klein deel van de verschillen die er zijn tussen vrouwen uit de drie groepen. Een GTZ boven ten opzichte van conform de richtlijnen was geassocieerd met een vermindering van de beweging (OR 0.54, 95\% $\mathrm{Cl} 0.33-0.89)$. GTZ onder, ten opzichte van conform de richtlijnen, werd vaker gezien bij vrouwen die een hogere score gaven op slaaptekort (OR $1.20,95 \% \mathrm{Cl} 1.02-1.41$ ), bij Kaukasische vrouwen in vergelijking met niet-Kaukasische vrouwen (OR 0.22, 95\% $\mathrm{Cl}$ 0.08-0.56) en bij vrouwen die bleven doorroken tijdens de zwangerschap ten opzichte van vrouwen die niet rookten (OR 0.49, 95\% $\mathrm{Cl}$ 0.25-0.95). Minder beweging bleek de enige beïnvloedbare factor te zijn die geassocieerd was met een te hoge GTZ. Het voorkomen van de vermindering van bewegen lijkt daarom een mogelijkheid te zijn om vrouwen een gezondere GTZ te laten krijgen. Verder kwam uit dit onderzoek naar voren dat een interventie ter bevordering van een gezonde GTZ bij gezonde zwangere vrouwen gericht zou moeten zijn op alle zwangere vrouwen, onafhankelijk van hun BMI voorafgaand aan de zwangerschap.

\section{Hoofdstuk 4}

Hoe en waarom veranderen vrouwen tijdens de zwangerschap hun beweeggedrag?

Omdat we tijdens de studie, beschreven in hoofdstuk 3 , gevonden hadden dat een verminderde beweging was geassocieerd met een te hoge GTZ, verrichtten we een secundaire analyse van de data die verkregen waren met de studie die in hoofdstuk 3 is beschreven. We onderzochten de verandering in vijf activiteiten: wandelen, fietsen, sporten, snelle acties en werken in huis en tuin. We gebruikten wederom het ASE-model om de mogelijke determinanten met het gedrag in relatie te brengen.

Van de 455 vrouwen rapporteerden meer dan de helft een vermindering van beweging. Een vermindering van beweging was het grootst bij sport en bij snelle acties, maar ook de andere beweegvormen werden duidelijk verminderd. Een vermindering van beweging werd vaker gezien bij vrouwen die zichzelf voorafgaand aan de zwangerschap actief vonden, bij 
vrouwen die zwangerschap gerelateerde klachten ervaarden, bij vrouwen die het advies hadden gekregen om hun beweegpatroon aan te passen en bij vrouwen die al eerder zwanger waren geweest. Minder dan 5\% van de vrouwen ging meer bewegen. De motivatie om gezond te bewegen was positief geassocieerd met een positieve attitude over bewegen in de zwangerschap.

De bevindingen van dit onderzoek wijzen in eerste instantie op de invloed van bewegen op GTZ en op een belangrijke rol voor de verloskundigen: zij kunnen vrouwen voorlichten over gezond bewegen, hen helpen om bij het ervaren van zwangerschap gerelateerde klachten toch te blijven bewegen en hen mogelijkheden aanreiken hoe de vrouw veilig kan bewegen. Daarbij is het van belang om de informatie te herhalen voor vrouwen die al eerder een kind hebben gekregen.

\section{Hoofdstuk 5}

Gedrag en determinanten van dat gedrag van Nederlandse verloskundigen in relatie tot gezonde gewichtstoename tijdens de zwangerschap, fase 1: Kwalitatief onderzoek Om met de interventie goed te kunnen aansluiten bij het actuele gedrag van verloskundigen, werd in deze studie de gedragingen van Nederlandse verloskundigen met betrekking tot gezonde GTZ onderzocht.

Het ASE-model werd gebruikt om de semigestructureerde interviews met zes eerste lijns verloskundigen te houden. De onderzochte verloskundigen gaven aan dat ze op drie gebieden bezig waren met het beïnvloeden van de GTZ: GTZ monitoring (wegen en bespreken van GTZ), voorlichting over voeding en (in mindere mate) voorlichting over beweging. De determinanten van het ASE-model werden bevestigd en andere determinanten, zoals de visie die verloskundigen hadden over gezondheidsbevordering en hun rol daarin werden toegevoegd. Daardoor werd het ASE-model uitgebreid om het verloskundige gedrag in relatie tot GTZ te kunnen verklaren. Het aangepaste model werd gebruikt voor de ontwikkeling van een vragenlijst voor het volgende kwantitatieve onderzoek.

\section{Hoofdstuk 6}

Gedrag en determinanten van dat gedrag van Nederlandse verloskundigen in relatie tot gezonde gewichtstoename tijdens de zwangerschap, fase 2: Kwantitatief onderzoek Voortgaand op het model dat in de vorige studie ontwikkeld werd, werd in deze studie een vragenlijst ontwikkeld om het gedrag van verloskundigen in relatie tot GTZ op een kwantitatieve manier te meten. De vragenlijst werd ingevuld door 112 verloskundigen die in de eerste lijn werkten.

De ondervraagde verloskundigen waren redelijk actief in het monitoren van GTZ, voorlichten over voeding en minder actief in het geven van voorlichting over beweging. Regressie analyses van de drie gedragingen (monitoren GTZ, voorlichten over voeding en voorlichten over beweging) lieten zien dat deze gedragingen gecorreleerd waren met 
meerdere determinanten, waarbij vooral de attitudes, sociale invloed, eigen effectiviteit ten aanzien van de gedragingen, samenwerking met anderen, zichzelf zien als gezondheidsbevorderaar en barrières belangrijke determinanten bleken te zijn. De studie gaf daarmee inzicht in de determinanten die het gedrag van verloskundigen kunnen beïnvloeden. Deze inzichten werden gebruikt bij de ontwikkeling van de interventie (hoofdstuk 7).

\section{Hoofdstuk 7}

"Kom aan!" Ontwikkeling van een interventie, met behulp van Intervention Mapping, gericht op het bereiken van een gezonde gewichtstoename tijdens de zwangerschap. Hoofdstuk zeven geeft een beschrijving hoe de interventie "Kom aan!" is ontwikkeld. Daarvoor zijn de gegevens die verzameld zijn in de vorige studies gebruikt en is gewerkt via de methode van IM volgens Bartholomew et al.. Een Consortium van gebruikers en gerelateerde professionals begeleidde ons bij de ontwikkeling van de interventie, zodat deze zo goed mogelijk zou aansluiten bij de praktijk van alledag.

Als resultaat van de probleemanalyse (stap 1 ) werden twee programmadoelen geformuleerd (stap 2): 1) Zwangere vrouwen zorgen ervoor dat hun gewichtstoename binnen de IOM-richtlijnen valt en 2) Verloskundigen geven zwangere vrouwen adequate begeleiding zodat zij een GTZ hebben die binnen de IOM-richtlijnen valt. Om deze grote doelen te bereiken werden kleinere programma doelen geformuleerd. Deze werden verbonden met de determinanten die van invloed zijn op de veranderdoelen. Zodoende ontstond een matrix van kleine veranderdoelen, aan de hand waarvan passende theoretische modellen werden gekozen (stap 3). De gebruikte theoretische modellen waren het Trans Theoretische model (TTM), de Sociaal Cognitieve Theorie (SGT), de Goal Setting Theory en de Elaboration Likelihood Theory. De praktische methodieken die bij deze modellen horen werden gebruikt om de interventie onderdelen te bouwen (stap 4). De interventie kreeg als titel "Kom aan!" verwijzend naar GTZ en tegelijkertijd verwijzend naar de aansporing om waakzaam te zijn; oftewel: wees waakzaam om gezond aan te komen. De interventie bestond uit een geïndividualiseerd internet programma, een gesprek tussen de zwangere vrouw en de verloskundige, een scholing voor verloskundigen (stap 5) en een praktijkkaart voor verloskundigen. Het internet programma bestond uit a) een "digitale intake met voorlichting", waarbij vrouwen antwoord gaven op vragen en passende (op maat) informatie kregen over GTZ, voeding en beweging en b) een kortdurend terugkerend gedeelte, waarbij vrouwen een grafiek te zien kregen met de GTZ-curve die voor hun BMI werd aanbevolen en met hun eigen GTZ daarin afgezet. Op die manier kon de vrouw in een oogopslag zien of de GTZ van dat moment binnen, boven of onder de richtlijnen viel. Daarnaast waren er voorlichtingsfilmpjes te zien waarin zwangere vrouwen als rolmodel fungeerden. Zij vertelden over het belang van een gezonde GTZ en over de manier waarop zij gezonde beweging en voeding realiseerden. 
Er werden vragen ontwikkeld voor deelnemende verloskundigen en zwangere vrouwen om de interventie op procesniveau te evalueren (stap 6). Tevens werd een onderzoek gestart waarin het effect van de interventie werd bestudeerd. Dit onderzoek is in hoofdstuk 8 beschreven.

\section{Hoofdstuk 8}

\section{"Kom aan!" Evaluatiestudie van een interventie gericht op gezonde gewichtstoename tijdens de zwangerschap.}

Het doel van de studie, beschreven in hoofdstuk acht, was om de ontwikkelde interventie ter bevordering van een gezonde GTZ "Kom aan!" te evalueren. Hiertoe verrichtten we een niet gerandomiseerde pre-post interventie studie. Een groep zwangere vrouwen die de interventie niet had gekregen $(n=144)$ werd vergeleken met een groep vrouwen die de interventie wel hadden gekregen ( $n=129)$. We gebruikten de vragenlijsten die we eerder hadden gebruikt tijdens de studie onder zwangere vrouwen (hoofdstuk 3 en 4). De vrouwen vulden deze vragenlijsten in aan het begin van hun zwangerschap en bij een zwangerschapsduur van ongeveer 36 weken. De GTZ werd berekend met dezelfde methode als tijdens het onderzoek onder zwangere vrouwen. Deelname aan het programma werd bijgehouden door het programma zelf en door registratie van de vragen die zwangere vrouwen tijdens hun deelname aan het programma per mail aan de onderzoeker stelden. We gebruikten multinomiale regressieanalyses om het effect van de interventie op de GTZ, voeding en beweging te testen en corrigeerden daarbij voor meerdere confounders.

Uit de analyse bleek dat de interventie geen effect had op de GTZ, voeding of beweging van zwangere vrouwen. Wel bleek dat vrouwen met een adequaat doel voor GTZ vaker een GTZ conform de richtlijnen hadden. Vrouwen met een te hoog doel bereikten vaker een te hoge GTZ en vrouwen met een te laag doel bereikten vaker een te lage GTZ. Vrouwen die aan de vis norm voldeden en vrouwen die hoog waren opgeleid (ten opzichte van gemiddeld opgeleide vrouwen) hadden minder vaak een te hoge GTZ. Vrouwen die fulltime werkten (ten opzichte van parttime werkenden) en vrouwen die aan het begin van de studie voldeden aan de fruitnorm hadden minder vaak een te lage GTZ.

Tijdens de interventieperiode bleek dat niet alle vrouwen de herhaalde uitnodigingen voor het programma ontvingen. Daarnaast gaven vrouwen aan dat ze de gebruiksvriendelijkheid van het internetprogramma suboptimaal vonden. Dit kan een mogelijke reden zijn van het ontbreken van effect. Een analyse van alle beschikbare gegevens voor de procesevaluatie is gepland.

Concluderend moet gesteld worden dat de interventie op dit moment niet succesvol is in de beïnvloeding van GTZ, voeding en/of beweging. Opvallend was dat ook in deze studie de BMI voorafgaand aan de zwangerschap geen factor bleek te zijn die geassocieerd was met GTZ. 


\section{Hoofdstuk 9}

\section{Beschouwing}

In de inleiding werd de vraag gesteld "Kunnen we een wetenschappelijk goed onderbouwd programma ontwikkelen om Nederlandse verloskundigen te helpen met het effectief bevorderen van een gezonde GTZ?" In onze zoektocht bleek dat er geen interventie beschikbaar was waarmee meer gezonde zwangere vrouwen een gezonde gewichtstoename konden bereiken. De zwangere vrouwen die wij zelf onderzochten lieten zien dat het merendeel van hen een te hoge of te lage GTZ hadden. De enige veranderbare factor die hiermee geassocieerd was, was het verminderen van beweging. Zwangere vrouwen die minder gingen bewegen bleken vaker zwangerschap gerelateerde klachten te hebben, vaker het advies te hebben gekregen om minder te bewegen, vaker zwanger te zijn van een tweede of volgend kind en vaker actief te zijn geweest voorafgaand aan de zwangerschap. De verloskundigen die we onderzochten bleken gemiddeld actief bezig te zijn met het monitoren van GTZ, het voorlichten over voeding en minder actief in het voorlichten over beweging. Er waren veel determinanten geassocieerd met de gedragingen van de verloskundigen. De opgedane kennis werd gebruikt bij de stapsgewijze ontwikkeling van de interventie "Kom aan!", die bestond uit een scholing voor verloskundigen, een internet gebaseerd programma voor zwangere vrouwen en een gesprek tussen de verloskundige en de zwangere vrouw. Het effect van de interventie werd geëvalueerd met een pre-post interventie studie. Daarbij kon geen interventie effect worden aangetoond op GTZ, de voeding of de beweging.

Dat GTZ een complex onderwerp is werd door dit proefschrift bevestigd. Ook al zagen we bij de vrouwen die aan de interventie hadden meegedaan niet vaker een gezonde GTZ dan bij de vrouwen die niet aan de interventie hadden meegedaan, toch geven we vanuit dit proefschrift vier aanbevelingen die ertoe kunnen bijdragen dat vrouwen vaker een gezonde GTZ bereiken. (1) Het stellen van een gezond streefgewicht bevordert het bereiken van dat doel. (2) Onze aandacht dient gericht te worden op alle vrouwen, ongeacht hun BMI voor de zwangerschap. Immers, bij gezonde zwangere vrouwen is het bereiken van een gezonde GTZ niet geassocieerd met de hoogte van de BMI die de vrouw had voordat ze zwanger was. (3) Het lijkt de moeite waard om ervoor te zorgen dat zwangere vrouw tijdens de zwangerschap blijft bewegen. (4) Onze aandacht dient ook gericht te worden op het vergroten van de verantwoordelijkheid van verloskundigen voor de totale gezondheid van de zwangere vrouw. Verloskundigen die deze verantwoordelijkheid voelen zijn actiever met het stimuleren van een gezonde GTZ.

Ondanks het feit dat we het veelbelovend Intervention Mapping (IM) protocol gebruikten om de interventie te ontwikkelen, bleek deze interventie niet effectief te zijn in het verhogen van het aantal vrouwen met een GTZ conform de IOM richtlijnen. We zoeken via een procesevaluatie naar factoren die deze ineffectiviteit verklaren. Vooruitlopend daarop bespreken we drie belangrijke keuzes die we gemaakt hebben tijdens dit project. 
We hebben ons gericht op de doelgroep van gezonde zwangere vrouwen en verloskundigen. Een doelgroep die voor ons als medewerkers van de Academie Verloskunde voor de hand ligt en die we niet betreuren. Toch zijn er argumenten die ervoor pleiten om de doelgroep uit te breiden.

Verder zijn er inmiddels meer factoren bekend die geassocieerd zijn of mogelijk verband houden met een GTZ binnen en buiten de richtlijnen. Te denken valt bijvoorbeeld aan de inname van hormoon verstorende stoffen die rijkelijk vertegenwoordigd zijn in de huidige samenleving, het optreden van psychische klachten tijdens de zwangerschap, de algemene beschikbaarheid van voeding en beweegprogramma's en andere maatschappelijke factoren. Dat betekent dat ook andere doelgroepen zoals beleidsmakers, scholen, werkgevers, supermarkten, aangesproken kunnen worden om een gezonde GTZ te bereiken.

Een derde keuze die we gemaakt hebben is dat we de definitie van een gezonde GTZ gehanteerd hebben die volgens de IOM gesteld werd. Het is de vraag of dat voor de groep van gezonde zwangere vrouwen een adequate definitie is. De auteurs van de richtlijn geven zelf ook aan dat de afkappunten niet goed onderbouwd zijn. Recente onderzoeken laten argumenten zien voor zowel hogere als lagere afkapwaarden. Omdat er nog geen goed onderbouwde afkappunten bestaan is het discutabel om deze te strikt te communiceren met zwangere vrouwen. De focus tijdens de zorg zou wellicht beter op de leefstijl dan op de GTZ mogen liggen.

Een vierde keuze was om de interventie te ontwikkelen met behulp van het IM protocol. IM is een iteratief proces, maar is vanwege pragmatische redenen in deze studie wel vrij rechtlijnig ingezet. Mocht er in de toekomst verder gewerkt worden aan een interventie voor GTZ, dan bevelen we aan om de complexiteit van GTZ ook op een meer complexe manier te benaderen. Ook het ASE-model was hiervoor waarschijnlijk te simpel.

Ondanks het feit dat de interventie geen gemeten effect heeft op de GTZ, de voeding of de beweging, is er toch een dagelijkse praktijk waarin gehandeld moet worden. Verloskundigen willen graag antwoord op de vraag wat op dit moment de beste zorg is rondom GTZ. Daarom werd met behulp van bestaande literatuur een advies geformuleerd. Dit advies beschrijft de ideale situatie en komt erop neer dat verloskundigen zich vergewissen dat zij beschikken over uitstekende communicatieve competenties (onder andere motiverende gespreksvoering en shared decision making). De zwangere vrouw wordt voorgelicht over wat we weten en niet weten over GTZ. De verloskundige vraagt haar welke GTZ de vrouw zelf in gedachten had en dient daar op gepaste wijze feedback te geven. De zwangere vrouw hoort van de verloskundige dat de kilo's tijdens de zwangerschap "beter blijven plakken" en dat een hoge GTZ het moeilijker maakt om na de zwangerschap weer op het oude gewicht terug te komen. De BMI van de gezonde zwangere vrouw is geen reden om beter (of minder goed) op de GTZ te letten. Verder krijgt de zwangere vrouw te horen (lezen/zien) wat de voordelen zijn van bewegen tijdens de zwangerschap en dient ze zo nodig ondersteund te worden om eventuele zwangerschap gerelateerde klachten geen belemmering te laten zijn om voldoende te bewegen. Om dit te kunnen doen heeft de verloskundige tijd nodig. In 
onze interventie hebben we geprobeerd om via een online interventie deel te voorkómen dat de verloskundigen meer tijd kwijt zouden zijn met het stimuleren van een gezonde GTZ. Ook wij hebben ons bij het ontwikkelen van de interventie meer laten leiden door de roep van verloskundigen dat ze geen tijd hebben dan de roep van de zwangere vrouwen dat ze meer individuele coaching en begeleiding nodig hebben.

In dit proefschrift werd de ontwikkeling en de evaluatie van een interventie gericht op een gezonde GTZ beschreven. Het oogsten van de bijbehorende successen en missers is een volgende stap om vrouwen te ondersteunen bij het krijgen (of handhaven) van een gezonde leefstijl, inclusief een gezonde GTZ. Vrouwen, hun kinderen, verloskundigen en de samenleving hebben een dergelijke interventie nodig om de balans van een gezonde GTZ terug te vinden. 


\section{Valorisation}

In this valorisation addendum we explain the societal value of this thesis, "In search of balance: promoting healthy gestational weight gain". In addition, we describe how the findings can be used to benefit maternity care.

\section{Relevance}

The research presented in this thesis addresses gestational weight gain (GWG) in healthy pregnant women.

Unhealthy GWG is associated with obesity and overweight in both mothers and babies. Obesity is associated with health problems in life, which makes unhealthy GWG a social and economic burden for society. Therefore it is crucial to find ways to prevent unhealthy GWG. In this thesis, we add to the body of knowledge on how to stimulate healthy GWG in all pregnant women. As in 2015 in the Netherlands more than 150.000 children were born, and less than half of their mothers reached a healthy GWG, many women and their children are at risk for overweight and obesity related to their GWG during pregnancy.

\section{Pre-pregnancy Body Mass Index (BMI)}

To begin with, we revealed in two studies, both described in this thesis, that pre-pregnancy BMI in healthy women was not associated with GWG as recommended by Institute of Medicine (IOM)guidelines, which are the most common guidelines on GWG. These guidelines recommend for women of normal weight a GWG in the range of 11.5 to $16.0 \mathrm{~kg}$. Women with a lower pre-pregnancy BMI are advised to gain more weight for GWG within the IOM-guidelines than women of normal weight, and women with a higher pre-pregnancy BMI are advised to gain less weight for GWG within the IOMguidelines. In the international literature pre-pregnancy BMI is recognized as a factor associated with GWG outside the IOM-guidelines. This means that overweight and obese women, when compared to normal weight women, more often have higher GWG than the guidelines recommend. Pathology also tends to occur more often in these categories. In contrast to the international literature, our studies involved samples of healthy pregnant women from all pre-pregnancy BMI categories, and we found that pre-pregnancy BMI as such was not significantly associated with GWG within the IOM-guidelines. This finding means that it is wise to continue to make efforts to prevent too high and too low GWG of women in all pre-pregnancy BMI groups. Or, from the opposite perspective: we need to promote healthy GWG for all women, including healthy women with a normal pre-pregnancy BMI.

\section{Physical activity in pregnancy}

Our studies revealed that more than half of pregnant women indicated that they reduced their physical activity (PA) when they became pregnant. We found that PA is related to healthy GWG. An increase in the level of PA could result in more women reaching a healthy GWG, and experience less overweight and obesity in the postnatal period. Our intervention did not result in the hypothesized outcomes, i.e., an increase in PA and an increase in the number of women gaining healthy gestational weight. Additional efforts need to be made to investigate effective strategies for increasing the level of PA during pregnancy. Midwives revealed in a study described in this thesis that they do not feel well-informed on this issue. As a result, many women hear from care providers and also from their partners and colleagues that they should rest and take it easy during pregnancy. Research indicates, however, that PA during pregnancy reduces discomfort in pregnancy and contributes to normal delivery. It even contributes to a healthy GWG. The new instruction should be: engage in healthy PA, even though, or even better, because you are pregnant.

The combination of physical complaints and PA can seem counterintuitive. Women in our study revealed that pregnancy-related issues were a barrier to maintaining their usual PA. It is not easy to 
tell a tired woman experiencing pain that she should continue her PA. It costs energy to promote PA in a woman with serious complaints. We can imagine that the social environment empathizes with a woman with complaints and it is understandable that partners, parents and peers are not in the position to motivate the woman to overcome her reluctance to engage in normal PA. Feeling sorry for someone is not the best attitude to help them through a difficult period in life, however. Prenatal care providers should be well-informed of the benefits of PA in pregnancy and should be able to motivate pregnant women to engage in healthy PA. Knowing that prenatal care providers can have a blind spot with respect to PA helps us realise that they need training so as to be informed and motivated to discuss this issue with the pregnant women in their care.

\section{The role of diet in healthy pregnant women in relation to their GWG}

In our review of interventions we found that dietary prescriptions for obese women, including support to stick with a diet, helped them to lower their GWG by more than eight kilograms on average. In our studies with samples of healthy women, we did not detect an association between GWG and diet. However, we only measured fruit- and fish-norms and the gram weight of vegetable consumption. The mean vegetable consumption was lower than recommended; less than half of healthy women ate enough fruit according to the norm, and only a small percentage of pregnant women ate the recommended amount of fish. Since vegetables, fruits and fish do not make up a complete diet, we cannot conclude that diet altogether is not associated with GWG. Like other researchers, we suggest that long-term, validated dietary studies of pregnant women are necessary to be able to draw conclusions regarding diet, GWG and the health of pregnant women and their offspring. Informal information exchange during the course of this study provided examples of how women's dietary preferences changed during pregnancy. Vegetarian women sometimes craved meat and meateaters wanted to eat vegetarian, for example. Women wished to eat more or less sugar, or more or less fat than they did while they were not pregnant. In a study described in this thesis, we found that diet was a difficult subject for prenatal care providers. They did not view themselves as having expertise about healthy food and lacked confidence in their dietary knowledge. Women when not pregnant have to sort out themselves what diet best suits them, no easy task considering the variety of opportunities of the current diet. In order to help pregnant women sort out what is best for them in their situation and for their body, it would be wise to offer every pregnant woman at least one hour of consultation with a dietician with expertise in pregnancy nutrition. Additional consultation may be necessary for women who experience difficulty in finding their dietary balance.

\section{Set a GWG goal, but what goal?}

Setting a goal for healthy GWG is associated with healthy GWG. Our study revealed that only $25 \%$ of women set a GWG goal and even fewer discussed a GWG goal with their midwife. Prenatal care providers should be convinced that women who set a healthy GWG goal more often reach that goal. In the discussion we reflected on our choice to use the IOM-guidelines to establish a GWG goal and concluded that these guidelines lack the support of sound evidence.

Given this lack of evidence for the GWG guidelines, we could consider that women may be capable and wise enough to set a healthy GWG goal for themselves. If prenatal care providers inform women about physiological GWG and the relationship between too high (and too low) GWG and problems in pregnancy and at the long term, pregnant women should be able to set a GWG goal that is appropriate to their situation.

\section{Where should the resources go?}

An interesting question in promoting healthy GWG has to do with the focus of our financial resources and efforts. Should we focus on pregnant women or should we invest in the prenatal care providers who care for pregnant women? Or in both? 
Given that prenatal care providers are seen as experts, their skills and knowledge should go further than the knowledge of an average pregnant woman. But as we have seen with respect to the issue of $\mathrm{PA}$, some prenatal care providers make recommendations counter to what is current knowledge (e.g., to slow down), which may be potentially harmful to pregnant women. We also noticed the restraint of participating midwives with respect to providing dietary advice. For this reason it is important that prenatal care providers stay up-to-date with current knowledge on issues concerning pregnant women. However, staying well-informed can be challenging in the busy practice of midwives. Some issues also require skills, in addition to knowledge, in order to be properly addressed. For instance, motivational interviewing can make the difference in motivating a pregnant woman to overcome barriers to engaging in healthy PA. During the development of "Come on!", we considered providing motivational interviewing training for midwives. Such a training did not fit in the narrow schedule of the program in the time available, however. In the future, providing motivational interviewing training should be reconsidered in GWG programs.

In conclusion, we think that financial resources should go to training and enhancing the competence of midwives, with the result that they can then help women better inform themselves and better evaluate the information they acquire. Money and efforts should also go toward providing reliable and easy-to-use information that explains current knowledge to all stakeholders, including pregnant women. For women who do not actively seek information, prenatal care providers should be proactive in providing information and raising awareness about the issues in pregnancy that affect the future health of the mother and her baby.

\section{Target groups}

This thesis is valuable for several target groups. Recommendations for these target groups are given below.

\section{Pregnant women}

Pregnant women can learn from this thesis that they need to be aware of their own GWG. They should consider setting a GWG goal, discuss this goal with their midwife and ask their midwife for support to attain this goal. Furthermore, pregnant women can learn that maintaining (or increasing) PA during pregnancy is well-advised for achieving a healthy GWG, but also in relation to the upcoming birth. In the follow-up from this thesis we produced a short educational film on PA in pregnancy for pregnant women. This film is available on the internet (https://www.youtube.com/watch?v=F-9mg_Cg2fO) and on the websites of a number of midwives.

We believe that the findings in this thesis confirm that it is important to hear the voices of pregnant women. We see reasons to stimulate pregnant women to make their own decisions, to set their own GWG goal, as long as they are aware of what is happening in their body. Pregnancy is a special life experience, and a sign of health, not a reason for being scared or overly dependent on others.

\section{Midwives}

Reflecting on our choice to develop an intervention in the form of a tailored internet program, rather than on in-person discussion with the midwife, we now believe that we have reasons to make a different choice. We advise midwives to spend more time on providing information to and raising awareness in pregnant women about changes in the body caused by pregnancy, including optimal GWG based on her pre-pregnancy BMI, changed dietary preferences and pregnancy-related barriers that may prevent women from being physically active. By doing so midwives can help pregnant women set appropriate GWG goals that match their personal weight attitude. Furthermore, when women face problems with maintaining PA, midwives can help them by using their knowledge and skills to promote health-enhancing behaviour. Midwives can also help pregnant women with their concerns about their diet and can refer them to a nutritionist for dietary consultation. If midwives have not been trained in engaging in health promotion conversations with pregnant women, they 
could be given skills training in motivational interviewing and shared decision-making. In addition, midwives can organize themselves and advocate for financial remuneration for advising pregnant women with respect to healthy lifestyle. Finally, midwives can collaborate with dieticians and physiotherapists so that they all disseminate the same messages regarding GWG, diet, PA and health in pregnancy. Using each other's knowledge and skills reinforces the messages given and the positive attitudes of the health workers. Schools for midwifery education can play an important role to increase midwives' involvement in health promotion.

\section{Physiotherapists and sport coaches}

Sport coaches and physiotherapists should encourage pregnant women to be active and to overcome barriers to engaging in physical activity. Preferably, they should collaborate with midwives and dieticians to provide the same advice.

\section{Dieticians}

Dieticians should organize their profession to advocate that pregnant women receive at least one hour of dietary consultation free of charge. They should provide pregnant women not only with information about what not to eat, but also with information about healthy (and easy) food choices. Dieticians should collaborate with midwives and physiotherapists and should reinforce the message to pregnant women that they continue to be physically active.

\section{Researchers}

Researchers should continue researching ways to promote healthy GWG, ways for improving PA during pregnancy and further investigate why the intervention "Come on!" did not provide measurable effects on GWG, diet or PA. Furthermore, we need evidence-based GWG guidelines for our population. Research in a population of healthy pregnant women could be a reasonable way to provide evidence-based margins for GWG.

\section{Policy makers}

Policy makers should provide financing for up-to-date, non-commercial information on healthy lifestyle for pregnant women. They should be aware that it is worthwhile to invest in the health of pregnant women and their babies. Pregnant women who request facilities (e.g., attending Mom in Balance $^{\circledast}$, membership in a sportclub) should be helped to get them. Prenatal care providers should be afforded the opportunity to educate themselves in shared decision-making and motivational interviewing. Ways should be found to provide this in efficient care models. Centering Pregnancy ${ }^{\circledast}$ could be a good option. Policy makers should work with health professionals, including midwives, dieticians, physiotherapists and general practitioners.

\section{Health insurance and municipalities}

Health insurance providers need to continue their efforts to help women stay healthy during pregnancy without patronising them. Pregnant women should be encouraged to choose a healthy lifestyle and to raise their babies in a healthy (prenatal) environment. Health insurance companies should talk with prenatal care providers to find out what their needs are. Prenatal care providers may need support, training and time to take up their health promotion role. With adequate financial investment, prenatal care providers can learn communication skills, and new forms of prenatal care, such as Centering Pregnancy ${ }^{\circledR}$, can be integrated. Collaboration with health workers is essential. When midwives advocate for pregnant women, they should be taken seriously. 


\section{Activities and products}

This thesis contains studies conducted in the context of Dutch midwifery. Publications and oral presentations related to this thesis are listed below. It is necessary that the physiology of pregnancy and childbirth become more visible in the academic world. This project is also valuable because another midwife has been added to the ranks of midwives skilled in doing research.

\section{Publications}

- Merkx A, Ausems M, Budé L, de Vries R, Nieuwenhuijze MJ. Interventions aiming to achieve a healthy gestational weight gain: a systematic review/meta analysis. Posterpresentatie Kennispoort, 3 februari 2012 Utrecht

- Merkx A, Ausems M, Nieuwenhuijze M, Budé L, de Vries R. Gestational weight gain in the Netherlands: what do pregnant women eat and why do they do so? Maternal and Child Nutrition 2013, Supplement 3: 1-38

- Merkx A, Ausems M, Budé L, de Vries R, Nieuwenhuijze MJ. Wat doen verloskundigen voor zwangere vrouwen om een gezonde gewichtstoename te bereiken? Poster, Nederlands Congres VolksGeZondheid, 4 april 2013 Ede.

- $\quad$ Ausems M, Merkx A, Budé L, de Vries R, Nieuwenhuijze MJ. Predictors of gestational weight gain (GWG), poster, European Congress of Epidemiology - Healthy Living, 25 June 2015 Maastricht

- Merkx A, Ausems M, Budé L, de Vries R, Nieuwenhuijze MJ. Dutch Midwives' Behaviour and Determinants in Promoting Healthy Gestational Weight Gain, Phase 1: a Qualitative Approach. International Journal of Childbirth 2015, 5,3, 126-38.

- Merkx A, Ausems M, Budé L, de Vries R, Nieuwenhuijze MJ. Dutch Midwives' Behaviour and Determinants in Promoting Healthy Gestational Weight Gain, Phase 2: a Quantitative Approach. International Journal of Childbirth 2015, 5,3, 139-53.

- Merkx A, Ausems M, Budé L, de Vries R, Nieuwenhuijze MJ. Weight gain in healthy pregnant women in relation to pre-pregnancy BMI, diet and physical activity. Midwifery 2015, (31) 7, 693701.

- Merkx A, Ausems M, Budé L, de Vries R, Nieuwenhuijze MJ. Bewegen tijdens de zwangerschap! Posterpresentatie Kennispoort, 22 januari 2016 Utrecht.

- Merkx A, Ausems M, de Vries R, Nieuwenhuijze MJ. “Come On!” Using Intervention Mapping to help healthy pregnant women achieve healthy weight gain. Public Health Nutrition, 2017;1-15 doi:10.1017/S1368980017000271

\section{Oral presentations}

- Gewoon Gezond Zwanger: interventies ter bevordering van een normale gewichtstoename. 25 oktober 2011, Symposium De zwangere centraal, naar multidisciplinaire samenwerking rondom geboortezorg in de Regio Rivierenland, Tiel.

- Gewichtstoename in de zwangerschap: een review en meta-analyse van interventies, 11 april 2012, Nederlands Congres VolksGeZondheid Amsterdam.

- Gestational weight gain in the Netherlands: what do pregnant women eat and why do they do so? 11 juni 2013, Nutrition \& Nurture in Infancy \& Childhood conference, Grange over Sands.

- Gewichtstoename in de zwangerschap: een review en meta-analyse van interventies, 29 november 2013, Kennis in Bedrijf, Hogeschool Zuyd Heerlen.

- Workshop How to teach (student) midwives to work with parents, 29+30 nov 2013, EMA Congress Maastricht.

- Midwife-led care and public health: Experiences and preferences about multidisciplinary collaboration. June 2014. International Confederation of Midwives Triennial Congress, Prague.

- Public health in midwife-led care: Opportunities, challenges and dilemma's, Healthy Gestational Weight Gain. June 2014. International Confederation of Midwives Triennial Congress, Prague. 
- Programma ontwikkeling gewichtsbegeleiding bij zwangeren (Intervention Mapping): 12 februari 2015 Interactief Avondseminarie Zwangere vrouwen begeleiden naar een gezonde leefstijl. UC Leuven.

- Het bevorderen van de gezondheid van zwangere vrouwen: de rol van bewegen. Symposium ter gelegenheid van de inauguratie van Marianne Nieuwenhuijze als lector aan de AVM-Zuyd. mei 2015, Maastricht.

- Bewegen tijdens de zwangerschap, Wetenschappelijke avond voor sportgeneeskunde, 4 april 2017, Bilthoven.

\section{Education}

- Teaching midwives and practice assistants how to work with the program "Come on!", April 2010.

- Bewegen tijdens de zwangerschap. Contribution to the Massive Open Online Course (MOOC) of the Academie verloskunde Maastricht, Maastricht 2015

- Several activities for student midwives to teach them how to work with different aspects of developing an intervention (using Intervention Mapping, Tailorbuilding, Videoscribe, designing multifaceted interventions).

\section{Products}

- Educational film for midwives to provide them with scientific information on PA and pregnancy. The aim of the film is to increase the positive attitude toward stimulating pregnant women to keep on moving during their pregnancy. https://www.youtube.com/watch?v=5aZiToCNzTw\&t=11s

- Educational film for pregnant women to stimulate them to stay physically active. https://www.youtube.com/watch?v=F-9mg_Cg2f0

- Midwifery card to provide midwives with IOM-guidelines in a visual easy-to-read manner.

\section{Innovation}

Since an effect of the intervention "Come on!" on GWG, diet or physical activity, was not proven, the program is not ready to implement. However, the change objectives formulated in this thesis are underpinned with research, which make them valuable for reuse by other researchers and program developers.

The animation film about PA for pregnant women is finding its way to the websites of midwives. The thesis, presented at the Dutch forum of midwives (Kennispoort), and will make its way through the professional organization of midwives. 


\title{
Dankwoord
}

\author{
Continuous effort \\ - not strength or intelligence -
}

is the key to unlocking and using our potential.

\section{Liane Cordes}

Dank aan mijn ouders. Het leven is een onbeschrijfelijk geschenk. Als mijn moedert in deze tijd zou zijn geboren, was ze misschien wel verloskundige geworden, maar had ze dan zelf ook nog negen kinderen gebaard? Ik ben blij dat ik als negende toch geboren ben. Als mijn vader in deze tijd zou zijn geboren, had hij kansen gekregen om te leren en was hij misschien professor geworden.

Waarschijnlijk op het gebied van landbouw of zelfvoorziening. Ik vind hem een onderzoeker in hart en nieren, maar het leven leidde hem naar andere wegen. Mijn ouders hebben mij en mijn broers en zussen gestimuleerd om te studeren en om onze capaciteiten ten volle in te zetten in de wereld. Mijn ouders hebben mij ook geleerd om door te zetten; vooral door hun voorbeeld, waarbij ze in financiële armoede een groot gezin van alle behoeftes wilden voorzien. Ik heb gedurende de jaren steeds meer waardering voor mijn ouders gekregen, die door "continuous effort" voorgedaan hebben hoe je je potentieel kunt ontgrendelen.

Naast mijn vader zijn er nog andere belangrijke mannen (ja, mannen een keertje eerst) die mij geholpen hebben bij het verwezenlijken van dit proefschrift en die ik bij deze heel hartelijk wil danken. Raymond de Vries, die mij als professor de gelegenheid gaf onder zijn vleugels te promoveren. We hadden niet zo heel vaak contact, maar als we contact hadden was het inspirerend. Frans Merkx, mijn lieve broer, van het begin tot het einde was je betrokken en ik vermoed dat je (samen met Irene) meer dan eens in Lennisheuvel toelichting hebt gegeven op wat ik aan het doen was. Je kritische kanttekeningen en je waardering voor mijn ijver hebben me vooruit geholpen. Je steunde mij door dik en dun en noemde me met regelmaat je "dappere zus", iets wat ik niet altijd zelf kon voelen, maar wat me wel steeds weer ontroerde en verder hielp. Peter Jeurissen, door je respect voor mij en voor de wetenschap stimuleerde je mij om mij Master opleiding te volgen en om de uitdaging van een promotiebaan aan te gaan. Bert Zeegers, jij hebt onbedoeld en onvrijwillig, maar toch, ruimte gemaakt, zodat ik in jouw plaats deze promotieplek kon innemen. Ik kon niet anders dan mijn best doen. En dan maar steeds zeggen dat ik jou inspireer, terwijl het andersom net zo is. Thijs Lenders, op het moment van erop of eronder was jij er die (eindelijk) mijn stukken doornam en verwoordde waar ik geen woorden voor kon vinden. Je hebt mij niet anders gekend dan "bezig met mijn promotie", terwijl ik je gunde dat je mij vrij van dat had kunnen zien. Luc Budé, jouw eerlijke en oordeelvrije feedback was verhelderend. Soms had ik het idee dat jij de enige was die begreep, dat wat ík bedoelde ook zo stom nog niet was. Kees en Giel van Koppen, zonder wie ik niet had beleefd wat moeder zijn is. Ik noem jullie samen, maar jullie hebben ieder apart en ieder op jullie eigen manier mij gesteund in mijn weg. Door me keer op keer te helpen aarden, door te verlangen dat ik er ook voor jullie was en door me ruimte te geven. Ik ben enorm trots op jullie. En Benjamin Smit, die er ineens was, een tijdje bleef, mij de ruimte liet, mij aanvulde en die en mij vleugels gaf om dit traject op een mooie manier af te ronden. Dank met een diepe buiging aan jullie heren!

Er waren nog veel meer mensen die me ondersteunden bij de verwezenlijking van dit traject. Uiteraard Marlein, bedankt, mijn eerste aanspreekpunt en dagelijkse begeleider. Altijd 
goedbedoelend, maar jeetje, wat hebben we een kluif gehad aan het begrijpen van elkaar. Mijn tranen en jouw (door mij zo ervaren) wazigheid in het begin waren de tegenpool van de goede moed en de duidelijkheid die er later voor in de plaats kwamen. Dank dat je in mij bleef geloven. Marianne, de eerste tijd zelf zo druk, maar op het goede moment weer daar om bij te sturen, mij te ondersteunen en te confronteren. Je hebt nu gezien waartoe ik in staat ben. Esther Bakker, je was de pionier; de eerste doctor in de Vroedvrouwenschool en daarmee een eye-opener voor mij, en Marjan Govaerts die ik zag onderzoeken en op weg was naar haar titel. Darie, Evelien, Hennie, Irene, Isabel, Marijke, Pien, Tamar, Tjarda als mede onderzoekers, maatjes, altijd bereid om vragen te beantwoorden, om zorgen te delen, om me te bemoedigen. Yvonne, door jouw andere manier van benaderen was je een voorbeeld. Door jouw opstelling heb ik geen twee promotietrajecten doorlopen, maar kon ik me focussen op mijn eigen project. Rachelle van der Wagt, Cecile van Lümig, Sanne Kohl, Ellen Kant, Anita Badart, Anita Stevens, Anke van der Vorst, Esther Schoffelen, Mayke van Ansenwoude, Meno Tomatala; jullie zijn (een stuk) met me meegelopen, hebben meegedacht en hebben me bevestigd in wat ik aan het doen was. Mieke Clement, je bent een vroedvrouw in hart en nieren, je ging me voor met je Masters en je geloofde in mij. Ook dank aan de verloskundige doctoren die me voorgingen en mij inspireerden. Dank aan Marja, Nardie, Rafael en Raymond die mijn directeuren waren in de tijd als docent en junior onderzoeker.

Dank aan de collegae die mij zagen verdwijnen, worstelen en weer zagen bovenkomen; collegae die wilden weten hoe het met mij als mens ging. Han (en Leon) natuurlijk, en Meta, mijn maatjes van alledag (en dus van al mijn ins en outs) en verder in alfabetische volgorde: Agnes, Angelique, Anne, Anneke en Anny, Anja, Brenda, Carin, Christianne, Daan, Daniëla, Dineke, Ed, Els en Els, Elske, Emer, Florien, Frans, Gabrielle, Gemy, Geneviëve, Hannie, Helma, Ina, Inge, Ingrid, Irene, Janneke, Janet, Jeanne, Jeannette, Joke, Joyce, Josette, Josien, Judith en Judith, Karin, Karen, Kiki, Korien, Latifa, Leonie, Linda, Lisette, Lizette, Lotte, Maaike, Marja, Marjan, Marjolein, Maureen, Meike en Meike, Mieke, Miep, Miriam, Nathalie, Patrick, Peter, Rachèl, Renée, Reny, Rica, Robbie, Sabrina en Sabrina, Simone, Susanne en Suzanne, Tonnie, Vivian, Willeke, en Xandra. Ook dank aan Michaela Schuldt, en de andere mede-master studenten. Dank aan de studenten en alumni van de AVM, die mij zo rijkelijk voorzagen van een mengsel van leergierigheid, dankbaarheid, interesse in mijn proces en vriendelijkheid. Ik ben trots op onze toekomstige verloskundigen met zo'n hartelijke en persoonlijke betrokkenheid. Ik mocht op jullie oefenen met journal clubs, ik mocht jullie enthousiast maken voor onderzoek en geregeld hingen jullie aan mijn lippen. Ik weet dat ik een "rare docent" ben, een vreemd eend in de bijt, en ik haal jullie regelmatig uit jullie comfortzone. Jullie waardeerden mij (toch) in een heftige periode van mijn leven. Wat is er leuker?

Dank aan alle zwangere vrouwen die hun gegevens invulden en meedachten met uitprobeersels van vragenlijsten en interventie onderdelen. Verder dank natuurlijk aan alle verloskundigen die mee werkten aan de onderzoeken, en dank aan jullie assistenten die mij regelmatig te woord stonden. Zonder "de krakelingen" zou het ondenkbaar zijn geweest. Bedankt Jeanne, Annemieke, Jacqueline, Tiny, Jozien en Anja en de bijbehorende "lange vingers" Jos, Louis, Marius, Frans, Tinus. Jullie niet aflatende geloof in mij en het delen van de gebeurtenissen in jullie levens gaf en geeft mij een enorme verbondenheid. Ik weet dat jullie er zijn en dat ik weet dat ik meetel voor jullie. Ook de andere mensen die ik ken vanuit Eckelrade, waar ik woonde toen ik begon. Het lijkt zo lang geleden. Inmiddels ben ik twee keer verhuisd, maar het was in Eckelrade dat "de tijd daar was"; dat de inspiratie, het vertrouwen en het lef om te beginnen bij elkaar verzameld was. Pauline Jeurissen, dank voor de openheid en de mogelijkheid om weer een stap te zetten. 
Kees, Willy, Nelly en Sjef, Rinit en Marjo, Sjef, Frans en Irene, Marja en Ellen, Ton en Odette; jullie horen bij mij, ik vertrouw jullie en ik ga er graag van uit dat jullie weten dat ik mijn steentje bij draag om het in de wereld een ietsje meer kloppend te maken. En al weet ik niet precies hoe het zit, deze promotie is ook opgedragen aan jullie.

Imara en Yolande, twee prachtvrouwen; een speciaal woord aan jullie; omdat jullie mijn zonen gelukkig maken. Ik weet niet of je het begrijpt, maar door hun keuze voor jullie word ik bevestigd in mijn moederschap. Ik heb er twee schone dochters bij gekregen. Ook dank aan Arthur, Carlaine, Jitse, Xander, en Dinand; jullie hebben mij ieder op jullie eigen wijze bevestigd in mijn weg. Ik had geen enkel recht, maar jullie lieten me toe als bonus-moeder.

Arend, Diana, Koos en Jannie, Corry, Gordana, Brenda, Michelle, Kitty en Hans, heeroom Tinus ${ }^{\dagger}$, jullie lieten me met empathie in de spiegel kijken en hielpen me daardoor met het recht strijken van de onvermijdelijke kreukels van mijn geschiedenis. Annet (heerlijk dat je kookte!), Paul, Steffi, Frederique en Josephien, Nicole, Ton van la Source, Jo Heusschen, Peggy, Frank Corvers, de Pippen, Yvonne Velthuis, Paul Alblas en de anderen van het CNME, Aline, Frits, Jack, Jarno, Wilma, Fredt, Yoka, Ed, Fleur en Merel, Michel, Hans, Martijn, Lisette en Erik, de andere twee schaapsherders, Edo en Brigitte, Wouter van Eck, Pascal en Jean-Pierre (dank voor de computer!), Trudy, Marinka, Annemarie, Marjo, Carla, Florian, Frank, Peter en Thea Bodelier, Barbara, Rolf, Ploni, Sanne, Annemieke en Ed, Maryvon, Mike, Paul, Ellen, André Kaelen, Servaas, Jill, Martin, Anna-berth, Bob, Simon, Till, Hans, René en Lisette, Ann. Alle vrienden en bekenden uit Lemiers, Holset, Vaals die me zo'n heerlijk thuis hebben gegeven. De mensen van VELT, de mensen van de permacultuurcursus en de voedselbos club. De mensen van de gemeente Vaals, de vrienden op de fiets, de vele vrijwilligers. Jullie warmte en liefde verwarmen mijn hart. In de dagelijkse dingen schuilt zoveel verbinding. Ik ken zoveel mensen en ervaar zoveel steun dat ik vast nog iemand vergeten ben die het niet verdient om in mijn lijst te ontbreken. Want het is ook de man die ik tegenkom op straat en die naar me lacht, de onbekende vrouw die vraagt of ze met me mee kan rijden. De man met sigaar en een hondje die zwaait, de portier die me groet en me fijn weekend toewenst, de vuilnismannen en mensen van de huishoudelijke dienst, die steeds weer zorgen dat het schoon en opgeruimd is, de servicedesks van ICT en bibliotheek, de printshops als er een poster geprint moest worden, de mensen van Mas con Menos en de deelnemers aan de workshop rocketstove bouwen. ledere keer opnieuw zoveel mensen die me laten merken dat we allemaal verbonden zijn. Door die wetenschap word ik gedragen. Dus ook aan alle niet genoemden heel veel dank.

Credits wil ik ook uitdelen aan de volgende bedrijven: Banditos, voor de heerlijke Latte Machhiato's. Endnote, voor de mogelijkheid om referenties op een redelijk eenvoudige manier te koppelen aan de geschreven teksten. Heuvellandcursus, die me ondersteunde in mijn Engels schrijfproces. N-vivo, voor de mogelijkheid om kwalitatieve data te analyseren (hoofdstuk 5). Microsoft, voor de programma's Word, Excell, Visio, Powerpoint. Questback, voor de mogelijkheid om via internet vragenlijsten af te nemen. REVman, voor het verrichten van een meta-analyse. SPSS, voor de programma's die ze leveren om statistische analyses te kunnen verrichten. Submedia, die me ondersteund heeft met het maken van de filmpjes en tekeningen voor "Kom aan!". Tailorbuilder, voor de mogelijkheid om het internet gedeelte van "Kom aan!" te maken. Videoscribe, waarmee ik filmpjes kon maken over bewegen voor zwangeren. Vimeo, voor de mogelijkheid om filmpjes toe te voegen aan "Kom aan!". Weleda, voor het doneren van verzorgingspakketjes voor een uitgeloot deel van de zwangere vrouwen die meededen aan de studie die beschreven is in hoofdstuk 3 en 4. 


\section{Curriculum Vitae}

Astrid Merkx werd op 11 september 1963 geboren in Boxtel als negende kind van Jan en Toos MerkxNijman. Na het behalen van het VWO diploma, begon ze haar professionele carrière met de opleiding tot A-verpleegkundige in de ziekenhuizen te Venlo-Tegelen en vervolgde met de opleiding tot Bverpleegkundige in Venray. Vervolgens studeerde ze in 1991 cum laude af als verloskundige aan de Vroedvrouwenschool te Heerlen. Na ruim zeven jaar als eerstelijns verloskundige in Eijsden en Margraten te hebben gewerkt, werd ze in 1999 als docent aangesteld aan de Vroedvrouwenschool in Kerkrade. Ze volgde de eerste graads leraren opleiding aan de Universiteit van Maastricht en in 2011 voltooide Astrid haar European Master of Science in Midwifery aan de Caledonian University in Glasgow. Ze onderzocht de huidige praktijk van Nederlandse verloskundigen met betrekking tot de preventie van kindermishandeling, waarbij ze het ouderschapsmodel van Alice van der Pas voor het eerst toepaste op de prenatale periode. In april 2011 begon Astrid aan haar promotietraject "Gewoon Gezond Zwanger".

\section{Overige publicaties}

- Merkx ACMJ. Soms gaat een baby dood, tijdens de zwangerschap, de bevalling of in de eerste tijd daarna. Eckelrade: Merkx; 1998.

- Govaerts MJB, Bisscheroux TJHI, Merkx ACMJ. Docentprofessionalisering door integratie van theoretisch leren, ervaringsleren, intervisie en reflectie. Tijdschrift voor Medisch Onderwijs 2004: 23(2)

- Merkx ACMJ, Heusschen J. Gespreksvaardigheden voor verloskundigen, een praktische handleiding. Utrecht: Lemma; 2004.

- Merkx A, Korstjens IM. Verloskundigen dragen bij aan primaire preventie van kindermishandeling door goed ouderschap te ondersteunen, een kwalitatieve studie. Tijdschrift voor verloskundigen. 2012, 37 